First Year Teacher

\title{
Internship Program
}

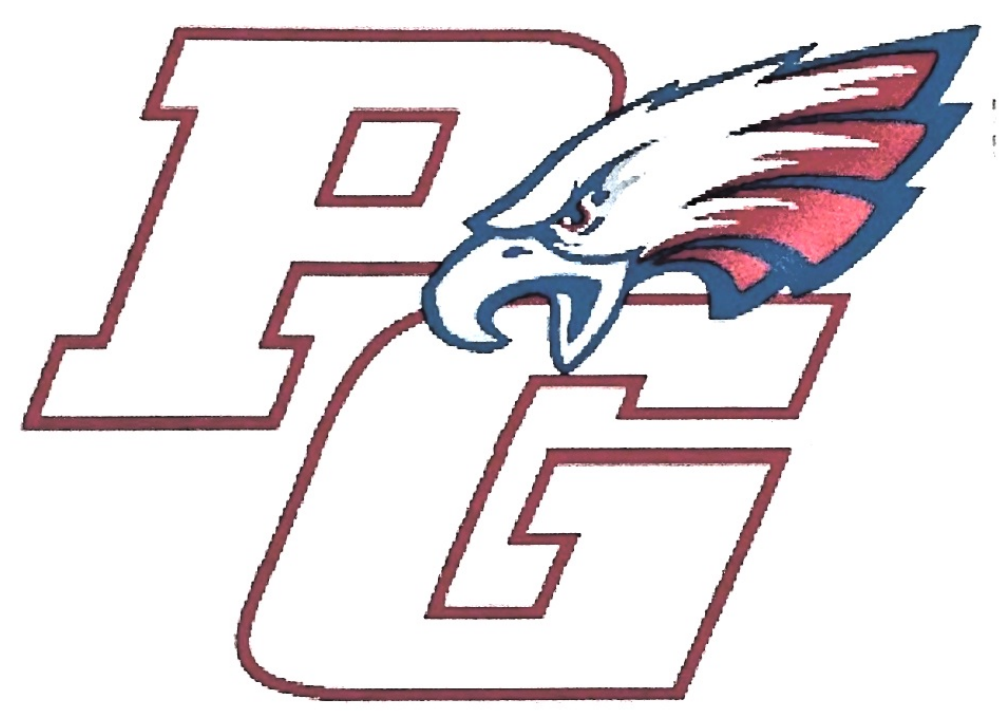

\section{Home of the Eagles}

\author{
Jessica Forster
}

Pleasant Grove High School Agriculture

Department

AGED 539, Cal Poly State University

Spring Quarter 2014 


\section{Table of Contents}

\section{Section 1: Reflection on Quality Criteria Standards}

Quality Critena 1: Cumculum and Instruction

Quality Criteria 2: Leadership and Citizenship Development

Quality Criteria 3: Practical Application of Agricultural Skills

Quality Criteria 4: Qualified and Professional Personnel

Quality Criteria 5: Facilities, Equipment, and Materials

Quality Criteria 6: Community, Business, and Industry Involvement

Quality Criteria 7: Career Guidance

Quality Criteria 8: Program Promotion

Quality Criteria 9: Program Accountability and Planning

Quality Criteria 10: Student-Teacher Ratio

Quality Criteria 11: Full Year Employment

\section{Section II: Support Materials}

Support Material 1: Student Data Sheets

Support Material 2: Permanent Student File

Support Material 3: Agriculture Course Outlines

Support Material 4: Course Gradebooks

Support Material 5: Completed SAE Supervision Forms

Support Material 6: SAE Project Statement in Syllabus

Support Material 7: FFA Statement in Syllabus

Support Material 8: FFA Program of Activities

Support Material 9: Recruitment Program

Support Material 10: FFA Chapter Scrapbook

Support Material 11: Summer Activities Calendar

Support Material 12: Graduate Follow Up Survey

Support Material 13: Graduate Follow Up Survey Results

Support Material 14: Comprehensive Program Plan

Support Material 15: Advisory Committee Meeting Agendas

Support Material 16: Advisory Committee Meeting Minutes

Support Material 17: Advisory Committee Constitution \& By-Laws

Support Material 18: Proficiency Standards

Support Material 19: Teaching Credentials

Support Material 20: Calendar of Activities

Support Material 21: Professional Growth \& Development

Support Material 22: R-2 Report 


\section{Table of Contents continued}

Support Material 23: Travel Request

Support Material 24: CATA Membership Card

Support Material 25: Report to Administration

Support Material 26: Five Year Acquistion List

Support Material 27: Operating Budget for Department

Support Material 28: Budget Process

Support Material 29: Chair Person's Duties \& Responsibilities

Support Material 30: Chart of Responsibilities

Support Material 31: Substitue Teaching Procedure \& Plans

Support Material 32: Description of Program Completer

Support Material 33: Articulation Agreement with Consumnes River College

Support Material 34: Reimbursement Process

\section{Section III: AGED 539 Project}

AGED 539 Project Description

AGED 539 Program of Activities 


\section{Section I}

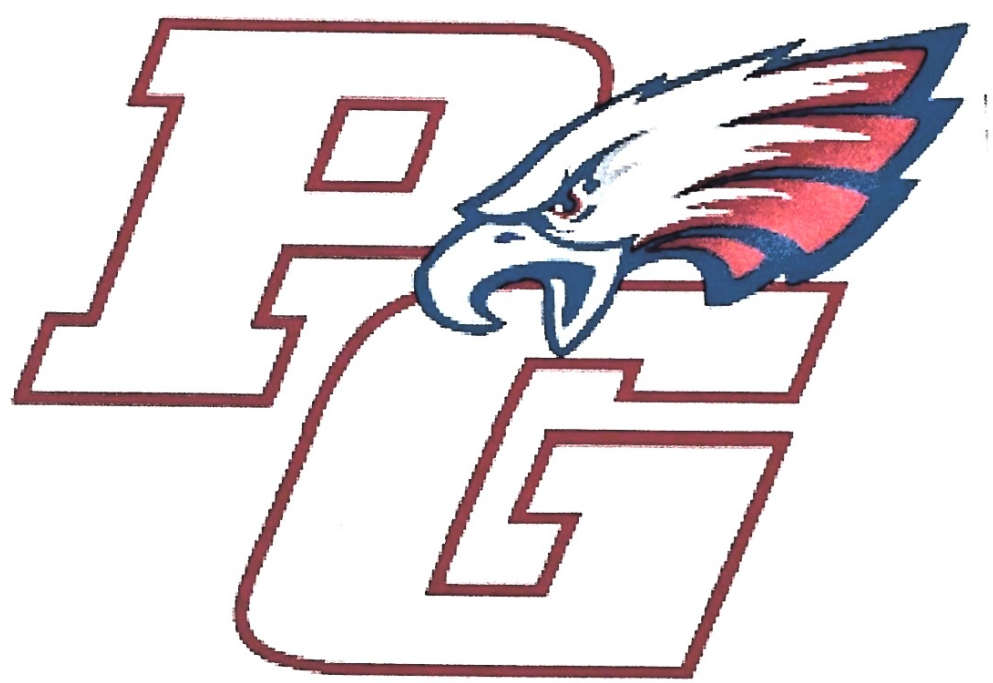

Home of the Eagles

\section{Refelction on Quality Criteria Standards}




\section{Table of Contents}

\section{Section I: Reflection on Quality Criteria Standards}

Quality Criteria 1: Curriculum and Instruction

Quality Criteria 2: Leadership and Citizenship Development

Quality Criteria 3: Practical Application of Agricultural Skills

Quality Criteria 4: Qualified and Professional Personnel

Quality Criteria 5: Facilities, Equipment, and Materials

Quality Criteria 6: Community, Business, and Industry Involvement

Quality Criteria 7: Career Guidance

Quality Criteria 8: Program Promotion

Quality Criteria 9: Program Accountability and Planning

Quality Criteria 10: Student-Teacher Ratio

Quality Criteria 11: Full Year Employment 


\section{Quality Criteria 1: Curriculum \& Instruction}




\title{
Pleasant Grove High School Agriculture Department
}

\section{Quality Criteria 1: Curriculum and Instruction}

Pleasant Grove High School (PGHS) Agiculture Department currently uses the California Career Technical Education Model Curricular Standards for Grades Seven through Twelve. These standards were adopted by the California State Board of Education in May of 2005. In addition to the CTE Model Curricular Standards, PGHS also utilizes the Science Content Standards for California Publich Schools Kindergarten through Twelve Grade, adopted by the California State Board of Education in October of 1998. These standards are utlized in conjunction with the Career Technical Model in our earth and physical sceince, horticulture, and animal science based classes (listed below), all of which are UC/CSU approved to meet the A-G rquirements as well as high school graduation requirements.

The curriculum used by the PGHS Agriculture Department is designed to allow students to follow one of two pathways - Agricultural Science or Ornamental Horticulture. Entering as Freshmen, students may choose Applied General Science (similar to an Agriculture Earth Science class) or Agriculture Biology, which is only available to students who were enrolled in an Honors Science class at the middle school level. Freshmen who were enrolled in Applied General Science continue as Sophomores with Agriculture Biology, and once Agriculture Biology is completed students may choose a Pathway. All courses in the PGHS Agriculture Department are UC/CSU approved and count towards graduation requirements, which is indicated on course enrollment forms provided to parents and students as well as the Agriculture Department's 4-Year Plan, which is provided to all first year students.

\author{
Agricultural Science Pathway: \\ Applied General Science \\ Agriculture Biology \\ Animal Anatomy \& Physiology \\ Veterinary Science and/or Economics in Agriculture
}

\section{Ornamental Horticulture Pathway: \\ Applied General Science \\ Agriculture Biology \\ Elements and Principles of Floral Design \\ Ornamental Horticulture and/or Economics in Agriculture}

Both pathways offer opportunities for a variety of teaching techniques and integration of technology. Each agriculture classroom is equipped with a Teacher Work Station, an integrated LCD Projector, VCR/DVD Player, and document camera; all run through a central control panel and surround sound speakers. There are 5 computer labs on campus, which can be reserved through a central school calendar. All students and teachers also have access to SchoolLoop, which allows for communication via "Loop Mail" as well as an internet based storage locker where eRecordBooks and iRecordBooks can be stored, as well as other materials for class. 
Quality Criteria 2:

Leadership \&

Citizenship

Development 


\section{Pleasant Grove High School Agriculture Department}

\section{Quality Criteria 2: Leadership and Citizenship Development}

Pleasant Grove High is one of several recently constructed schools in the Elk Grove Unified School District, being opened in 2005. The Elk Grove-Pleasant Grove FFA Chapter was chartered in 2005, and is supported by the students, teachers, adminstrators and parents of PGHS, while continuing to develop a prescent in the Elk Grove community.

Any student enrolled in an agriculture course at Pleasant Grove High School is automically a member of the Elk Grove-Pleasant Grove FFA Chapter. As an FFA member, students have the oppotunitity to develop premier leadership, personal growth, and career success through participating in various chapter, sectional, regional, state, a nd national activities. Through each course in the Agriculture Department, FFA is weighted at $10 \%$ of the students total grade, as well as a $10 \%$ weighted category for their Supervised Agriculture Experience project. The remaining $80 \%$ of student's grades are earned through other class work, projects and assessments.

To earn credit for their SAE project, students must maintain either an entrepreneurial or placement project and complete a minimum of 3 hours per month of work on the project outside of school. The project is then graded based on students completing their California Agriculture Education Record Book each month, with the grade received on their montly Record Book check being entered into the SAE category of the gradebook. The FFA portion of students grades is based on attendance at a minimum of two FFA activities per academic quarter. Attendance at activities is tracked through sign in/sign out sheets, which are kept on file throughout the year.

The majority of the students enrolled in PGHS agriculture courses will participate in more than the required two activities per quarter, and will encourage non-FFA members to paricipate in activities held on campus as well such as monthly FFA movie nights. This allows for a continual recruitment of new students throughout the year. While the majority of students are active FFA members, there are also some students who choose not to participate in FFA activites, and as a department we continue to encourage these students by providing a welcoming environment and having active members share their positive experiences. All FFA activities and meetings are organized by the chapter officer team; at each event, the designated FFA Advisor is present to oversee and supervise the activity.

Students who choose to raise a livestock project for the Sacramento County Fair are required to attend specie meetings throughout the winter and spring months where they receive information specific to their specie, fair infomration, etc. Students who maintain a year-round project or who exhibit at the CA State Fair are enrolled in the ROP Sales/ Service Class, which runs from May through July and allows students to earn elective credit. This class meets weekly to cover information for the fair, update project records, and participate in animal science lessons. 


\section{Quality Criteria 3:}

Practical Application of

(c) Agricultural Skills 


\section{Pleasant Grove High School Agriculture Department}

\section{Quality Criteria 3: Practical Application of Agricultural Skills}

At PGHS, our Agriculture Department is proud to offer students the opportunity to learn practical application of skills throughout each of our classes. During each course, students are able to participate in hands-on activities and labs which not only enhance their learning, but allow for the application of agricultural skills in a real and meaningful way. Some examples of these projects and experiences include: worming and vaccinting of animals at the school farm, feed trials, AgriScience Projects covering a multitude of agriculture based subjects, installing drip irrigation systems, updating and maintaining landscaping in and around the department, floral arrangements for large school district events and community members.

In accordance with the Agriculture Incentive Grant Criteria 3, each student enrolled in an agriculture course is required to have a SAE project, which is a part of the students grade for the course. The SAE poriton of student's grades counts as $10 \%$ of the total course grade, and is indicated in each Course Descripton and Syllabus that all parents and students receive and sign at the beginning of each year. Students must track their SAE hours and activities in the California Agriculture Education Record Book each month, with a minimum of 3 hours outside of normal class time being accumalted on the project. In each class, students have a designated Record Book Work Day each month, where they can update their Record Books in class and instructors can monitor and assist as needed. While some students in the department choose to simply take the zero on these monthly Record Book assignments, which equate to 90 points each month, usually within the couple months most students realize that it is a straight forward assignment which is not difficult to meet. Most of the students who hesistate tostart an SAE project, usually can find a small project that allows them to devleop practical, hands-on skills outside of class. Students within the department can apply for local, sectional,and regional Proficiency Awards, and can participate in local and sectional Project Competition.

PGHS is extremely fortunate to have an Agriculture Facility which was constructed over the course of the last two years. The facility includes two laboraty classrooms, an office area for department staff, and a "Project Room", which serves as central meeting place for chapter officers in addition to a large and small animal barn which is available for students to house fair projects including market steers, lambs, goats, hogs, poultry, and rabbits. Construction on a large free standing floral cooler was completed this past fall to allow Floral Design courses to expand, and next school year a greenhouse complete with an integrated teaching area will be open for students in Ornamental Horticulture classes. Additional, there is a small school orchard with 8 fruit trees of different varieities. There is also a Ford pickup and livestock trailer and scales to allow for easy monitoring of students SAE projects throughout the year. All gas expenses for the site's pickup are maintained through the District, and any personal mileage or expenses incurred throughout the year are fully reimbursed through the District with proper paperwork and documentation. 
Quality Criteria 4: Qualified \&

6

\section{Professional Personnel}




\section{Pleasant Grove High School Agriculture Department}

\section{Quality Criteria 4: Qualified and Professional Personnel}

All agriculture instructors at Pleasant Grove High School are in posession and maintain the appropriate credentialing for teaching the agriculture courses offered. For the 2013-2014 school year, all instructors attedn monthly staff meetings, in addition to department meetings and team collaobration session held on the PGHS campus.

\section{Izaskun Zallo}

Clear Specialist Instruction Credential: Agriculture

Clear Singel Subject Teaching Credential: Agriculture

Classes Assigned: Agriculture Biology, Animal Anatomy and Physiology,

Jessica Forster

Clear Specialist Instruction Credential: Agriculture

Clear Singel Subject Teaching Credential: Agriculture

Classes Assigned: Applied General Science, Elements and Principles of Floral Design,

Economics in Agriculture

Jenna Swenson

Clear Specialist Instruction Credential: Agriculture

Clear Singel Subject Teaching Credential: Agriculture

Classes Assigned: Applied General Science, Agriculture Biology

All agricultural staff attend the Sectional and Regional California Agriculture Teachers Assocition (CATA) meetings and Inservices, as well as the CATA Summer Conference held at Cal Poly, San Luis Obispo. Throughout the school year, agriculture department staff meetings are held weekly on Tuesday mornings before school - these meeting typically include the discussion of upcoming FFA activities, a briefing on SAE project areas, and discussion of any department needs/events. Weekly department meetings are occassionally done in a more casual manner over lunch, which results in deparment meeting minutes being avaiable for some, but not all meetings. This is something our deparmnet would like to address for the upcoming school year. In addition to weekly department meetings, campus wide staff meetings are held the first Thursday of each month, which is done during the "late-start" hour each Thursday classes begin at 8:45am rather than 8:00am, which allows for a built in meeting time. Along with campus wide staff meetings and department meetings, agriculture department staff usually attend "Team Meetings" within the PGHS Science Department on the second Thrusday of every month to ensure the Agriculture Department curriculum is aligned with the classes beign taught on main campus.

Chapter Officer Executive Committee Meetings are held each week during either the late start Thursday time period or after school if there is a staff or team meeting the FFA Advisor must attend. These Executive Committee Meetings allow the officer team to pan for upcoming FFA events and activities. 
A

Quality Criteria 5:

Facilities, Equipment

$\theta$ $\&$ Materials 


\section{Pleasant Grove High School Agriculture Department}

\section{Quality Criteria 5: Facilities, Equipment, and Materials}

The facilities, equipment, and materials at Pleasant Grove High School's agriculture department are fairly new, being constructed within the past two years. They represent an example of what students would see in a true agriculture operation, and are avaialbe for all students to utilize.

The agriculture classrooms are both identical, and include a large teacher work station, eight student lab stations for labs or hands-on activities, and a desk for each student. Additionally, each classroom has an integrate teachnology system, which allows for a one touch operation of the overhead project, vcr/dvd player, document camera, and an auxilary input. The main agriculture building also includes a department office, as well as a project/meeting rooms. All classrooms, barns, greenhouse, and floral cooler are up to date on current code to allow access to students with disabilities.
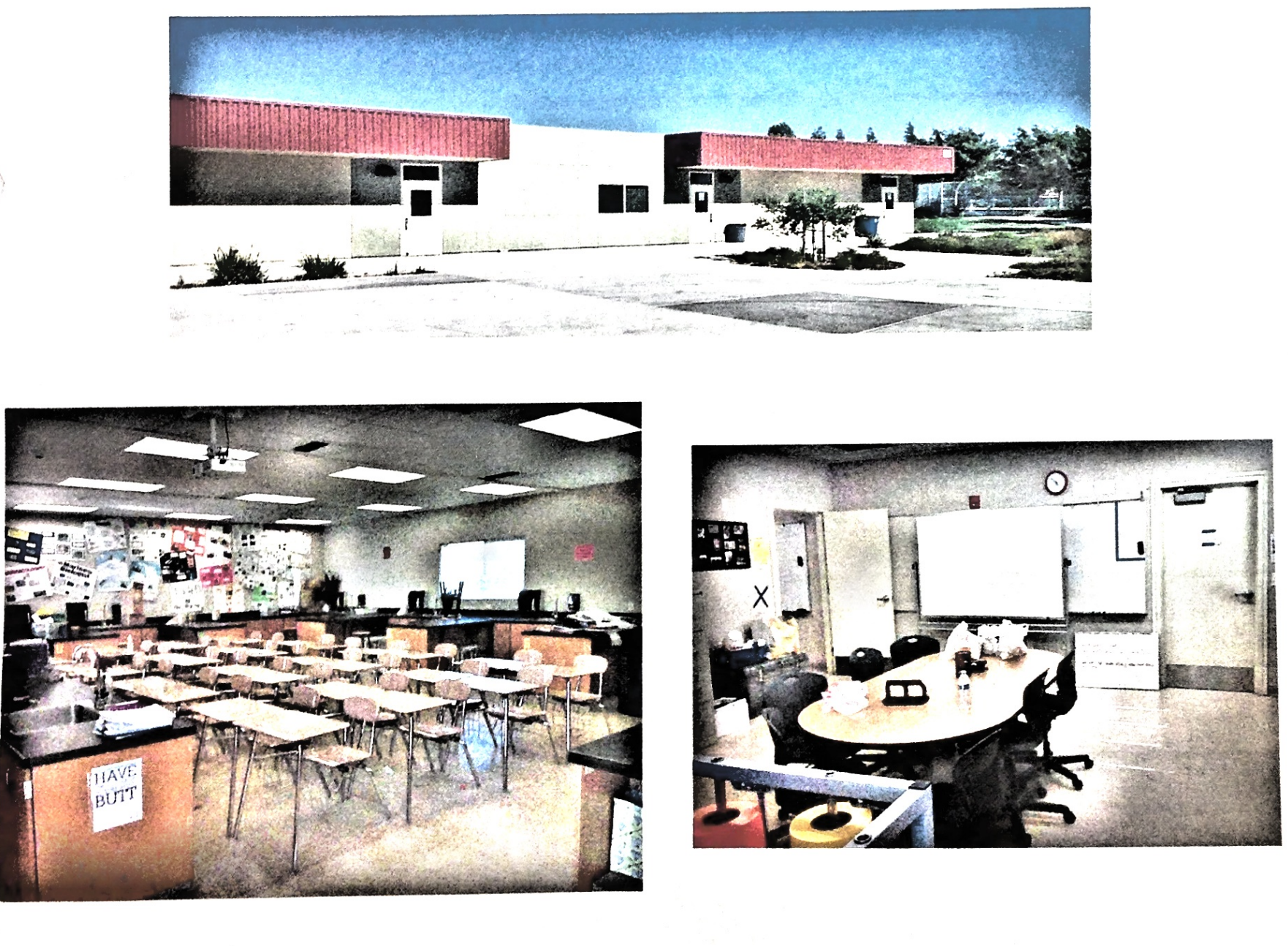
Located next to the agriculture classrooms is a free-standing, commercial size floral cooler. This allows for the processing and storage of large quantities of flowers which are used for both the Floral Design classes, as well as for floral arrangements completed for large district events such as graduation ceremonies held at the Sleep Train Arena.

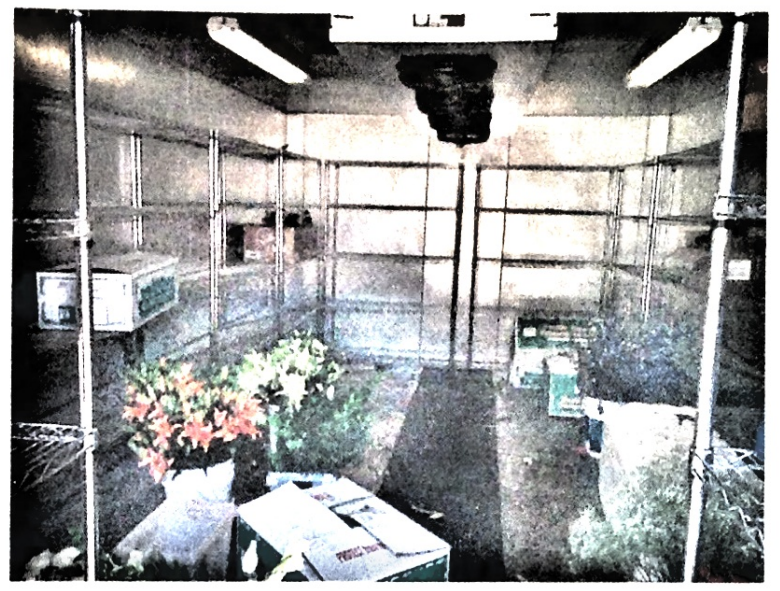

Next to the floral cooler is our newly constructed greenhouse. While not yet open and operational, construction is scheduled to be completed in mid July of 2014. This greenhouse includes a large growign area complete with metal growing tables, and a water and cooling system to control the environment for growing plants. Additionally the second half of the greenhouse as a separate climate controlled room which is designated as our third classroom at the agriculture facility. Eventually, this room will hold lab tables for students to work at in addition to a large teacher instruction stations. We are working with one of our current students to have a shade house constructed next to the greenhouse as part of an Eagle Scout Project once the greenhouse has been completed.

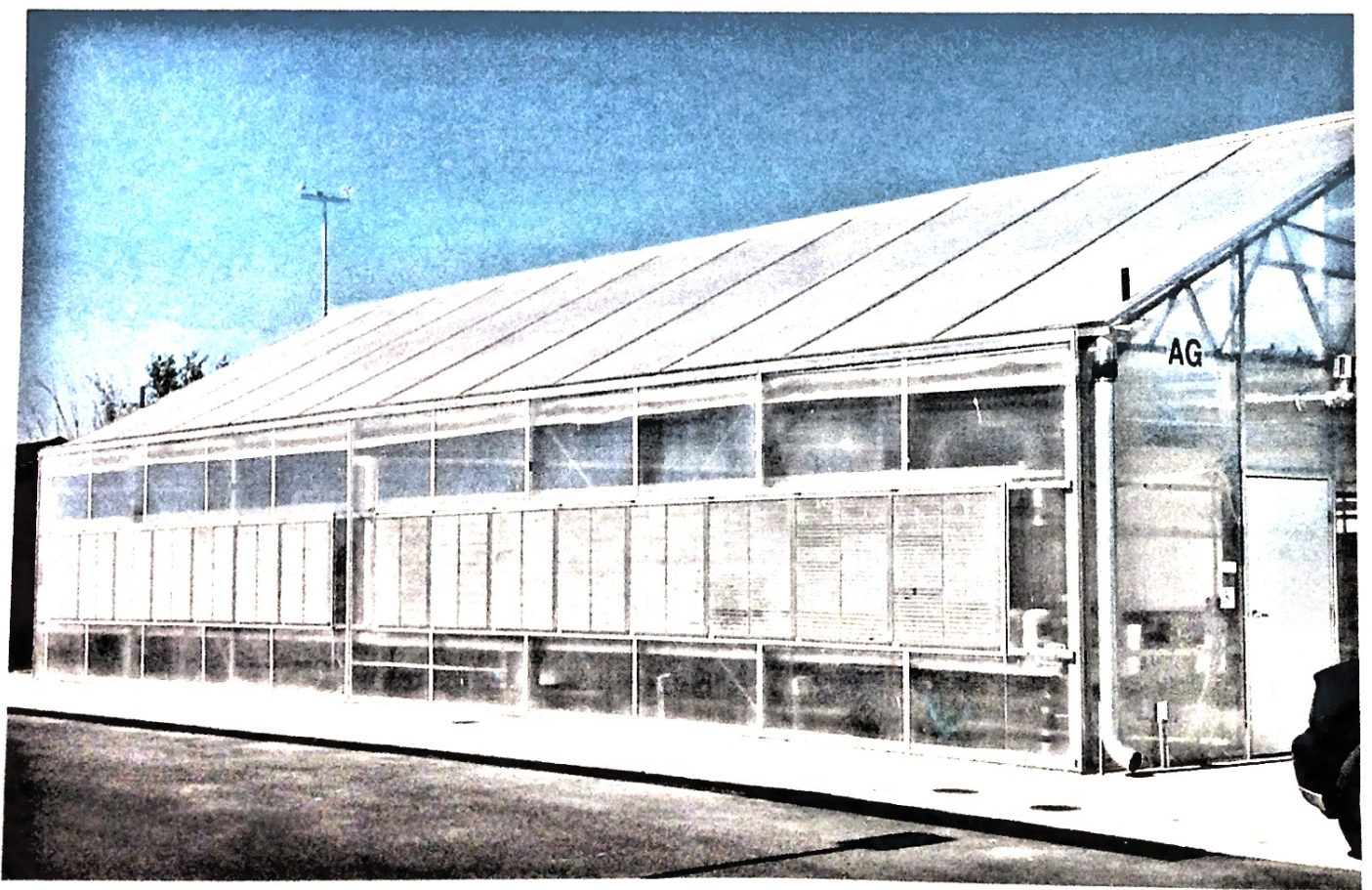


Our large and small animal barns are also newly constructed, and includes space for cattle, hogs, lambs, goats, poultry projects, and a small rabbitry area. The large animal barn contains two wash racks for fair animlas, an equipment storage area, and a feed area for all species. There are also two livestock scales for hogs, lambs, and goats as well as large scale which can be pulled behind the school's agriculture truck for weighing cattle projects. The large animal barn also features two large exercise lots for animals, which are also used as showmanship practice areas as the fair appporaches. The small animal barn contains a dirt area for poultry projects as well as a small chicken coop for students' laying hen projects, as well a cement half where market rabbit projects are kept for the Sacramento County Fair. The school farm also contains a small orchard of 8 fruit trees, as well as a storage container for any livestock and farm equipment.
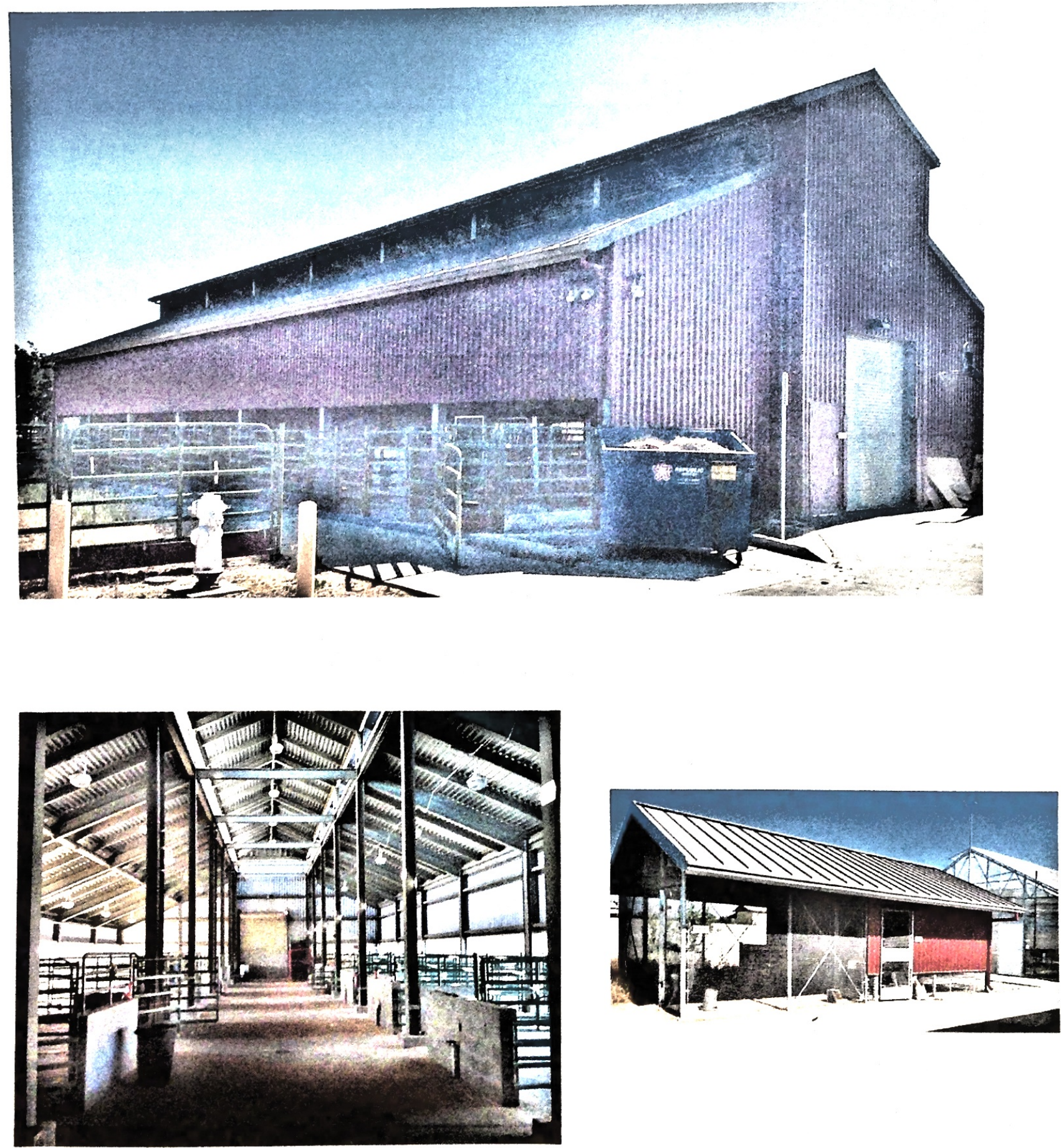


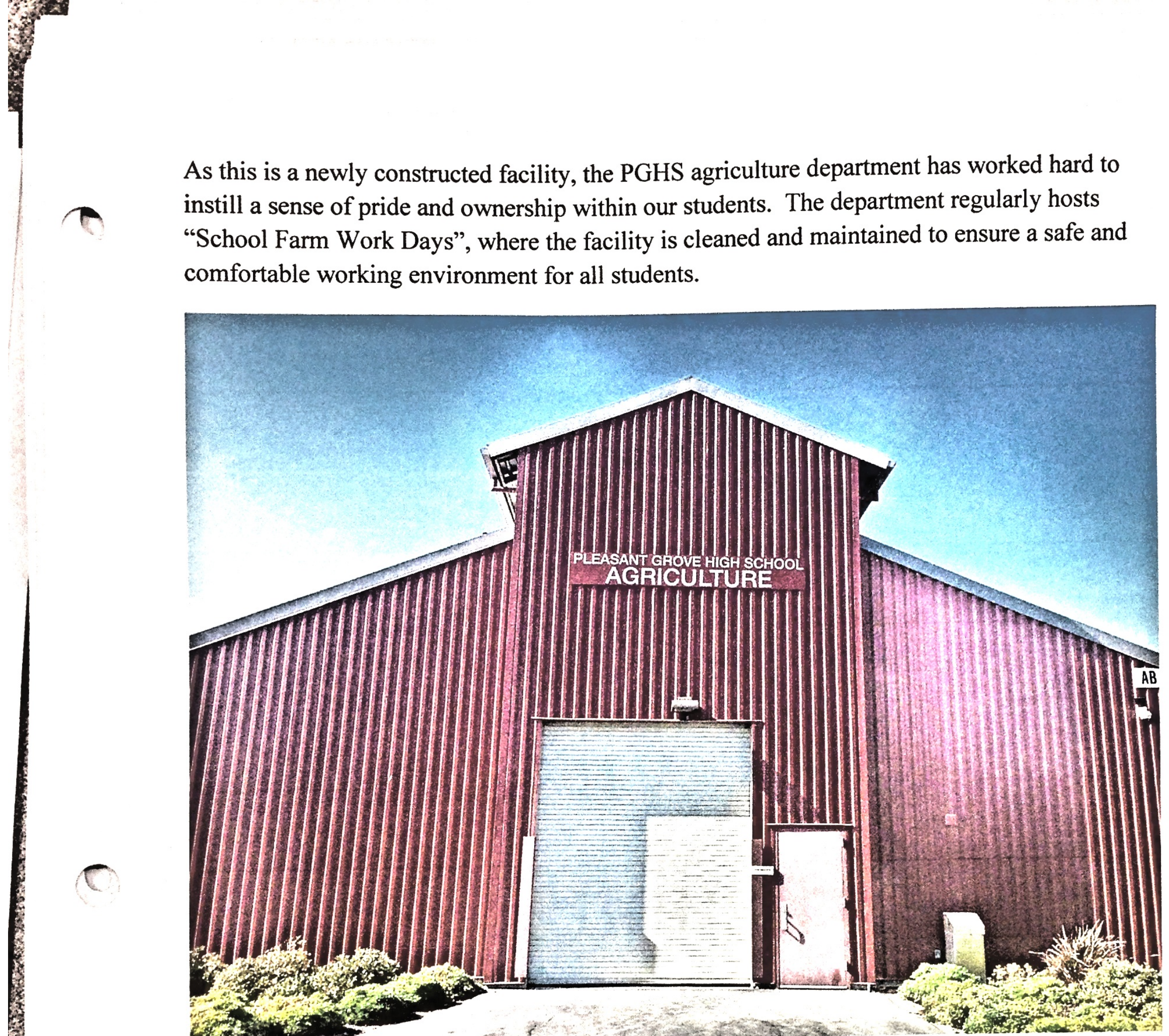


6

\title{
Quality Criteria 6: \\ Community, Business,
}

\author{
$\&$ Industry \\ Involvement
}




\section{Pleasant Grove High School Agriculture Department}

\section{Quality Criteria 6: Community, Business, and Industry Involvement}

The Agriculture Advisory Committee at Pleasant Grove High School is made up of community leaders who represent local businesses, the commercial agriculture industries, parents, students, staff, and district personnel. The Advisory Committee meets at least twice each school year, and occassionally a third meeting is held if necessary. As our agriculture facility has been building and constructed, the Advisory Committee as been instrumental in assisting the department with ensuring our program and facility are meeting the needs not only of our students but of the agriculture industry as a whole. They provide practical advise and guidance on how best to improve our program for the betterment of our students and the Elk Grove community.

The Advisory Committee plays as active role in assisting with the development of the 5 year Facility and Equipment Acquisition plan, our Graduate Follow Up, and department budgets. Their experiecne and expertise allow the PGHS agriculture instructors to have a constructive outsdie perspecitve and assists in building connections throughout the Elk Grove community that allow us to fulfill the program requirements present in the Agriculture Incentive Grant Checklist. 
Quality Criteria 7: Career Guidance 


\section{Pleasant Grove High School Agriculture Department}

\section{Quality Criteria 7: Career Guidance}

All studnets enrolled in an agriculture class at PGHS complete a Student Data Sheet that is completed at the beginning of each school year. Student Data Sheets are kept in student files for the duration of their enrollment in the agriculture program, and are maintained for three years post graduation.

Students, staff, and counselors are kept up to date about the opportunities availabe to students after they graduate from PGHS. In each of our agriculture department classes, students participate in a Careers in Agriculture Unit, which focuses on agricultural careers related to the specific course they are enrolled in. Additionally, all students are also informed about their education and career options upon leaving PGHS as well as any scholarship opportunities. In our $12^{\text {th }}$ grade course Economics in Agriculture, students are also visitied by students from our local community colleges that feature agriculture programs as well as local techincal schools
such as UTI.

Currently, our agriculture department has an articulation agreement with Consumnes River College in Sacramento with our Economics in Agriculture class. This gives our economics students the opportunity to earn credit for an Agriculture Business class if they choose to attend Consumnes River College. 


\section{Quality Criteria 8: \\ Program Promotion}




\section{Pleasant Grove High School Agriculture Department}

\section{Quality Criteria 8: Program Promotion}

Recruitment is arguable one of the most important aspects to a successful agriculture program. At PGHS, we try to educate 4 groups of people about the opportunities available to students in our program - students, parents, school staff/administration, and the community

The first group of people we try to recruite is students. We have a standing agreement with our feeder school (conviniently built next to PGHS) to conduct a recuirment day in all $8^{\text {th }}$ grade sceince classes each March. Our student run Recruitment Committee is responsible for planning the activitis that take place at this recruitment day, which normally include a presentation on opportunities available to students in the agriculture department, shared personal stories of current FFA members, and fun activities that allow $8^{\text {th }}$ graders to connect with current students. Each $8^{\text {th }}$ grade classroom typically has four to five current agriculture students conduct the recruitment activities, which conclude with flyers with information for parents.

Once students have completed the recruitment day activities, our Chapter Officer Team attends the annual $8^{\text {th }}$ Grade Parent Information Night, where different departments present information to the $8^{\text {th }}$ grade parents. Chapter Officers give a five minute run-down of the opportunities avaialbe to students through FFA, and are availabe at the conclusion of the evening to answer any questions from parents.

One of the most important ways to recruit parents is through conducting Home Visits. Freshmen students are encouraged to schedule a Home Visit (and in some classes are required to do so), at which department and FFA information are shared, a plan is formulated for studnets' SAE projects, and any questions about the program can be addressed with the student and parent individually. In addition to Home Visits, parents are also kept up to date on department activites through regular emails sharing upcoming activities and student achievements, and are encouraged to join our newly formed Aggie Backers (a parent based booster group).

To retain our current studetns, we conduct planning sessions in all agriculture classes to ensure students know their options for each grade level, where next year's class schedule is planned, to ensure they are meeting all graduation, higher education, and program requirements. These year plans are required to be signed by a parent/guardian to make them aware of options through the agriculture program.

Lastly, we education our faculty, staff, administration, and community about our program to ensure support for our students. Back to School Night allows counselors, administrators, and School Board members to see the great things our students are accomplishing. Our Department Chair also attends all Steering Meetings to represent our department and education staff and administrations. Our Advisory Committee has been instrumental in helping us become more visible in the Elk Grove community though various activities to keep our program growing. 
Quality Criteria 9: Program

Accountability \& Planning 


\section{Pleasant Grove High School Agriculture Department}

\section{Quality Criteria 9: Program Accountability and Planning}

A Comprhenisve Program Plan is on file with our Regional Supervisor, Ms. Jean Landeen, and a copy of this plan is kept within our department to be updated each year. Updates are sent to Ms.Landeen each year by November $15^{\text {th }}$, as required by the Agriculture Incentive Grant. Each year our department reviews the following items: Five Year Equipmetn Acquisition Schedule, Chart of Staff Repsonsbilities, Advisory Committee Roster, and Advisory Committee Minutes. We also submit our Graduate Follow Up Date, R-2 Report, AIG Expenditure Report, and FFA Roster each year by October $15^{\text {th }}$ in accordance with the requirements set forth. Every three years, our Comprehensive Program Plan is also reviewed by Mr. Hugh Mooney, as a representitive of the California Department of Education.

Each year our department chair receives our tentative enrollment numbers for the following school year in mid to late May. After we have an idea of our program numbers, we then complete our "Teaching Wish Lists" forms, which indicate which classes we would like to teach, when we would like a prep period, etc. It is imperative that we complete these forms as quickly as possible to ensure that we can have the needed classes for our students incorporated within the Master Schedule for PGHS. 
Quality Criteria 10:

- Student-Teacher Ratio 


\section{Pleasant Grove High School Agriculture Department}

\section{Quality Criteria 10: Student-Teacher Ratio}

Like many agriculture programs in our district and throughout the state, we struggle to maintin the proper student-teacher ratio for class instruction as required by the Agriculture Incentive Grant. While we do maintain higher than recommended numbers, I believe this may be a testament to popularity of our agriculture classes and the growing number of students within our progarm. Even with over the "limit" class sizes, each instructor does their best to provide relevant and rigourous material in all classes. Unforntunatley our department is not allocated with any project supervision periods, so all SAE supervision is done after school hours. We are well over the suggested 75 students per instructor, but we happy to see progarm growth despite budget cuts and restriction on adding staff members. It is our hope that we will be able to increase our staff numbers in the future to hopefully balance out the student-teacher ratio to hopefully eliminate the cut in our Agriculture Incentive Grant requirements.

Student-Teacher Ration Averages

\begin{tabular}{|c|c|c|c|}
\hline Class & Forster & Swenson & Zallo \\
\hline $\begin{array}{c}\text { Applied General } \\
\text { Science }\end{array}$ & $27: 1$ & $35: 1$ & \\
\hline Agriculture Biology & & $32: 1$ & $29: 1$ \\
\hline Floral Design & $31: 1$ & & $33: 1$ \\
\hline $\begin{array}{c}\text { Animal Anatomy \& } \\
\text { Physiology }\end{array}$ & & & \\
\hline
\end{tabular}

Current Enrollment Numbers

\begin{tabular}{|c|c|c|c|}
\hline Class & Forster & Swenson & Zallo \\
\hline $\begin{array}{c}\text { Applied General } \\
\text { Science }\end{array}$ & 27 & 92 & \\
\hline Agriculture Biology & & 32 & 85 \\
\hline Floral Design & 116 & & 67 \\
\hline $\begin{array}{c}\text { Animal Anatomy \& } \\
\text { Physiology }\end{array}$ & & & \\
\hline
\end{tabular}


Quality Criteria 11:

Full Year Employment 


\section{Pleasant Grove High School Agriculture Department}

\section{Quality Criteria 11: Full Year Employment}

Currently PGHS and the Agriculture Deparment do not have full year employment. Our district is extremely large, and features four large agriculture programs, which makes budgeting for a summer stipend impossible at this time. Each year, instructors have the option to take advenatage of teaching a Summer ROP Class, which equates to 35 non-contract days, and a stipend of $\$ 10,000$. Currently, only one instructor takes advantage of this option on campus, and uses to supervise student swith year round SAE projects and any students taking projects to the California State Fair. Due to budget contrainsts within our district, we currently do not have an FFA stipend, nor a project supervision period, which we will most likely not be able to secure due to budgeting issues. 


\section{Section II}

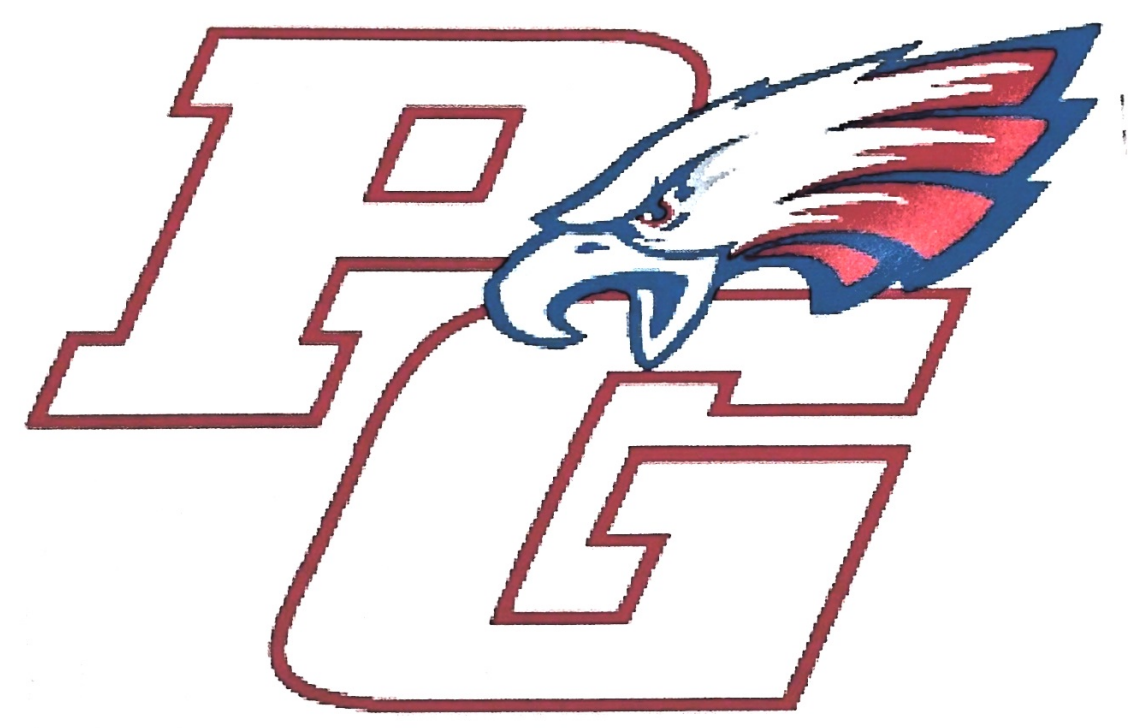

Home of the Eagles

Support Materials 


\section{Table of Contents}

\section{Section II: Support Materials}

Support Material 1: Student Data Sheets

Support Material 2: Permanent Student File

Support Material 3: Agriculture Course Outlines

Support Material 4: Course Gradebooks

Support Material 5: Completed SAE Supervision Forms

Support Material 6: SAE Project Statement in Syllabus

Support Material 7: FFA Statement in Syllabus

Support Material 8: FFA Program of Activities

Support Material 9: Recruitment Program

Support Material 10: FFA Chapter Scrapbook

Support Material 11: Summer Activities Calendar

Support Material 12: Graduate Follow Up Survey

Support Material 13: Graduate Follow Up Survey Results

Support Material 14: Comprehensive Program Plan

Support Material 15: Advisory Committee Meeting Agendas

Support Material 16: Advisory Committee Meeting Minutes

Support Material 17: Advisory Committee Constitution \& By-Laws

Support Material 18: Proficiency Standards

Support Material 19: Teaching Credentials

Support Material 20: Calendar of Activities

Support Material 21: Professional Growth \& Development

Support Material 22: R-2 Report

Support Material 23: Travel Request

Support Material 24: CATA Membership Card

Support Material 25: Report to Administration

Support Material 26: Five Year Acquistion List

Support Material 27: Operating Budget for Department

Support Material 28: Budget Process

Support Material 29: Chair Person's Duties \& Responsibilities

Support Material 30: Chart of Responsibilities

Support Material 31: Substitue Teaching Procedure \& Plans

Support Material 32: Description of Program Completer

Support Material 33: Articulation Agreement with Consumnes River College

Support Material 34: Reimbursement Process 


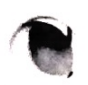

Support Material 1: Student Data Sheets 


\section{Pleasant Grove High School Agriculture Department}

\section{Support Material 1: Student Data Sheets}

Student Data Sheets are completed at the beginning of each school year in all PGHS Agriculture Deparment classes. A copy of this sheet is kept in students' data files, and maintained for three years post graduation. 


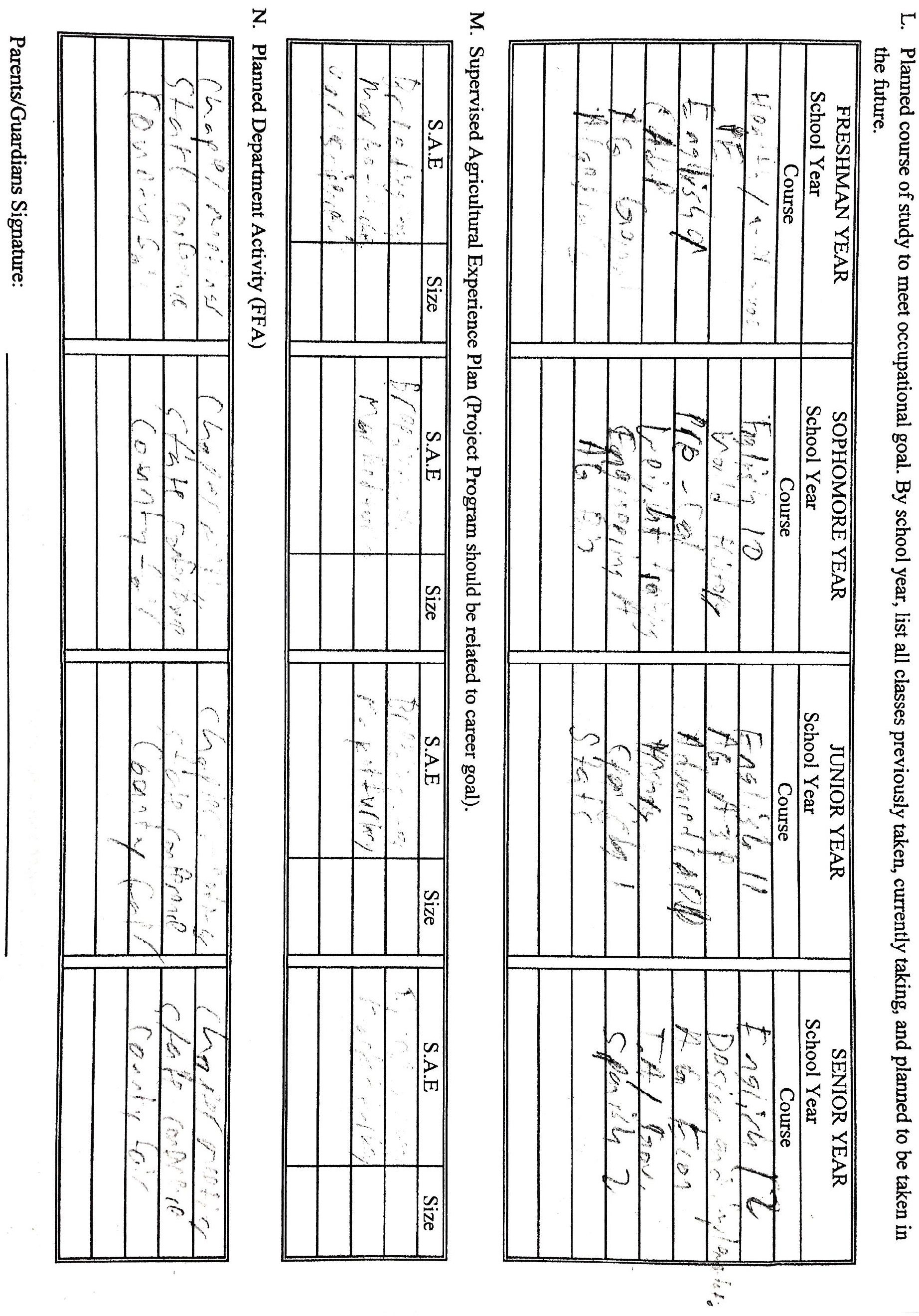




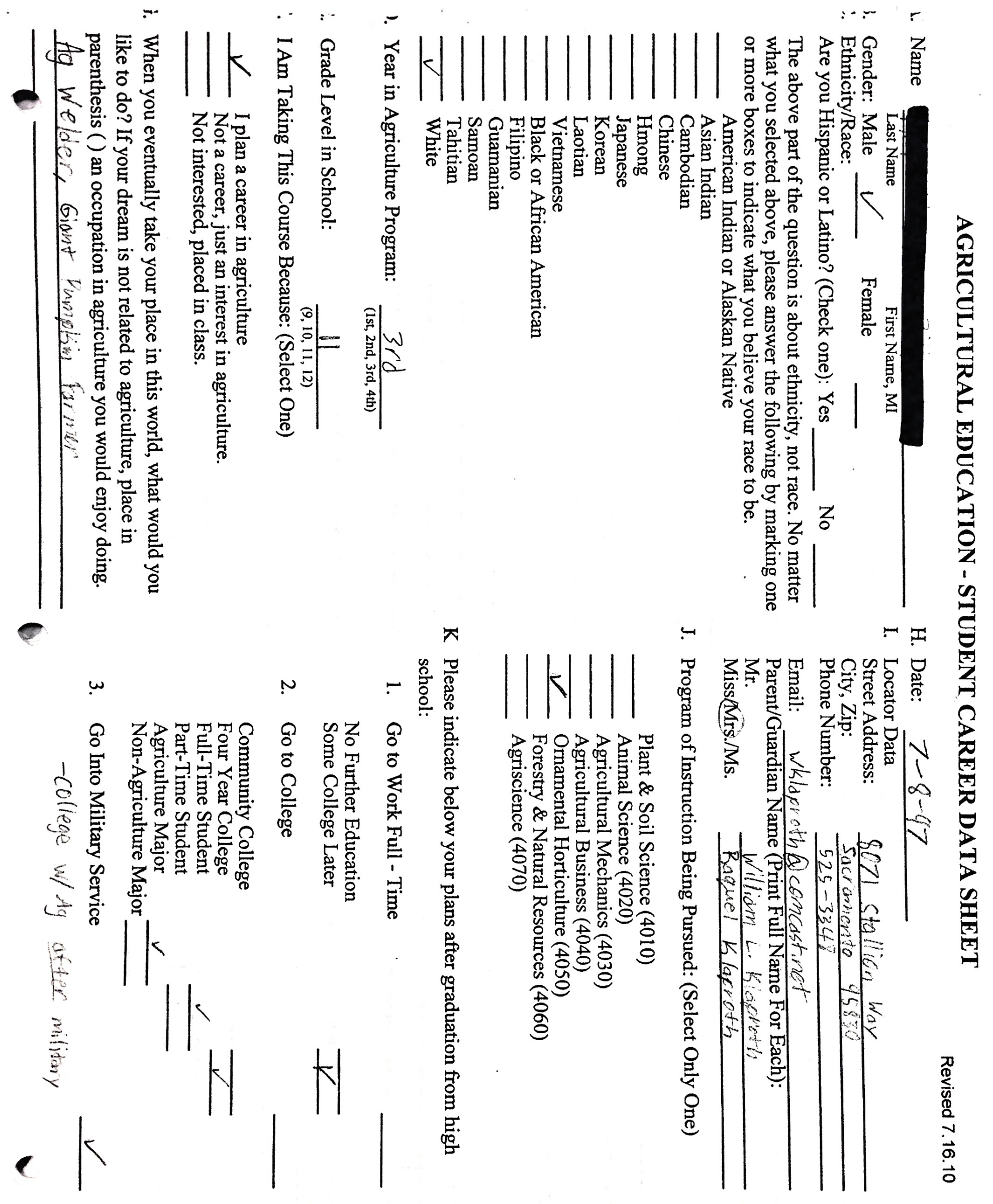




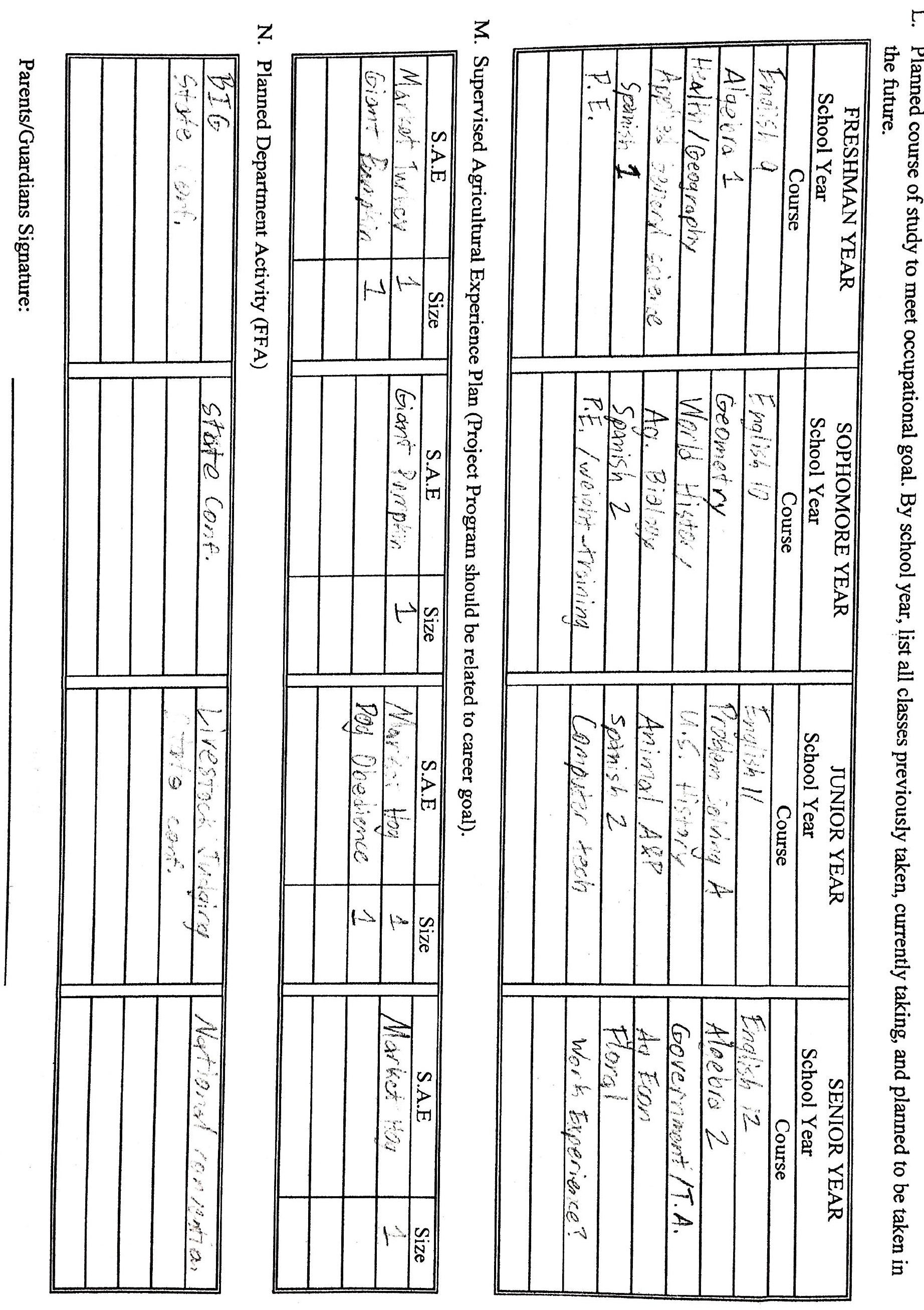




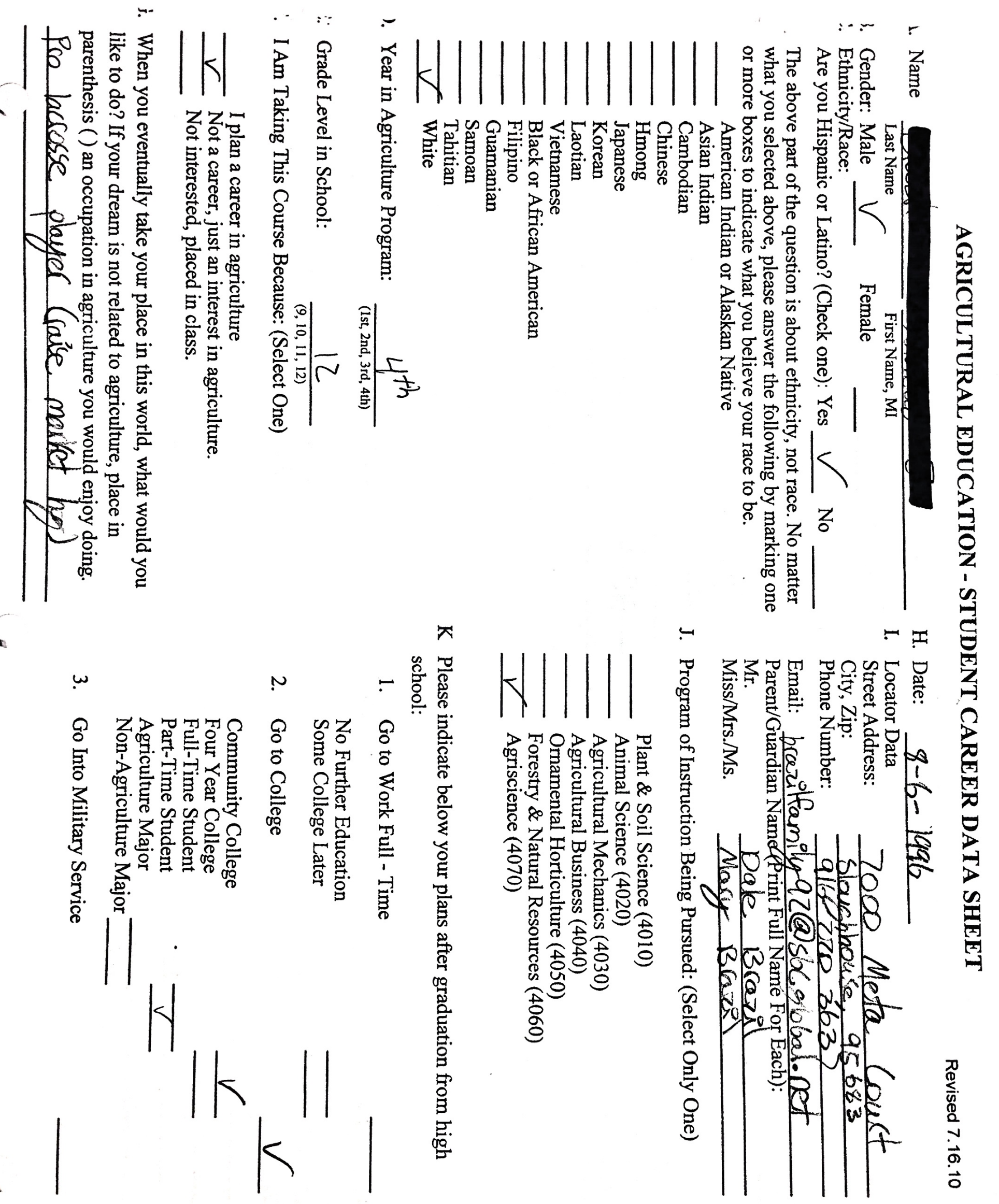



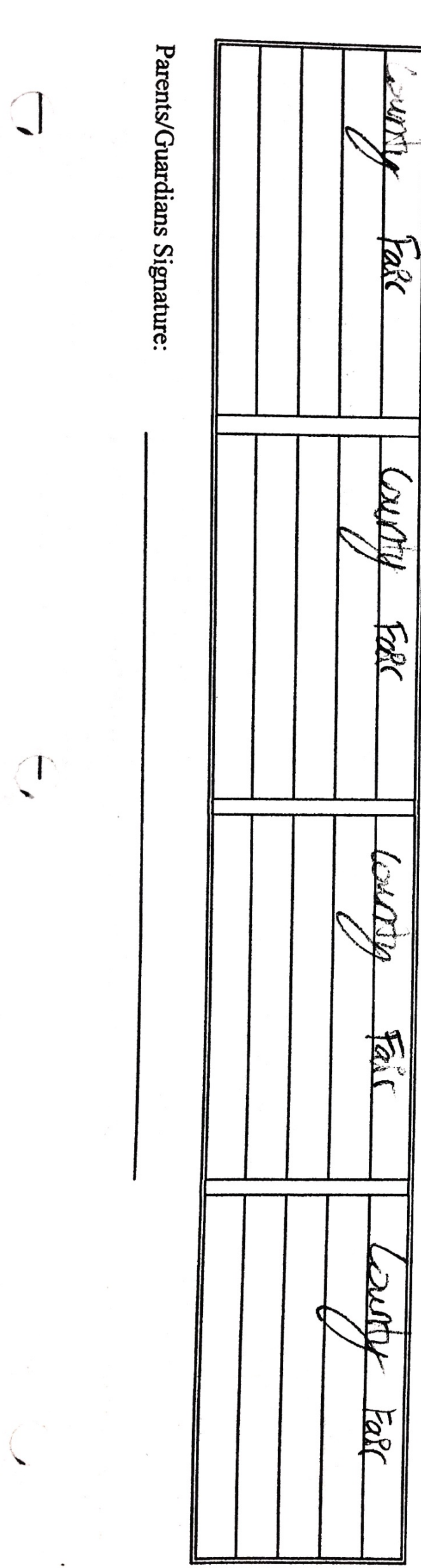

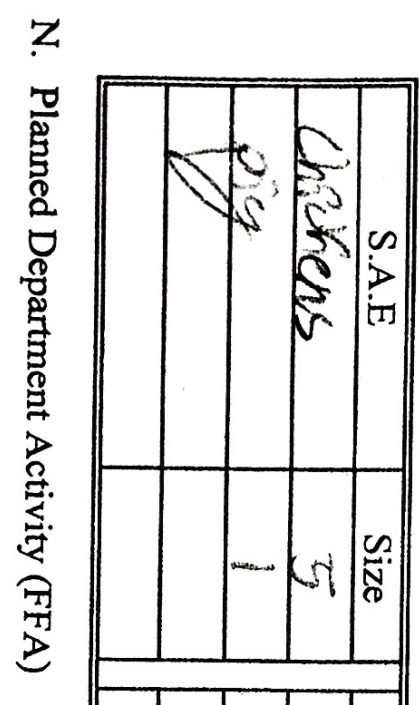

3

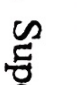

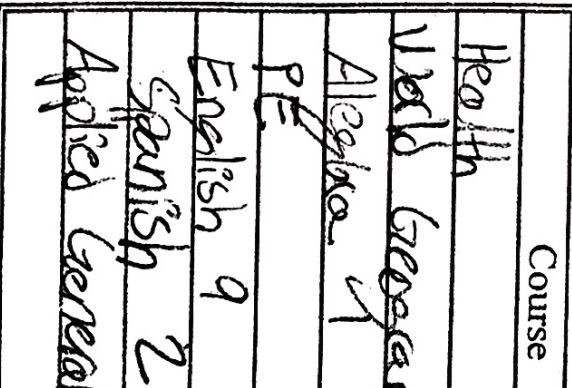

5 $-S$ S n

atc $A$ $\sqrt{2} \sqrt{2}$ co
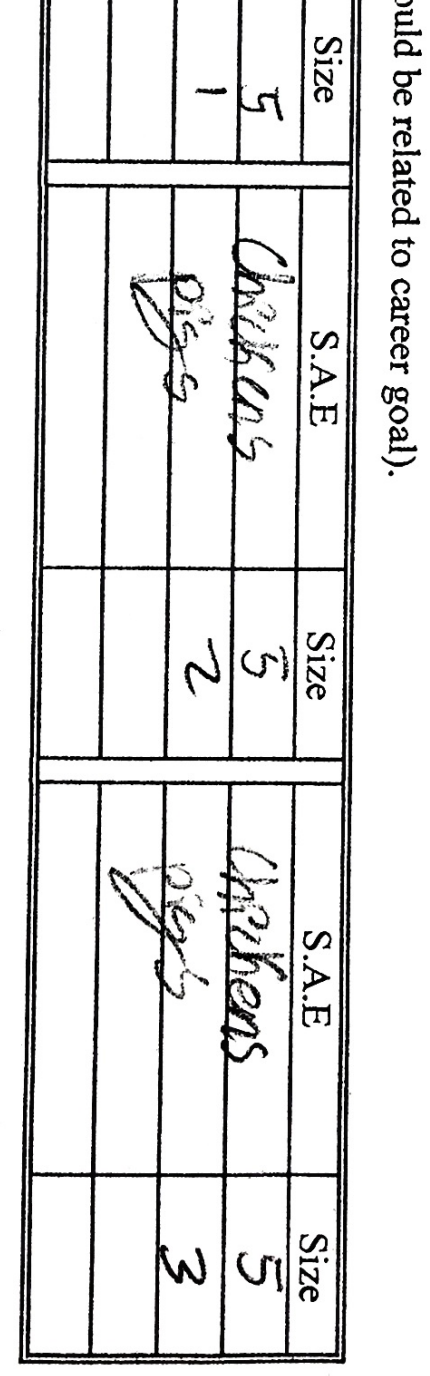

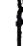
$\int^{\infty}$

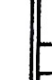
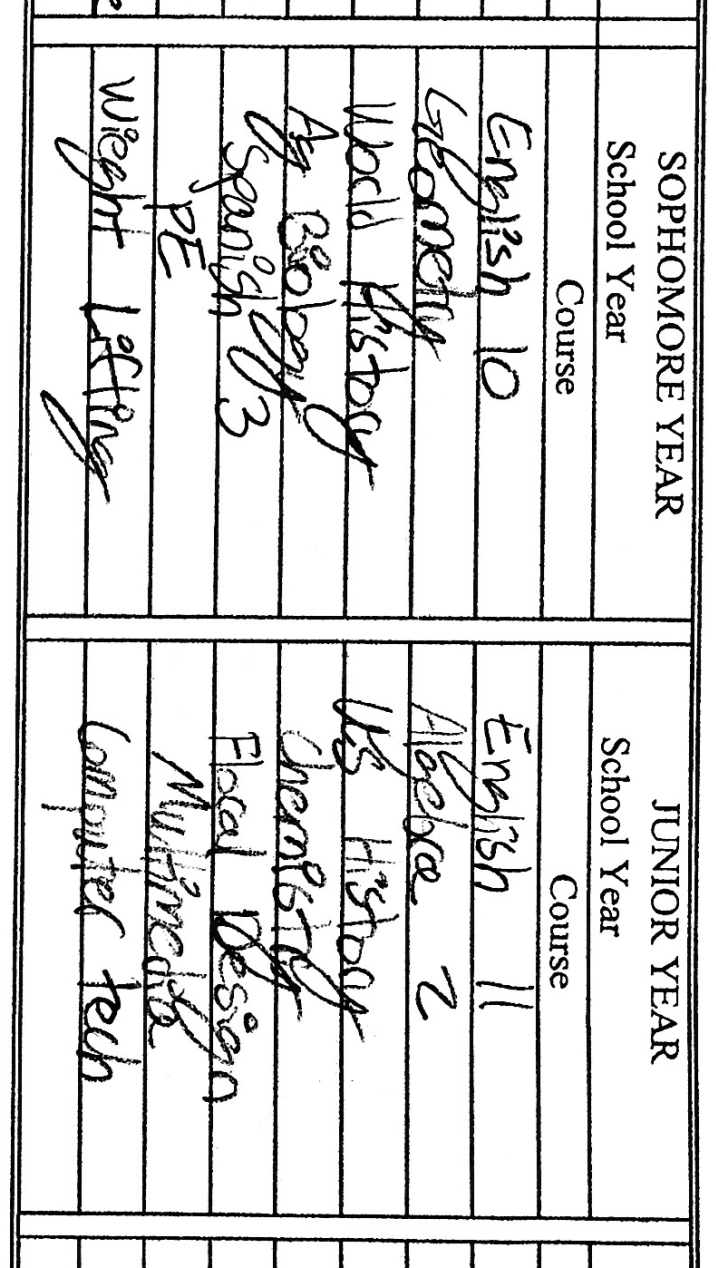

$\rightarrow 5 \frac{5}{5}$

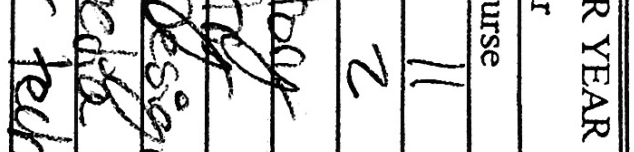

둥

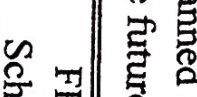
젱 8 焉 $\stackrel{7}{\circ}$

芑

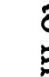

$\stackrel{\mathbb{I}}{+}$

ஜ્ટ

总.

总

\%o

W్

岇

๕

范

(

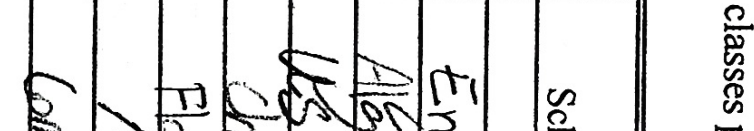

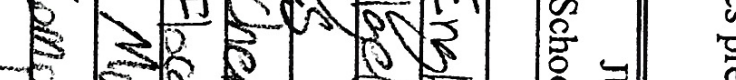

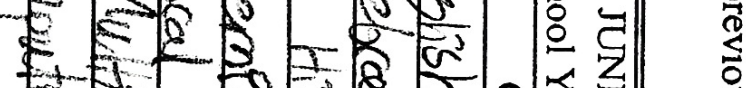

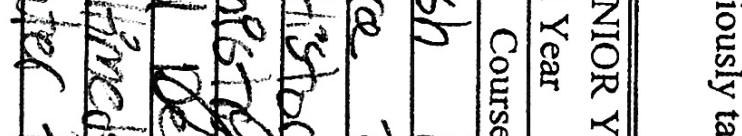
3500

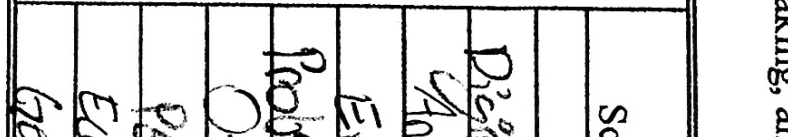

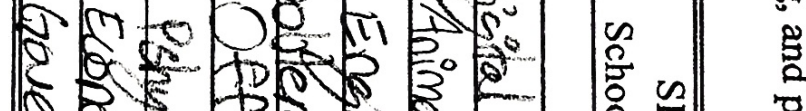

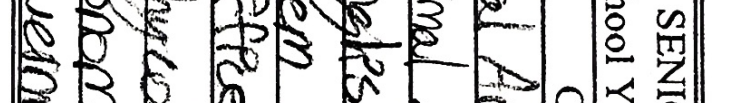

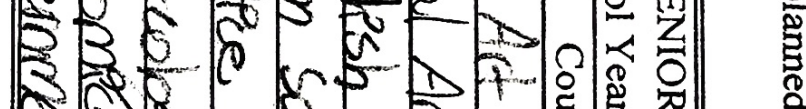

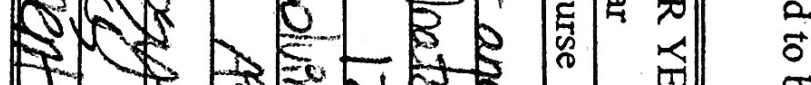
7 की

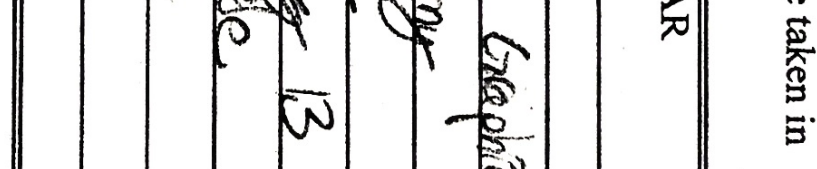




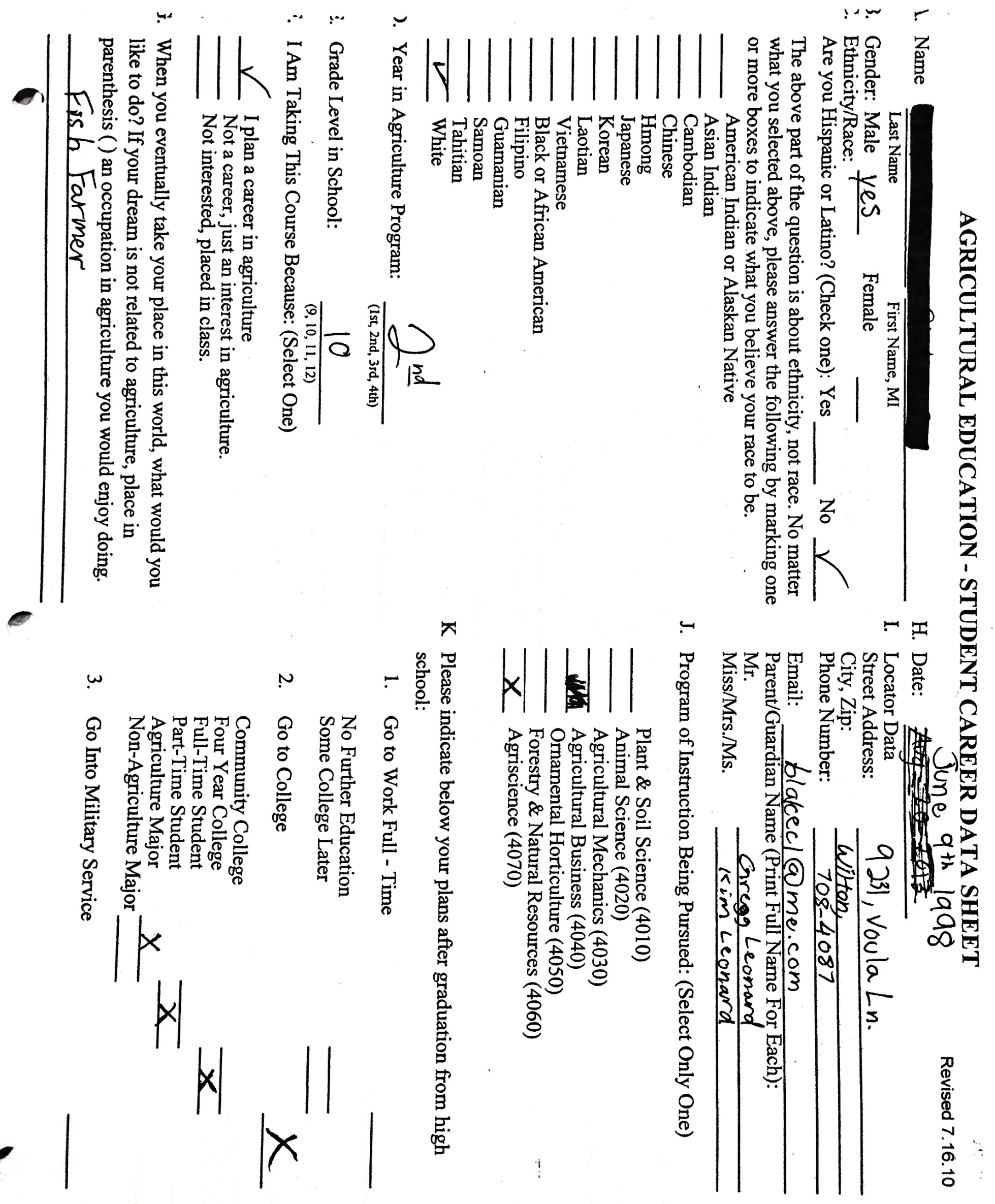




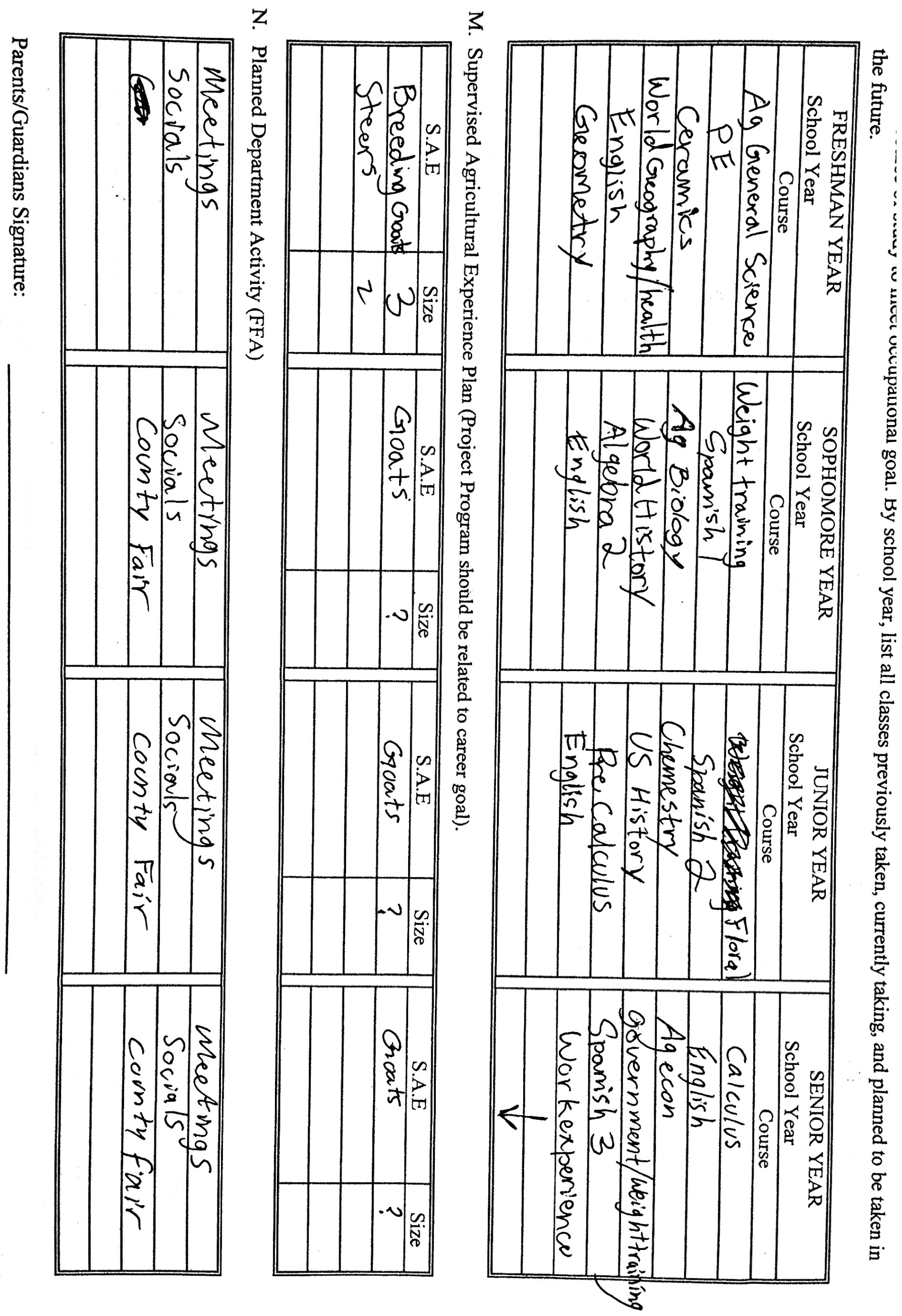




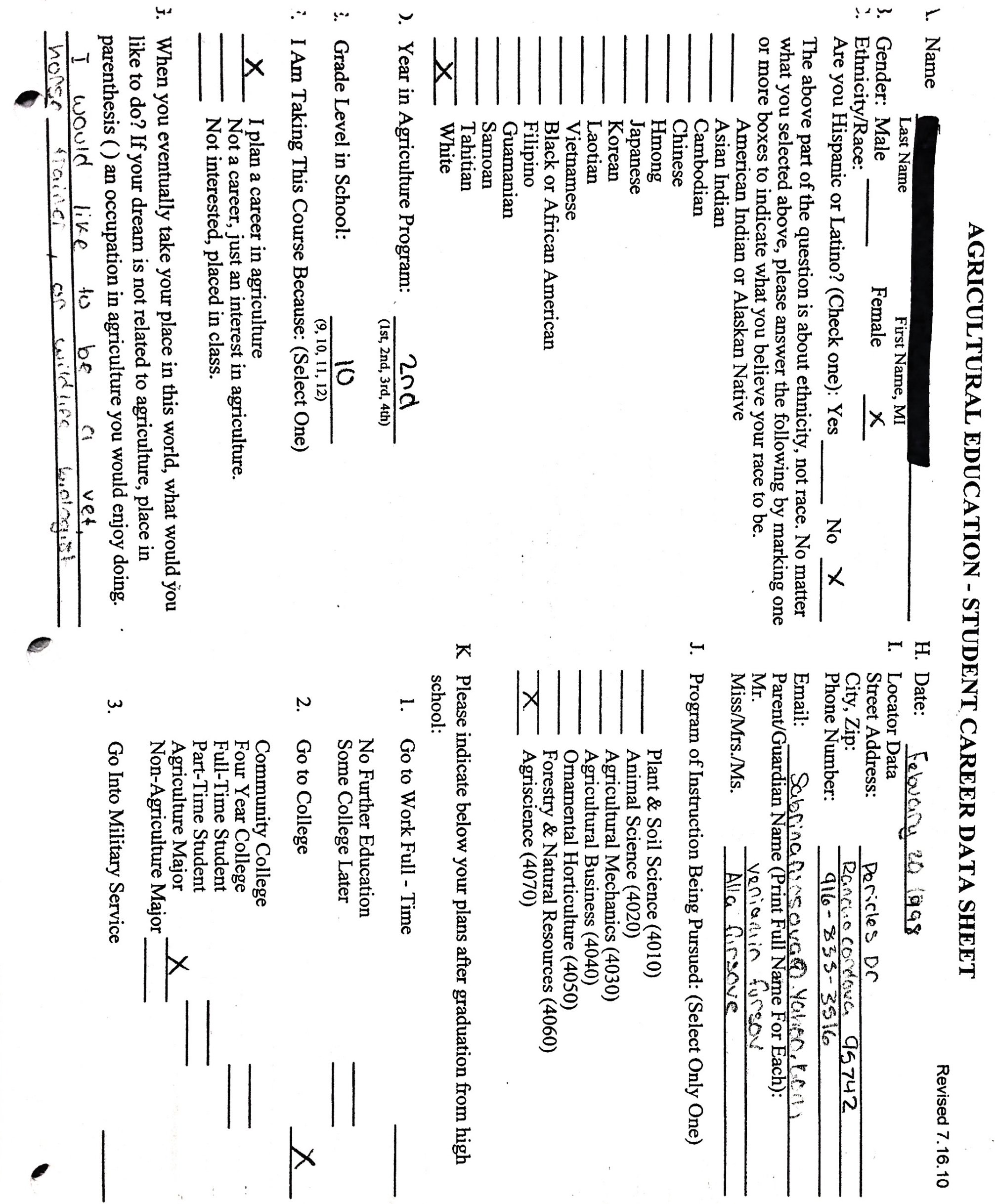





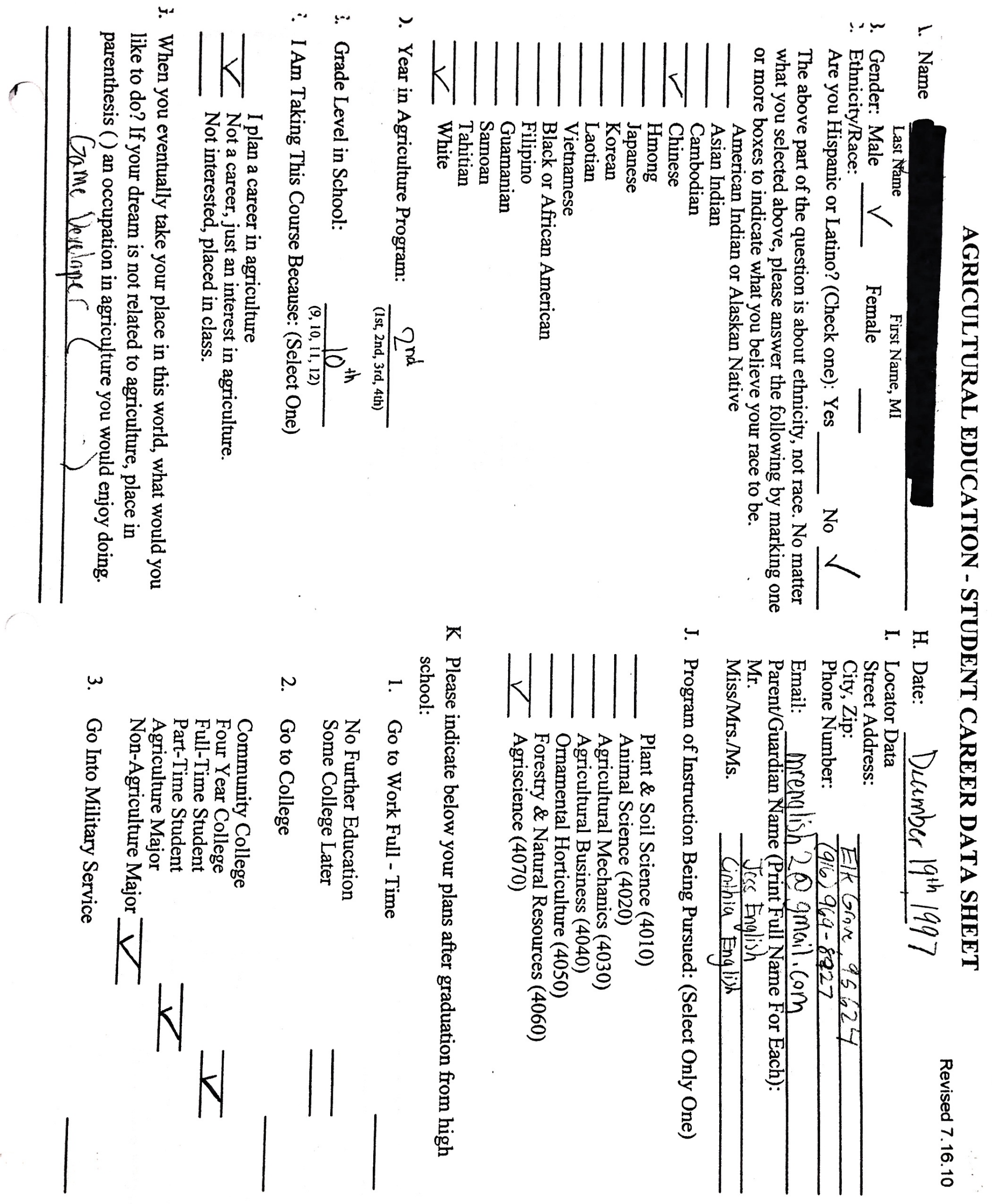




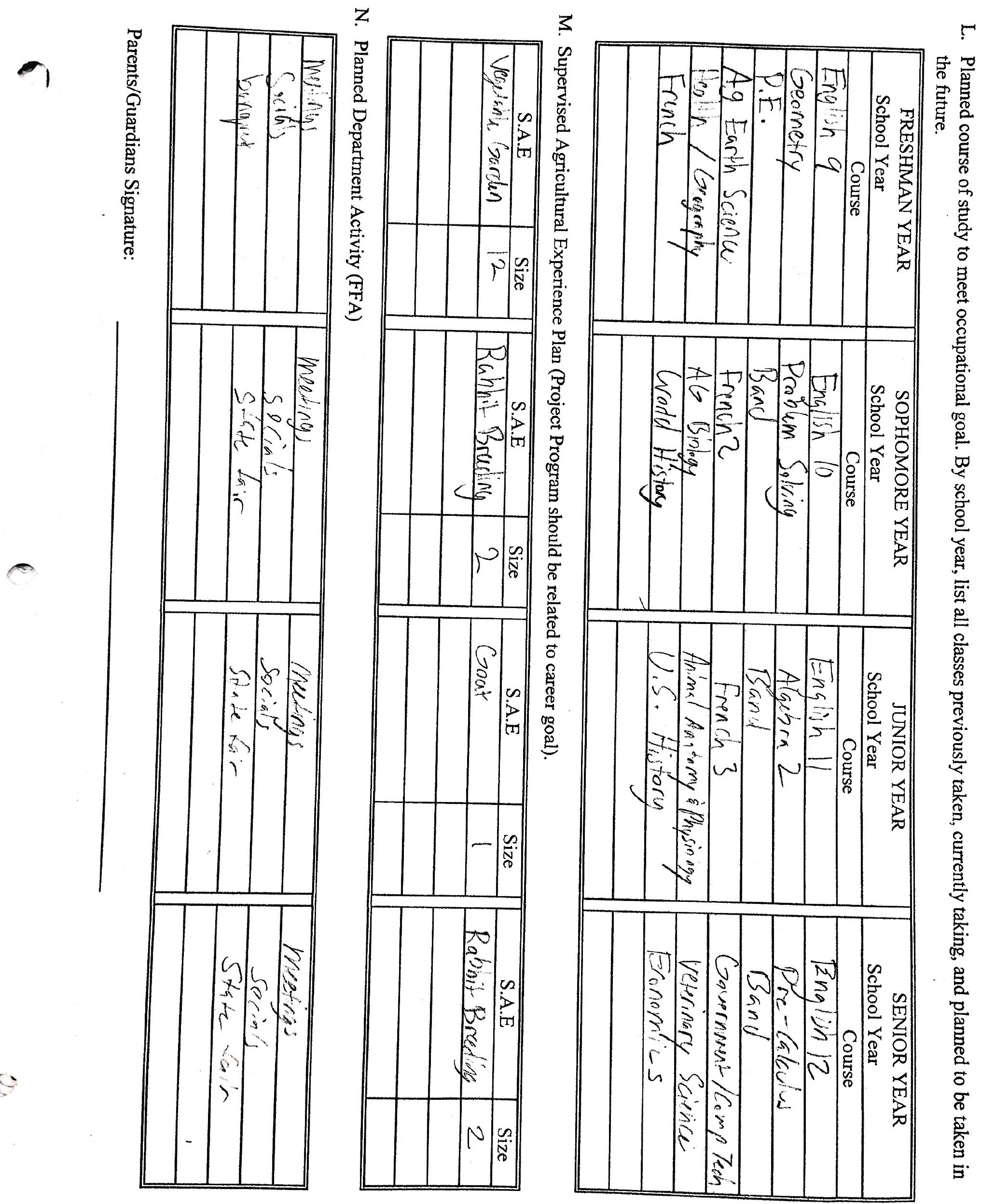




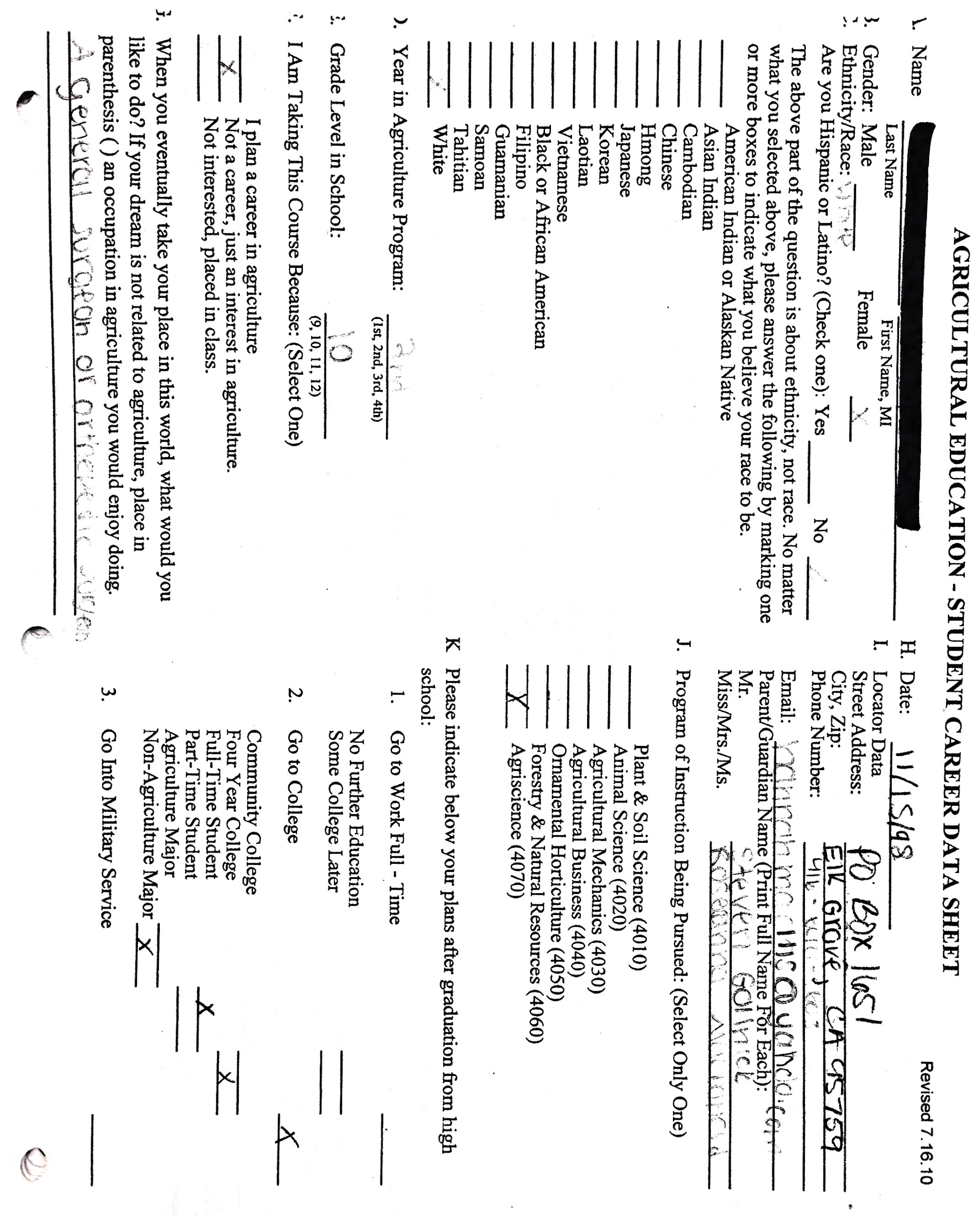



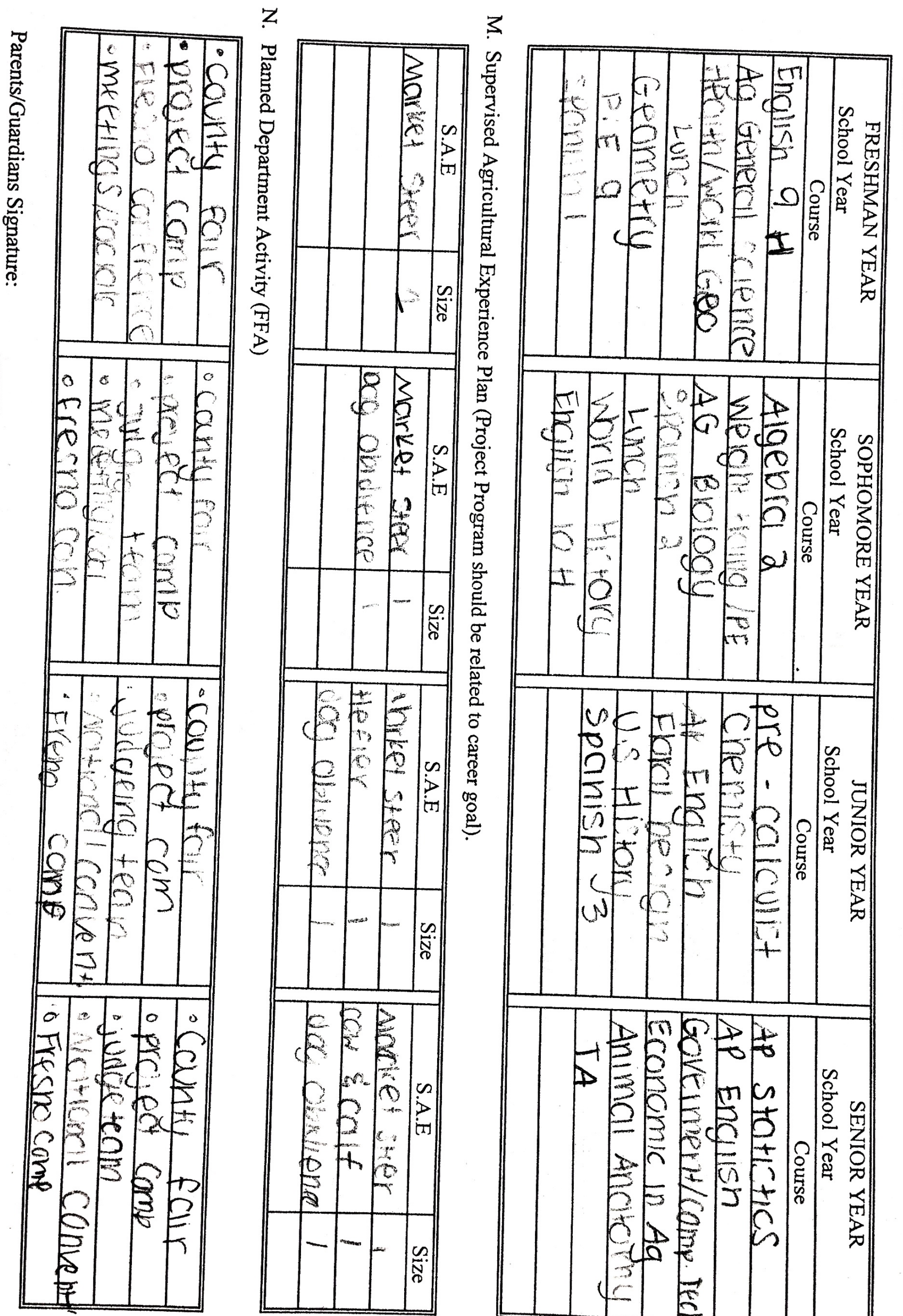

5

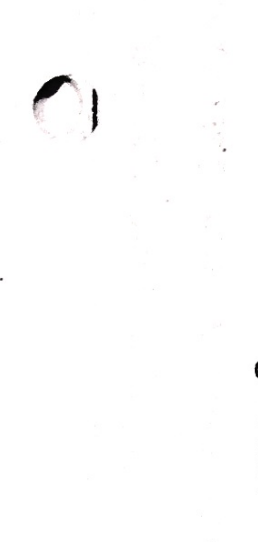




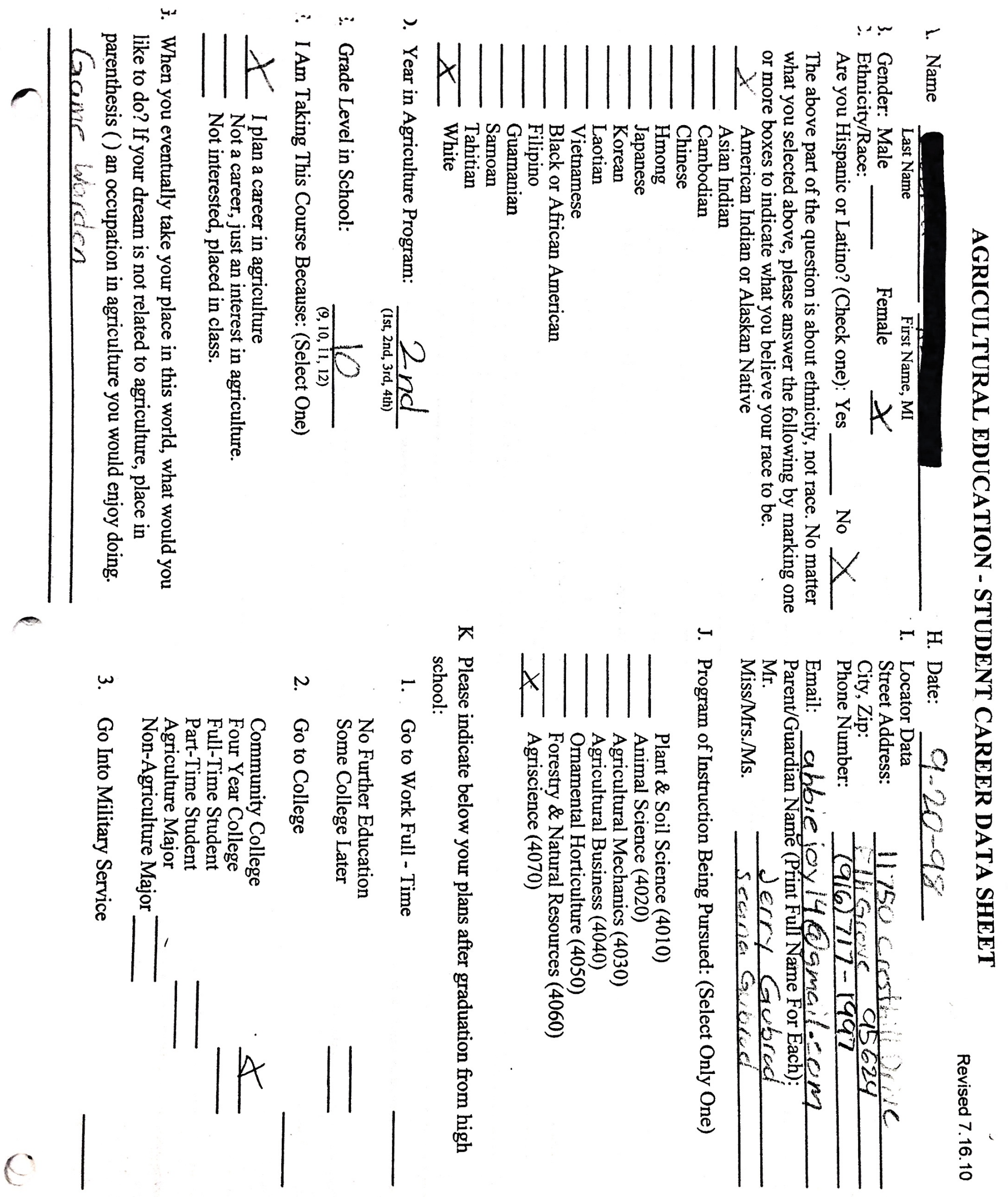




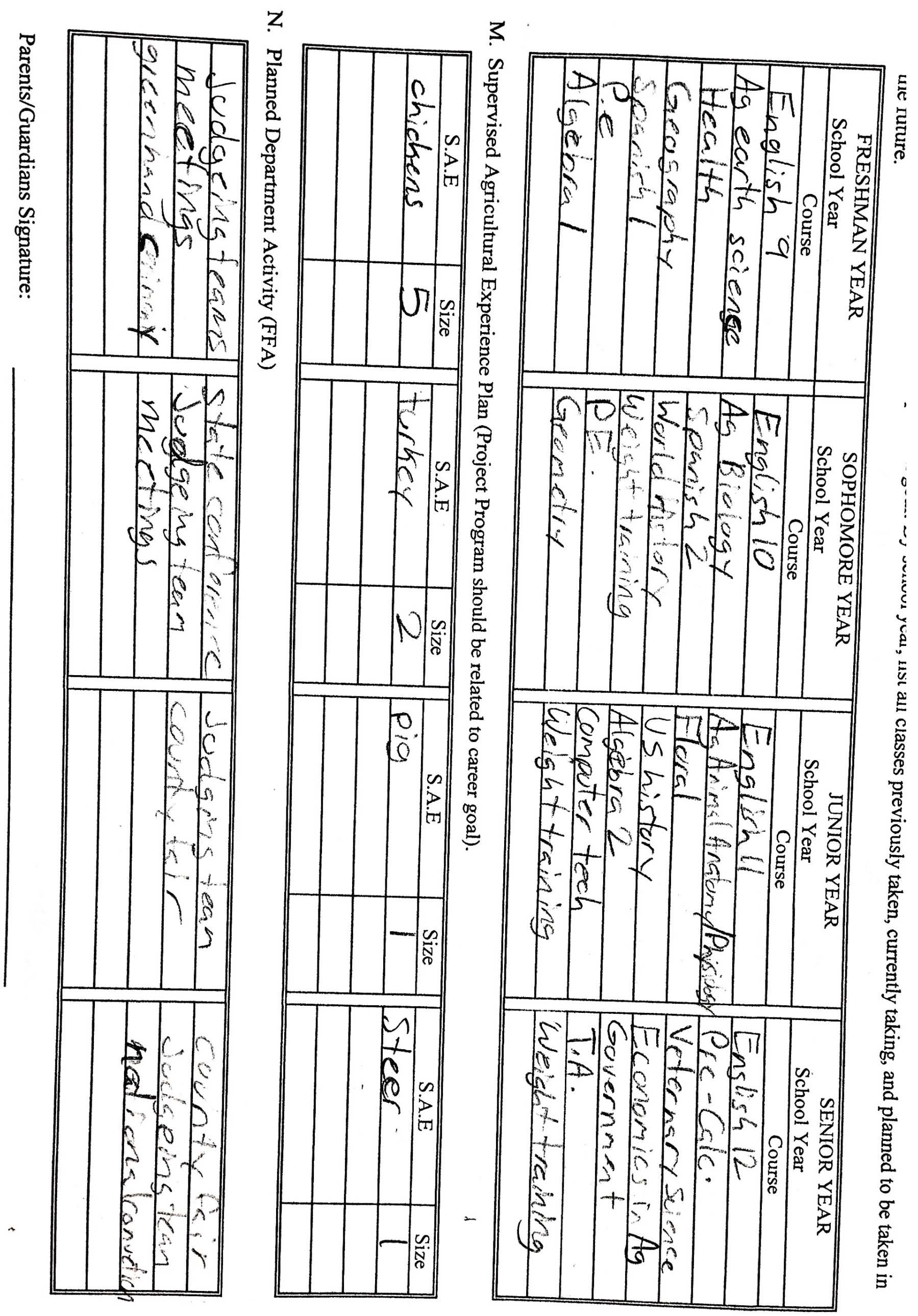




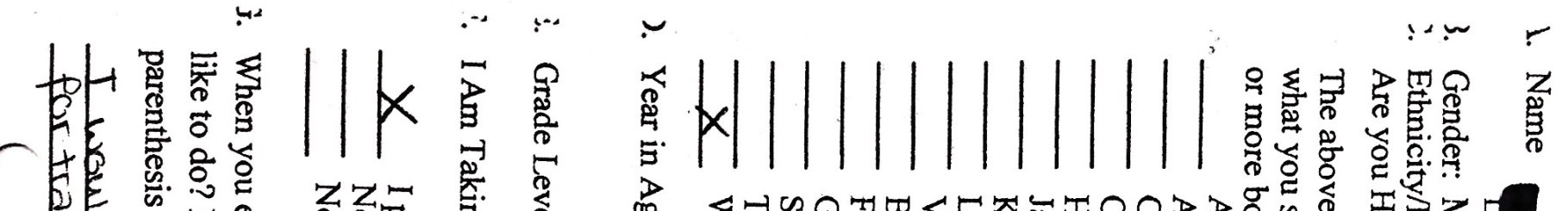

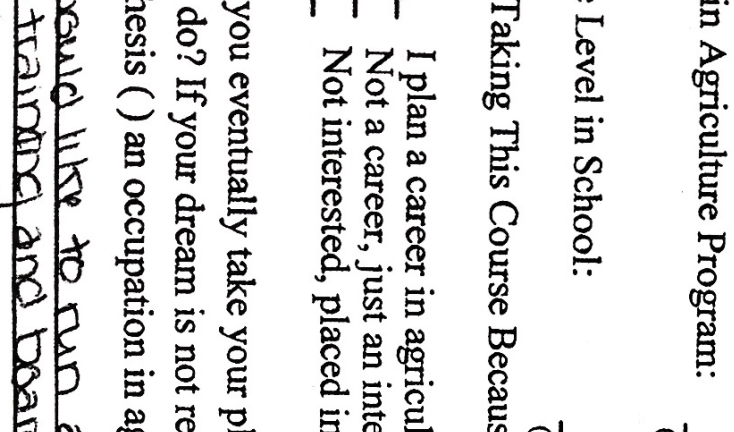

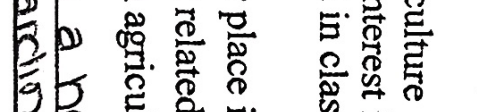

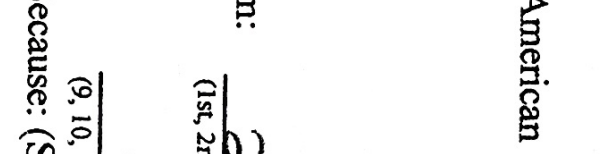

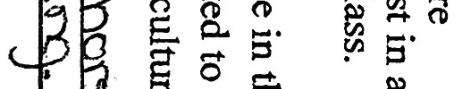

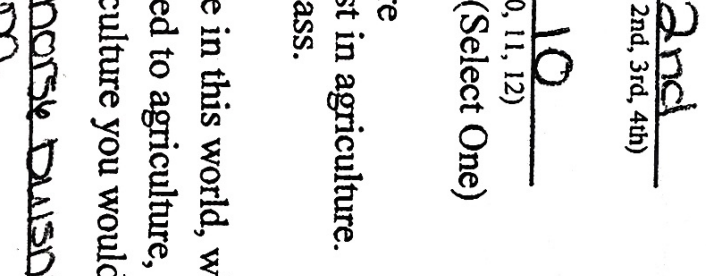

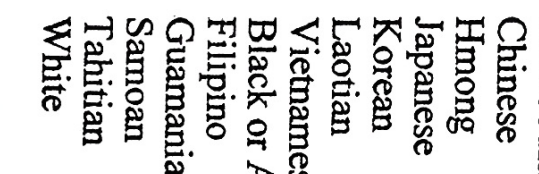

屏

窟高

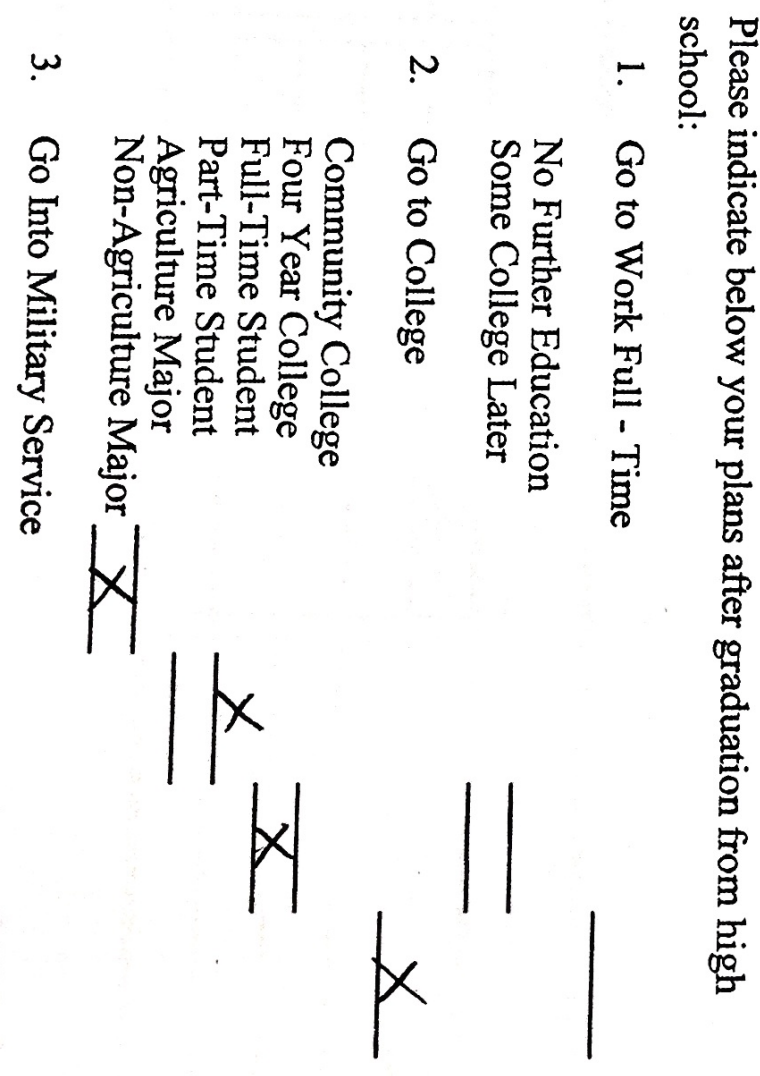

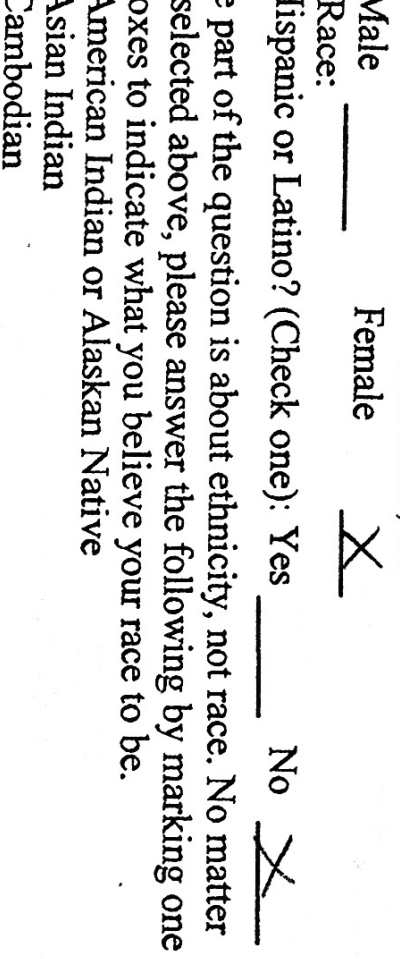

最

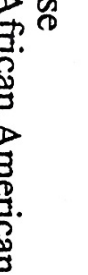

X

\section{|}

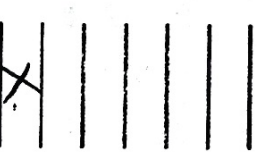

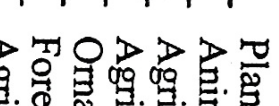

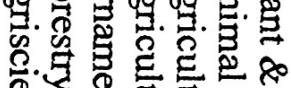

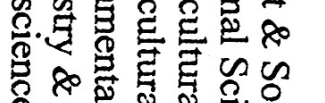

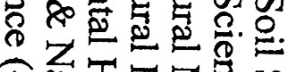

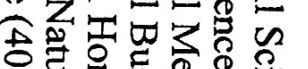

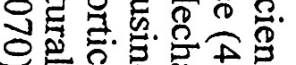

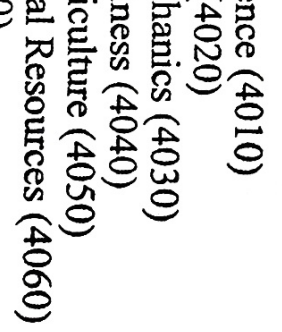

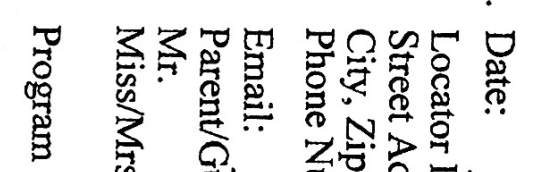

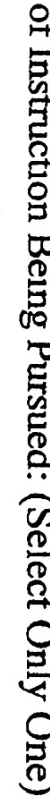

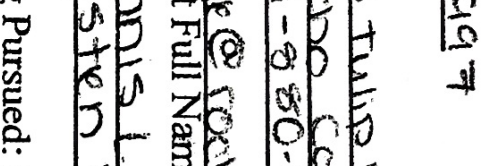
क्षिएक

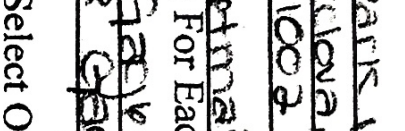

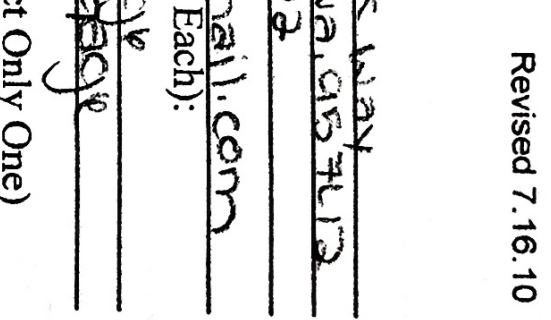




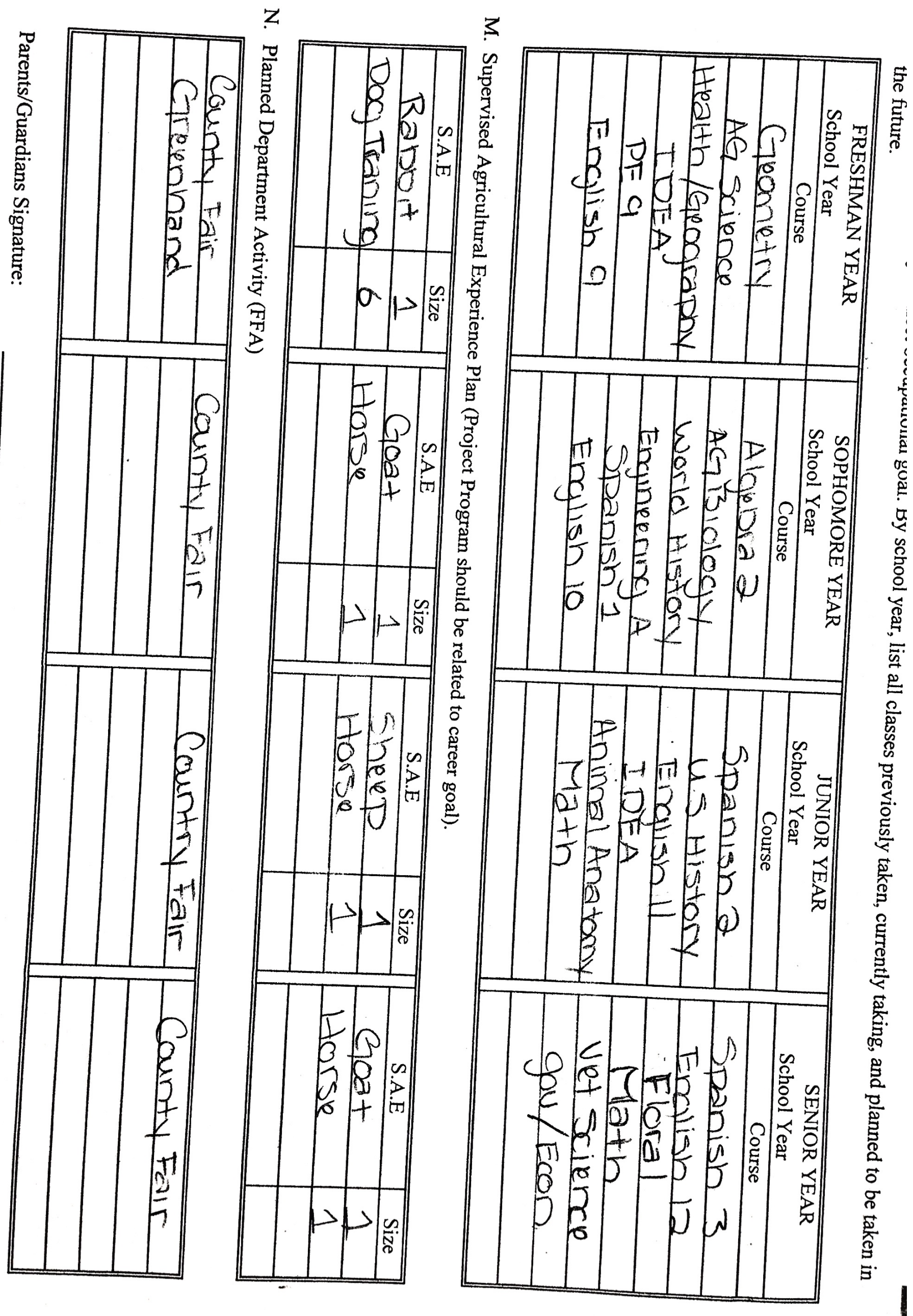




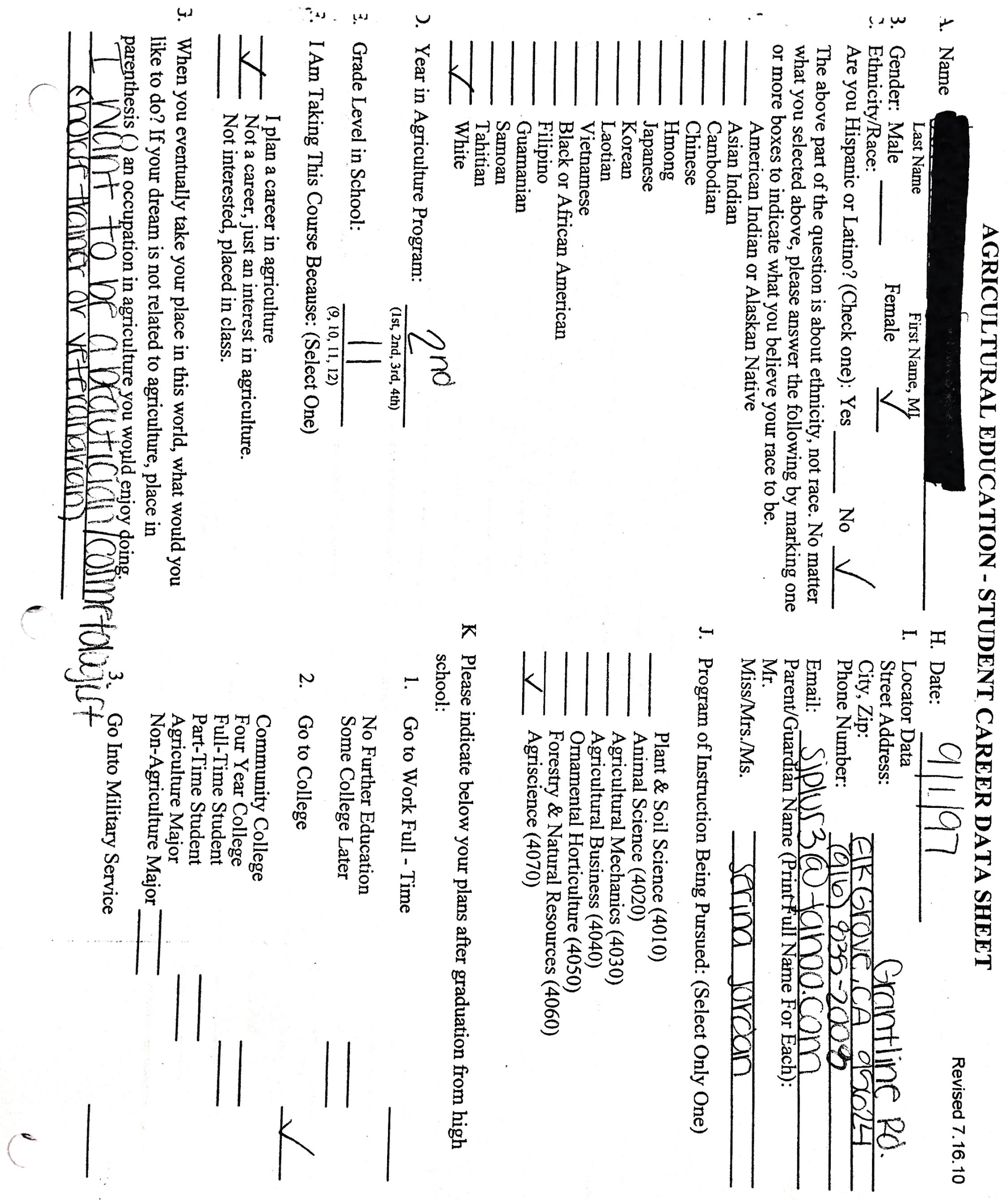




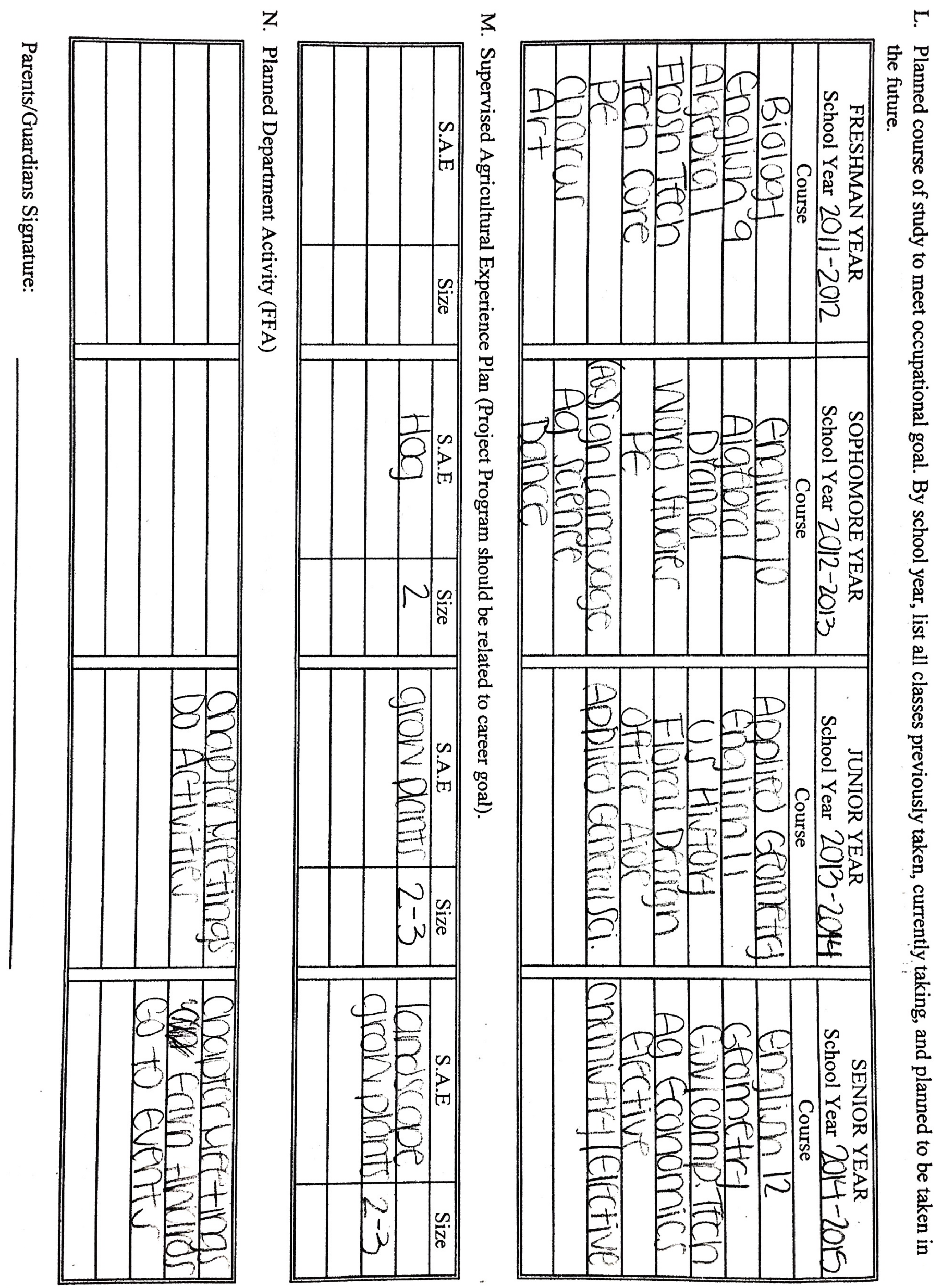


Support Material 2:

c Permanent Student File 


\section{Pleasant Grove High School Agriculture Department}

\section{Support Material 2: Permanent Student File}

Permanent student files are kept in the agriculture department office in the agriculture building. When a new student enters the program at the beginning of the year, a file is created for that students, where information is kept and can be accessed by all agriculture instructors.

Files are organized by graduating class year, and by last name. Any paper Record Books are kept a classroom file in the classroom students are in for that school year to ensure easy access for montly Record Book updates. Upon graduation, Record Books are moved to students' permanent files, and maintained for three years.

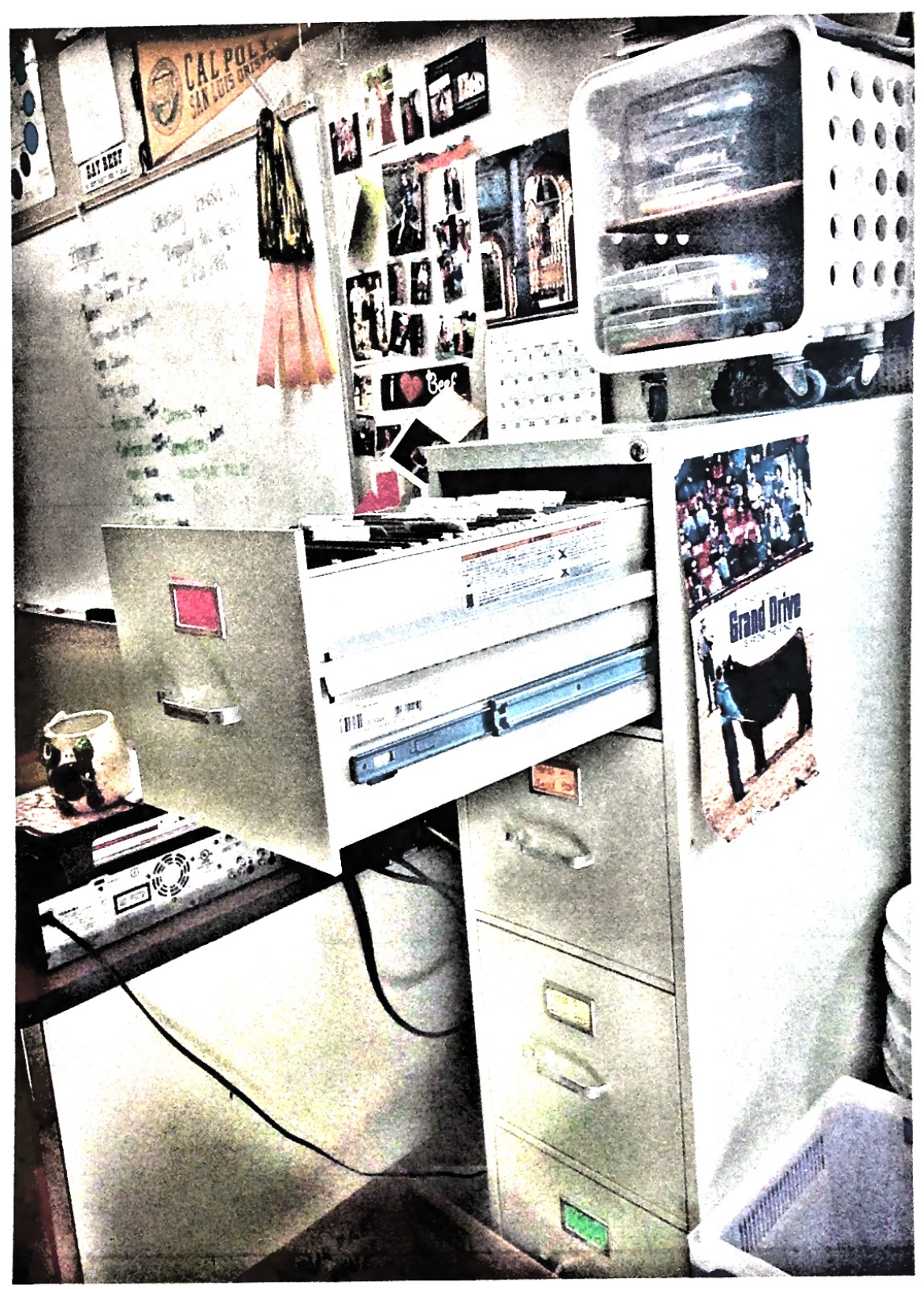


Support Material 3:

Agriculture Course Outlines 


\section{Pleasant Grove High School Agriculture Department}

\section{Support Material 3: Agriculture Course Outlines}

Course outlines are located in an electronic file within our school's internal server. The descriptions are also on file with the PGHS Counseling Department, as well as in our district's Comprehensive High School Course Catalog, available at the Elk Grove Unified School District's webpage. Most of these are older and have not been changed much since they were originally adopted. Attached are the course descriptions for all agriculture courses that I teach at Pleasant Grove High School:

Applied General Science

Elements \& Principles of Floral Design 
Applied General Science:

\begin{tabular}{|c|c|c|c|}
\hline Course 12203 & & & \\
\hline $\begin{array}{l}\text { Applied General } \\
\text { Science } \\
\text { "a-g"/m" approved } \\
\text { Florin High } \\
\text { Franklin High } \\
\text { Pleasant Grove High } \\
\text { Sheldon High }\end{array}$ & None & 10 & $\begin{array}{l}\text { This course explores earth science. chemistry. forces, work. energy. } \\
\text { waves. alternative energy sources, and nuclear energy as it pertains to } \\
\text { agriculture. Students are expected to function in both lab and lecture } \\
\text { situations and to work on basic equations. Students will be exposed to } \\
\text { the FFA. supervised occupational experience programs. and careers. } \\
\text { Homework consisting of reading. writing. lab reports. etc. will be } \\
\text { assigned. This course is part of a series of courses to prepare the } \\
\text { student for college level entry into the various disciplines of agricultural } \\
\text { science. This course meets UC and CSU elective requirements. This } \\
\text { course meets the General Science graduation requirement. This course } \\
\text { meets the Technology Proficiency Graduation Requirement at Florin } \\
\text { High Schood only. } \\
\text { Adopted curricular materials: Earth Science, Pearson }\end{array}$ \\
\hline Course 12206 & & & Adopted curricular materials \\
\hline
\end{tabular}

Elements \& Principles of Floral Design:

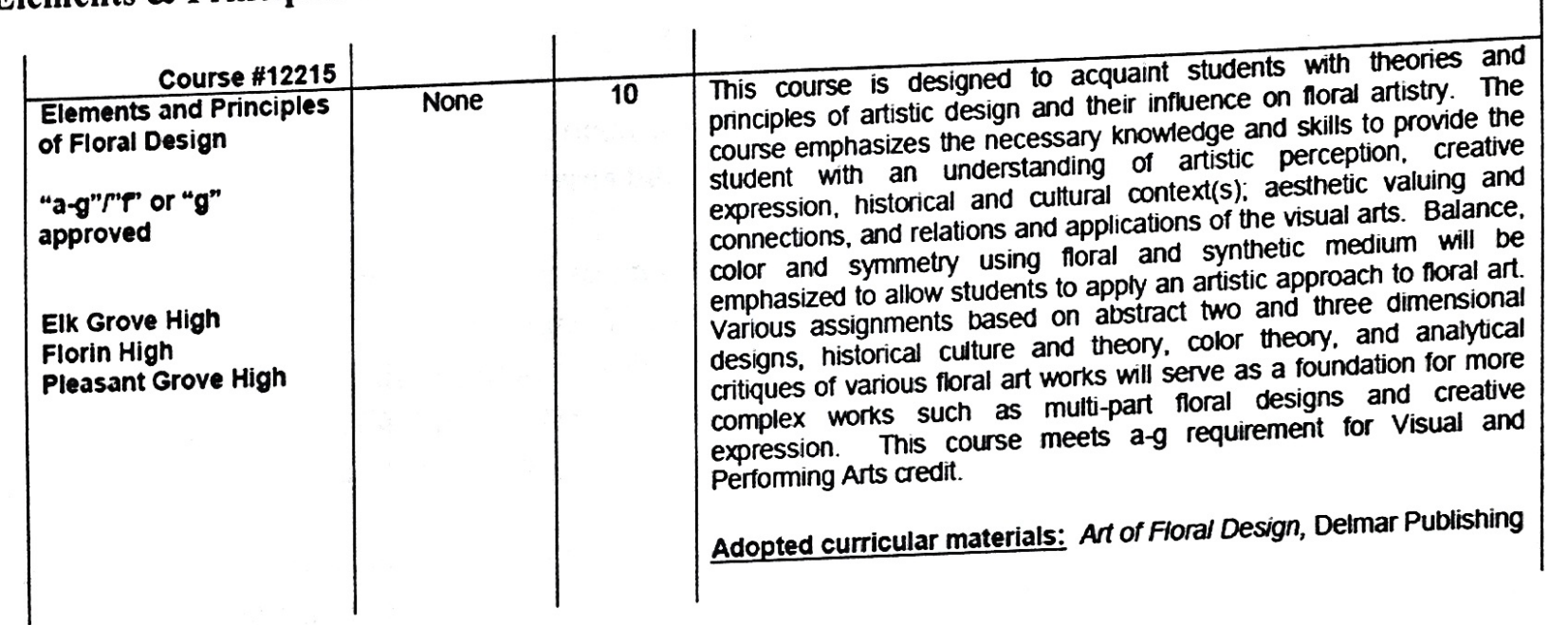




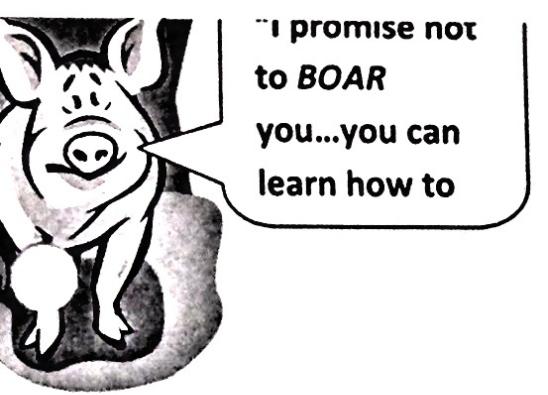

\title{
Pleasant Grove High School
}

\author{
Agriculture Department
}

Course Expectations 2013-2014

Instructor: Ms. Forster

Office Phone: 916-686-0230, ext, 4933

Email: jforster@egusd.net

\section{Course Description: Applied General Science}

This course will cover units such as Astronomy, Earth's Interior, Oceans, Plate Tectonics, etc. Future Farmers of America (FFA) will also be an integral part of this course. Students will be introduced to the FFA Program, California Agriculture, Careers in Agriculture, and much more.

II. Class Policies:

1. Be in your seat and ready to work, within one minute of the bell ringing. If you're not, you are tardy.

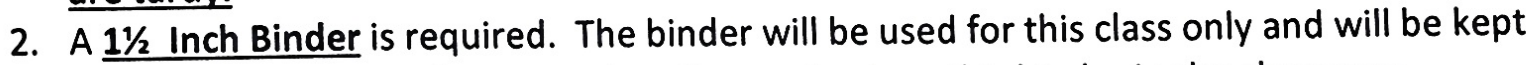
organized and neat. There is a place for you to store this binder in the classroom.

3. Each student must bring a pencil or pen to class everyday.

4. No food or drink is allowed in the classroom during class except water.

5. The classroom and Agriculture Department must be kept clean. All clean up activities will be done prior to students being dismissed.

III. Grading:

Your grade will be based on the following:

$50 \%$ Classroom work. This includes homework, in class assignments etc...

30\% Quizzes/Exams. This includes both semester finals.

$10 \%$ FFA Participation

$10 \%$ Supervised Agricultural Experience Project (SAE)

Letter grades will be assigned using the following guidelines:

$\begin{array}{llll}94 \% \text { and above } & \text { A } & 67-69 \% & \text { D+ } \\ 90-93 \% & \text { A- } & 64-66 \% & \text { D } \\ 87-89 \% & \text { B+ } & 60-63 \% & \text { D- } \\ 84-86 \% & \text { B } & 59 \% \text { and below } & \text { F } \\ 80-83 \% & \text { B- } & & \\ 77-79 \% & \text { C+ } & & \\ 74-76 \% & \text { C } & & \\ 70-73 \% & \text { C- } & & \end{array}$

\section{Note: GRADES WILL BE UPDATED ON TUESDAYS!!!}

IV. Homework and Class Assignments, $50 \%$ of grade

1. Late assignments will not be accepted (except in emergency situations).

2. In the event that homework is not turned in, students will complete a log in the "No Homework Binder" to document this occurrence.

3. Assignments should be neat and complete.

4. For each day you are absent, you have that many days to make it up.

5. Most weekly homework will be assigned Monday of each week.

V. Tests, $30 \%$ of grade

1. Tests will be given at the completion of each unit of study.

2. Quizzes may be given periodically as needed. 
3. There will be a Final Exam at the end of each semester.

4. Make up tests are the responsibility of the student! If you have an excused absence on the day of a test, it is your responsibility to make an appointment to take it within 1 week of returning to school - if a test is not made up within one week of the test being administered, a score of zero will be given for that test grade.

5. A student may re-take a previously completed test to attempt to earn a higher grade at any time before the current quarter ends by scheduling an appointment with the instructor for before or after school or during their lunch hour.

VI. SAE Projects $-10 \%$ of grade

1. All students are required to have a project that relates to a career in agriculture and requires 3 hours of labor per month.

2. Projects require prior approval of the instructor.

3. This project is $10 \%$ of your grade. If you have more than one $\mathrm{Ag}$ class, you only have to do one SAE project.

VII. FFA $-10 \%$ of grade

1. Your enrollment in this class automatically makes you an FFA member however, your level of participation depends on what you get interested in and your desire to get involved with all the various activities offered. If you are a $4-\mathrm{H}$ member you can definitely be active in both organizations and get a great experience from them!

2. The FFA is an integral part of this class therefore you are required to participate in various activities throughout the semester. FFA is the club within the Agriculture Department.

3. You are required to get $\mathbf{3}$ FFA credits per quarter and you will usually have more than $\mathbf{1 5}$ opportunities to do this. This should be the easiest part of your grade!

4. Examples of things you can do to get FFA Credits: Attend the once a month Chapter meeting put on by the Officer Team, participate in leadership conferences, public speaking contests, assist with fundraisers such as our Car Wash and much more! There are plenty of opportunities to get these credits!

VIII. Student Expectations

- Responsibility: You are expected to keep track and turn in your assignments on due dates. Excuses and whining are not permitted.

- Respect: All students have the right to learn and achieve without the interference of others.

Students will respect their property and that of others.

Exhibiting prejudice or prejudicial behaviors will NOT be tolerated.

$\sigma$ Students are expected to use acceptable and appropriate language. Profanity $\neq$ Respect

$\rightarrow$ Interrupting the teacher or another student who is speaking is rude and limits the opportunity for others to learn.

- Classroom Rules: No food or gum in the classroom. Water is allowed in re-closable containers. No hats or sunglasses are to be worn in the classroom. No electronic devices are to be used in the classroom. PGHS dress code will be followed daily.

- Be Prepared: Bring class binder, papers, pens/pencils, and your brain! (;)

\section{Consequences}

Students that REFUSE to follow the above behavioral expectations will be: 
1. Reminded of the rules and given a choice to abide by rules and told of consequences if reoccurs.

2. Continued disruptions, will result in an appropriate consequence (parent contact, detention, removal from class, referrals, etc.)

3. If a substitute teacher believes a student has refused to abide by the class rules, the student is required to complete a Substitute Behavior Essay, which is a 100 point assignment.

VI. Miscellaneous

- Academic Code of Conduct will be enforced. Refer to Parent/Student Handbook.

- The instructor has the right to alter, add to, or delete any portion of this class policy with prior notification to the class.

- Papers turned in with NO NAME will not be graded! If an assignment is turned in without a name, students may use a Renaissance Pass or STAR Card to resubmit the assignment.

- Any correspondence (e.g. phone calls, emails, etc.) will be responded within 2 business days of receiving them. If I am out of town on a FFA field trip, response time may be longer.

\section{Applied General Science Ms. Forster}

I have read and understand all the policies and procedures contained herein. Please sign and return to your instructor.

Student Signature

Parent/Guardian Signature

\section{Date}

Date 


\section{Pleasant Grove High School}

Agriculture Department - Course Expectations 2013-2014

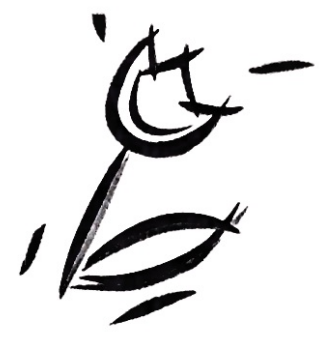

Instructor: Ms. Forster

Office Phone: 916-686-0230, ext., 4933

Email: jforster@egusd.net

IX. Course Description: Floral Design

This course will the basics of elements of floral design as well as basic elements of art history, the history of floral design, etc. FFA will also be an integral part of this course. Students will be compiling a professional portfolio of their designs and will also be exposed to California's agriculture and careers in the floral industry.

$X$. Class Policies:

1. Be in your seat and ready to work, within one minute of the bell ringing. If you're not, you are tardy.

2. A Spiral Bound Notebook is required. The binder will be used for this class only and will be kept organized and neat.

3. A three ring binder with a clear plastic cover will also be needed for your portfolio at the end of the school year.

4. Each student must bring a pencil or pen to class everyday.

5. No food or drink is allowed in the classroom during class except water.

6. The classroom and Agriculture Department must be kept clean. All cleanup activities will be done prior to students being dismissed.

7. Highly recommended:

- A USB flash drive to save your computer work on in the library.

XI. Grading:

Your grade will be based on the following:

$40 \%$ Classroom work. This includes homework, in class assignments etc...

25\% Projects (Wedding Project, Professional Portfolio, etc.)

15\% Quizzes/Exams

$10 \%$ FFA Participation

$10 \%$ Supervised Agricultural Experience Project (SAE)

Letter grades will be assigned using the following guidelines:

$\begin{array}{llrl}94 \% \text { and above } & \text { A } & 67-69 \% & \text { D+ } \\ 90-93 \% & \text { A- } & 64-66 \% & \text { D } \\ 87-89 \% & \text { B+ } & 60-63 \% & \text { D- } \\ 84-86 \% & \text { B } & 59 \% \text { and below } & \text { F } \\ 80-83 \% & \text { B- } & & \\ 77-79 \% & \text { C+ } & & \\ 74-76 \% & \text { C } & & \\ 70-73 \% & \text { C- } & & \end{array}$

Note: GRADES WILL BE UPDATED ON TUESDAYS!!!

XII. Homework and Class Assignments, $40 \%$ of grade

1. Late assignments will not be accepted (except in emergency situations).

2. Assignments should be neat and complete.

3. For each day you are absent, you have that many days to make it up. 
4. Most weekly homework will be assigned Monday of each week.

XIII. Projects, $25 \%$ of grade

1. All in class and assigned projects will count in this category of the grade - they can range from your professional portfolio to group presentations.

2. Students will receive detailed instruction on all projects at least two weeks prior to final due dates.

XIV. Quizzes/Exams, $15 \%$ of grade

1. Tests will be given at the completion of each unit of study.

2. Quizzes will be given periodically as needed.

3. Make up tests are the responsibility of the student! If you have an excused absence on the day of a test, it is your responsibility to make an appointment to take it within 1 week of returning to school - if a test is not made up within one week of the test being administered, a score of zero will be given for that test grade.

XV. SAE Projects $-10 \%$ of grade

1. All students are required to have a project that relates to a career in agriculture and requires 3 hours of labor per month.

2. Projects require prior approval of the instructor.

3. This project is $10 \%$ of your grade. If you have more than one Ag class, you only have to do one SAE project.

XVI. FFA $-10 \%$ of grade

1. Your enrollment in this class automatically makes you an FFA member however, your level of participation depends on what you get interested in and your desire to get involved with all the various activities offered. If you are a $4-\mathrm{H}$ member you can definitely be active in both organizations and get a great experience from them!

2. The FFA is an integral part of this class therefore you are required to participate in various activities throughout the semester. FFA is the club within the Agriculture Department.

3. You are required to get $\mathbf{3}$ FFA credits per quarter and you will usually have more than $\mathbf{1 5}$ opportunities to do this. This should be the easiest part of your grade!

4. Examples of things you can do to get FFA Credits: Attend the once a month Chapter meeting put on by the Officer Team, participate in leadership conferences, public speaking contests, assist with fundraisers such as our Car Wash and much more! There are plenty of opportunities to get these credits!

\section{Student Expectations}

- Responsibility: You are expected to keep track and turn in your assignments on due dates. Excuses and whining are not permitted.

- Respect: All students have the right to learn and achieve without the interference of others.

$\infty$ Students will respect their property and that of others.

Exhibiting prejudice or prejudicial behaviors will NOT be tolerated.

$\sigma$ Students are expected to use acceptable and appropriate language. Profanity $\neq$ Respect

Interrupting the teacher or another student who is speaking is rude and limits the opportunity for others to learn. 
- Classroom Rules: No food or gum in the classroom. Water is allowed in re-closable containers. No hats or sunglasses are to be worn in the classroom. No electronic devices are to be used in the classroom. PGHS dress code will be followed daily.

- Be Prepared: Bring class binder, papers, pens/pencils, and your brain! (-)

V. Consequences

Students that REFUSE to follow the above behavioral expectations will be:

4. Reminded of the rules and given a choice to abide by rules and told of consequences if reoccurs.

5. Continued disruptions, will result in an appropriate consequence (parent contact, detention, removal from class, referrals, etc.)

6. If a substitute teacher believes a student has refused to abide by the class rules, the student is required to complete a Substitute Behavior Essay.

VI. Miscellaneous

- Academic Code of Conduct will be enforced. Refer to Parent/Student Handbook.

- The instructor has the right to alter, add to, or delete any portion of this class policy with prior notification to the class.

- Papers turned in with NO NAME will not be graded!

- Students can resubmit a paper that previously did not have a name on it with a

Renaissance Pass or STAR Card for full credit within one week of the due date.

- Any correspondence (e.g. phone calls, emails, etc.) will be responded within 2 business days of receiving them. If I am out of town on a FFA field trip, response time may be longer.

\section{Floral Design Ms. Forster}

have read and understand all the policies and procedures contained herein. Please sign and return your instructor.

Student Signature

Parent/Guardian Signature
Date

Date 


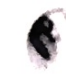

Support Material 4:

6

Course Gradebooks 


\section{Pleasant Grove High School Agriculture Department}

\section{Support Material 4: Course Gradebooks}

Gradeds are entered into an online system called SchoolLoop. Students and parents can view both grades and assigned work twenty four hours a day by accessing thei $r$ SchoolLoop Portal through our school home page. This year, a SchoolLoop App was also created for smart phone users to ensure easier access. Since all grades are kept online, there is no need for daily grade or attendance sheets or print outs, but students may request a print out of missing assingment from me at any time. 
Printed: 5/30/14

Forster, Jessica

Applied General Science P - Period 1

R pplied

Student ID:Grade\% Points

$99665489 \mathrm{~B}+$

99665891 A

\begin{tabular}{lll}
99667274 & $B+$ \\
\hline
\end{tabular}

99667406 A.

99667425

99668835 A

99668860

99669163 F

99669279 B.

99669472 F

99669543 B

99672900 B

$99675911 \mathrm{D}+$

99676990 C+

99684276

99693360 B-

99693700 B+

99695734 B

99699102 C-

99704499 c

99707194 A-

99708219 D

99728436 F

99731038 A

99732621

do9 99743208

077750515 C.

99764209 A

99766622 B-

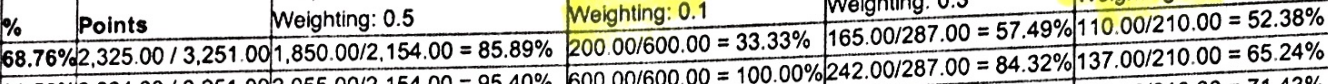

Pleasant Grove High School

Average: $74.37 \%$

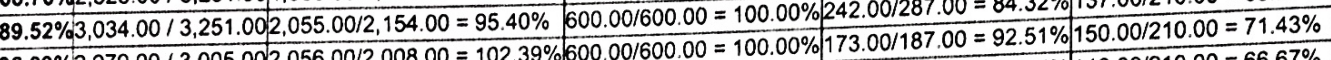

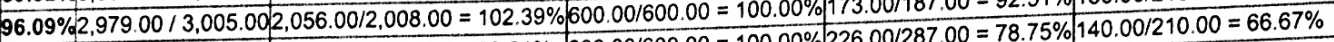

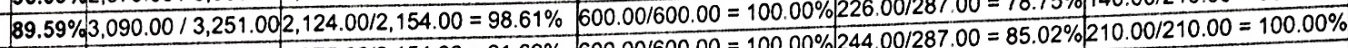

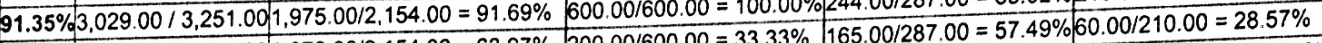

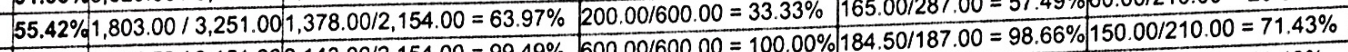

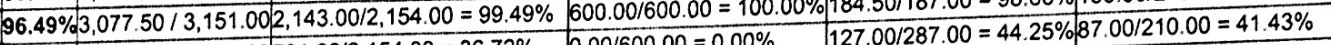
\begin{tabular}{|l|l|l|l|l|}
$35.78 \% 1,005.00 / 3,251.00791 .00 / 2,154.00=36.72 \%$ & $0.00 / 600.00=0.00 \%$ & $127.00 / 287.00$ & $44.25 \%$
\end{tabular} \begin{tabular}{ll|l|l|l|l|l|}
$57.56 \% 1,802.50 / 3,086.001,016.00 / 1,989.00=51.08 \%$ & $500.00 / 600.00=83.33 \%$ & $176.50 / 287.00=61.50 \%$ & $110.00 / 210.00=66.67 \%$
\end{tabular}

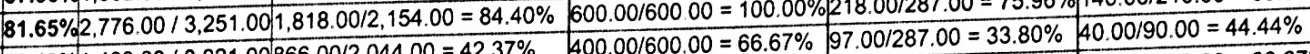

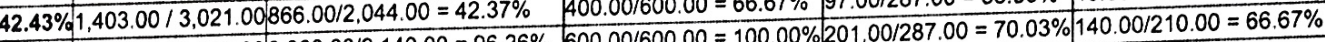

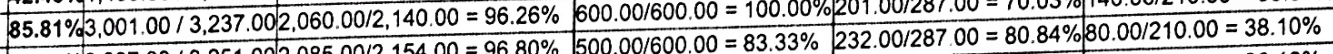

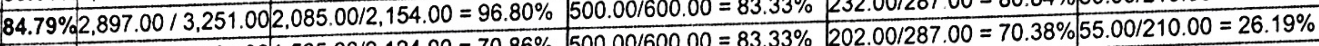
\begin{tabular}{|l|l|l|l|l|l|}
\hline $67.50 \% 2,262.00 / 3,221.00 / 1,505.00 / 2,124.00=70.86 \%$ & $500.00 / 600.00=83.33 \%$ & $202.00 / 287.00=70.38 \%$ & $55.00 / 210.00$ & $26.19 \%$ \\
\hline
\end{tabular}

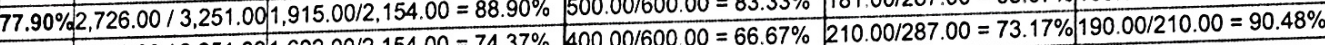

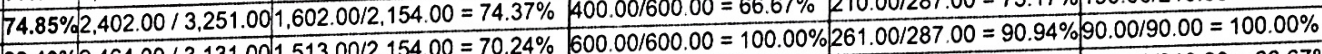

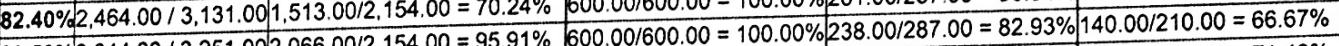

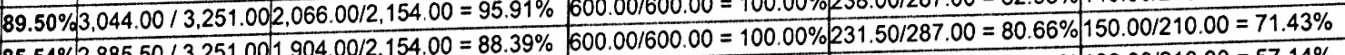

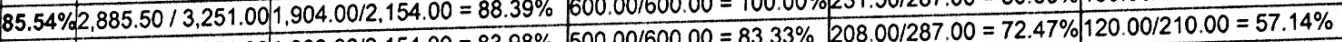

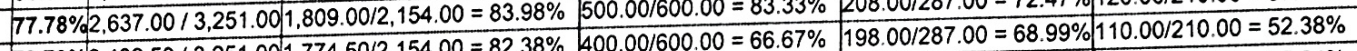

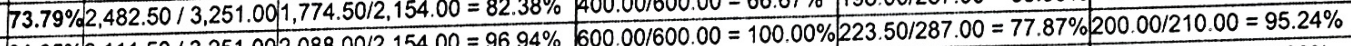
\begin{tabular}{l|l|l|l|l|}
$91.35 \% 3,111.50 / 3,251.002,088.00 / 2,154.00=96.94 \%$ & $600.00 / 600.00=100.00 \%$ & $160.50 / 287.00=55.92 \% 90.00 / 90.00=100.00 \%$
\end{tabular}

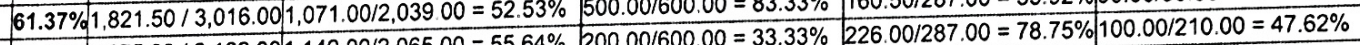
\begin{tabular}{ll|l|l|l|}
$59.54 \% 1,675.00 / 3,162.001 .149 .00 / 2,065.00$ & $=00 / 210.00=95.24 \%$
\end{tabular}

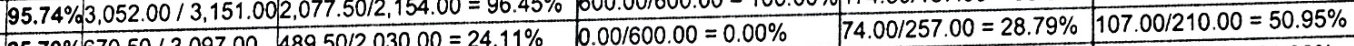
\begin{tabular}{|l|l|l|l|l|}
$25.79 \% 670.50 / 3.097 .00$ & $489.50 / 2,030.00=24.11 \%$ & $0.00 / 600.00$ & $0.001287 .00=51.22 \% 67.00 / 210.00=31.90 \%$
\end{tabular}

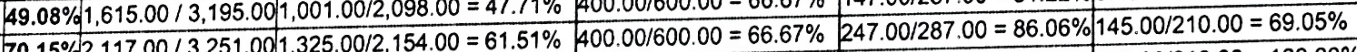

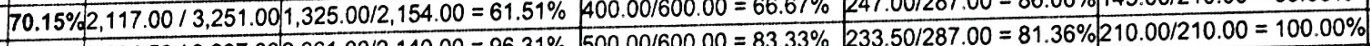

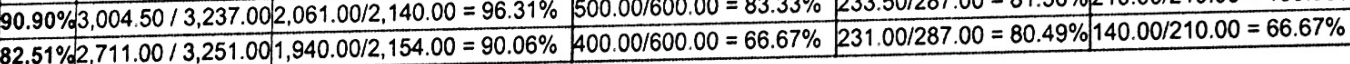


Forster, Jessica

Pleasant Grove High School

Applied General Science P. Period 1

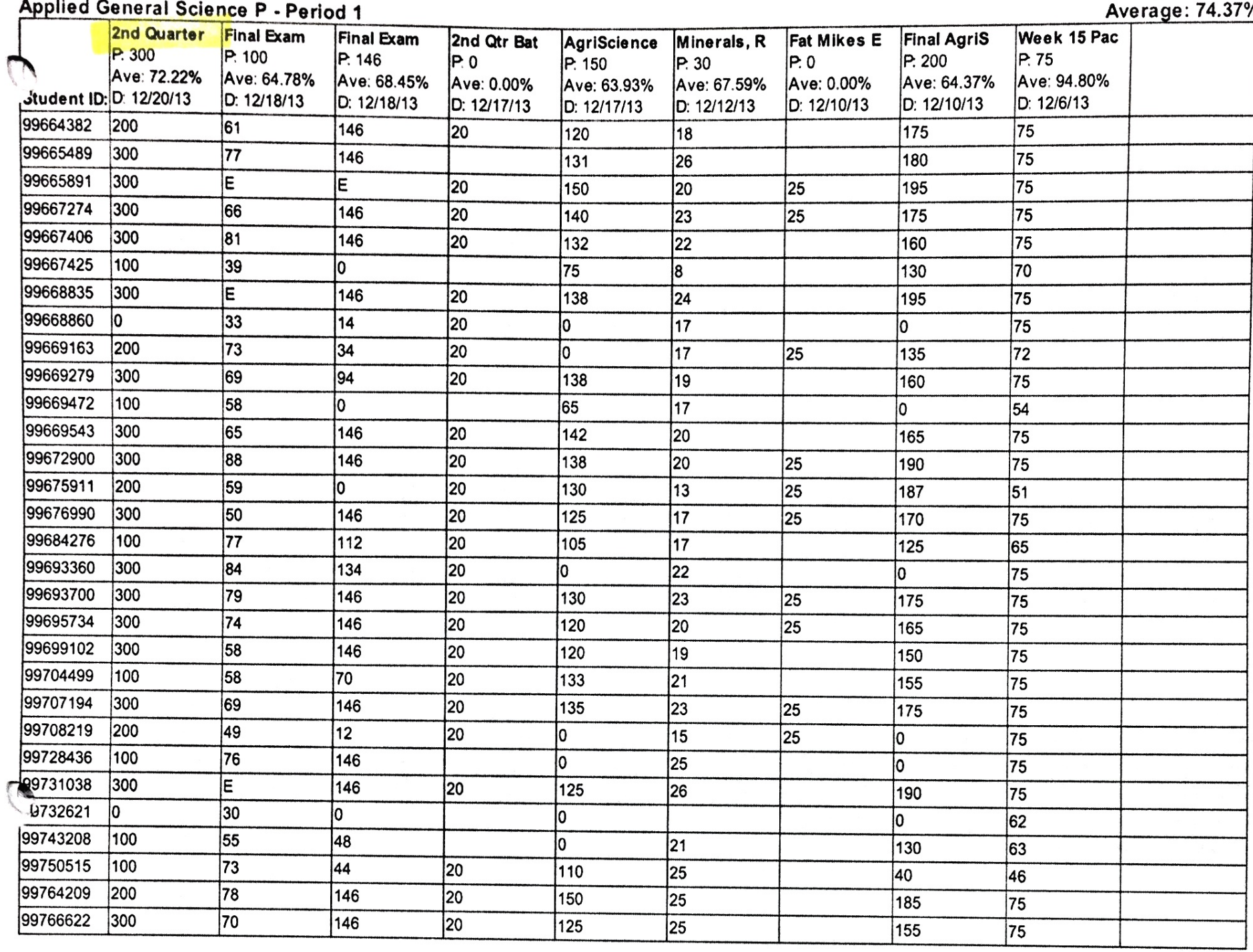


Forster, Jessica

Applied General Science P. Period 1

Pleasant Grove High School

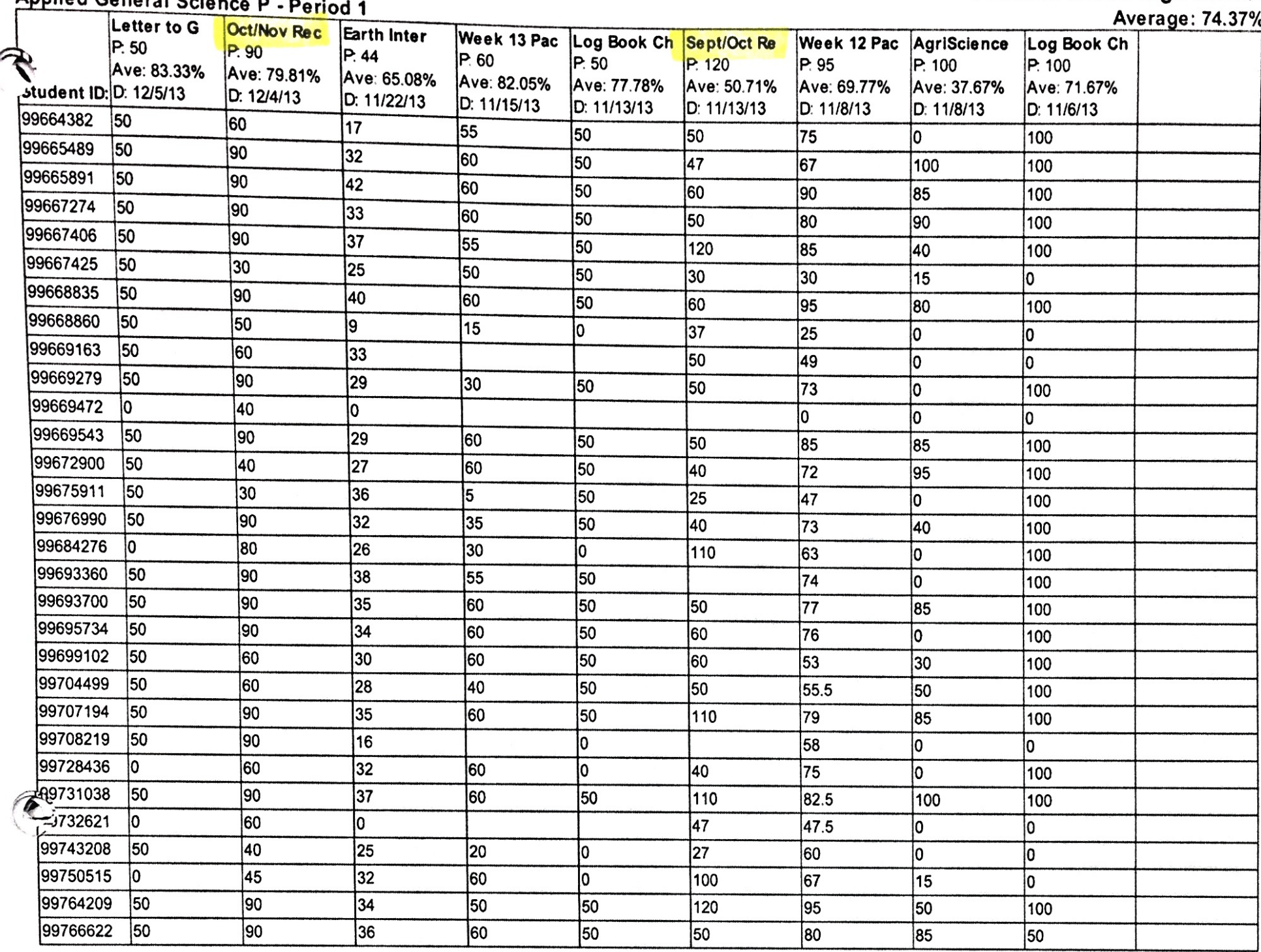


Forster, Jessica

Applied General Science P-Period 1

Pleasant Grove High School

\begin{tabular}{|c|c|c|c|c|c|c|c|c|c|c|}
\hline student ID: & $\begin{array}{l}\text { Week } 11 \text { Pac } \\
\text { P. } 10 \\
\text { Ave: } 75.33 \% \\
\text { D. } 11 / 4 / 13 \\
\end{array}$ & $\begin{array}{l}\text { Weok } 10 \text { Pac } \\
\text { P: } 85 \\
\text { Ave: } 75.02 \% \\
\text { D: } 10 / 25 / 13 \\
\end{array}$ & $\begin{array}{l}\text { Astronomy U } \\
\text { P. } 53 \\
\text { Ave: } 67.99 \% \\
\text { D: } 10 / 22 / 13\end{array}$ & $\begin{array}{l}\text { Solar Syste } \\
\text { P. } 77 \\
\text { Ave: } 58.10 \% \\
\text { D: } 10 / 22 / 13\end{array}$ & $\begin{array}{l}\text { Week } 9 \text { Pack } \\
\text { P. } 40 \\
\text { Ave: } 88.58 \% \\
\text { D: } 10 / 18 / 13\end{array}$ & $\begin{array}{l}\text { Astronomy R } \\
\text { P. } 0 \\
\text { Ave: } 0.00 \% \\
\text { D: } 10 / 21 / 13\end{array}$ & $\begin{array}{l}\text { Astronomy S } \\
\text { P. } 31 \\
\text { Ave: } 64.63 \% \\
\text { D: } 10 / 21 / 13\end{array}$ & $\begin{array}{l}\text { Revised Met } \\
\text { P. } 25 \\
\text { Ave: } 62.67 \% \\
\text { D: } 10 / 18 / 13 \\
\end{array}$ & $\begin{array}{l}\text { 1st Qtr Bat } \\
\text { P: } 0 \\
\text { Ave: } 0.00 \% \\
\text { D: } 10 / 11 / 13 \\
\end{array}$ & \\
\hline 99664382 & 10 & 70 & 27 & 57 & 40 & 4 & 16 & 25 & 20 & \\
\hline 99665489 & 10 & 77 & 46 & 71 & 39 & 10 & 29 & 25 & & \\
\hline 99665891 & 10 & 77 & 41 & 85 & 38 & 10 & 31 & 25 & 20 & \\
\hline 99667274 & 10 & 76 & 42 & 65 & 40 & 10 & 31 & 25 & 20 & \\
\hline 99667406 & 10 & 69 & 45 & 75 & 40 & 10 & 29 & 25 & & \\
\hline 99667425 & 10 & 58 & 48 & 28 & 35 & 10 & 0 & 25 & 20 & \\
\hline 99668835 & 10 & 75 & 50 & 76 & 40 & 8.5 & 31 & 25 & 20 & \\
\hline 99668860 & 1 & 48 & 30 & 0 & 34 & 5 & 0 & 0 & 20 & \\
\hline 99669163 & 1 & 63 & 29 & 7 & 29 & 8.5 & 5 & 0 & & \\
\hline 99669279 & 10 & 69 & 41 & 67 & 40 & 10 & 14 & 25 & 20 & \\
\hline 99669472 & 0 & 0 & 0 & 0 & 0 & 10 & 0 & 0 & 10 & \\
\hline 99669543 & 10 & 70 & 35 & 67 & 37 & 10 & 31 & 25 & 20 & \\
\hline 99672900 & 10 & 74 & 39 & 68 & 40 & 10 & 29 & 0 & 20 & \\
\hline 99675911 & 1 & 46 & 47 & 64 & 27 & 5 & 0 & 0 & 20 & \\
\hline 99676990 & 10 & 72 & 31 & 28 & 40 & 10 & 31 & 25 & 10 & \\
\hline 99684276 & 10 & 72 & 36 & 60 & 40 & 10 & 4 & 25 & 10 & \\
\hline 99693360 & 10 & 63 & 43 & 31 & 38 & 10 & 17 & 0 & 20 & \\
\hline 99693700 & 10 & 77 & 37 & 65 & 40 & 10 & 31 & 25 & & \\
\hline 99695734 & 1 & 69 & 39 & 61 & 40 & 5.5 & 31 & 0 & 20 & \\
\hline 99699102 & 10 & 63 & 36 & 39 & 37 & 7 & 16 & 0 & 10 & \\
\hline 99704499 & 10 & 55 & 36 & 30 & 39 & 10 & 31 & 25 & 20 & \\
\hline 99707194 & 10 & 68 & 41 & 50 & 40 & 9.5 & 31 & 25 & 20 & \\
\hline 99708219 & 10 & 55 & 28 & 18 & 40 & 8.5 & 4 & 0 & 20 & \\
\hline 99728436 & 10 & 64 & 40 & 0 & 40 & 7 & 31 & 0 & 20 & \\
\hline f9731038 & 1 & 71 & 46 & 80 & 40 & 9.5 & 31 & 25 & 20 & \\
\hline$=-9732621$ & 10 & 50 & 19 & 0 & 33 & 4 & 0 & 0 & & \\
\hline 99743208 & 0 & 61 & 0 & 0 & 0 & $E$ & & 25 & 20 & \\
\hline 99750515 & 10 & 65 & 50 & 17 & 37 & 10 & 19 & 20 & 20 & \\
\hline 99764209 & 1 & 70 & 42 & 65 & 40 & 5.5 & 31 & 25 & 20 & \\
\hline 99766622 & 10 & 66 & 37 & 68 & 40 & 10 & 27 & 25 & & \\
\hline
\end{tabular}


Forster, Jessica

Pleasant Grove High School

Applied General Science P. Period 1

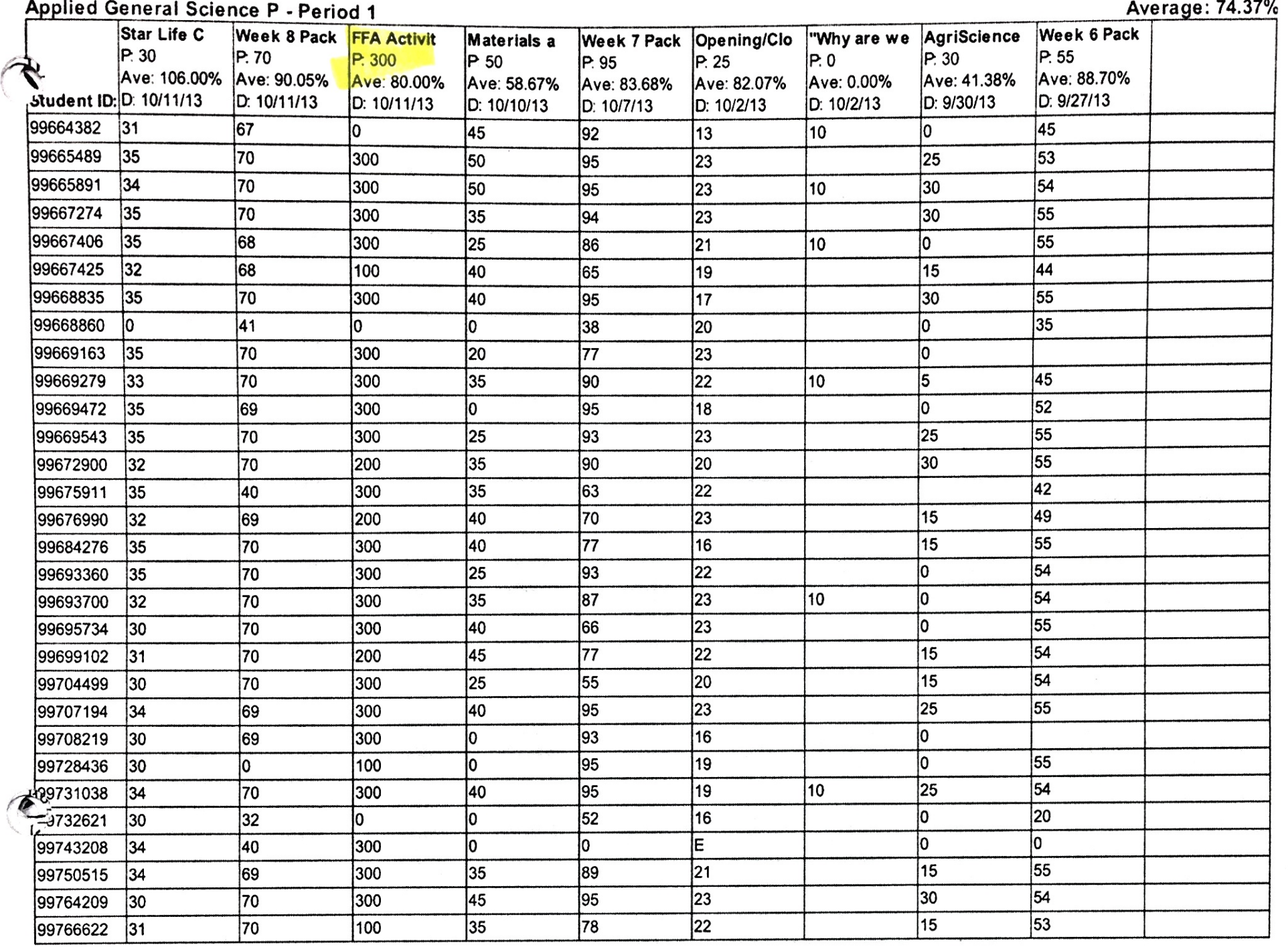


Forster, Jessica

Pleasant Grove High School

Applied General Science P - Period 1

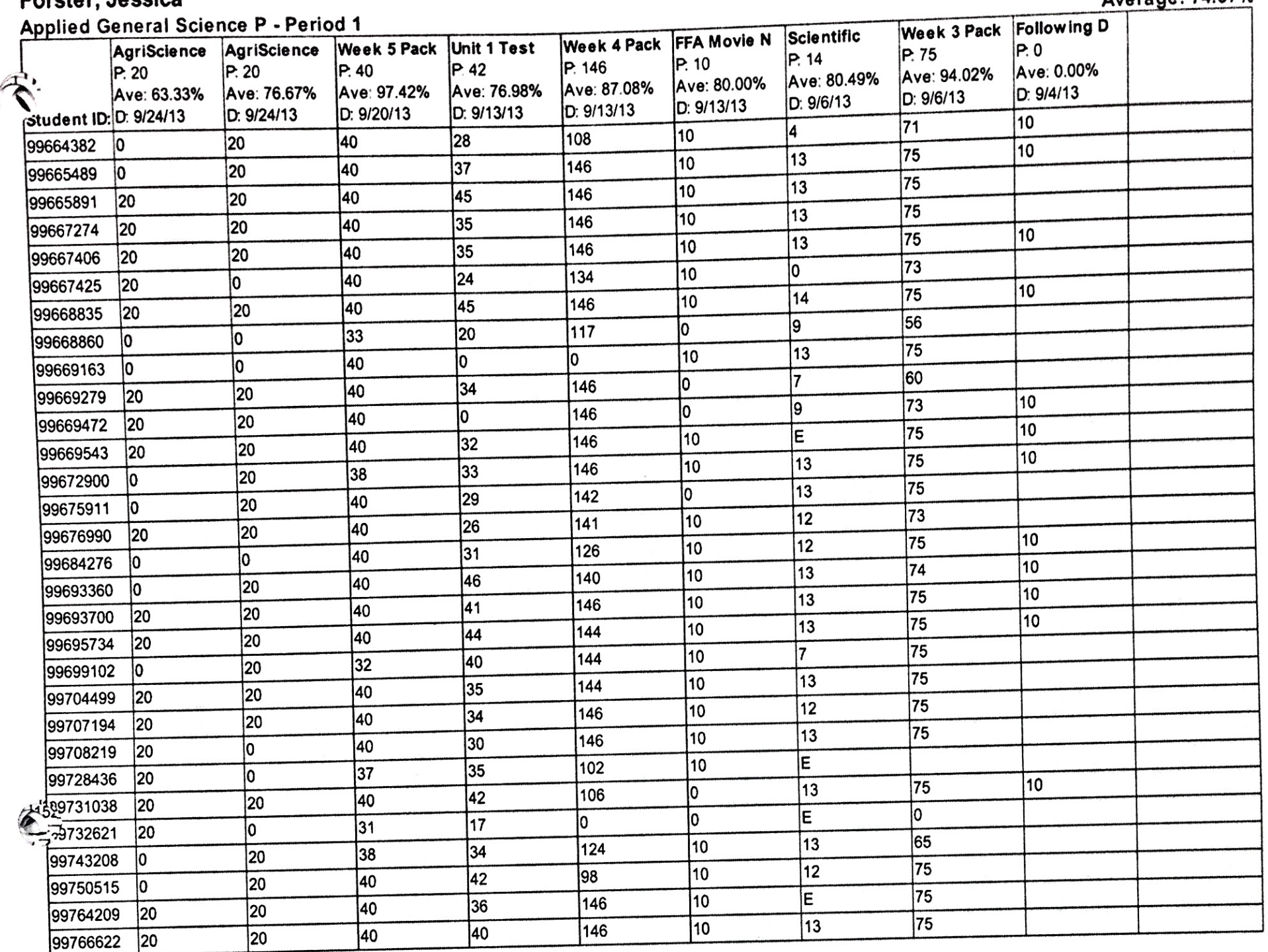


Forster, Jessica

Applied General Science P. Period 1

Pleasant Grove High School

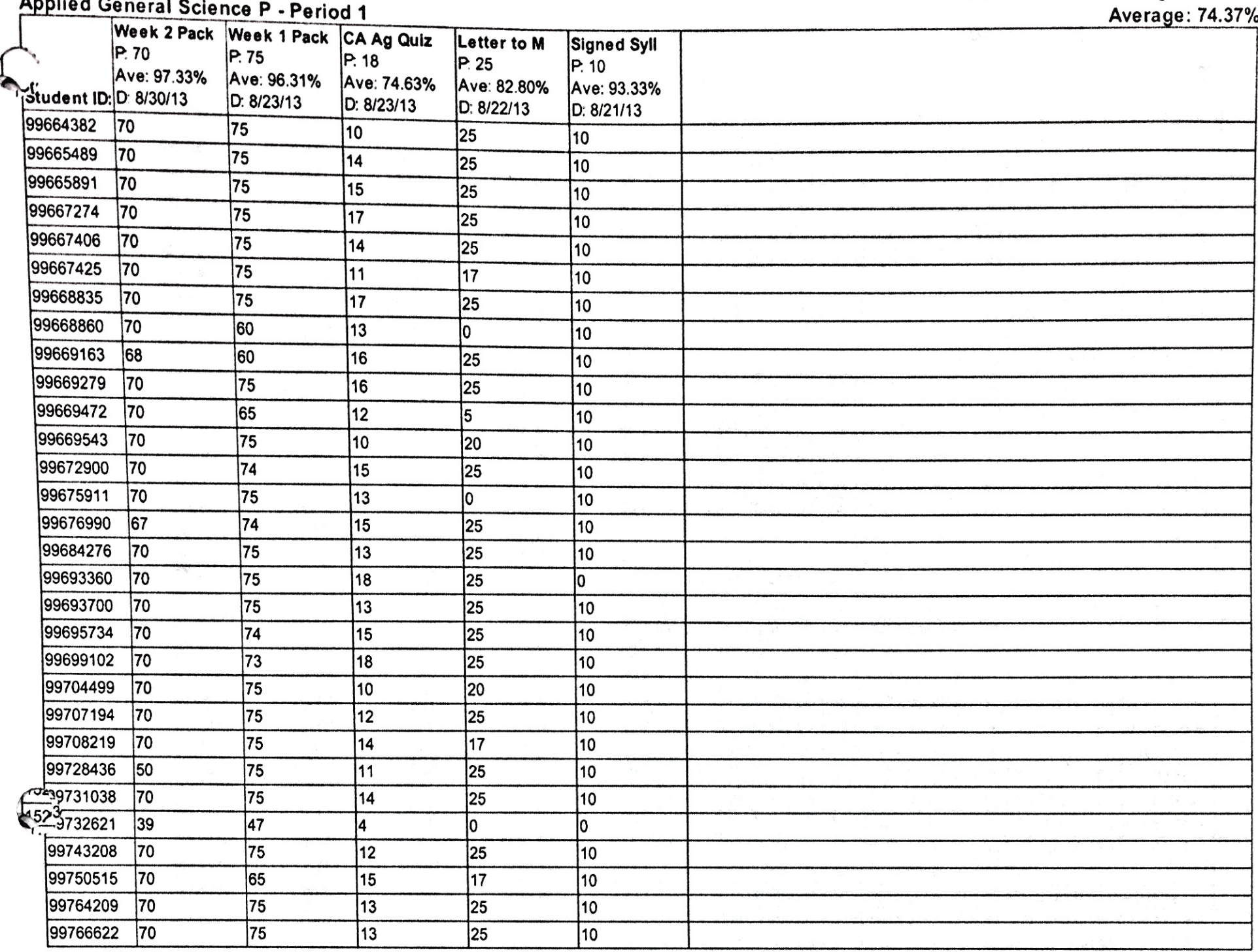


Forster, Jessica

The Elements \& Principles of Floral Design - Period 2

1.

Student ID Grade \%

Clas sroom

P. 867.00

Weighting: 0.4

FFA Participation
P. 600.00

P. 600.00
Weighting: 0.1
Pleasant Grove High School

Average: $84.47 \%$

\begin{tabular}{l|l|l|l|l|l|l|l|}
14020400 & $86.34 \% 1,938.00 / 2,146.00$ & $787.00 / 867.00=90.77 \%$ & $600.00 / 600.00=100.00 \% 348.00 / 400.00=87.00 \%$ & $73.00 / 99.00=73.74 \%$ & $130.00 / 180.00=72.22 \%$
\end{tabular}

14035000 A $95.30 \% 2,092.00 / 2,146.00879 .00 / 867.00=101.38 \% 600.00 / 600.00=100.00 \% 379.00 / 400.00=94.75 \% 84.00 / 99.00=84.85 \% 150.00 / 180.00=83.33 \%$

\begin{tabular}{|l|l|l|l|l|l|l|l|l|}
\hline 14035800 & B- & $80.64 \% 1,563.00 / 2,146.00807 .00 / 867.00=93.08 \%$ & $200.00 / 600.00=33.33 \%$ & $356.00 / 400.00=89.00 \%$ & $70.00 / 99.00=70.71 \%$ & $130.00 / 180.00=72.22 \%$
\end{tabular}

\begin{tabular}{|l|l|l|l|l|l|}
\hline 14063300 & B- & $82.97 \% 1,364.00 / 1,913.00611 .00 / 679.00=89.99 \%$ & $200.00 / 600.00=33.33 \%$ & $356.00 / 400.00=89.00 \% 47.00 / 54.00=87.04 \%$ & $150.00 / 180.00=83.33 \%$
\end{tabular}

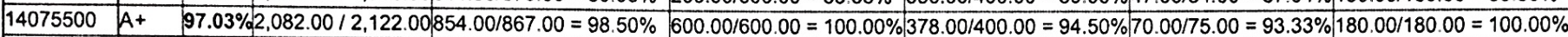

14109400 A. $\quad 92.48 \% 2,028.00 / 2,146.00 / 846.00 / 867.00=97.58 \% \quad 600.00 / 600.00=100.00 \% 357.00 / 400.00=89.25 \% 90.00 / 99.00=90.91 \% 135.00 / 180.00=75.00 \%$

\begin{tabular}{|l|l|l|l|l|l|l|l|}
\hline 14197900 & $\mathrm{~B}+$ & $\mathbf{8 7 . 8 8} \% 1,647.00 / 2,121.00814 .00 / 842.00=96.67 \%$ & $200.00 / 600.00=33.33 \%$ & $368.00 / 400.00=92.00 \% 85.00 / 99.00=85.86 \%$ & $180.00 / 180.00=100.00 \%$ \\
\hline
\end{tabular}

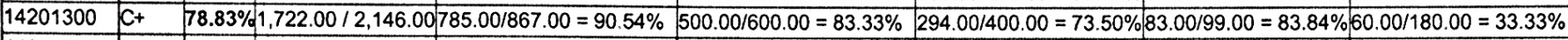

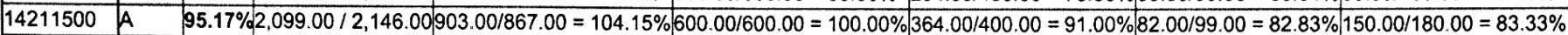

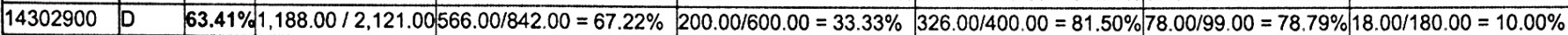

\begin{tabular}{|l|l|l|l|l|l|l|}
\hline 14319000 & C & $72.76 \% 1,288.00 / 2,146.00$ & $817.00 / 867.00=94.23 \%$ & $0.00 / 600.00=0.00 \%$ & $314.00 / 400.00=78.50 \% 70.00 / 99.00=70.71 \% 87.00 / 180.00=48.33 \%$ \\
\hline
\end{tabular}

\begin{tabular}{|l|l|l|l|l|l|}
\hline 14333600 & B & $83.80 \% 1,903.00 / 2,146.00$ & $822.00 / 867.00=94.81 \%$ & $600.00 / 600.00=100.00 \%$ & $310.00 / 400.00=77.50 \% 73.00 / 99.00=73.74 \% 98.00 / 180.00=54.44 \%$
\end{tabular}

\begin{tabular}{|l|l|l|l|l|l|l|}
\hline 14376600 & $\mathrm{~B}$ & $85.18 \% 1,650.00 / 1,936.00$ & $737.00 / 837.00=88.05 \%$ & $500.00 / 600.00=83.33 \%$ & $331.00 / 400.00=82.75 \%$ & $82.00 / 99.00=82.83 \%$ \\
\hline
\end{tabular}

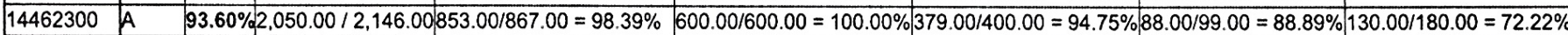

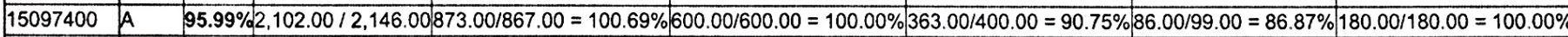

\begin{tabular}{|l|l|l|l|l|l|l|}
\hline 15103900 & B- & $80.24 \% 1.477 .00 / 2.146 .00816 .00 / 867.00=94.12 \%$ & $100.00 / 600.00=16.67 \%$ & $355.00 / 400.00=88.75 \%$ & $76.00 / 99.00=76.77 \%$ & $130.00 / 180.00=72.22 \%$
\end{tabular}

\begin{tabular}{|l|l|l|l|l|}
\hline 15132100 & $\mathrm{~B}+$ & $88.42 \% 2.014 .00 / 2.146 .00886 .00 / 867.00=102.19 \% 600.00 / 600.00=100.00 \% 326.00 / 400.00=81.50 \% 62.00 / 99.00=62.63 \%$ & $140.00 / 180.00=77.78 \%$
\end{tabular}

\begin{tabular}{|l|l|l|l|l|l|l}
\hline 15139500 & $\mathrm{C}+$ & $78.41 \% 1.636 .00 / 2,146.00$ & $748.00 / 867.00=86.27 \%$ & $400.00 / 600.00=66.67 \%$ & $352.00 / 400.00=88.00 \% 80.00 / 99.00=80.81 \%$ & $56.00 / 180.00=31.11 \%$
\end{tabular}

\begin{tabular}{|l|l|l|l|l|l|l|l|}
\hline 15225300 & D+ & $69.34 \% 1.510 .00 / 2,146.00728 .00 / 867.00=83.97 \%$ & $400.00 / 600.00=66.67 \%$ & $275.00 / 400.00=68.75 \% 62.00 / 99.00=62.63 \% 45.00 / 180.00=25.00 \%$ \\
\hline 152580
\end{tabular}

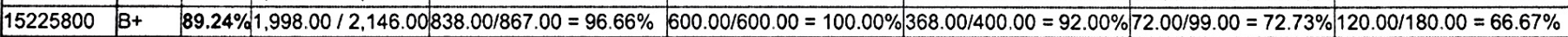

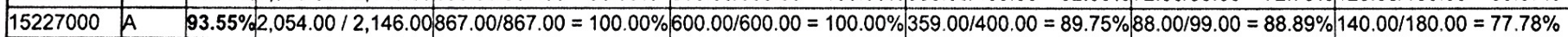

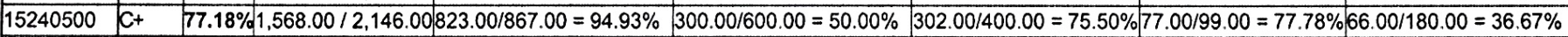

\begin{tabular}{l|l|l|l|l|}
15252700 & $B+$ & $87.24 \% 1,911.00 / 2,096.00724 .00 / 817.00=88.62 \%$ & $600.00 / 600.00=100.00 \% 341.00 / 400.00=85.25 \% 71.00 / 99.00=71.72 \%$ & $175.00 / 180.00=97.22 \%$
\end{tabular} 15256600 A- $\quad 91.48 \% 2,048.00 / 2,146.00890 .00 / 867.00=102.65 \% 600.00 / 600.00=100.00 \% 334.00 / 400.00=83.50 \% 74.00 / 99.00=74.75 \% \quad 150.00 / 180.00=83.33 \%$

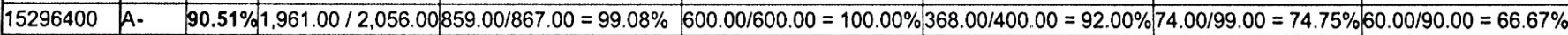
\begin{tabular}{|l|l|l|l|l|l|}
\hline 15345200 & $B$ & $86.50 \% 1,953.00 / 2,146.00837 .00 / 867.00=96.54 \%$ & $600.00 / 600.00=100.00 \% 277.00 / 400.00=69.25 \%$ & $76.00 / 99.00=76.77 \%$ & $163.00 / 180.00=90.56 \%$
\end{tabular}

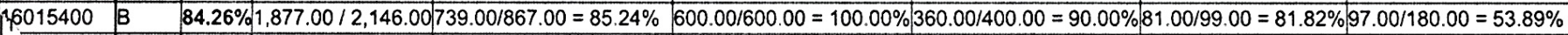
\begin{tabular}{|l|l|l|l|l|l|l|}
\hline 637600 & $C+$ & $77.12 \% 1,568.00 / 2,146.00857 .00 / 867.00=98.85 \%$ & $300.00 / 600.00=50.00 \%$ & $215.00 / 400.00=53.75 \% 86.00 / 99.00=86.87 \%$ & $110.00 / 180.00=61.11 \%$
\end{tabular} \begin{tabular}{|c|c|c|c|c|c|c|}
\hline 99658645 & $B+$ & $89.91 \%$ & $1,997.00 / 2,146.00864 .00 / 867.00=99.65 \%$ & $600.00 / 600.00=100.00 \% 328.00 / 400.00=82.00 \%$ & $85.00 / 99.00=85.86 \%$ & $120.00 / 180.00=66.67 \%$ \\
\hline
\end{tabular} \begin{tabular}{ll}
\hline 99663197 & $\mathrm{~B}+$
\end{tabular} \begin{tabular}{|l|l|l|l}
99675028 & $F$
\end{tabular} $89.62 \% 1,648.00 / 1,781.00602 .00 / 682.00=88.27 \% \quad 600.00 / 600.00=100.00 \% 362.00 / 400.00=90.50 \% 84.00 / 99.00=84.85 \%-$ \begin{tabular}{|l|l|l|l|l|l|l|l|}
\hline 99717071 & $B$ & $86.52 \% 1,527.00 / 1,936.00704 .00 / 702.00=100.28 \%$ & $300.00 / 600.00=50.00 \%$ & $338.00 / 400.00=84.50 \% 45.00 / 54.00=83.33 \%$ & $140.00 / 180.00=77.78 \%$ \\
\hline
\end{tabular}

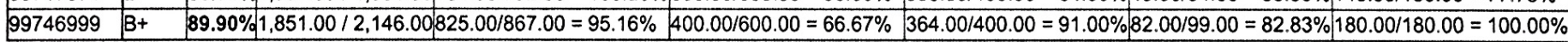


Pleasant Grove High School

Average: $84.47 \%$

Forster, Jessica

The Elements \& Principles of Floral Design - Period 2

\begin{tabular}{|c|c|c|c|c|c|c|c|c|c|c|}
\hline Ft' & $\begin{array}{l}\text { 2nd Quarter } \\
\text { P. } 300 \\
\text { Ave: } 70.71 \% \\
D: 12 / 20 / 13 \\
\end{array}$ & $\begin{array}{l}\text { History of } \\
\text { P. } 150 \\
\text { Ave: } 78.36 \% \\
\text { D: } 12 / 17 / 13 \\
\end{array}$ & $\begin{array}{l}\text { Fat Mikes E } \\
\text { P. } 0 \\
\text { Ave: } 0.00 \% \\
\text { D: } 12 / 10 / 13\end{array}$ & $\begin{array}{l}\text { Letter to } \mathbf{G} \\
\text { P. } 50 \\
\text { Ave: } 96.88 \% \\
\text { D: } 12 / 5 / 13\end{array}$ & $\begin{array}{l}\text { Oct/Nov Rec } \\
\text { P. } 90 \\
\text { Ave: } 73.44 \% \\
\text { D: } 12 / 4 / 13 \\
\end{array}$ & $\begin{array}{l}\text { December Re } \\
\text { P. } 45 \\
\text { Ave: } 81.95 \% \\
\text { D: } 12 / 3 / 13 \\
\end{array}$ & $\begin{array}{l}\text { November Re } \\
\text { P. } 45 \\
\text { Ave: } 74.95 \% \\
\text { D: } 11 / 19 / 13 \\
\end{array}$ & $\begin{array}{l}\text { Notebook Ch } \\
\text { P. } 55 \\
\text { Ave: } 95.45 \% \\
\text { D: } 11 / 15 / 13 \\
\end{array}$ & $\begin{array}{l}\text { History Uni } \\
\text { P. } 23 \\
\text { Ave: } 82.47 \% \\
\text { D: } 11 / 15 / 13 \\
\end{array}$ & \\
\hline \begin{tabular}{|l|}
14020400 \\
\end{tabular} & 300 & 118 & & 50 & 70 & 45 & 43 & 55 & 23 & \\
\hline 14035000 & 300 & 146 & 25 & 50 & 90 & 27 & 45 & 55 & 23 & \\
\hline 14035800 & 0 & 131 & & 50 & 80 & 45 & 43 & 50 & 23 & \\
\hline 14063300 & 0 & 131 & & 50 & 90 & 45 & 43 & 50 & & \\
\hline 14075500 & 300 & 134 & & 50 & 90 & 37 & 45 & 55 & 23 & \\
\hline 14109400 & 300 & 133 & 25 & 50 & 90 & 37 & 45 & 55 & 23 & \\
\hline 14197900 & 0 & 134 & & 50 & 90 & 37 & 45 & 55 & 23 & \\
\hline 14201300 & 200 & 93 & & 50 & 60 & 39 & 45 & 50 & 0 & \\
\hline 14211500 & 300 & 134 & 25 & 50 & 90 & 45 & 45 & 55 & 23 & \\
\hline 14302900 & 100 & 120 & & 0 & 0 & 0 & 0 & 45 & 7 & \\
\hline 14319000 & 0 & 116 & & 50 & 60 & 25 & 33 & 55 & 19 & \\
\hline 14333600 & 300 & 131 & & 50 & 60 & 45 & 19 & 50 & 23 & \\
\hline 14376600 & 300 & 118 & & 50 & $E$ & 29 & 45 & 45 & 23 & \\
\hline 14462300 & 300 & 146 & & 50 & 90 & 27 & 45 & 55 & 23 & \\
\hline 15097400 & 300 & 128 & & 50 & 90 & 45 & 45 & 55 & 23 & \\
\hline 15103900 & 0 & 137 & 25 & 50 & 90 & 37 & 17 & 55 & 23 & \\
\hline 15132100 & 300 & 116 & 25 & 50 & 60 & 45 & 45 & 55 & 23 & \\
\hline 15139500 & 200 & 118 & 25 & 50 & 23 & 45 & 45 & 55 & 20 & \\
\hline 15225300 & 100 & 90 & 25 & 50 & 0 & 0 & 0 & 50 & 0 & \\
\hline 15225800 & 300 & 134 & & 50 & 60 & 43 & 21 & 50 & 23 & \\
\hline 15227000 & 300 & 134 & & 50 & 90 & 45 & 45 & 55 & 23 & \\
\hline 15240500 & 100 & 131 & 25 & 50 & 33 & 45 & 21 & 35 & 9 & \\
\hline 15252700 & 300 & 116 & & 50 & 90 & 25 & 25 & 50 & 23 & \\
\hline 15256600 & 300 & 116 & 25 & 50 & 90 & 45 & 45 & 55 & 23 & \\
\hline 525296400 & 300 & 134 & 25 & 50 & $E$ & 43 & 15 & 55 & 23 & \\
\hline 345200 & 300 & 97 & & 50 & 90 & 45 & 23 & 55 & 23 & \\
\hline 16015400 & 300 & 128 & & 50 & 47 & 43 & 0 & 50 & 23 & \\
\hline 99637600 & 200 & 0 & & 50 & 60 & 45 & 45 & 55 & 23 & \\
\hline 99658645 & 300 & 130 & & 50 & 60 & 45 & 45 & 55 & 23 & \\
\hline 99663197 & 300 & 118 & & 50 & $E$ & 31 & 45 & 55 & 0 & \\
\hline 99675028 & 100 & 0 & & & 0 & 21 & 0 & & 0 & \\
\hline 99717071 & 100 & 130 & & 50 & 50 & 45 & 45 & 55 & 23 & \\
\hline 99746999 & 200 & 137 & & 50 & 90 & 41 & 45 & 55 & 23 & \\
\hline
\end{tabular}


Pleasant Grove High School

Forster, Jessica

The Elements \& Principles of Floral Design - Period 2

\begin{tabular}{|c|c|c|c|c|c|c|c|c|c|c|}
\hline Cludent ID: & $\begin{array}{l}\text { History of } \\
\text { P. } 45 \\
\text { Ave: } 66.02 \% \\
\text { D. } 11 / 15 / 13\end{array}$ & $\begin{array}{l}\text { Holiday Par } \\
\text { P. } 150 \\
\text { Ave: } 83.64 \% \\
\text { D. } 11 / 5 / 13\end{array}$ & $\begin{array}{l}\text { October Cri } \\
\text { P. } 50 \\
\text { Ave: } 85.52 \% \\
\text { D: } 10 / 25 / 13 \\
\end{array}$ & $\begin{array}{l}\text { October Rot } \\
\text { P. } 45 \\
\text { Ave: } 93.47 \% \\
\text { D: } 10 / 24 / 13 \\
\end{array}$ & $\begin{array}{l}\text { Ch } 13 \text { Book } \\
\text { P. } 165 \\
\text { Ave: } 96.66 \% \\
\text { D: } 10 / 16 / 13 \\
\end{array}$ & \begin{tabular}{|l|} 
FFA Pamphle \\
P. 100 \\
Ave: $91.85 \%$ \\
D: $10 / 11 / 13$ \\
\end{tabular} & $\begin{array}{l}\text { 1stQtr Bath } \\
\text { P. } 0 \\
\text { Ave: } 0.00 \% \\
\text { D: } 10 / 11 / 13 \\
\end{array}$ & $\begin{array}{l}\text { FFA Activit } \\
\text { P. } 300 \\
\text { Ave: } 80.81 \% \\
\text { D: } 10 / 11 / 13 \\
\end{array}$ & $\begin{array}{l}\text { Sept Critiq } \\
\text { P. } 50 \\
\text { Ave: } 94.00 \% \\
\text { D: } 10 / 3 / 13 \\
\end{array}$ & \\
\hline 14020400 & 23 & 139 & 0 & 42 & 165 & 91 & & 300 & 39 & \\
\hline 14035000 & 33 & 138 & 50 & 45 & 165 & 95 & 20 & 300 & 50 & \\
\hline 14035800 & 25 & 135 & 46 & 40 & 165 & 90 & 20 & 200 & 50 & \\
\hline 14063300 & & 135 & 43 & 40 & & 90 & 20 & 200 & 14 & \\
\hline 14075500 & 40 & 144 & 48 & 45 & 165 & 100 & & 300 & 50 & \\
\hline 14109400 & 39 & 132 & 43 & 45 & 165 & 92 & & 300 & 50 & \\
\hline 14197900 & 37 & 144 & 48 & 42 & 165 & 90 & & 200 & 50 & \\
\hline 14201300 & 36 & 111 & 37 & 45 & 165 & 90 & 20 & 300 & 41 & \\
\hline 14211500 & 37 & 135 & 48 & 44 & 165 & 95 & 20 & 300 & 49 & \\
\hline 14302900 & 30 & 111 & 32 & 32 & 160 & 95 & 20 & 100 & 50 & \\
\hline 14319000 & 26 & 121 & 48 & 45 & 165 & 77 & 20 & 0 & 48 & \\
\hline 14333600 & 27 & 79 & 48 & 42 & 165 & 100 & 20 & 300 & 50 & \\
\hline 14376600 & 37 & 123 & 43 & 40 & 165 & 90 & 20 & 200 & 50 & \\
\hline 14462300 & 37 & 138 & 48 & 45 & 165 & 95 & 20 & 300 & 50 & \\
\hline 15097400 & 37 & 135 & 50 & 45 & 165 & 100 & 20 & 300 & 50 & \\
\hline 15103900 & 27 & 123 & 43 & 42 & 165 & 95 & & 100 & 50 & \\
\hline 15132100 & 16 & 118 & 48 & 45 & 165 & 92 & 20 & 300 & 48 & \\
\hline 15139500 & 31 & 139 & 43 & 30 & 97 & 95 & 20 & 200 & 41 & \\
\hline 15225300 & 16 & 130 & 40 & 42 & 165 & 55 & 20 & 300 & 40 & \\
\hline 15225800 & 24 & 139 & 48 & 45 & 165 & 95 & 20 & 300 & 50 & \\
\hline 15227000 & 37 & 135 & 48 & 44 & 165 & 90 & 20 & 300 & 49 & \\
\hline 15240500 & 28 & 79 & 48 & 44 & 165 & 92 & 20 & 200 & 48 & \\
\hline 15252700 & 24 & 130 & 48 & 45 & 165 & 95 & & 300 & 46 & \\
\hline 15256600 & 25 & 118 & 48 & 45 & 165 & 100 & 20 & 300 & 50 & \\
\hline 15296400 & 25 & 139 & 48 & 44 & 165 & 95 & 20 & 300 & 50 & \\
\hline$\rightarrow 345200$ & 25 & 85 & 50 & 45 & 165 & 95 & & 300 & 48 & \\
\hline 16015400 & 33 & 132 & 50 & 42 & 78 & 100 & 20 & 300 & 50 & \\
\hline 99637600 & 37 & 135 & 48 & 40 & 165 & 80 & 20 & 100 & 48 & \\
\hline 99658645 & 38 & 108 & 43 & 40 & 165 & 90 & 20 & 300 & 50 & \\
\hline 99663197 & 37 & 149 & 38 & 44 & & 95 & & 300 & 49 & \\
\hline 99675028 & 0 & 121 & 0 & 45 & & 82 & & 100 & 48 & \\
\hline 99717071 & & 108 & 48 & 45 & & 100 & 20 & 200 & 50 & \\
\hline \begin{tabular}{|l|}
99746999 \\
\end{tabular} & 34 & 132 & 40 & 24 & 165 & 95 & 20 & 200 & 45 & \\
\hline
\end{tabular}


Forster, Jessica

Pleasant Grove High School

The Elements \& Principles of Floral Design - Period 2

\begin{tabular}{|c|c|c|c|c|c|c|c|c|c|c|}
\hline fudent ID: & $\begin{array}{l}\text { September } R \\
\text { P. } 90 \\
\text { Ave: } 60.47 \% \\
\text { D: } 10 / 2 / 13 \\
\end{array}$ & $\begin{array}{l}\text { FFA "Why ar } \\
\text { P. } 0 \\
\text { Ave: } 0.00 \% \\
\text { D. } 10 / 2 / 13\end{array}$ & $\begin{array}{l}\text { FFA New Hor } \\
\text { P: } 40 \\
\text { Ave: } 90.53 \% \\
\text { D: } 10 / 1 / 13 \\
\end{array}$ & $\begin{array}{l}\text { Sept Retail } \\
\text { P. } 45 \\
\text { Ave: } 93.75 \% \\
\text { D: } 9 / 30 / 13 \\
\end{array}$ & $\begin{array}{l}\text { Note book Ch } \\
\text { P. } 40 \\
\text { Ave: } 85.83 \% \\
\text { D: } 9 / 27 / 13 \\
\end{array}$ & $\begin{array}{l}\text { Eements \& } \\
\text { P. } 25 \\
\text { Ave: } 78.38 \% \\
\text { D: } 9 / 20 / 13 \\
\end{array}$ & $\begin{array}{l}\text { Business Ag } \\
\text { P. } 20 \\
\text { Ave: } 88.28 \% \\
\text { D: } 9 / 17 / 13 \\
\end{array}$ & $\begin{array}{l}\text { FFA Movie N } \\
\text { P. } 10 \\
\text { Ave: } 93.94 \% \\
\text { D: } 9 / 13 / 13 \\
\end{array}$ & $\begin{array}{l}\text { Unit 1 Exam } \\
\text { P. } 24 \\
\text { Ave: } 74.22 \% \\
\text { D: } 9 / 4 / 13 \\
\end{array}$ & \\
\hline 14020400 & 60 & & 40 & 45 & 38 & 20 & 20 & 10 & 20 & \\
\hline 14035000 & 60 & & 38 & 45 & 38 & 20 & 20 & 10 & 21 & \\
\hline 14035800 & 50 & & 34 & 41 & 32 & 13 & 5 & 10 & 15 & \\
\hline 14063300 & 60 & & 40 & 39 & 30 & 13 & 20 & 10 & 17 & \\
\hline 14075500 & 90 & & 40 & 45 & 40 & 23 & 20 & 10 & & \\
\hline 14109400 & 45 & & 40 & 39 & 31 & 15 & 20 & 10 & 21 & \\
\hline 14197900 & 90 & & 38 & 45 & 34 & $E$ & 20 & 10 & 18 & \\
\hline 14201300 & 0 & & 37 & 27 & 33 & 15 & 20 & 10 & 17 & \\
\hline 14211500 & 60 & & 40 & 45 & 38 & 25 & 20 & 10 & 15 & \\
\hline 14302900 & 18 & & 38 & 43 & 23 & 18 & 0 & 0 & 18 & \\
\hline 14319000 & 27 & & 40 & 41 & 30 & 13 & 20 & 10 & 14 & \\
\hline 14333600 & 38 & & 36 & 43 & 36 & 23 & 20 & 10 & 16 & \\
\hline 14376600 & $E$ & & 27 & 35 & 36 & 15 & 0 & 10 & 15 & \\
\hline 14462300 & 40 & & 38 & 45 & 40 & 20 & 20 & 10 & 21 & \\
\hline 15097400 & 90 & & 40 & 45 & 35 & 23 & 20 & 10 & 19 & \\
\hline 15103900 & 40 & & 39 & 45 & 35 & 18 & 20 & 10 & 19 & \\
\hline 15132100 & 80 & & 34 & 45 & 36 & 18 & 20 & 10 & 16 & \\
\hline 15139500 & 33 & & 39 & 43 & 15 & 20 & 20 & 10 & 19 & \\
\hline 15225300 & 45 & & 35 & 39 & 31 & 13 & 20 & 10 & 16 & \\
\hline 15225800 & 60 & & 38 & 45 & 36 & 22 & 20 & 10 & 18 & \\
\hline 15227000 & 50 & & 40 & 39 & 34 & 25 & 20 & 10 & 21 & \\
\hline 15240500 & 33 & & 36 & 43 & 30 & 23 & 20 & 10 & 19 & \\
\hline 15252700 & 85 & & 38 & 41 & 36 & 23 & 0 & 10 & 17 & \\
\hline 15256600 & 60 & & 38 & 43 & 38 & 18 & 20 & 10 & 19 & \\
\hline 15296400 & 60 & & 36 & 43 & 38 & 22 & 20 & 10 & 19 & \\
\hline 3345200 & 73 & & 39 & 45 & 38 & 23 & 20 & 10 & 21 & \\
\hline 16015400 & 50 & 10 & 40 & 45 & 34 & 23 & 20 & 10 & 18 & \\
\hline 99637600 & 50 & & 34 & 43 & 37 & 23 & 20 & 10 & 19 & \\
\hline 99658645 & 60 & & 38 & 45 & 40 & 22 & 20 & 10 & 17 & \\
\hline 99663197 & $E$ & 10 & 28 & 37 & 37 & 15 & $E$ & 10 & 17 & \\
\hline 99675028 & 0 & & 0 & & 36 & 18 & 20 & 0 & 15 & \\
\hline 99717071 & 90 & & 39 & 43 & 36 & 22 & 20 & 10 & 15 & \\
\hline 99746999 & 90 & & 38 & 43 & 32 & 23 & 20 & 10 & 18 & \\
\hline
\end{tabular}


Forster, Jessica Average: $84.47 \%$

The Elements \& Principles of Floral Design - Period 2

\begin{tabular}{|c|c|c|c|c|c|c|c|c|}
\hline C & $\begin{array}{l}\text { Unit } 1 \text { Stud } \\
\text { P. } 29 \\
\text { Ave: } 97.81 \% \\
\end{array}$ & $\begin{array}{l}\text { Unit } 1 \text { Revi } \\
\text { P. } 30 \\
\text { Ave: } 98.44 \% \\
\text { D. } 8 / 30 / 13\end{array}$ & $\begin{array}{l}\text { Flower Diss } \\
\text { P. } 25 \\
\text { Ave: } 74.91 \% \\
\text { D: } 8 / 26 / 13\end{array}$ & \begin{tabular}{|l} 
Safety Test \\
P 30 \\
Ave: $100.00 \%$ \\
D: $8 / 23 / 13$
\end{tabular} & $\begin{array}{l}\text { Letter to } M \\
\text { P. } 25 \\
\text { Ave: } 96.25 \% \\
\text { D: } 8 / 22 / 13\end{array}$ & $\begin{array}{l}\text { Cut Flower } \\
\text { P. } 25 \\
\text { Ave: } 93.50 \% \\
\text { D: } 8 / 21 / 13 \\
\end{array}$ & $\begin{array}{l}\text { Signed Syll } \\
\text { P. } 25 \\
\text { Ave: } 100.00 \% \\
\text { D: } 8 / 21 / 13 \\
\end{array}$ & \\
\hline student ID: & D: $9 / 4 / 13$ & D: $8 / 30 / 13$ & & 30 & 25 & 23 & 25 & \\
\hline 14020400 & 29 & 30 & \begin{tabular}{|l}
20 \\
19
\end{tabular} & 30 & 25 & 25 & 25 & \\
\hline 14035000 & 29 & 30 & $\frac{19}{19}$ & 30 & 25 & 24 & 25 & \\
\hline 14035800 & 17 & 30 & $\frac{19}{20}$ & 30 & 25 & 25 & 25 & \\
\hline 14063300 & 29 & 30 & $\frac{20}{23}$ & 30 & 25 & 25 & 25 & \\
\hline 14075500 & 30 & 30 & $\frac{23}{18}$ & 30 & 25 & 25 & 25 & \\
\hline 14109400 & 30 & 30 & $\frac{18}{20}$ & 30 & 25 & 23 & 25 & \\
\hline 14197900 & 29 & 30 & $\frac{20}{18}$ & 30 & 25 & 24 & 25 & \\
\hline 14201300 & 29 & 30 & $\frac{18}{24}$ & 30 & 25 & 23 & 25 & \\
\hline 14211500 & 29 & 30 & $\frac{24}{14}$ & 30 & 0 & & 25 & \\
\hline 14302900 & 29 & 30 & $\frac{14}{21}$ & 30 & 25 & 25 & 25 & \\
\hline 14319000 & 29 & 30 & $\frac{21}{17}$ & 30 & 25 & 16 & 25 & \\
\hline 14333600 & 29 & 30 & $\frac{17}{19}$ & 30 & 25 & 23 & 25 & \\
\hline 14376600 & 12 & & $\frac{19}{18}$ & 30 & 25 & 25 & 25 & \\
\hline 14462300 & 29 & 30 & $\frac{18}{18}$ & 30 & 25 & 25 & 25 & \\
\hline 15097400 & 29 & 30 & $\frac{18}{17}$ & 30 & 25 & 25 & 25 & \\
\hline 15103900 & 29 & 21 & $\frac{17}{19}$ & 30 & 25 & 25 & 25 & 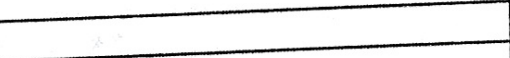 \\
\hline 15132100 & 30 & 30 & $\frac{19}{21}$ & 30 & 25 & 0 & 25 & \\
\hline 15139500 & 29 & 30 & $\frac{21}{20}$ & 30 & 25 & 19 & 25 & \\
\hline 15225300 & 29 & 30 & $\frac{20}{17}$ & 30 & 25 & 25 & 25 & \\
\hline 15225800 & 30 & 30 & $\frac{17}{21}$ & 30 & 25 & 25 & 25 & \\
\hline 15227000 & 29 & 30 & $\frac{21}{17}$ & 30 & 25 & 25 & 25 & \\
\hline 15240500 & 29 & 30 & $\frac{17}{15}$ & 30 & $E$ & 25 & $E$ & \\
\hline 15252700 & 29 & 30 & $\frac{15}{17}$ & 30 & 25 & 25 & 25 & \\
\hline 15256600 & 30 & 30 & $\frac{17}{17}$ & 30 & 25 & 25 & 25 & \\
\hline 5296400 & 30 & 30 & $\frac{17}{24}$ & 30 & 25 & 25 & 25 & \\
\hline 3345200 & 29 & $\frac{30}{30}$ & $\frac{24}{18}$ & 30 & 25 & 24 & 25 & \\
\hline 16015400 & 29 & $\frac{30}{30}$ & $\frac{18}{17}$ & 30 & 25 & 25 & 25 & \\
\hline 99637600 & 29 & \begin{tabular}{|l}
30 \\
30
\end{tabular} & $\frac{17}{18}$ & 30 & 25 & 25 & 25 & \\
\hline 99658645 & 30 & \begin{tabular}{|l|}
30 \\
30
\end{tabular} & $\frac{19}{19}$ & 30 & 25 & 25 & 25 & \\
\hline 99663197 & 29 & $\frac{30}{24}$ & 17 & 30 & 20 & 25 & 25 & \\
\hline 99675028 & 29 & $\frac{24}{30}$ & 18 & 30 & 25 & 25 & 25 & \\
\hline 99717071 & 30 & $\frac{30}{30}$ & 18 & 30 & 25 & 24 & 25 & \\
\hline
\end{tabular}


Support Material 5:

จ Completed SAE Supervision Forms 
Pleasant Grove High School Agriculture Department

Support Material 5: Completed SAE Supervision Forms

Project supervision forms are provided as a record of a meeting between the SAE advisor and the students when a project visit occurs. This form is signed at the time of the project visit and a copy is provided to the student while the original form is kept in the student's permanent data file. This form has proved essential in the past when students have chosen not to follow the instruction of their supervisins SAE advisor.

Included are SAE project visit forms for ten current agriculture students with active SAE projects. 


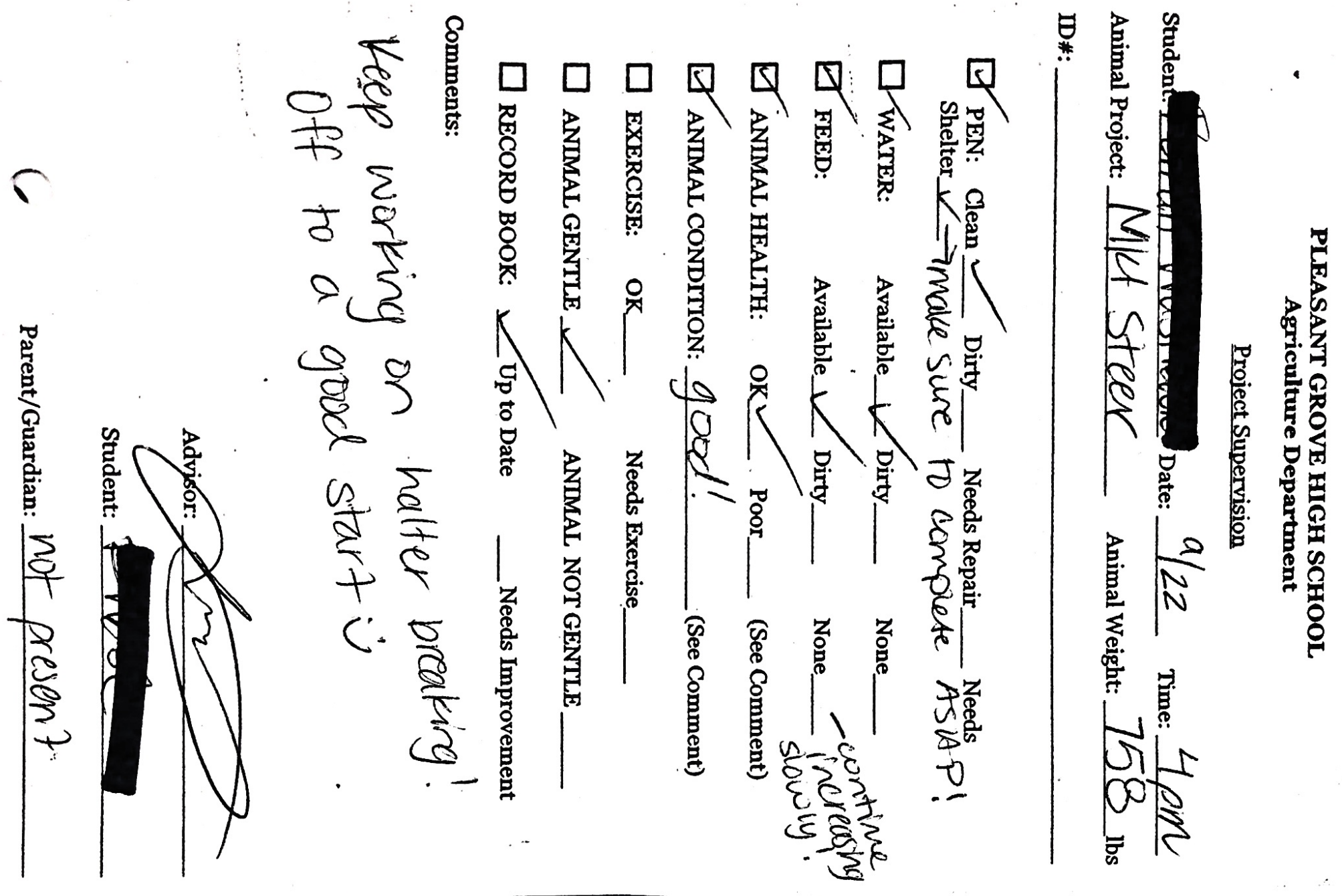

C

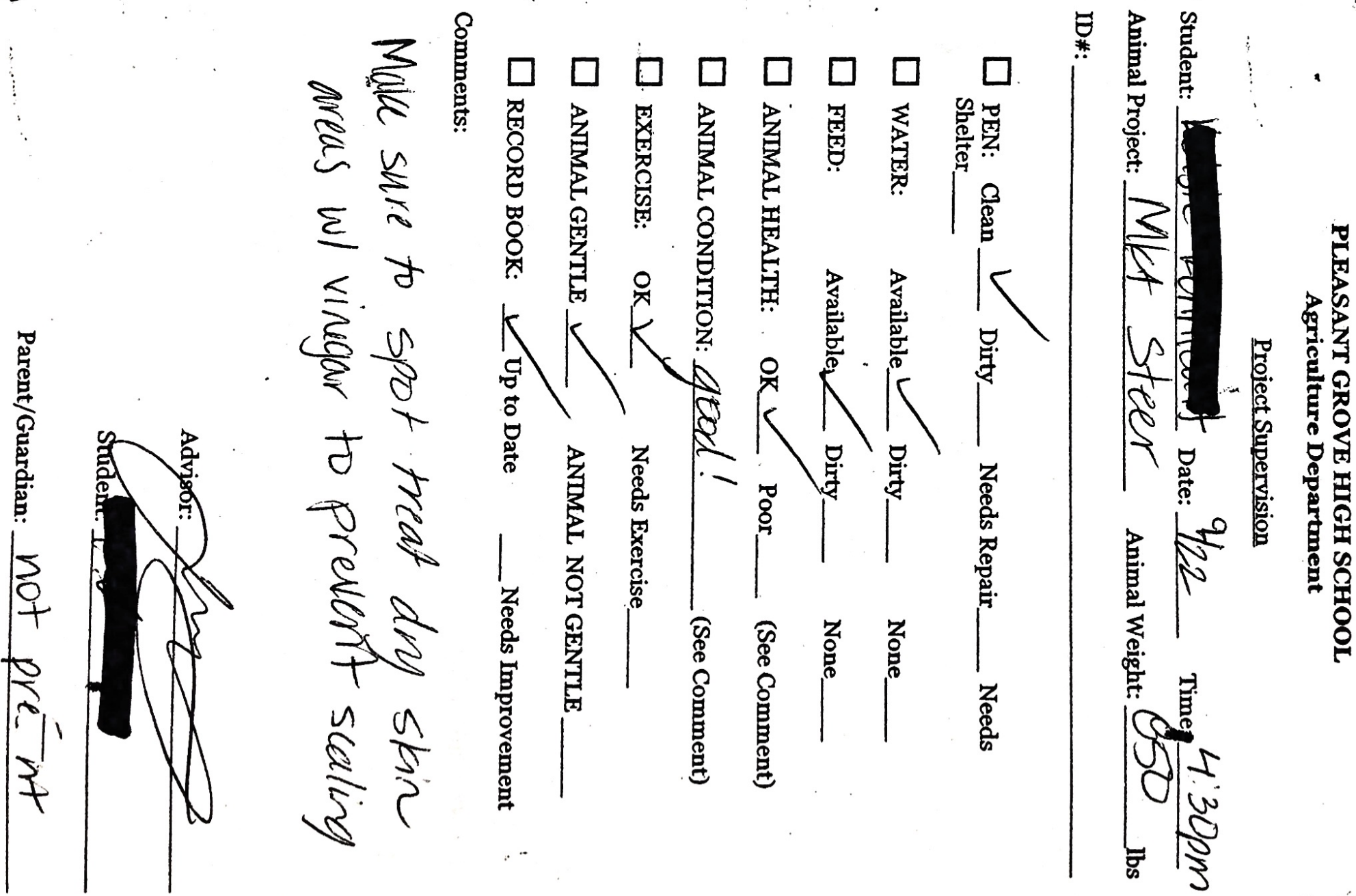



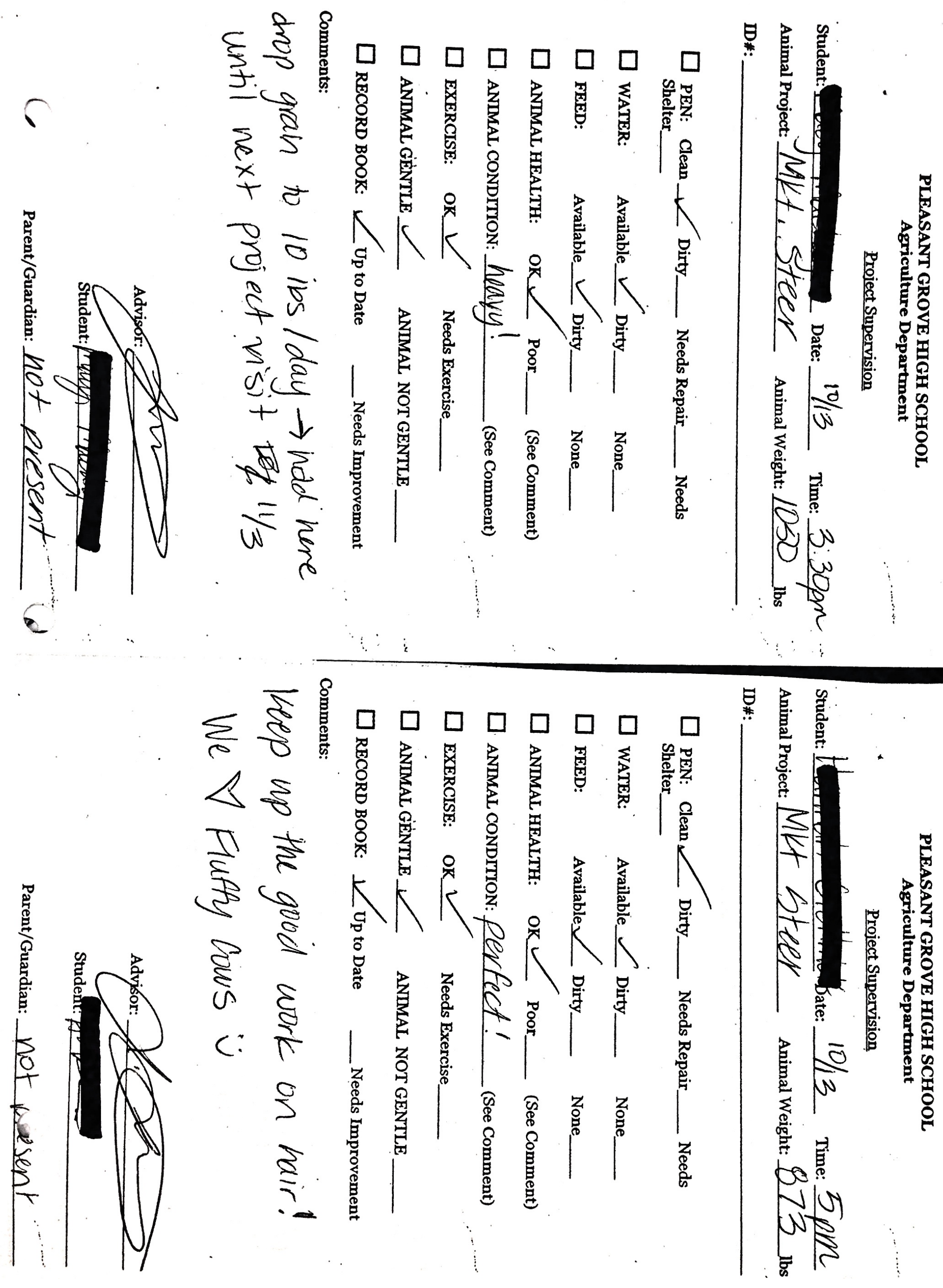


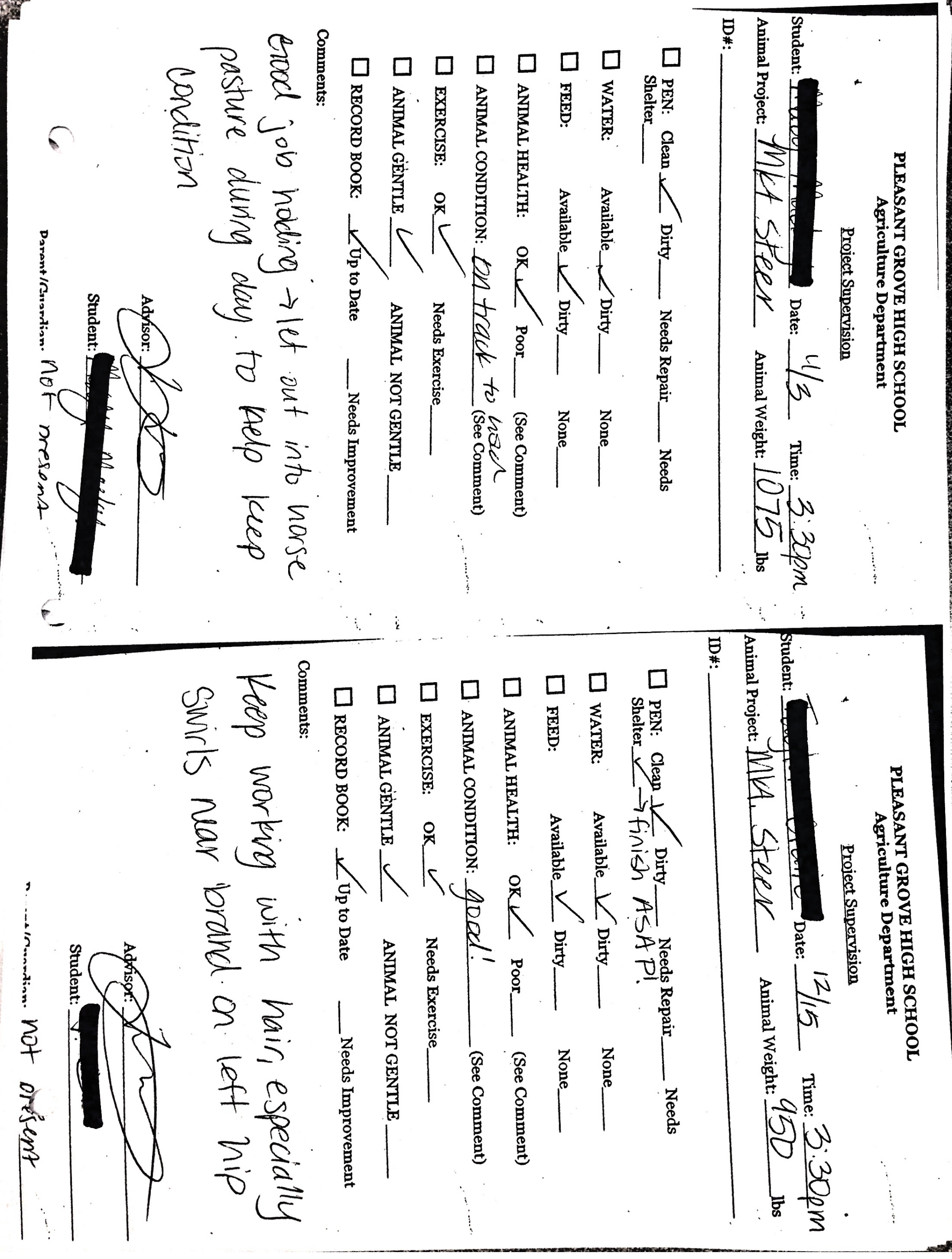




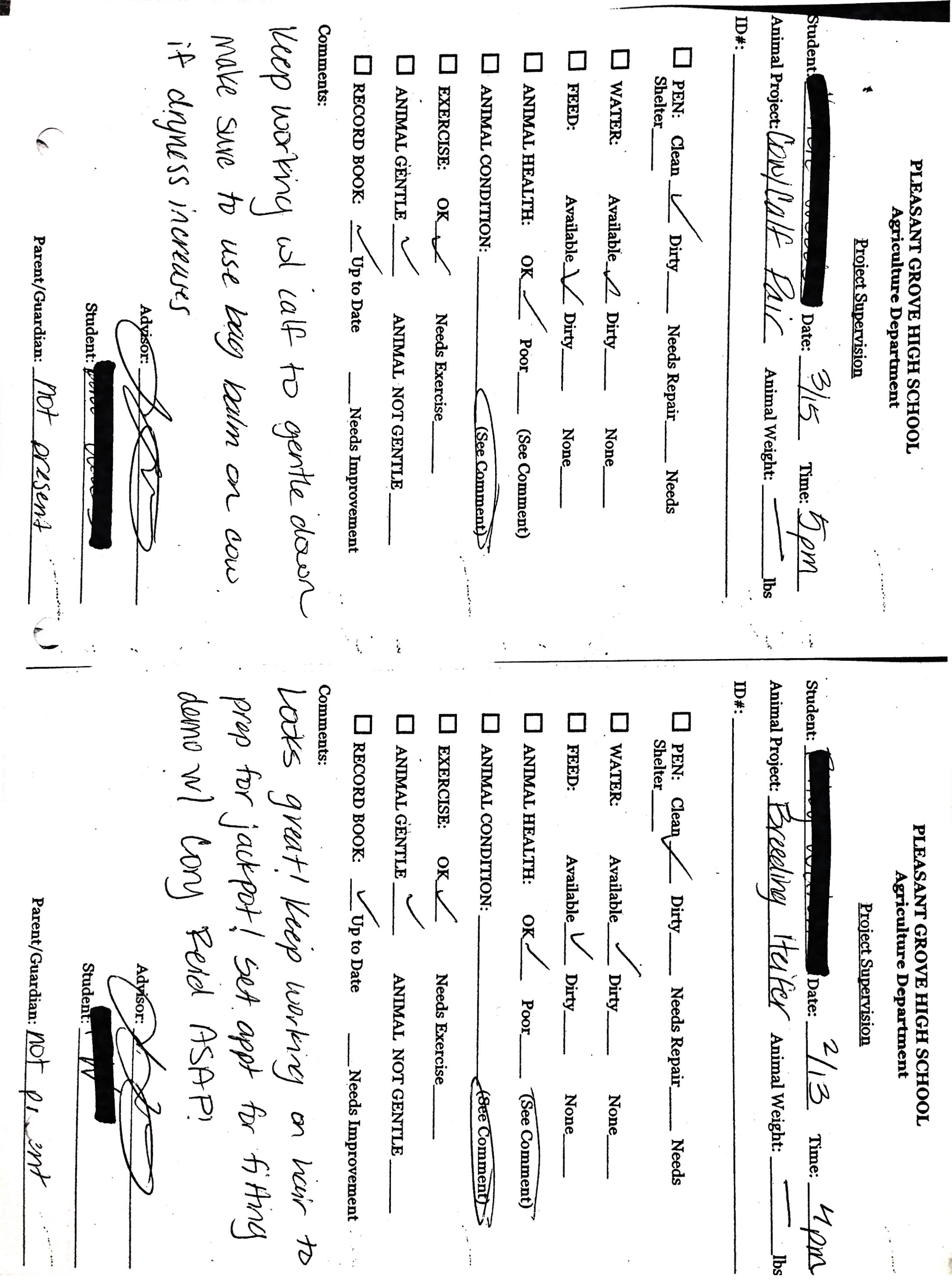



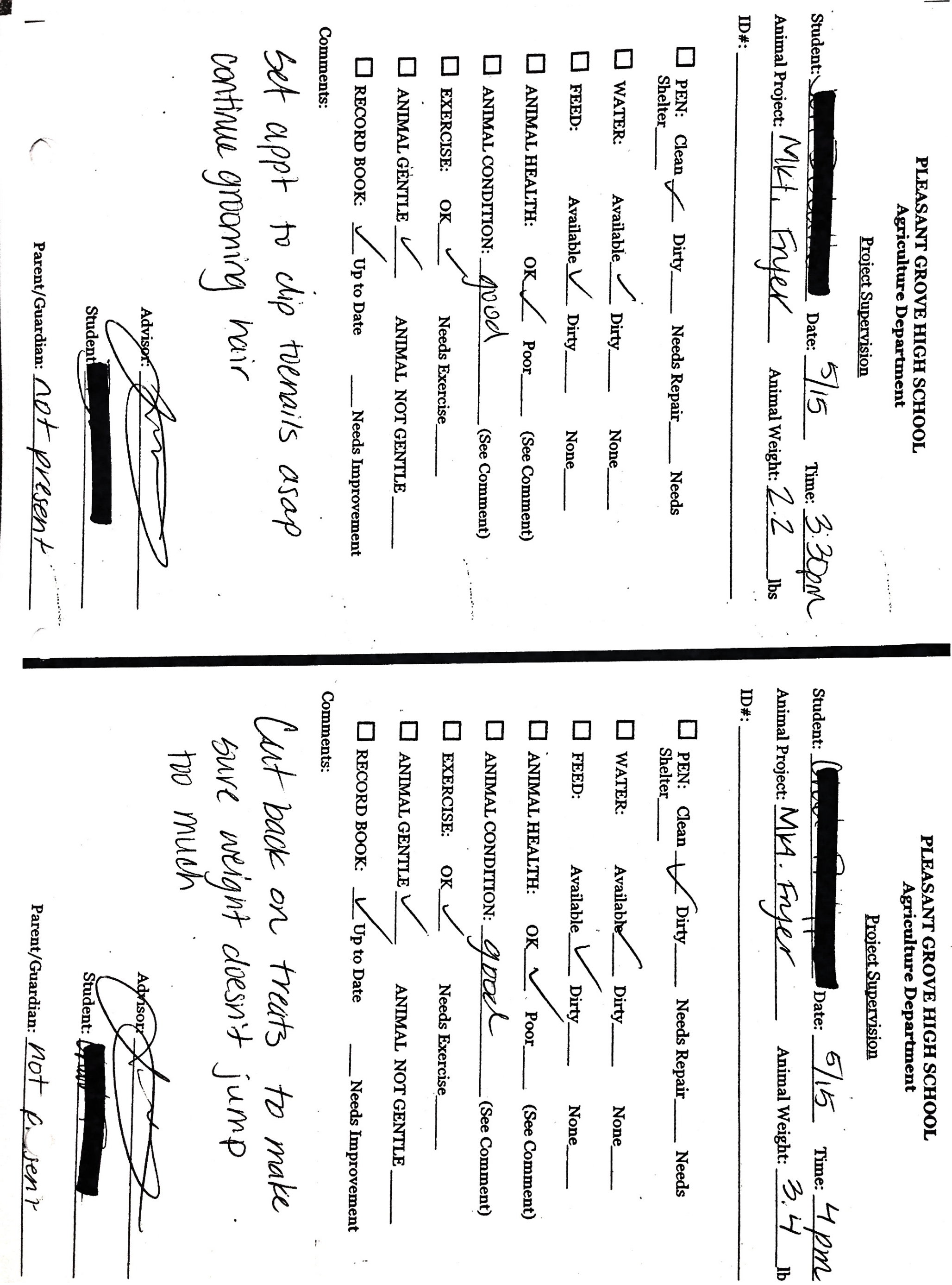
Support Material 6:

SAE Project Statement in Syllabus 


\section{Pleasant Grove High School Agriculture Department}

\section{Support Material 6: SAE Project Statement in Syllabus}

The requirements of the Supervised Agriculture Experience project are clearly listed in the course syllabus that is signed by the student and a parent/guardian at the beginning of each school year. Each student is required to complete at least three hours each month on their SAE proejct, which is then documented in the Record Book. 


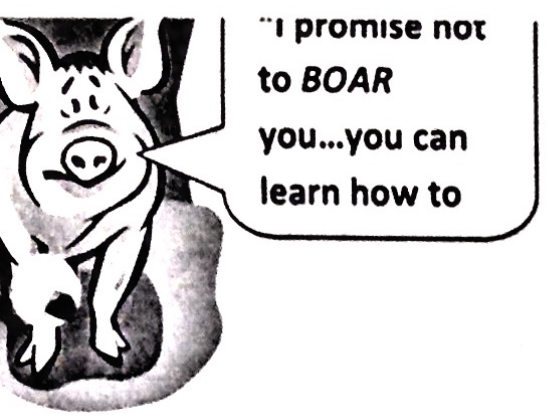

\section{Pleasant Grove High School Agriculture Department \\ Course Expectations 2013-2014}

Instructor: Ms. Forster

Office Phone: 916-686-0230, ext., 4933

Email: jforster@egusd.net

XVIII. Course Description: Applied General Science

This course will cover units such as Astronomy, Earth's Interior, Oceans, Plate Tectonics, etc. Future Farmers of America (FFA) will also be an integral part of this course. Students will be introduced to the FFA Program, California Agriculture, Careers in Agriculture, and much more.

XIX. Class Policies:

1. Be in your seat and ready to work, within one minute of the bell ringing. If you're not, you are tardy.

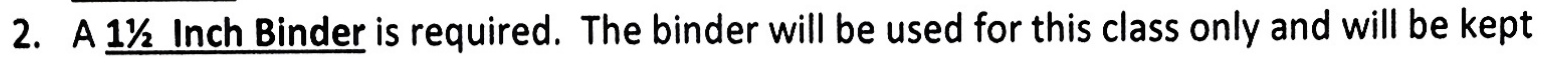
organized and neat. There is a place for you to store this binder in the classroom.

3. Each student must bring a pencil or pen to class every day.

4. No food or drink is allowed in the classroom during class except water.

5. The classroom and Agriculture Department must be kept clean. All cleanup activities will be done prior to students being dismissed.

XX. Grading:

Your grade will be based on the following:

50\% Classroom work. This includes homework, in class assignments etc...

30\% Quizzes/Exams. This includes both semester finals.

10\% FFA Participation

$10 \%$ Supervised Agricultural Experience Project (SAE)

Letter grades will be assigned using the following guidelines:

$\begin{array}{llrl}94 \% \text { and above } & \text { A } & 67-69 \% & \text { D+ } \\ 90-93 \% & \text { A- } & 64-66 \% & \text { D } \\ 87-89 \% & \text { B+ } & 60-63 \% & \text { D- } \\ 84-86 \% & \text { B } & 59 \% \text { and below } & \text { F } \\ 80-83 \% & \text { B- } & & \\ 77-79 \% & \text { C+ } & & \\ 74-76 \% & \text { C } & & \\ 70-73 \% & \text { C- } & & \end{array}$

\section{Note: GRADES WILL BE UPDATED ON TUESDAYS!!!}

XXI. Homework and Class Assignments, $50 \%$ of grade

1. Late assignments will not be accepted (except in emergency situations).

2. In the event that homework is not turned in, students will complete a log in the "No Homework Binder" to document this occurrence.

3. Assignments should be neat and complete.

4. For each day you are absent, you have that many days to make it up.

5. Most weekly homework will be assigned Monday of each week.

XXII. Tests, $\mathbf{3 0} \%$ of grade

1. Tests will be given at the completion of each unit of study.

2. Quizzes may be given periodically as needed. 
3. There will be a Final Exam at the end of each semester.

4. Make up tests are the responsibility of the student! If you have an excused absence on the day of a test, it is your responsibility to make an appointment to take it within 1 week of returning to school - if a test is not made up within one week of the test being administered, a score of zero will be given for that test grade.

5. A student may re-take a previously completed test to attempt to earn a higher grade at any time before the current quarter ends by scheduling an appointment with the instructor for before or after school or during their lunch hour.

XXIII. SAE Projects $-10 \%$ of grade

1. All students are required to have a project that relates to a career in agriculture and requires 3 hours of labor per month.

2. Projects require prior approval of the instructor.

3. This project is $10 \%$ of your grade. If you have more than one $\mathrm{Ag}$ class, you only have to do one SAE project.

XXIV. FFA - $10 \%$ of grade

1. Your enrollment in this class automatically makes you an FFA member however, your level of participation depends on what you get interested in and your desire to get involved with all the various activities offered. If you are a 4- $\mathrm{H}$ member you can definitely be active in both organizations and get a great experience from them!

2. The FFA is an integral part of this class therefore you are required to participate in various activities throughout the semester. FFA is the club within the Agriculture Department.

3. You are required to get 3 FFA credits per quarter and you will usually have more than $\mathbf{1 5}$ opportunities to do this. This should be the easiest part of your grade!

4. Examples of things you can do to get FFA Credits: Attend the once a month Chapter meeting put on by the Officer Team, participate in leadership conferences, public speaking contests, assist with fundraisers such as our Car Wash and much more! There are plenty of opportunities to get these credits!

\section{Student Expectations}

- Responsibility: You are expected to keep track and turn in your assignments on due dates. Excuses and whining are not permitted.

- Respect: All students have the right to learn and achieve without the interference of others.

Students will respect their property and that of others.

Exhibiting prejudice or prejudicial behaviors will NOT be tolerated.

Students are expected to use acceptable and appropriate language. Profanity $\neq$ Respect

$\checkmark$ Interrupting the teacher or another student who is speaking is rude and limits the opportunity for others to learn.

- Classroom Rules: No food or gum in the classroom. Water is allowed in re-closable containers. No hats or sunglasses are to be worn in the classroom. No electronic devices are to be used in the classroom. PGHS dress code will be followed daily.

- Be Prepared: Bring class binder, papers, pens/pencils, and your brain! () 
7. Reminded of the rules and given a choice to abide by rules and told of consequences if
reoccurs.

8. Continued disruptions, will result in an appropriate consequence (parent contact, detention, removal from class, referrals, etc.)

9. If a substitute teacher believes a student has refused to abide by the class rules, the student is required to complete a Substitute Behavior Essay, which is a 100 point assignment.

VI. Miscellaneous

- Academic Code of Conduct will be enforced. Refer to Parent/Student Handbook.

- The instructor has the right to alter, add to, or delete any portion of this class policy with prior notification to the class.

- Papers turned in with NO NAME will not be graded! If an assignment is turned in without a name, students may use a Renaissance Pass or STAR Card to resubmit the assignment.

- Any correspondence (e.g. phone calls, emails, etc.) will be responded within 2 business days of receiving them. If I am out of town on a FFA field trip, response time may be longer.

\section{Applied General Science Ms. Forster}

I have read and understand all the policies and procedures contained herein. Please sign and return to your instructor.

Student Signature

Parent/Guardian Signature
Date

Date 
$a$

Support Material 7:

FFA Statement in

Syllabus 


\section{Pleasant Grove High School Agriculture Department}

\section{Support Material 7: FFA Statement in Syllabus}

The requirements for FFA participation are clearly listed in the course syllabus that is signed by the student and a parent/guardian at the beginning of each school year. Each student is required to attend at least two local FFA activities per academic quarter, which is then documented in the Record Book. 


\section{Pleasant Grove High School Agriculture Department \\ Course Expectations 2013-2014}

Instructor: Ms. Forster

Office Phone: 916-686-0230, ext., 4933

Email: jforster@egusd.net

XXVI. Course Description: Applied General Science

This course will cover units such as Astronomy, Earth's Interior, Oceans, Plate Tectonics, etc. Future Farmers of America (FFA) will also be an integral part of this course. Students will be introduced to the FFA Program, California Agriculture, Careers in Agriculture, and much more.

XXVII. Class Policies:

1. Be in your seat and ready to work, within one minute of the bell ringing. If you're not, you are tardy.

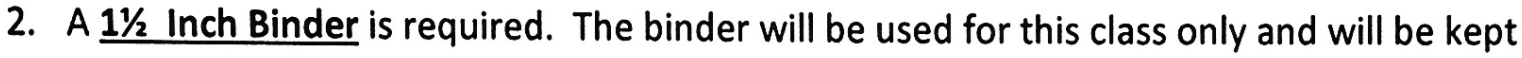
organized and neat. There is a place for you to store this binder in the classroom.

3. Each student must bring a pencil or pen to class every day.

4. No food or drink is allowed in the classroom during class except water.

5. The classroom and Agriculture Department must be kept clean. All cleanup activities will be done prior to students being dismissed.

XXVIII. Grading:

Your grade will be based on the following:

$\mathbf{5 0 \%}$ Classroom work. This includes homework, in class assignments etc...

30\% Quizzes/Exams. This includes both semester finals.

$10 \%$ FFA Participation

10\% Supervised Agricultural Experience Project (SAE)

Letter grades will be assigned using the following guidelines:

$\begin{array}{llll}94 \% \text { and above } & \text { A } & 67-69 \% & \text { D+ } \\ 90-93 \% & \text { A- } & 64-66 \% & \text { D } \\ 87-89 \% & \text { B+ } & 60-63 \% & \text { D- } \\ 84-86 \% & \text { B } & 59 \% \text { and below } & \text { F } \\ 80-83 \% & \text { B- } & & \\ 77-79 \% & \text { C+ } & & \\ 74-76 \% & \text { C } & & \\ 70-73 \% & \text { C- } & & \end{array}$

\section{Note: GRADES WILL BE UPDATED ON TUESDAYS!!!}

XXIX. Homework and Class Assignments, $50 \%$ of grade

1. Late assignments will not be accepted (except in emergency situations).

2. In the event that homework is not turned in, students will complete a log in the "No Homework Binder" to document this occurrence.

3. Assignments should be neat and complete.

4. For each day you are absent, you have that many days to make it up.

5. Most weekly homework will be assigned Monday of each week.

XXX. Tests, $30 \%$ of grade

1. Tests will be given at the completion of each unit of study.

2. Quizzes may be given periodically as needed. 
3. There will be a Final Exam at the end of each semester.

4. Make up tests are the responsibility of the student! If you have an excused absence on the day of a test, it is your responsibility to make an appointment to take it within 1 week of returning to school - if a test is not made up within one week of the test being administered, a score of zero will be given for that test grade.

5. A student may re-take a previously completed test to attempt to earn a higher grade at any time before the current quarter ends by scheduling an appointment with the instructor for before or after school or during their lunch hour.

XXXI. SAE Projects $-10 \%$ of grade

1. All students are required to have a project that relates to a career in agriculture and requires 3 hours of labor per month.

2. Projects require prior approval of the instructor.

3. This project is $10 \%$ of your grade. If you have more than one Ag class, you only have to do one SAE project.

XXXII. FFA $-10 \%$ of grade

1. Your enrollment in this class automatically makes you an FFA member however, your level of participation depends on what you get interested in and your desire to get involved with all the various activities offered. If you are a $4-\mathrm{H}$ member you can definitely be active in both organizations and get a great experience from them!

2. The FFA is an integral part of this class therefore you are required to participate in various activities throughout the semester. FFA is the club within the Agriculture Department.

3. You are required to get $\mathbf{3}$ FFA credits per quarter and you will usually have more than $\mathbf{1 5}$ opportunities to do this. This should be the easiest part of your grade!

4. Examples of things you can do to get FFA Credits: Attend the once a month Chapter meeting put on by the Officer Team, participate in leadership conferences, public speaking contests, assist with fundraisers such as our Car Wash and much more! There are plenty of opportunities to get these credits!

\section{Student Expectations}

- Responsibility: You are expected to keep track and turn in your assignments on due dates. Excuses and whining are not permitted.

- Respect: All students have the right to learn and achieve without the interference of others.

Students will respect their property and that of others.

$\rightarrow$ Exhibiting prejudice or prejudicial behaviors will NOT be tolerated.

Students are expected to use acceptable and appropriate language. Profanity $\neq$ Respect

Interrupting the teacher or another student who is speaking is rude and limits the opportunity for others to learn.

- Classroom Rules: No food or gum in the classroom. Water is allowed in re-closable containers. No hats or sunglasses are to be worn in the classroom. No electronic devices are to be used in the classroom. PGHS dress code will be followed daily.

- Be Prepared: Bring class binder, papers, pens/pencils, and your brain! (-)

\section{Consequences}

Students that REFUSE to follow the above behavioral expectations will be: 
10. Reminded of the rules and given a choice to abide by rules and told of consequences if reoccurs.

11. Continued disruptions, will result in an appropriate consequence (parent contact, detention, removal from class, referrals, etc.)

12. If a substitute teacher believes a student has refused to abide by the class rules, the student is required to complete a Substitute Behavior Essay, which is a 100 point assignment.

VI. Miscellaneous

- Academic Code of Conduct will be enforced. Refer to Parent/Student Handbook.

- The instructor has the right to alter, add to, or delete any portion of this class policy with prior notification to the class.

- Papers turned in with NO NAME will not be graded! If an assignment is turned in without a name, students may use a Renaissance Pass or STAR Card to resubmit the assignment.

- Any correspondence (e.g. phone calls, emails, etc.) will be responded within 2 business days of receiving them. If I am out of town on a FFA field trip, response time may be longer.

\section{Applied General Science Ms. Forster}

I have read and understand all the policies and procedures contained herein. Please sign and return to your instructor.

Student Signature

Parent/Guardian Signature
Date

\section{Date}


Support Material 8:

FFA Program of Activities 


\section{Pleasant Grove High School Agriculture Department}

\section{- Support Material 8: FFA Program of Activities}

At this time, our chapter currently does not have a Chapter Program of Activities. As a part of my Master's project, I have developed a basic Program of Activities for the current school year - it is basic, but gives our parents and students an idea of what to expect in the PGHS Agriculture Department.

It is my hope that next year, our Chapter Officer Team will take ownership of the Program of Actvities, and revamp and reformat it so that it may be competivite at the Regional Program of Activities Career Development Event. 
Support Material 9: Recruitment Program 


\section{Pleasant Grove High School Agriculture Department}

\section{Support Material 9: Recruitment Program}

Recruitment is arguable one of the most important aspects to a successful agriculture program. The first group of people we try to recruite is students. We have a standing agreement with our feeder school (conviniently built next to PGHS) to conduct a recuirment day in all $8^{\text {th }}$ grade sceince classes each March. Our student run Recruitment Committee is responsible for planning the activitis that take place at this recruitment day, which normally include a presentation on opportunities available to students in the agriculture department, shared personal stories of current FFA members, and fun activities that allow $8^{\text {th }}$ graders to connect with current students. Each $8^{\text {th }}$ grade classroom typically has four to five current agriculture students conduct the recruitment activities, which conclude with flyers with information for parents.

Once students have completed the recruitment day activities, our Chapter Officer Team attends the annual $8^{\text {th }}$ Grade Parent Information Night, where different departments present information to the $8^{\text {th }}$ grade parents. Chapter Officers give a five minute run-down of the opportunities avaialbe to students through FFA, and are availabe at the conclusion of the evening to answer any questions from parents.

To retain our current students, we conduct planning sessions in all agriculture classes to ensure students know their options for each grade level, where next year's class schedule is planned, to ensure they are meeting all graduation, higher education, and program requirements. These year plans are required to be signed by a parent/guardian to make them aware of options through the agriculture program.

A copy of our Comprehensive Program Plan and Recruitment Day pamphlet is attached. 


\section{Agriculture Career Pathways \& Academic Plan \\ Pleasant Grove High School}

\begin{tabular}{|c|c|}
\hline $9^{\text {th }}$ Grade & $10^{\text {th }}$ Grade \\
\hline English 9 & English 10 \\
\hline Math & Math \\
\hline Health/Geography & World History \\
\hline Applied Genera & Ag Biology \\
\hline Science & \\
\hline Foreign Language & Foreign Language \\
\hline Physical Education & Physical Education \\
\hline
\end{tabular}

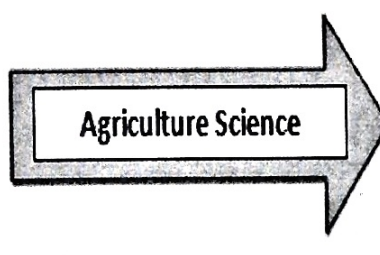

\begin{tabular}{|c|c|}
\hline & \\
\hline $11^{\text {th }}$ Grade & $12^{\text {th }}$ Grade \\
\hline English 11 & English 12 \\
\hline Math & Math \\
\hline U.S. History & Government/Comp Tech \\
\hline Himal Anatomy 8. Physiology & Veterinary science \\
\hline Elective & Economics in Ag \\
\hline Chemistry & Chemistry \\
-or- & -or- \\
Elective & Elective \\
\hline
\end{tabular}

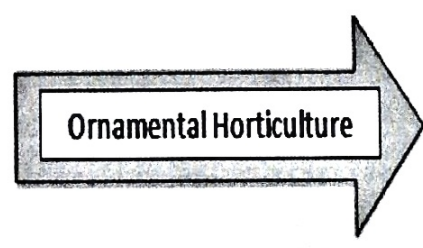

\begin{tabular}{|c|c|}
\hline $11^{\text {th }}$ Grade & $12^{\text {th }}$ Grade \\
\hline English 11 & English 12 \\
\hline Math & Math \\
\hline U.S. History & Government/Comp Tech \\
\hline Foral Design & Ornamental Horticulture \\
\hline Elective & Economics in Ag \\
\hline Chemistry & Chemistry \\
-or- & -or- \\
Elective & Elective \\
\hline
\end{tabular}




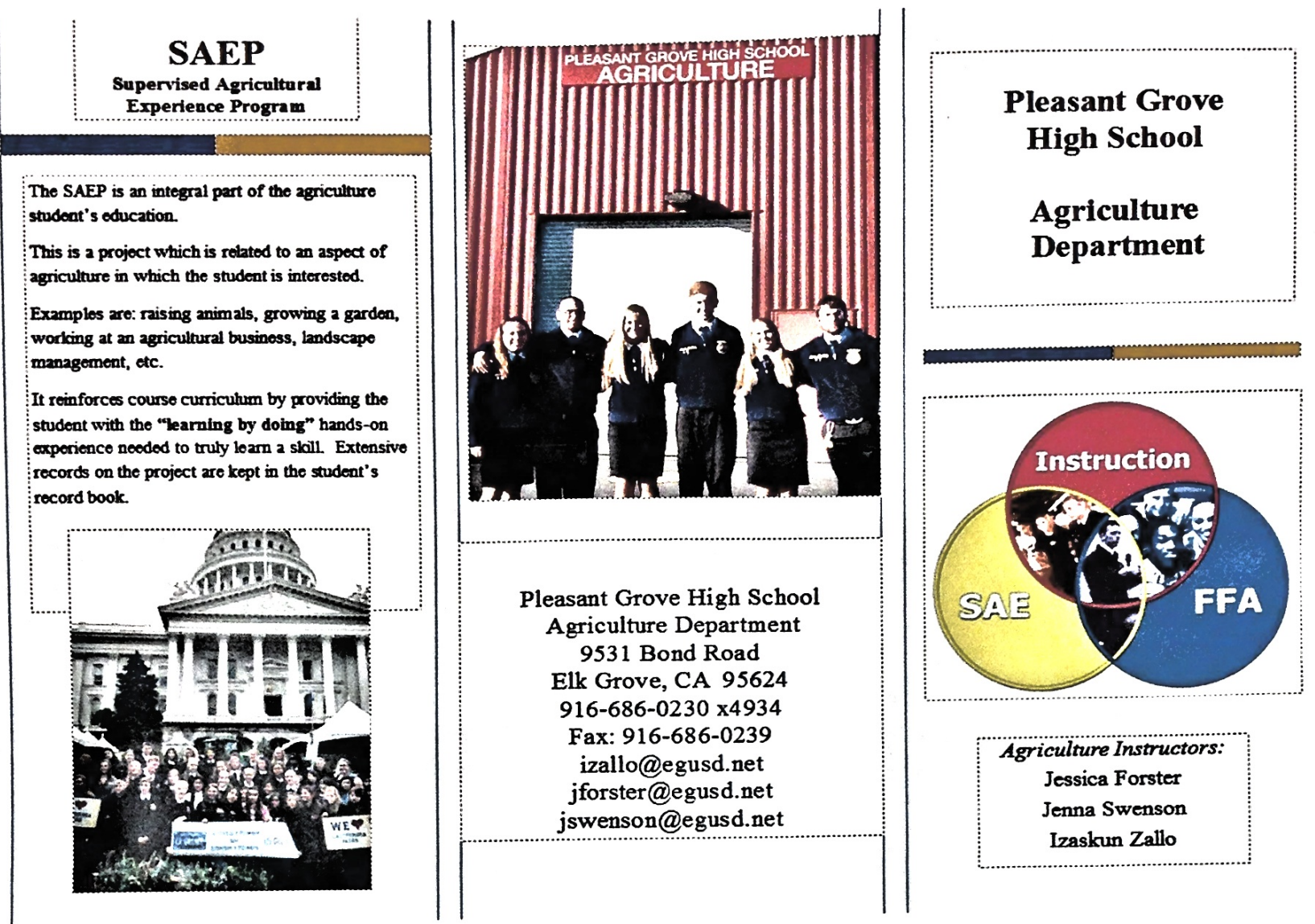

\section{Agriculture Classes

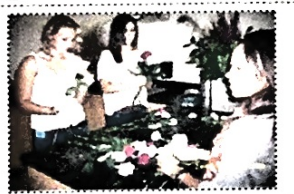

Applied General Science $(9)$

An introduction to earth science, chemistry, forces, energy, \& waves as it pertains to agriculture. Meets UC/ CSU elective requirement and General Science graduation requirement.

Agricultural Biology $(9,10)$

ab science course in molecular \& cellular aspects of Lab scientics of life; chemical \& structural basis of ife; energes of ife; growth \& reproducion in plans \& amimals; evolution of modem plants and domestic livestock Meets UC/CSU lab requirement and Life Science gradurion requirement.

Elements \& Principles of Floral Design (11,12) This course covers theories and principles related to floral design and artistic design. Students will acquire practical sialls and lonowledge in the history, theory, and application of artistic styles and practices used in flor design. Meets UC/CSU Visual \& Performing Art requirement and the Visual and Pefforming Arts graduation requirement.

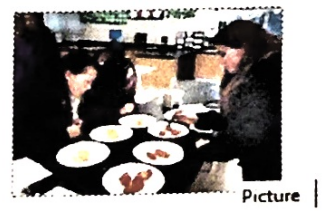

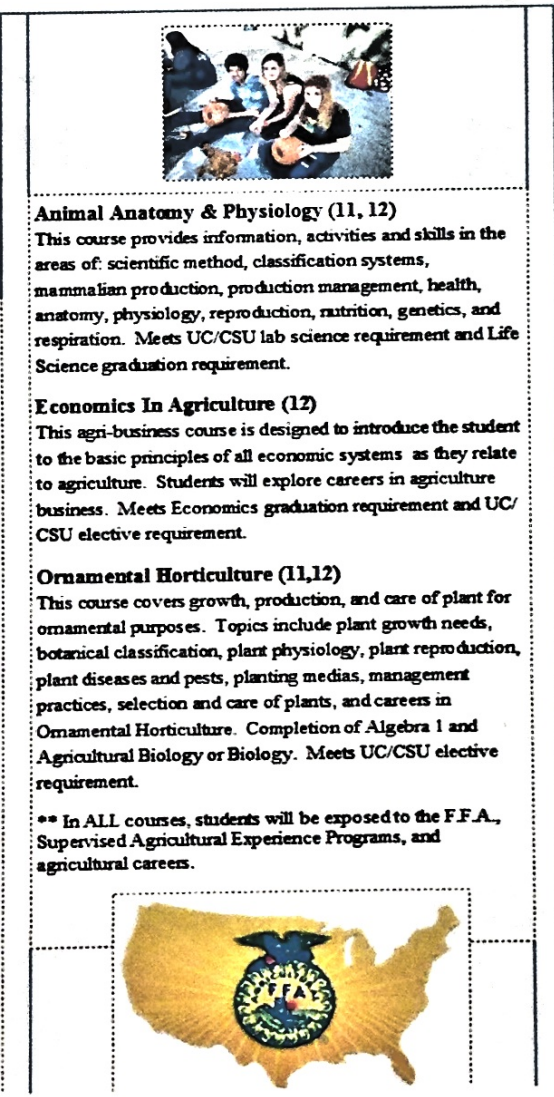

FFA

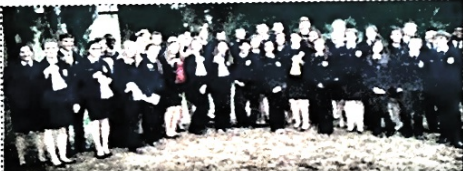

FFA is a national leadership organization of students enrolled in public school vocational agriculture and agribusiness programs in the 50 states. Puerto Rico \& Virgin Islands.

It is an educational, nonprofit organization designed to develop student's agricultural leadership. strengthen student's self-image, improve public speaking skills, obtain college/carcer readiness stills, scholarship, cooperztion, citizenship, and patriotism.

In California, there are approximately 303 high schools participating in FFA. Overall, FFA membership in California totals approximately 70,555 students!

There are over $\mathbf{4 0 0}$ students at Pleasant Grove Figh School.

Premier Leadersht
Personal Growth
Career Success




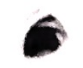

Support Material 10:

FFA Chapter

Scrapbook 


\section{Pleasant Grove High School Agriculture Department}

\section{Support Material 10: FFA Chapter Scrapbook}

Our Chapter Scrapbook is maintained by our Chapter Reporter and an active Scrapbook Committee. The Scrapbook Committee is responsible for taking pictures at events and constructing the scrapbook at monthly committee meetings.

Attacehd are a small sample of some of this year's scrapbook pages.
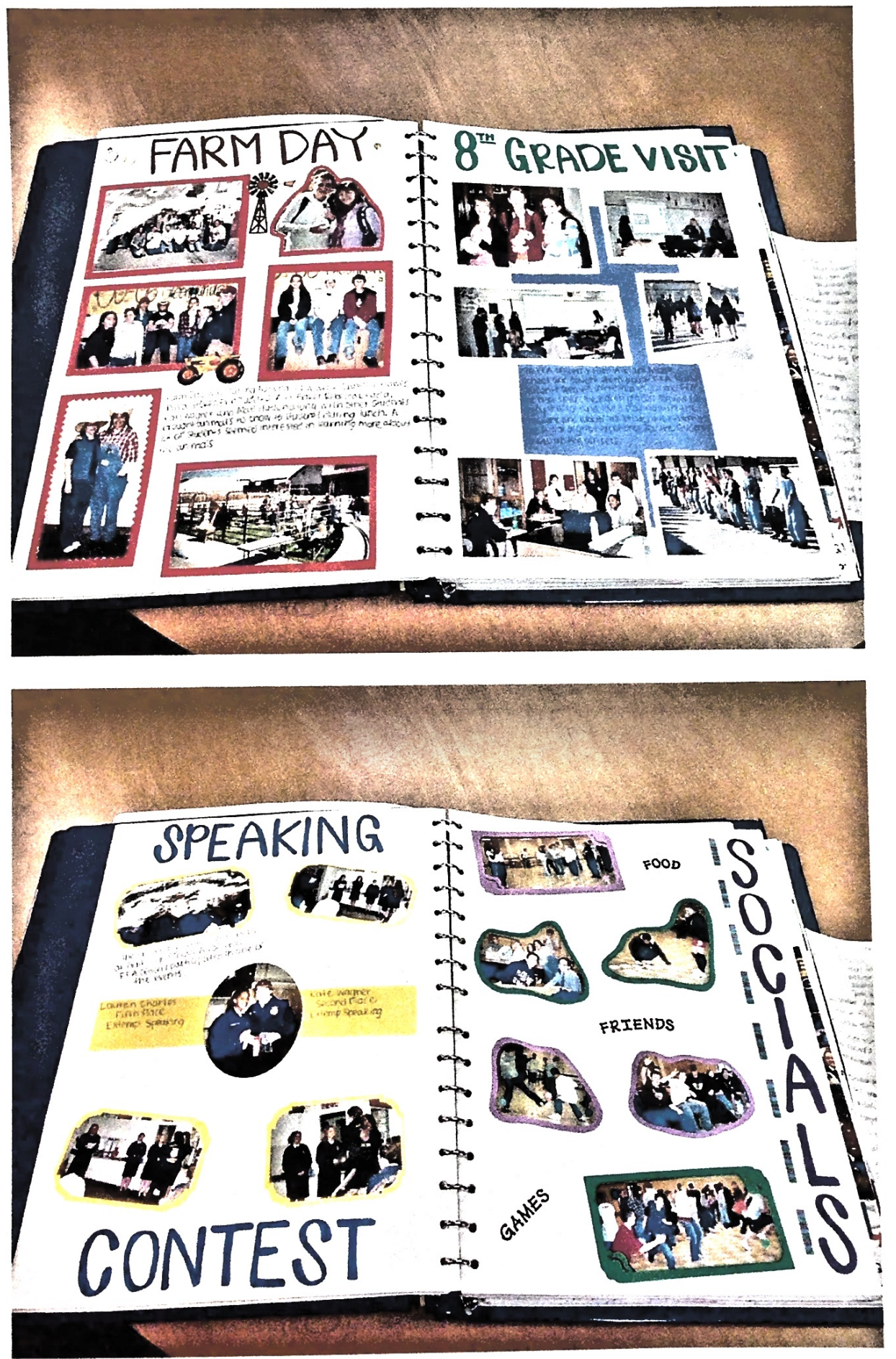

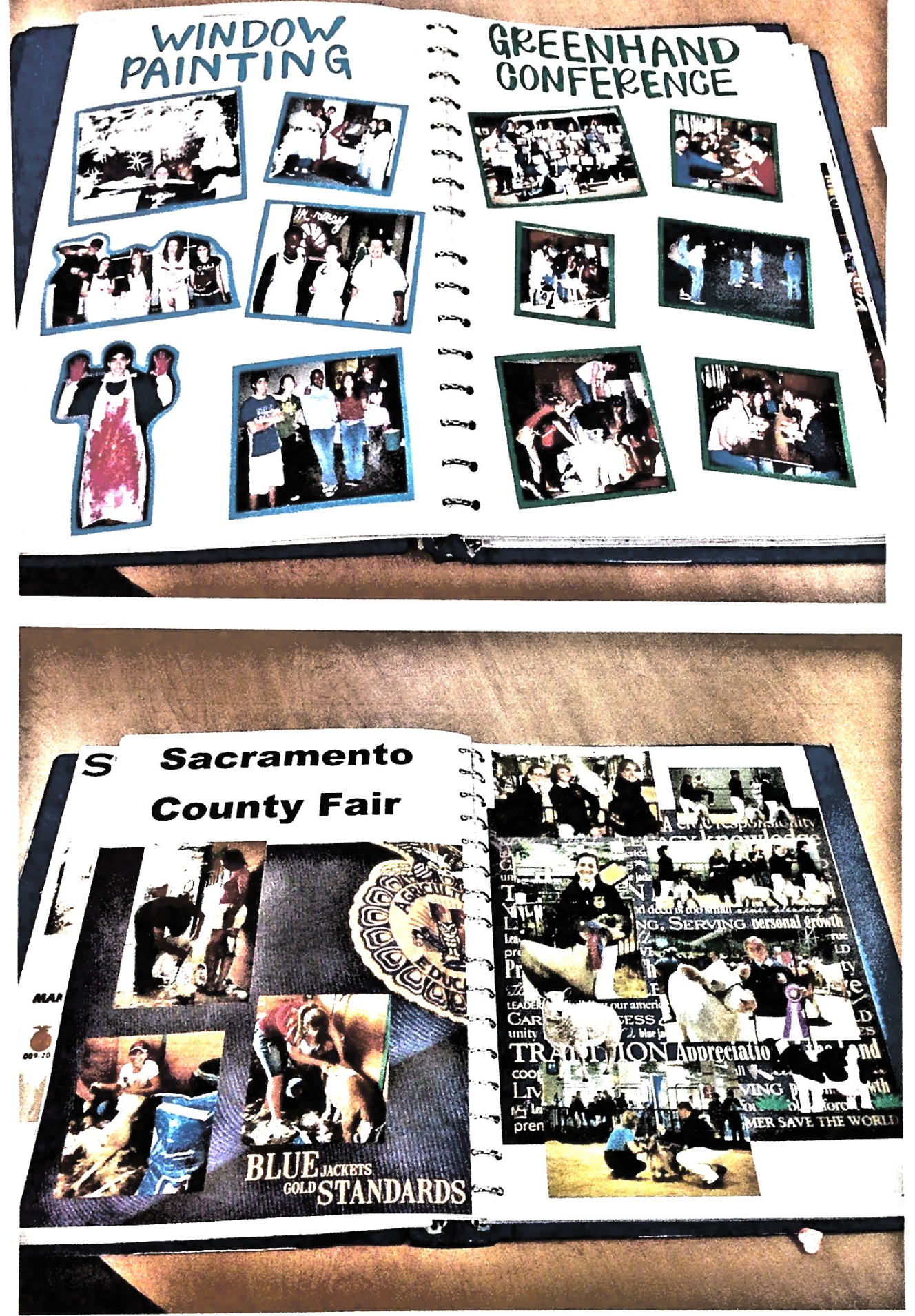


\section{Support Material 11:}

Summer Activities Calendar 


\section{Pleasant Grove High School Agriculture Department}

\section{Support Material 11: Summer Activities Calendar}

Unfortunately, a summer stipend is not available to agriculture instructor within the Elk Grove Unified School District, therefore there is not structured Summer Activities Calendar. Each agriculture teacher does attend the California Agriculture Teacher's Association Summer Conference each year, which consists the only required summer activity.

Since I teach the only Summer ROP Sales/Service and Livestock Class during the summer months, this is how I am able to earn a modified summer stipend. The ROP Office requires there to be 35 non-contract days included in this class, with a minimum of 6 class meetings, time for professional devleopment (earned through CATA Conference), as well as curriculum development. In addition to the class meetings, I conduct project visits throughout the summer, and use this time to update my curriculum for the coming year.

Attached is the information sheet that went out to all student interested in enrolling in Summer ROP, as well as a class calendar for the summer. 


\section{To: Summer Ag R.O.P. Participants}

From: Ms. Forster, Summer Ag R.O.P. Instructor

Thank you for your interest in Summer Ag R.O.P. The purpose of this class is for you to be able to earn class credits (a total of 10 for the summer) in exchange for keeping a record of the work you complete on an existing Supervised Agriculture Experience Project. Your summer R.O.P. project can be either an ownership project which lasts throughout the duration of the summer, or a work experience project that is related to agriculture.

As a group, we will meet a total of 6 times throughout the summer, and will have a class project which includes the first annual Incoming Freshmen BBQ here at Pleasant Grove High School. The following are a list of meeting dates:

- Monday June $2^{\text {nd }}$

- Monday June $9^{\text {th }}$

- Monday June $16^{\text {th }}$

- July $7^{\text {th }}$

- Monday July $14^{\text {th }}$

- Wednesday July $23^{\text {rd }}$ (Incoming Freshmen BBQ)

Class meetings (all held on a Monday), will begin at 9:00am - during this time, you will have an opportunity to update your Record Book and plan for the Incoming Freshmen BBQ. As a group, the Summer R.O.P. class will plan the $B B Q$, including a flyer, activities, food, presentations, etc. to put on the best $B B Q$ possible. In order to be eligible to earn an " $A$ " in this class, you are required to attend at least 3 of the class meetings, and the Incoming Freshmen $B B Q$ in addition to turning in a recap for each of the 8 weeks the R.O.P. class runs.

Each week, including those we do not meet ( 8 weeks total), you are responsible for submitting, either in person or via email, a short (minimum 1 paragraph of 5 to 7 sentences) recap of what you did regarding your project for the week.

In order to enroll in Summer R.O.P., you must turn in the completed enrollment application by Thursday May $1^{\text {st }}$ to Ms. Forster.

Please let me know if you have any questions! 
Support Material 12:

- Graduate Follow Up

\section{Survey}




\section{Pleasant Grove High School Agriculture Department}

\section{Support Material 12: Graduate Follow Up Survey}

The Graduate Follow Up Survey is mailed to the addresses of all graduate students and made available via the online survey tool SurveyMonkey. These students are surveyed to allow for proper entry on the year's R-2 Roster, and allows us to keep track of our graduates and assists in completing American FFA Degree applications.

We hope to increase the number of results we receive by making the survey avaialable online for students to complete as this is sometimes a faster and easier process. 


\section{Pleasant Grove High School Agriculture Department Graduate Follow-Up}

Also available online through "Survey Monkey" at http://www.surveymonkey.com/s/9X9JLNB

Name:

Street Address:

City, State, Zip Code:

Phone:

Email:

Year of Graduation:

1. What are you doing at the present time?

_ Attending School

Working $\quad$ Full-time
Part-time
_ Not working
O Other

2. In what type of business or industry are you employed?

3. What is your job title or job description?

4. Which statement best applies to your present occupation?

I am using most of the skills I learned in the Ag program at PGHS.

- I am using some of the skills I learned in the Ag program at PGHS.

I am not using any of the skills I learned in the Ag program at PGHS.

5. What type of school are you currently attending?

_ Community/Junior College

_ 4-Year College/University

- Adult Education

_ Trade/Technical School

_ Private Business School

_Other:

6. What is your major course of study?

7. How would you rate the training you received in the PGHS Ag program?

_Excellent _ Good _ Fair _ Poor

8. How do you rate the career guidance and counseling you received in the Ag program? 



\section{Support Material 13:}

Graduate Follow Up Survey Results 


\section{Pleasant Grove High School Agriculture Department}

\section{Support Material 13: Graduate Follow Up Survey Results}

Results from our Graduate Follow Up Survey are entered into our R-2 Report. This allows us to keep an accurte record of where are graduates are and what they are doing, as well as provided feedback on the department as a whole. This year, we received all of our graduate survey results via the online survey tool SurveyMonkey, so while acutal surveys are not available, attached is the R-2 Report Data as well as the findings from the survey which are presented at our Fall Advisory Committee Meeting each year. 


\section{Pleasant Grove High School Agriculture Department \\ Graduate Follow Up Survey Results \\ * Represents the percentage of students who responded}

1. What are you doing at the present time?

90\% Attending School

$70 \%$ Full-time

20\% Part-time

0\% In the military

0\% Homemaker
20\% Working

$10 \%$ Full-time

$10 \%$ Part-time

0\% Not working

2. Employed in an Agriculture related industry? $31 \%$

3. Which statement best applies to your present occupation?

$60 \%$ I am using most of the skills I learned in the Ag program at PGHS.

$10 \%$ I am using some of the skills I learned in the Ag program at PGHS.

$30 \%$ I am not using any of the skills I learned in the Ag program at PGHS.

4. What type of school are you currently attending?

\begin{tabular}{|c|c|}
\hline $40 \%$ Community/Junior College & 0\% Trade/Technical School \\
\hline $50 \%$ 4-Year College/University & $0 \%$ Private Business School \\
\hline
\end{tabular}

5. Enrolled in an Agricultural major course of study? $\mathbf{5 0 \%}$

6. How would you rate the training you received in the PGHS Ag program?

$60 \%$ Excellent $10 \%$ Good $30 \%$ Fair $\quad 0 \%$ Poor

7. How do you rate the career guidance and counseling you received in the Ag program?

$70 \%$ Excellent $\quad 0 \%$ Good $30 \%$ Fair $\quad 0 \%$ Poor

8. Please check the following areas you feel are valuable components of FFA:

$67 \%$ Officer and committee chairperson experience

$88 \%$ Judging contests

$78 \%$ Advanced degree and proficiency awards

$78 \%$ Participation in chapter activities \& working with others

$100 \%$ Raising livestock, shows, fairs, etc.

$19 \%$ Other (please describe): Attending Conferences, Learning basics that can now be

applied to college classes

9. What were the most valuable aspects of the SAEP (supervised projects)?

$69 \%$ Learning skills related to future ag employment

$88 \%$ Development of responsibility

$63 \%$ Learning record keeping 
$19 \%$ Other (please describe): Reponses included "finding your passion", budgeting, and "raising a

outcome".

living animal from start to finish with a positive

10. Please rate the facilities and equipment used at PGHS for the Ag program:

Facilities:

0\% Overcrowded

$22 \%$ Adequate space

provided

$88 \%$ Modern

$0 \%$ Out-of-date

Equipment:

$77 \%$ Modern

$11 \%$ Well-maintained

$0 \%$

Out-of-date

$11 \%$

$0 \%$ Poorly maintained

$0 \%$ Other (please describe):

\section{Graduate Follow-up Report}

Filing Year $=2013$

\# CA0530 Elk Grove - Pleasant Grove

Pleasant Grove HS

9531 Bond Road

Elk Grove, CA 95624

Printed: 6/10/2014 5:22:08 PM

\begin{tabular}{|l|l|}
\hline Total Seniors (Year=2012 ): & 92 \\
\hline $\begin{array}{l}\text { Total Seniors having completed } \\
3 \text { or more years of Ag Instruction: }\end{array}$ & 35 \\
\hline \multicolumn{2}{|l|}{ Program Completer Status } \\
\hline Two Year College Ag Major & 3 \\
\hline Two Year College Non-Ag Major & 12 \\
\hline Four Year College Ag Major & 5 \\
\hline Four Year College Non-Ag Major & 5 \\
\hline Employed - Parttime Non-Ag Job & 1 \\
\hline Employed - Fulltime Ag Job & 2 \\
\hline Location or Position Unknown & 7 \\
\hline
\end{tabular}

Site developed and maintained by the California FFA As 


\section{Support Material 14:}

Comprehensive

Program Plan 


\section{Pleasant Grove High School Agriculture Department}

\section{Support Material 14: Comprehensive Program Plan}

Our Comprehensive Program Plan is in a large binder that holds all of the pertinent information relating to our agriculture program. It is updated every year, with the copy being provided to our Regional Supervisor, Jean Landeen.

Our Comprehensive Program Plan is in need of a major overhaul before our next Agriculture Incentive Grant Review with Hugh Mooney - hopefully the completion of this Master's Project will make that an easier process. 


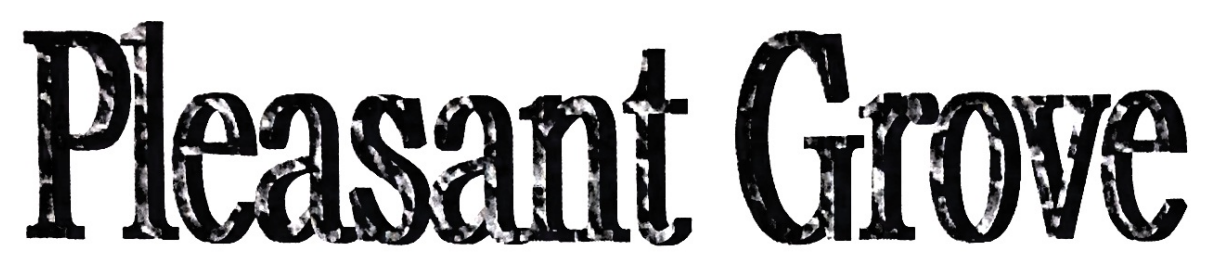

\section{Hign School}

Agriculure Dapartment

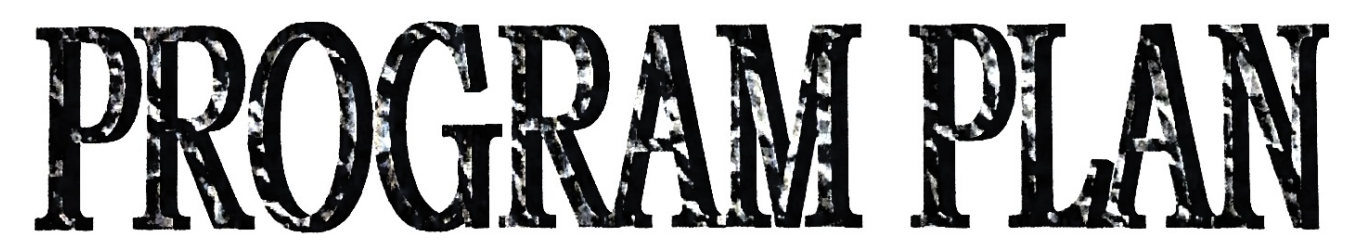


A. Job Market

B. Targeted Occupations

C. Total Program Goals \& Objectives

D. Program Description of Courses, SAEP \& Leadership

E. Program and Course Subject Matter Content Outline

F. Program Completion Standards

G. Description of Facilities \& Major Equipment

H. Five Year Facility \& Equipment Acquisition Schedule

I. Staff Assignments

J. FFA Program of Activities

K. School \& Department Policies

L. Proficiency Standards for Program Completers

M. Teacher Data Sheets

N. Roster of Agriculture Advisory Committee

O. Advisory Committee Minutes

P. Current Year Budget

Q. Signed Articulation Agreement \&/or Evidence of Articulation

R. Graduate Follow-Up System

S. List of Active Placement Sites

T. Recruitment Activities \& Materials

U. Staff In-Service Record

V. Staff Minutes

W. Department Inventory 


\section{JOB MARKET DESCRIPTION}

California is the leading state in agriculture. The Central Valley contributes greatly to this success. Production agriculture continues to be an important part of the economy of the United States. Due to advancements in technology, it has been possible for farmers to increase their production from fewer parcels of land. As a result, there has been an increase in employment in the service area of the industry.

Elk Grove is located in Sacramento County. The climate is variable can range from lows of 24-44 degrees to highs of $80-110$ degrees. Rainfall occurs from October through April with an average rainfall of 22 inches per year. The population size of Elk Grove in 2011 was 154,908 inhabitants.

Agriculture is an important part of Elk Grove's economy. Agriculture enterprises include grapes, dairy and beef cattle, and row crops. There is a strong demand for employment in high technology, professional service, commercial and retail enterprises. The top 10 agricultural commodities in Sacramento County for 2010 were: wine grapes, milk, Bartlett pears, nursery products, poultry, vegetables, corn (grain), cattle \& calves, fish, and alfalfa hay.

Elk Grove Unified School District is one of the fastest growing school districts in the country and is the fifth largest district in California. Elk Grove has experienced a huge population increase in the past 10 years. As a result, it has increased its number of schools such that currently there are nine high schools, four of which offer agricultural education classes. 


\section{TARGETED OCCUPATIONS}

Agriculture Production

Crop Production

Animal Production

Life Sciences

Agribusiness/Computers

Agribusiness

Omamental Horticulture

Floriculture $\underline{\text { Jobs }}$

Farmhand, Ranch Laborer, Feed Lot Hand, Field Crop Grower, General Maintenance

Livestock Handler, Farm Manager, Veterinary Technician, Pet Care, Ranch Laborer, Farm Hand

Ecologist, Biologist, Fish \& Game

Jobs

Ag Sales, Banking, Farm Accounting, Inventory Maintenance, Ag Secretary/Bookkeeper

$\underline{\text { Jobs }}$

Floral Marketing \& Design, Floral Sales, Floral Production \& Floral Delivery 


\section{PROGRAM GOALS \& OBJECTIVES}

- Upon completion of the agriculture education program, students will:

1. Be knowledgeable in world-wide agriculture and will make informed choices regarding postsecondary education choices.

2. Obtain skills necessary for success, including leadership (e.g. cooperation, teamwork, public speaking), problem-solving, and critical-thinking skills.

3. Be prepared to pursue further education or to enter a career after graduation from high school.

4. Set and achieve their goals in life.

\section{2-2013 Agriculture Department Goals}

1. Focus on Students

a)Increase collaborative learning within classes by including one group activity per week in each class period.

b) Highlight five individual student projects (e.g. in class activities, posters, etc.) per quarter in each of the Agriculture classrooms.

2. Increase Community Awareness

a)FFA Chapter participation in three (3) community service events during the year.

b) Publish one article in the local newspaper per academic quarter.

c) Publish a chapter newsletter once per academic semester.

3. Public Relations - increase Ag awareness with counselors/admin

a) $\mathrm{Ag}$ Teachers to hold a meeting with counseling department to share the Agriculture Department's goals and the student 4-year plan.

b)Email news articles and newsletters as published to Counselors, Administrators, Superintendent, School Board, Advisory Committee, Students, and Parents.

4. Maintain Rigor Within Curriculum

a)All media must have a direct tie to the curriculum and support the instructional program.

b)Follow all Expected Teacher Attributes for Pleasant Grove High School.

c)Assign one day per month to updating record books during class. 


\section{PROGRAM DESCRIPTION OF COURSES, SAEPS \& LEADERSHIP}

All courses within the Agricultural Education Program at Pleasant Grove High School incorporate the following 3 areas: classroom instruction, leadership development (FFA) and hands-on training (SAEP).

Classroom Instruction consists of lecture and laboratory settings. Students are assessed via assignments, quizzes, exams, lab reports, group projects, SAEPs, FFA participation and a comprehensive final exam.

Through their Supervised Agricultural Experience Programs (projects), students are able to apply the concepts and techniques learned in the classroom through a "real life" situation. Projects vary from an emphasis on animals, plants, or mechanics.

Students develop their leadership skills and abilities by participating in FFA activities. In the course "Applied General Science", we teach a FFA unit. In the subsequent courses, a brief unit on FFA is taught as a "refresher" for continuing students and an introduction for those students who are new to the program.

The following courses are offered at Pleasant Grove High School:

- Agricultural Biology

- Agricultural Leadership

- Animal Anatomy \& Physiology of Plants and Animals

- Applied General Science

- Economics in Agriculture

- Ornamental Horticulture (starting Fall 2013)

- Elements \& Principles of Floral Design 


\section{Program Completion Standards}

The Applied General Science and Agricultural Biology Courses are aligned with the CDE Science Content Standards. Common assessment is conducted with the science courses. All unit exams are submitted into the Prosper Assessment System. Each question is aligned with its specific standard(s). Instructors review the data analysis and compare with other instructors in their area to see which standards were met and which were not. If a student shows lack of progress, instructors develop intervention strategies based from reports.

Students are considered "program completers" if they are enrolled in an agriculture course for three years, including their senior year. It is assumed that upon completion of a course with a passing letter grade of "D" would indicate that the student has met the standards for the class.

In order for a student to be considered a "Program Completer" from the PGHS Agriculture Program, they must have been enrolled in an Agriculture class for three (3) years, including their senior year. Upon completion, students are recognized at graduation with a navy blue cord. Students, who earn their State FFA Degree, are recognized by the addition of the gold FFA sash at graduation. 


\section{Program Completion Standards}

The Applied General Science and Agricultural Biology Courses are aligned with the CDE Science Content Standards. Common assessment is conducted with the science courses. All unit exams are submitted into the Prosper Assessment System. Each question is aligned with its specific standard(s). Instructors review the data analysis and compare with other instructors in their area to see which standards were met and which were not. If a student shows lack of progress, instructors develop intervention strategies based from reports.

Students are considered "program completers" if they are enrolled in an agriculture course for three years, including their senior year. It is assumed that upon completion of a course with a passing letter grade of "D" would indicate that the student has met the standards for the class.

In order for a student to be considered a "Program Completer" from the PGHS Agriculture Program, they must have been enrolled in an Agriculture class for three (3) years, including their senior year. Upon completion, students are recognized at graduation with a navy blue cord. Students, who earn their State FFA Degree, are recognized by the addition of the gold FFA sash at graduation. 


\section{Description of Facilities}

The Pleasant Grove High School Agriculture Facility consists of:

- Classroom/Lab Building

- (2) laboratory science classrooms. Each with 8 lab stations, a teacher work station, 36 desks, 2 student computers, LCD projector, screen, phone, lockable cabinets, white board, sinks, eyewash station and document camera.

- Project Room: lockable cabinets, work table, 8 rolling chairs, copier (leased), network printer, phone, 2 closets, whiteboard, and tack board.

- Office: 3 teacher work stations, lockable cabinets, and 3 file cabinets.

- Large Animal Barn ( 7,392 square feet)

- Roofed structure to house market steers, goats, sheep \& swine. The north and south walls can be completely closed while the east and west walls are predominantly open. The east side of the barn is equipped to house swine on concrete flooring. The west side of the barn is equipped to house cattle, sheep and goats with concrete and dirt flooring. On either east/west side of the barn on the outside, there are exercise areas for the animals.

- The following items are within the barn: hand washing sink; wash rack area; phone; floral display cooler; storage cabinet.

- Small Animal Barn ( $\sim 528$ square feet)

- Roofed structure to house rabbits, chickens and turkeys. The north half of the building is for poultry on dirt floor and the south half is for rabbits on concrete floor.

- Rabbit area is fully enclosed with 1-2 foot mesh on the top and bottom for ventilation purposes. Rabbit cage structures are inside. Plus, a sink and spouts.

- The poultry area is wire fenced from the dirt to the roof.

- Floral Cooler ( 240 square feet)

- Greenhouse ( 1,980 square feet)

o In the final planning stage; to be approved by DSA in 2012-2013

- Open area on one end to provide a lecture setting with demonstrable table, overhead mirror, tables, and stools. Utility sink, phone, and rolling white board to be included.

- On the other end, there will be growing tables for plants. 


\section{PLEASANT GROVE HIGH SCHOOL AGRICULTURE DEPARTMENT \\ FIVE YEAR PLAN}

Year 1 2009-2010

1. Begin planning process of "Ag. Area"

2. Form Ag. Boosters Club

3. Repair equipment for County Fair projects.

4. Purchase lab equipment for Portable 6.

5. Organize tools/equipment in storage container.

6. Send "Graduate Follow Up" surveys and compile data to share with Ag Advisory Committee.

7. Purchase county fair equipment.

8. Purchase floral equipment and tools.

Year 2 2010-2011

1. Offer course "The Elements \& Principles of Floral Design"

2. Construction of "Ag. Area"

3. Purchase an Ag Department Vehicle

4. Form a Floriculture team.

Year 3 2011-2012

1. Opening of "Ag. Area".

2. Upgrade and replace student computers.

3. Landscape and plant trees on "Ag. Area".

4. Purchase tools for horticulture area.

5. Organize horticulture tools/equipment.

6. Offer Summer ROP.

Year 4 2012-2013

1. Offer Horticulture class.

2. Have planned plant sales.

3. Purchase tools for horticulture area.

4. Plant a variety of plants to use for identification purposes and for use in floral class.

Year 5 2013-2014

1. Begin pumpkin patch project with the FFA for sale in October.

2. Hire a part-time student manager.

3. Department repairs/replacements.

4. Hire Ag Department secretary. 


\section{PLEASANT GROVE HIGH SCHOOL AGRICULTURE DEPARTMENT \\ Student Eligibility, Leadership Development, \& SAEP Integration}

\section{Student Eligibility}

It is the policy at Pleasant Grove High School that all students participating in out-of-class activities, including FFA, maintain at least a 2.0 grade point average. The Agriculture Department supports this policy and applies it to all activities outside of class time. For activities during school hours, student must be cleared by his/her teachers prior to being out of class.

According to Pleasant Grove FFA's By-Laws, students willing to participate at Sacramento County Fair must maintain a 2.0 GPA and have a " $C$ " grade in their agriculture class. If not, student will not be allowed to enter their project(s) at the fair.

The PGHS Agriculture Department enforces the Elk Grove Unified School District Policy No. 6145 for ExtraCurricular Activities:

All students who wish to participate in extra-curricular activities must: 1) maintain a 2.0 average in their overall Elk Grove Unified School District 9-12 program during the quarter prior to their participation. During the participating season, a student who receives a failing progress report in any class will be removed from participation in the activity, be placed on academic probation, and will remain on probation until the grade returns to a passing status. 2) Meet standards of satisfactory citizenship. 3) Have a satisfactory attendance record.

In other words, a student with below a 2.0 GPA and/or an "F" in any of their classes at qualifying time (3rd Quarter) may not be eligible to exhibit or sell their animal at the Sacramento County Fair. This ruling would also include any other upcoming FFA activities.

\section{Leadership Development}

All students in the Pleasant Grove High School Agriculture Department are members of the FFA program. Students are required to attend at least two (2) FFA activities per quarter. Each activity is worth 50 leadership points, therefore, 100 points must be earned within this time frame. FFA participation is worth $10 \%$ of the student's grade in his/her agriculture class. If students participate in more than two activities, the points earned are extra credit to their current grade. Extra points cannot be "saved" and used for the following grading period. Activities that students may participate in are meetings, socials, judging teams, fairs/shows, leadership conferences, fundraisers, and other events as listed in the current year's FFA calendar of events. (See Program of Activities)

A 2-week unit regarding FFA organization will be taught in Applied General Science (gth grade). In subsequent courses, a brief overview of FFA will be taught for continuing students and new students to the program. Parliamentary Procedure will be part of the curriculum in Applied General Science.

\section{SAEP Integration}

All students in the Pleasant Grove High School Agriculture Department are required to have a SAEP project. The project may be related to any field of agriculture, such as production, processing, mechanics, business, nursery/landscaping, sales and service, etc. Students will be given a record book in order to maintain records on their project. The SAEP is worth $10 \%$ of the student's grade in his/her agriculture class. (See course syllabi). 


\section{PROFICIENCY STANDARDS FOR PROGRAM COMPLETERS}

a A program completer of the Pleasant Grove High School Agriculture Program is a student who has taken three years of coursework in Agriculture Science, including their senior year. Program completers will receive a blue cord and be recognized at the high school graduation ceremony. 
TEACHER DATA SHEET

Agricultural Education Instructors

Teacher: Izaskun Zallo

Credential Information:

Date lssued: $\quad 2001$

Credential: $\quad$ Professional Clear Single Subject Teaching Credential, Agriculture with CLAD emphasis

Date of Expiration: 2011

University: $\quad$ University of California, Davis

Date Issued: $\quad 2001$

Credential: $\quad$ Professional Clear Specialist Instruction Credential, Agriculture

Date of Expiration: $\quad 2011$

University: $\quad$ University of California, Davis

Work Experience:

2005-present Agriculture Instructor

Pleasant Grove High School

2004-2005 Teacher Resource Development Coordinator / Educator Specialist

Explorit Science Center

2001-2004 Agriculture Instructor

Ukiah High School

Summer $2001 \quad$ Chemistry Teacher

Davis Joint Unified School District

1998-1999 Post Graduate Researcher

University of California, Davis 


\section{TEACHER DATA SHEET}

Agricultural Education Instructors

Teacher: Maya O'Connor

Credential Information:

Date Issued:

Credential:

Date of Expiration:

University:

06/12/2008

Clear Specialist Instruction Credential (Agriculture)

07/01/2013

University of California, Davis

Date Issued:

Credential:

06/12/2008

Preliminary Single Subject Teaching Credential (Agriculture)

- With Supplemental/Subject Matter Authorization in Biological Sciences

Date of Expiration:

University:

07/01/2013

University of California, Davis

Work Experience:

2008-present

Agriculture Instructor

Pleasant Grove High School 


\section{PLEASANT GROVE HIGH SCHOOL AGRICULTURE DEPARTMENT \\ Advisory Committee}

\begin{tabular}{|c|c|c|c|c|c|c|}
\hline $\begin{array}{l}\text { First } \\
\text { Name } \\
\end{array}$ & $\begin{array}{l}\text { Last } \\
\text { Name } \\
\end{array}$ & Street Address & City & $\begin{array}{l}\text { Zip } \\
\text { Code }\end{array}$ & Phone Numbers & E-mail \\
\hline Gil & Albiani & 10221 Sheldon Road & Elk Grove & 95624 & $\begin{array}{l}682-2445 \text { (home) } \\
425-0330 \text { (cell) } \\
683-6655 \text { (work) }\end{array}$ & galbiani@golyon.com \\
\hline Dan & Forster & 13040 Orange Road & Wilton & 95693 & $\begin{array}{l}687-8988 \\
747-7766 \\
747-7080 \\
\end{array}$ & forster.d@frontiernet.net \\
\hline Betty & Hawes & 12659 Apricot Lane & Wilton & 95693 & $\begin{array}{l}687-7946 \\
217-7946 \\
\end{array}$ & $\begin{array}{l}\text { bettyhawes@citlink.net } \\
\text { bettyh@superiorfarms.com }\end{array}$ \\
\hline Tony & Jenovino & 9919 Jetmar Way & Elk Grove & 95624 & $\begin{array}{l}\text { 685-7461 (home) } \\
996-6681 \text { (cell) }\end{array}$ & Tjenovino@frontiernet.net \\
\hline Bill & Leicht & 9530 Magos Road & Wilton & 95693 & $\begin{array}{l}687-8546 \text { (home) } \\
718-2834 \text { (cell) } \\
\end{array}$ & LeichtwL@Lilly.com \\
\hline Howard & Lewis & $\begin{array}{l}\text { Cosumnes River College } \\
8401 \text { Center Parkway }\end{array}$ & Sacramento & 95823 & & LewisH@CRC.losrios.edu \\
\hline Lynn & Martindale & $\begin{array}{l}\text { UC Davis } \\
1 \text { Shield Ave. }\end{array}$ & Davis & 95616 & $606-7337$ & Imartindale@ucdavis.edu \\
\hline Hank & Meyer & 9531 Bond Road & Elk Grove & 95624 & $686-0230$ & hmeyer@egusd.net \\
\hline Christy & Moustris & 9510 Elk Grove-Florin Road & Elk Grove & 95624 & $686-7709 \times 7709$ & cmoustris@egusd.net \\
\hline Maya & O'Connor & 9531 Bond Road & Elk Grove & 95624 & $\begin{array}{l}686-0230 \times 4927 \text { (work) } \\
530-304-9380 \text { (cell) }\end{array}$ & moconnor@egusd.net \\
\hline $\mathrm{Jim}$ & Perham & 8135 Sloughhouse Road & Elk Grove & 95624 & $\begin{array}{l}425-9379 \text { (cell) } \\
682-7730 \text { (work) }\end{array}$ & perhami@aol.com \\
\hline Katie & Pierce & 9712 Mindy Lane & Wilton & 95693 & $687-7654$ & rktpierce@hotmail.com \\
\hline Izaskun & Zallo & 9531 Bond Road & Elk Grove & 95624 & $\begin{array}{l}686-0230 \times 4894 \text { (work) } \\
690-2883 \text { (cell) }\end{array}$ & izallo@egusd.net \\
\hline
\end{tabular}




\section{GRADUATE FOLLOW.UP SYSTEM}

The first time that the PGHS Agriculture program had program completers was in June 2008 with 18 students. In 2009, we had an additional 18 students complete the program.

In following up with the 2008 graduates, no surveys were sent. Past students were contacted either via a phone call or via email. Data collected was pertaining to the information needed for the R-2 and Graduate data input on www.calaged.org.

In 2009, a survey was created and sent out to program completers for 2008 and 2009 . A document was created to tabulate the results. Once information is gathered, results will be shared with $\mathrm{Ag}$ Advisory Committee and the principal. 


\title{
ACTIVE PLACEMENT SITES
}

\author{
Sheldon Feed \& Supply \\ Corky Donnelly \\ 8928 Grant Line Road \\ Elk Grove, CA 95624-1413 \\ 916-686-6400 \\ Student: Jordan Jenovino \\ Cluster Area: Animal Science, Plant \& Soil Science \& Ag Business \\ VanWarmerdam Dairy \\ Peter VanWarmerdam \\ 12121 McKenzie Road \\ Galt, CA 95632 \\ 209-914-4112 \\ Student: Melissa Withrow \\ Cluster Area: Animal Science
}




\section{RECRUITMENT ACTIVITIES}

The Pleasant Grove High School Agriculture Department conducts the following recruitment activities during the school year:

1. Visit $8^{\text {th }}$ grade science classes at Katherine L. Albiani Middle School (feeder school) in early Spring. FFA members present a power point (see attachment) and do team-building activities with students.

2. During National FFA Week, activities are planned to promote FFA and the agriculture courses.

3. Hold a "Farm Day" on-campus, in which during lunch, FFA members teach other students regarding their SAEP projects and explain what they learn in their agriculture courses.

4. Attend $9^{\text {th }}$ Grade Parent Orientation Meeting during early Spring.

5. Agriculture students, PGHS Administrators and Counseling Department receive a copy of the PG FFA Student Handbook. 
Student Name:

Student ID:

\section{Agricultural Biology}

Students are graded on their ability to accomplish or perform certain tasks as certified by instructor.

Rating Scale:
3 Excellent
2 Good
1 Basic
0 Does not meet basic standards
n/a Not Applicable

Competency Level:

1. Show relationship between biology and the industry of agriculture.

2. Describe the difference between living and nonliving things.

3. Identify molecules of life and explain their functions.

4. Examine cell structure and relate structure to function.

5. Compare and contrast photosynthesis and respiration.

6. Examine Mendel's Laws and explain relationships.

7. Compare and contrast differences between chromosomes and genes.

8. Generalize the relationship between meiosis and mitosis.

9. Analyze how gene technology is utilized in agriculture and medicine.

10. Demonstrate how energy flows through an ecosystem.

11. Identify the world's biomes.

12. Demonstrate an understanding of ecosystems and interactions within the environment.

13. Demonstrate an understanding of the environment and some of the problems faced by the world's ecosystems.

14. Utilize classification systems of living things.

15. Distinguish and differentiate the five kingdoms.

16. Demonstrate an understanding of plant science and advancements within the plant science field.

17. Demonstrate computer and software knowledge through research projects and technical writing.

18. Participate in various FFA and leadership activities.

19. Maintain ongoing supervised agricultural experience program (SAEP). 
Student Name:

Student ID:

\section{Economics in Agriculture}

Students are graded on their ability to accomplish or perform certain tasks as certified by instructor.

Rating Scale:

$\begin{array}{ll}3 & \text { Excellent } \\ 2 & \text { Good } \\ 1 & \text { Basic } \\ 0 & \text { Does not meet basic standards } \\ \text { n/a } & \text { Not Applicable }\end{array}$

Competency Level:

1. Define and use correctly economic terms and concepts.

2. Understand the laws of supply and demand discuss how they affect scarcity, price and quantity of products.

3. Explain foreign exchange and its effects on the US dollar.

4. Demonstrate wise credit use in their SAEP.

5. Describe the role of cooperatives in agriculture.

6. Explore various careers in agri-business.

7. Complete State FFA Degree Application.

8. Maintain good record-keeping skills.

9. Demonstrate computer and software knowledge through research projects and technical writing.

10. Participate in various FFA and leadership activities.

11. Maintain ongoing supervised agricultural experience program (SAEP).

Certifying Instructor

Course Grade

Date 
Student Name:

Student ID:

\section{Applied General Science}

Students are graded on their ability to accomplish or perform certain tasks as certified by instructor.

Rating Scale:

$\begin{array}{ll}3 & \text { Excellent } \\ 2 & \text { Good } \\ 1 & \text { Basic } \\ 0 & \text { Does not meet basic standards } \\ \text { n/a } & \text { Not Applicable }\end{array}$

Competency Level:

1. Develop knowledge and understanding of practical and essential Earth Science concepts and the principles Earth Science shares with other disciplines.

2. Understand basic principles of Earth Science and think from an Earth Science perspective

3. Develop an understanding of scientific inquiry.

4. Use science as a way to demonstrate the relevance of Earth Science to their life and environment.

5. Utilize local and regional issues and concerns to solve problems and foster a sense of Earth stewardship in their communities.

6. Understand main course topics of astronomy, weather, oceanography, plate tectonics, and earth forces.

7. Understand the nature, origin and distribution of Earth's energy and resources.

8. Integrate mathematics, language arts, and employability standards including creative thinking and problem solving skills, and technological literacy.

9. Participate in various FFA and leadership activities.

10. Develop an ongoing supervised agricultural experience project (SAEP). 
Student Name:

Student ID:

\section{Animal Anatomy and Physiology}

Students are graded on their ability to accomplish or perform certain tasks as certified by instructor.

Rating Scale:

3 Excellent

2 Good

1 Basic

0 Does not meet basic standards

n/a Not Applicable

Competency Level:

1. Explore a variety of systems that make animals unique from any other organism on our planet, with a focus on mammals that are most important to human culture as we know it.

2. Master a broad body of knowledge regarding anatomy and physiology, including scientific method, classification systems, mammalian production, production management, health care and diseases, genetics reproduction, nutrition, respiration/circulatory, digestion, nervous system, genetics, excretory system, endocrine system, muscle/skeletal system.

3. Effectively use analytical skills to implement the scientific method to solve problems.

4. Work effectively with others to solve problems and produce products.

5. Complete individual projects and long-term assignments, class discussions, labs and dissections.

6. Participate in various FFA and leadership activities.

7. Develop an ongoing supervised agricultural experience project (SAEP).

8. Use factual data to support an argument or position. 
Support Material 15:

Advisory Committee

Meeting Agendas 


\section{Pleasant Grove High School Agriculture Department}

\section{Support Material 15: Advisory Committee Meeting Agendas}

The Agriculture Advisory Agendas help guide the focus of our meetings. It is essenstial to use our time efficiently so that our community members are not having their time wasted. 


\title{
PLEASANT GROVE AGRICULTURE DEPARTMENT
}

9531 Bond Road, Elk Grove, CA 95624 916-686-0230

IzaskunZallo izallo@egusd.net Jessica Forster-jforster@egusd.net

Jenna Swenson -

iswenson@egusd.net

\author{
Agriculture Department Advisory Committee Meeting \\ AGENDA \\ January 22, 2014 \\ PGHS Agriculture Department
}

1. Introductions

2. Review \& Approve Minutes of previous meeting

3. Pathways Discussion

- Program Completers Defined

4. Course Enrollment, Graduate and Retention Numbers

5. Department Goals

6. Unfinished Business

7. Future Ag Advisory Committee Meeting Dates -

- January 22 $2^{\text {nd }} @ 6 \mathrm{pm}$

- May29 $@ 6 \mathrm{pm}$

8. Adjournment 


\section{PLEASANT GROVE AGRICULTURE DEPARTMENT}

9531 Bond Road, Elk Grove, CA $95624916-686-0230$

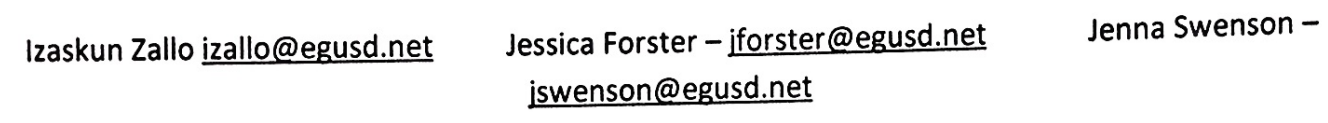

Agriculture Department Advisory Committee Meeting

AGENDA

Tuesday, June 10,2014@6:00 pm

PGHS Agriculture Department

1. Review \& Approve Minutes of previous meeting

2. Enrollment for $2013-2014$

3. Greenhouse Update

4. FFA Highlights - Spring 2014

5. Future Ag Advisory Committee Meeting Dates-

6. Unfinished Business

7. Adjournment 


\section{Support Material 16: Adviosry Committee Meeting Minutes}


Pleasant Grove High School Agriculture Department

\section{Support Material 16: Advisory Committee Meeting Minutes}

Our Agriculture Advisory Committee meets at least twice each year to discuss the direction of our progam, and making any modifications needs as the program continues to evolve and change.

Our second meeting will be held on June10th, so unforntunately minutes had not yet been prepared from this meeting, but I have included the minutes from our fall meeting this year. 
In attendance: Michelle Doiron, Dan Forster, Jessica Forster, Lynn Martindale, Angela Mayfield, Jenna Swenson, Izzy Zallo

The meeting was called to order at 6:14pm by Dan Forster at PGHS in the ag department.

1. Angela moved to accept the minutes from the previous meeting as read and Lynn seconded the motion. The motion passed by voice vote.

2. Sharing Ideas of Ag Program names

a. Zallo's Animal \& Anatomy classes had a class assignment to come up with a acronym/brand name for the ag department/program

b. Angela recommended that we look at other states for names of their academy programs.

c. It was suggested to possibly add science into the final program name.

3. Future of $\mathrm{Ag}$ Funding

a. Each student had an assignment in class to write to their local legislators and to find their representatives. They were sent to governor, senator and assembly member. Steinberg responded to students wrote to students telling them they should talk to their district about the funding.

b. Capitol Ag Day - discussion with cut flowers association to possibly make appointment with governor or office staff when floral arrangement is delivered.

c. Lynn suggested that as a group the ag high schools in the district should get together to propose a plan, set amount per student to ask for the local funding money that is allocated for career technical education.

4. Program Completer Defined -

a. A program completer of the PGHS Agriculture program is defined as a student who completes three (3) years, including their senior year in the program and completes coursework in one of the Career Pathways offered. These students will receive a blue cord to wear at graduation. Students who earn their State FFA Degree will wear the gold FFA sash. Students who complete four (4) years in the Agriculture program will receive a pin to wear for their achievement.

i. Advisory members agreed that this was a clear and acceptable definition.

5. Unfinished Business

a. 19 State FFA Degrees - New PG Record

b. Ag Department Survey -

i. Email Angela the survey to create on Survey Monkey

ii. Add a question clarification question about SAE's describing what category it fits in.

iii. For Grad survey - add question about earning FFA scholarship and able to get a job from being in ag class

c. Future Ag Meeting - May $29^{\text {th }} @ 6 p m$.

6. Meeting was adjourned at 8:14 pm. 
Support Material 17:

Advisory Committee

Constitution

\& By-Laws 
Pleasant Grove High School Agriculture Department

\section{Support Material 17: Advisory Committee Constitution \& By-Laws}

As Pleasant Grove High School is a fairly new school, begin opened in 2005, our Advisory Committee Constitution and By-Laws are fairly up to date. 
Pleasant Grove High School

Agriculture Advisory Committee

\section{BYLAWS}

\section{ARTICLE I - Name}

1. The name of this organization will be the Pleasant Grove High School Agriculture Advisory Committee, hereafter referred to as the Advisory Committee.

$$
\text { ARTICLE II-Purpose }
$$

Section A

1. To represent the community and advise the agriculture teachers and administration for achieving a successful agriculture program.

Section B

Understanding of Responsibility

1. The Advisory Committee is only advisory in character.

2. The advice is up to the teachers, school administrators, or school board as appropriate to accept or reject.

3. The Advisory Committee has no administrative or policy forming power.

\section{Section C}

Functions and Duties of Advisory Committee

1. Review existing programs, courses of study, facilities, and equipment.

2. Make recommendations to the agriculture program.

3. Assist the teachers in finding suitable work stations (internships, work-study, cooperative learning, partnerships) for students in both production agriculture and agri-industry occupations.

4. Help advocate support for legislation and appropriations.

5. Help the teachers develop a list of capable resource persons for use as speakers, and/or judges for both in-school and out-of-school tests and contests.

6. Help provide the teacher with technical assistance and keep him/her aware of new developments in the agricultural industry.

\section{ARTICLE III-Membership}

\section{Section A}

The Advisory Committee will consist of up to twelve people, made up of parents, community members, agricultural industry representatives, and 1-2 student representative(s).

1. Committee representatives will be selected by the agriculture instructors and approved by the school administration annually.

2. Non-voting school site members consist of all agriculture instructors and site administrator.

3. The committee will fill its own replacements.

\section{Section B}

Members:

1. Should be successful agriculturists and/or individuals engaged in a significantly related occupation.

2. Should have recent, successful, firsthand, and practical experience in the field of agriculture. 
3. Should exhibit substantial interest in the agriculture program.

4. Should be sought as public-spirited individuals who understand a specialized area and are willing to contribute their knowledge and advice as a member of a cooperative, constructive group.

5. Should recognize the time required and express a willingness to serve on the committee.

\section{ARTICLE IV - Voting}

1. Each representative who is present at a meeting will have one vote on motions or actions that come before the Advisory Committee.

2. Twenty-five percent of the membership shall constitute a quorum.

3. Business is transacted by a simple majority vote.

\section{ARTICLE V-Officers}

\section{Section A}

1. The officers of the Advisory Committee shall be the chairperson, vice-chairperson, and recorder.

2. Chairperson and vice-chairperson should be a voting member elected by the committee.

3. Officers will be elected at the beginning of each school year. Officers shall advance through the chairs.

4. An agriculture teacher will serve as recorder and general interpreter.

\section{Section B}

Officer duties

Chairperson:

1. Presides over all meetings.

2. Arranges and organizes meetings with the Agriculture Department Head.

3. Establishes subcommittees as needed.

\section{Vice-Chairperson:}

1. Assists chairperson as needed.

2. Oversees chairpersons' duties in their absence.

\section{Recorder:}

1. Records minutes and distribute them to all voting and non-voting committee members, the school principal and the vocational education director.

\section{ARTICLE VI - Meetings}

1. The Advisory Committee will meet a minimum of twice per year.

2. Necessity should always determine the exact number. 
Support Material 18:

Proficiency Standards 


\section{Pleasant Grove High School Agriculture Department}

\section{- Support Material 18: Proficiency Standards}

Students must meet the minimum Proficiency Standards to be successful in any agriculture programs.

Currently, we only offer one ROP class: Agriculture Sales, Service, and Animal Science. This class is offered each summer, and is devised for students with year round SAE projects. The Proficiency Standards for this class have been developed through the Sacramento County Regional Occupational Program. Students are awarded a certificate of completion at the conclusion of earch year's ROP class. 
Student Name:

Student ID:

\section{ROP Sales, Service and Animal Science}

Students are graded on their ability to accomplish or perform certain tasks as certified by instructor.

Rating Scale:

$4 \quad$ Excellent

4 Good

3 Basic

2 Does not meet basic standards

n/a Not Applicable

Competency Level:

1. Define and use correctly agricultural terms as it relates to the sales and service industry

2. Understand the laws of supply and demand as well as price cycles as it relates to agriculture commodities

3. Demonstrate proper customer service techniques through phone and email methods

4. Demonstrate wise credit use in their SAE project

5. Demonstrate current marketing techniques within their SAE Project

6. Explore various careers within the agriculture industry

7. Complete and maintain an up-to-date California Agriculture Education Record Book

8. Maintain good record-keeping skills both in a Record Book and through business records

9. Maintain ongoing supervised agricultural experience program (SAE)

10. Participate in various FFA and leadership activities 
ค

Support Material 19:

- Teaching Credentials 


\section{Pleasant Grove High School Agriculture Department}

\section{Support Material 19: Teaching Credentials}

I hold the following cleared California Teaching Credentials:

- Specialist Instruction Credential in Agriculture Issued 2010

- Single Subject Teaching Credential in Agriculture Issued 2013

Each credential must be renewed with the California Department of Teacher Credentialing every five years. 


\section{C.sov \\ COMMISSION ON \\ TEACHER CREDENTIALING \\ Home | FAQ | Glos \\ Ait Agency User [ Search \\ Ensuring Ectucator Excellence}

Document Number:101078034 >

\section{Back}

To view the educator's public records (current documents, all documents held and Adverse and Commission Actions), click on the Educator's Last Name.

Educator Information:

Last Name: FORSTER

First Name: JESSICA

Middle Name:LYNN

\section{Document Information:}

Document Number: 101078034

Document Title:Specialist Instruction Credential (Agriculture)

Term:Clear

Status: Valid

Issue Date:2/1/2010

Expiration Date:3/1/2015

Original Issue Date: $2 / 1 / 2010$

Grade:

Special Grade:

SB1969 (Title 5 §80487):

\section{A uthorization / Subjects}
Authorization $\triangle$
Code
Authorization Description $\Delta$
Subject $\triangle$ Sut
This credential authorizes the holder to teach agriculture in grades twelve and below, including preschool, and in classes organized
R3A1 primarily for adults. It also authorizes the holder to develop and coordinate curriculum, develop programs, and deliver staff
Code $\nabla$ De: development for agriculture education programs coordinated by
AGRI
Agri school districts or county offices of education.

\section{Renewal Requirements}

Please disregard any \# signs you may see below and refer to the "Additional Description" column to the right fo

Renewal
Code

The term of this credential is limited by the term of the prerequisite credential. To renew this cre

Employment Restrictions 
Agency User/Search

\title{
Back
}

To view the educator's public records (current documents, all documents held and Adverse and Commission Actions), click on the Educator's Last Name.

\section{Educator Information:}

\author{
Last Name:FORSTER \\ First Name: JESSICA \\ Middle Name: LYNN
}

\section{Document Information:}

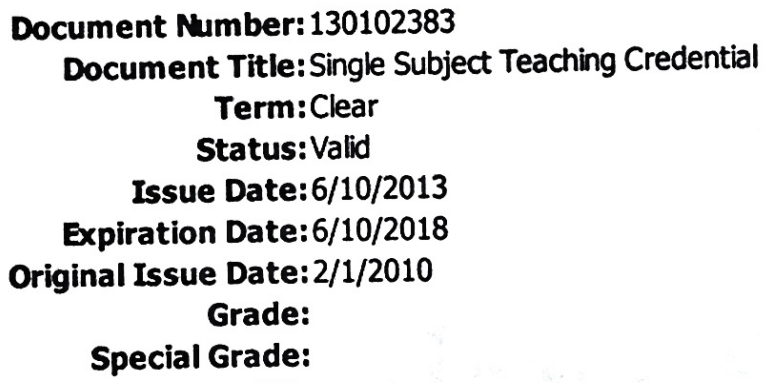

\section{Authorization / Subjects \\ Authorization
Code \\ The following instructional services may be provided to English learners: (1) instruction for English language development in grades twelve and below, including preschool, and in classes organized primarily for adults. If the prerequisite credential or permit is a designated subjects adult education teaching credential, a child development instructional permit, or a child development supervision \\ ELA1 permit, English language development instruction is limited to the programs authorized by that credential or permit; (2) specially designed content instruction delivered in English in the subjects, programs and at the grade levels authorized by the prerequisite credential or permit. This English learner authorization also covers classes authorized by other valid, non-emergency credentials or permits held, as specified in Education Code Section 44253.3. listed in grades twelve and below, including preschool, and in classes organized primarily for adults.}

\section{Renewal Requirements}

Please disregard any \# signs you may see below and refer to the "Additional Description" colum to the righi 1

\begin{tabular}{l|l} 
Renewal & $\Delta$ \\
Code & Renewal Description $\Delta$
\end{tabular}

R2O

To renew this credential, the holder needs to submit only an application and fee to the Comm months before the expiration date. The renewal period is five years. 
Support Material 20:

- Calendar of Activities 


\section{Pleasant Grove High School Agriculture Department}

\section{Support Material 20: Calendar of Activities}

Every year, a list of all FFA Activities is compiled for our local FFA Chapter. This list is provided to all students and parents at the beginning of each school year, and is also available throughout the year via the Agriculture Department's webpage. By distributing the FFA Activities list at the beginning of the school year, students are able to plan ahead so that they are able to attend their two required FFA activities each academic quarter. 
2013-2014

PLEASANT GROVE FFA CALENDAR

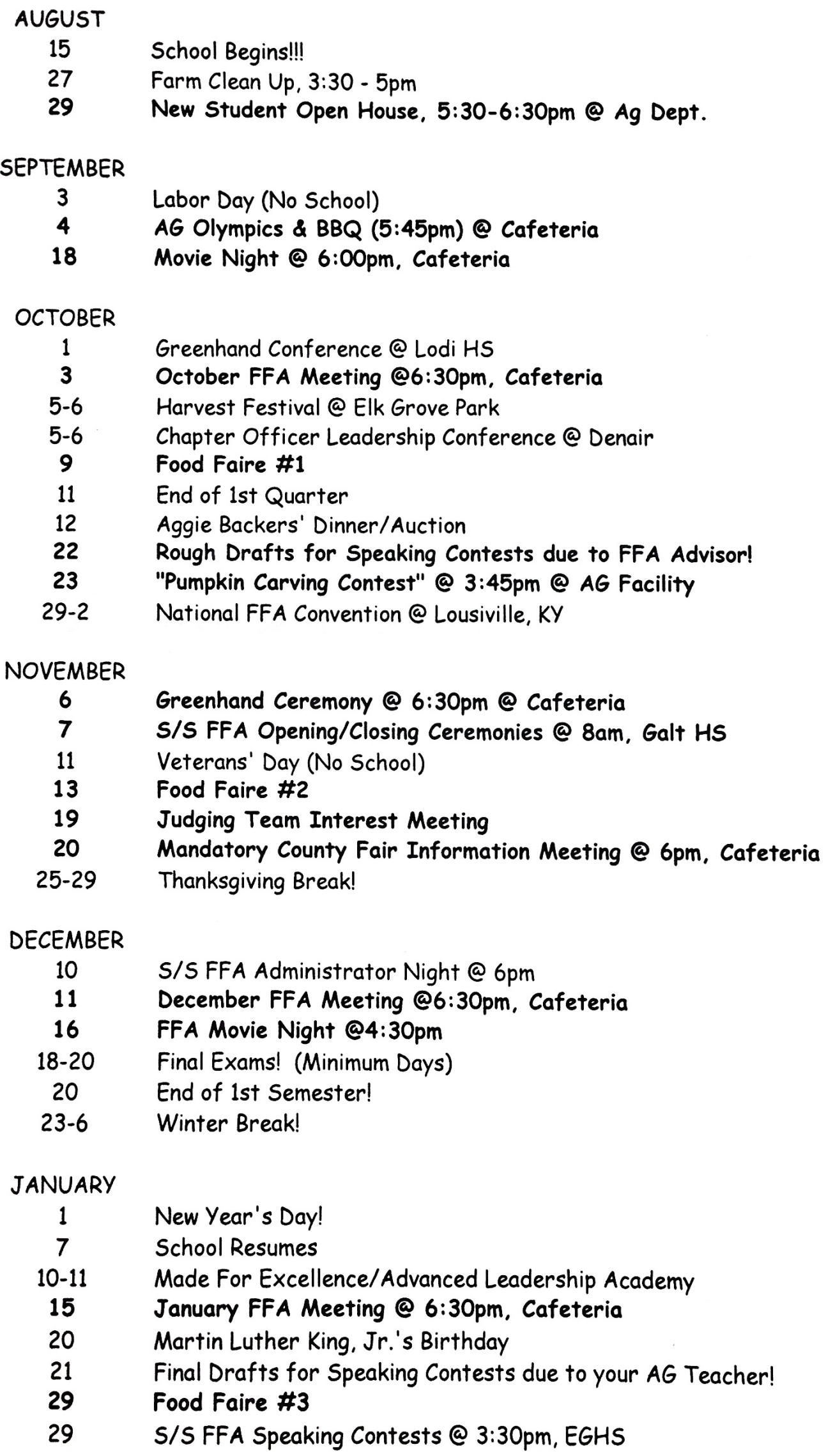


FEBRUARY

$4 \quad$ Central Region Proficiency Award Scoring @ Ripon HS @ 4pm

5 "Feel The Lovel" @ 6:30pm @ Cafeteria

10 Central FFA Region Officer Interviews @ Modesto JC

10 Lincoln's Birthday (No School)

12 Central Region FFA Speaking Contests Pre-lims @ Galt HS

16-22 National FFA Week!

17 Presidents' Day (No School)

18 Donkey Basketball Game vs. Elk Grove FFA

22 Central CATA/FFA Spring Regional Meeting @ Modesto JC @ 8am

$\begin{array}{cl}\text { MARCH } & \\ 1 & \text { CSU Chico Field Day } \\ 3 & \text { State Degree Ceremony. Sheldon HS } \\ 5 & \text { March FFA Meeting @ 6:30pm. Cafeteria } \\ 4-7 & \text { Sacramento Leadership Experience Conference } \\ 8 & \text { UC Davis Field Day } \\ 14 & \text { End of 3rd Quarter (Minimum Day Schedule) } \\ 15 & \text { Merced College Field Day } \\ 19 & \text { FFA Social @ 3:45pm, Cafeteria } \\ 20 & \text { Ag Day at the Capitol } \\ 29 & \text { Modesto JC Field Day }\end{array}$

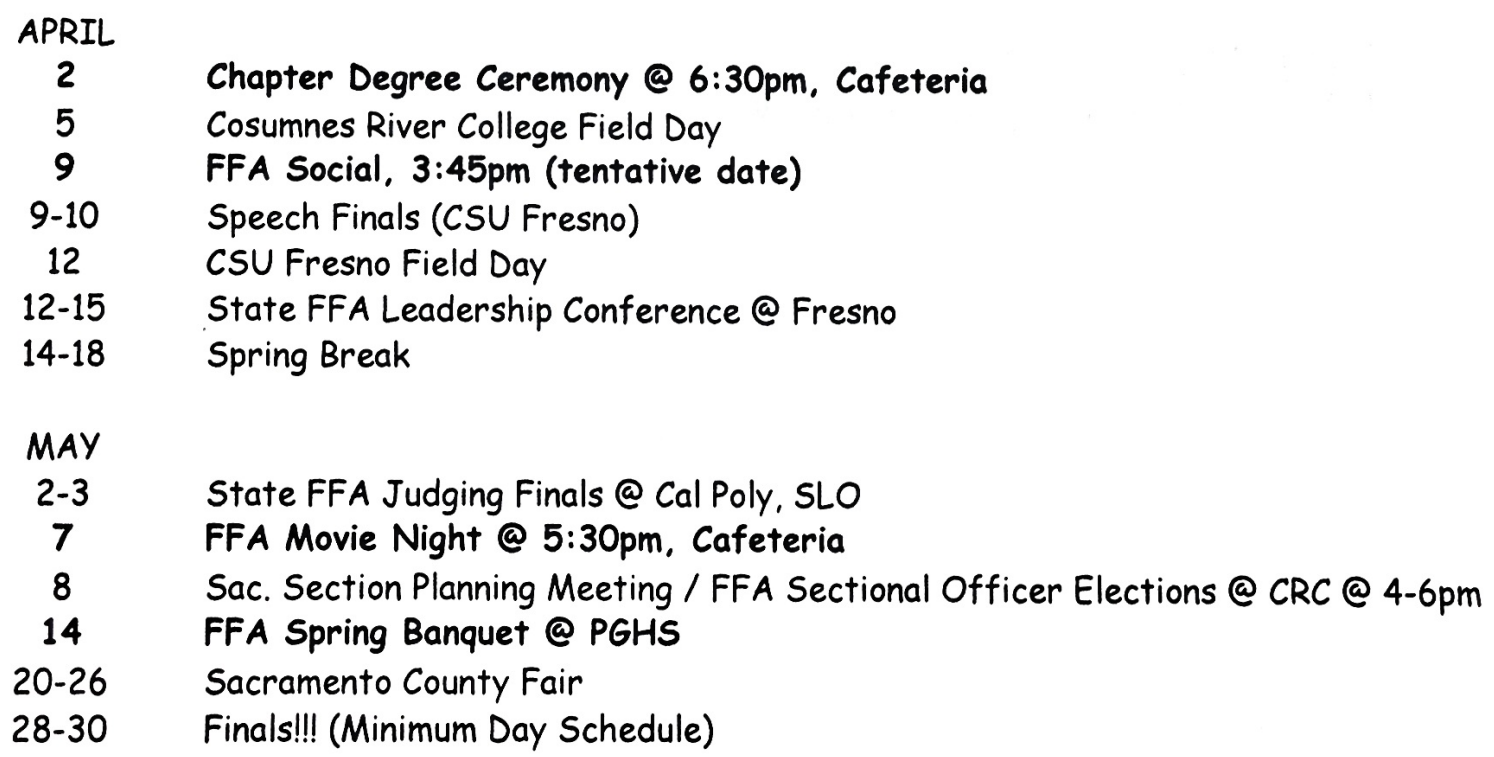

* Items on the calendar may change during the school year.

Check with your ag. instructor for updates! 
Support Material 21:

Professional Growth

and Development 


\section{Pleasant Grove High School Agriculture Department}

\section{Support Material 21: Professional Growth \& Development}

Attached is alist of the Professional Growth and Development Activities I attended throughour the 2013-2014 school year through our school, the California Agriculture Teachers Association, and the Junior Livestock Auction Committee. 


\begin{tabular}{|c|c|c|}
\hline Date & Activity Description & Location \\
\hline August 12,2013 & Staff Inservice & PGHS \\
\hline August 13, 2013 & Staff Meeting and Team Collaboration & PGHS \\
\hline August 14,2013 & District Inservice & District Office, EG \\
\hline September 10, 2013 & Junior Livestock Auction Committee Meeting (JLA) & EGHS \\
\hline September 12,2013 & Sectional CATA Meeting & EGHS \\
\hline October 6, 2013 & Sectional CATA Meeting @ COLC & Denair HS \\
\hline October 8,2013 & JLA Meeting & EGHS \\
\hline October 10,2013 & Staff Meeting & PGHS \\
\hline October 24,2013 & Earth Science Team Collaboration & PGHS \\
\hline November 12, 2013 & JLA Meeting & PGHS \\
\hline November 14, 2013 & Visual/Performing Arts (VAPA) Team Collaboration & PGHS \\
\hline November 23,2013 & Fall Regional CATA Meeting & MJC \\
\hline December 5, 2013 & Staff Meeting & PGHS \\
\hline December 10, 2013 & \begin{tabular}{|l|} 
Sectional CATA Administrators Night \\
\end{tabular} & EG Country Club \\
\hline December 12,2013 & State Degree App Committee Meeting & State FFA Center \\
\hline January 6, 2014 & Staff Inservice & PGHS \\
\hline January 9, 2014 & Staff Meeting & PGHS \\
\hline \begin{tabular}{|l|} 
January 14, 2014 \\
\end{tabular} & JLA Meeting & EGHS \\
\hline \begin{tabular}{|l|} 
January 16,2014 \\
\end{tabular} & Earth Science Team Collaboration & PGHS \\
\hline February 4, 2014 & Proficiency Scoring & Ripon HS \\
\hline February 11,2014 & JLA Meeting & EGHS \\
\hline February 6, 2014 & VAPA Team Collaboration & PGHS \\
\hline February 13, 2014 & Staff Meeting & PGHS \\
\hline February 22,2013 & Spring Regional CATA Meeting & PGHS \\
\hline February 27, 2014 & State Proficiency Scoring & State FFA Center \\
\hline March 6, 2013 & \begin{tabular}{|l|} 
Staff Meeting \\
\end{tabular} & PGHS \\
\hline March 11, 2014 & JLA Meeting & EGHS \\
\hline March 20, 2014 & Earth Science Team Collaboration & PGHS \\
\hline April 4, 2014 & WASC Committee Meeting & PGHS \\
\hline April 10, 2014 & \begin{tabular}{|l|} 
Staff Meeting \\
\end{tabular} & PGHS \\
\hline April 13, 2014 & State Degree App Committee Meeting & Fresno \\
\hline April 15,2014 & \begin{tabular}{|l|} 
JLA Meeting \\
\end{tabular} & EGHS \\
\hline April 29, 2014 & WASC Committee Meeting & PGHS \\
\hline May 8, 2014 & Staff Meeting & PGHS \\
\hline May 8, 2014 & Sectional CATA Meeting & EGHS \\
\hline May 13,2014 & JLA Meeting & EGHS \\
\hline May 15,2014 & VAPA Team Collaboration Meeting & PGHS \\
\hline May 23,2014 & \begin{tabular}{|l|} 
JLA Meeting \\
\end{tabular} & Cal Expo, Sacto. \\
\hline June 4, 2014 & Staff Meeting & PGHS \\
\hline June 10,2014 & JLA Meeting & EGHS \\
\hline June $22-26,2014$ & Summer CATA Conference & Cal Poly, SLO \\
\hline
\end{tabular}

*Department Meetings every Tuesday either before school or during lunch 
Support Material 22: R-2 Report 


\section{Pleasant Grove High School Agriculture Department}

\section{Support Material 22: R-2 Report}

The R-2 Report is updated in September of each school year with current student information, obtained through Student Data Sheets, to give our department an accurate report about the students and classes in our program. 
Pleasant Grove HS R2 Student Report

Year:2013

Gender

\begin{tabular}{|c|l|c|c|}
\hline Schnum & ProgName & Male & Female \\
\hline 467 & Ag Bus Mgt & 0 & 4 \\
\hline 467 & Ag Mech. & 2 & 0 \\
\hline 467 & Agriscience & 97 & 127 \\
\hline 467 & An. Science & 28 & 41 \\
\hline 467 & Forestry/NR & 1 & 0 \\
\hline 467 & O.H. & 28 & 93 \\
\hline 467 & Plant/Soil Sci. & 3 & 10 \\
\hline
\end{tabular}

Hispanic

\begin{tabular}{|c|c|c|}
\hline ProgName & Hispanic & Non-Hispanic \\
\hline Ag Bus Mgt & 0 & 4 \\
\hline Ag Mech. & 1 & 1 \\
\hline Agriscience & 49 & 175 \\
\hline An. Science & 16 & 53 \\
\hline Forestry/NR & 0 & 1 \\
\hline O.H. & 23 & 98 \\
\hline Plant/Soil Sci. & 5 & 8 \\
\hline
\end{tabular}

Race*
\begin{tabular}{|c|c|c|c|c|c|c|c|}
\hline ProgName & White & Black & Hispanic & $\begin{array}{c}\text { Americian } \\
\text { Indian }\end{array}$ & Asian & $\begin{array}{c}\text { Native } \\
\text { Hawaiian/Pacifc } \\
\text { Island }\end{array}$ & $\begin{array}{c}2 \text { or } \\
\text { more }\end{array}$ \\
\hline Ag Bus Mgt & 3 & 0 & 0 & 0 & 0 & 0 & 1 \\
\hline Ag Mech. & 2 & 0 & 0 & 0 & 0 & 0 & 0 \\
\hline Agriscience & 151 & 9 & 0 & 4 & 4 & 4 & 37 \\
\hline An. Science & 48 & 4 & 0 & 2 & 2 & 3 & 7 \\
\hline
\end{tabular}




\begin{tabular}{|c|c|c|c|c|c|c|c|}
\hline Forestry/NR & 1 & 0 & 0 & 0 & 0 & 0 & 0 \\
\hline O.H. & 75 & 15 & 0 & 1 & 1 & 4 & 15 \\
\hline $\begin{array}{c}\text { Plant/Soil } \\
\text { Sci. }\end{array}$ & 7 & 1 & 0 & 0 & 0 & 1 & 4 \\
\hline
\end{tabular}

\begin{tabular}{|c|c|c|c|c|c|c|c|c|c|}
\hline $\begin{array}{c}\text { Year } \\
\text { In } \\
\mathrm{Ag}\end{array}$ & Grade9 & Grade10 & Grade11 & Grade12 & Grade 13 & Grade14 & Grade 15 & Grade 16 & Total \\
\hline 1 & 134 & 17 & 45 & 36 & 0 & 0 & 0 & 0 & 232 \\
\hline 2 & 0 & 81 & 9 & 4 & 0 & 0 & 0 & 0 & 94 \\
\hline 3 & 0 & 0 & 74 & 7 & 0 & 0 & 0 & 0 & 81 \\
\hline 4 & 0 & 0 & 0 & 18 & 0 & 0 & 0 & 0 & 18 \\
\hline 5 & 0 & 0 & 0 & 0 & 5 & 0 & 0 & 0 & 5 \\
\hline 6 & 0 & 0 & 0 & 0 & 0 & 2 & 0 & 0 & 2 \\
\hline 7 & 0 & 0 & 0 & 0 & 0 & 0 & 2 & 0 & 2 \\
\hline Total & 134 & 98 & 128 & 65 & 5 & 2 & 2 & 0 & 434 \\
\hline \multicolumn{9}{|c|}{ Total 9-12 } & 425 \\
\hline
\end{tabular}

\section{Freshman Persistance:}

Cohort Year: 2010-2011

\begin{tabular}{|c|r|r|}
\hline Years in Ag Completed & Count & Percent \\
\cline { 1 - 2 } & 38 & $50 \%$ \\
\hline 2 & 10 & $13 \%$ \\
\hline 3 & 10 & $13 \%$ \\
\hline 4 & 18 & $24 \%$ \\
\hline Freshman Cohort Students & 76 & \multicolumn{1}{|c|}{} \\
\cline { 1 - 2 } Average Years Completed & 2.1 & \multicolumn{1}{|c}{} \\
\cline { 1 - 2 } & &
\end{tabular}


Data for Year: 2013-2014

\section{School:}

\# CA0530 Elk Grove - Pleasant Grove

Pleasant Grove HS

9531 Bond Road

Elk Grove, CA 95624

Teachers: 3

\section{Courses Offered:}

Type

\begin{tabular}{cc} 
Ag Biology & Agricultural Biology \\
Ag Biology & Agricultural Biology \\
Ag Biology & Agricultural Biology \\
Agriscience I & Applied General Science \\
Agriscience I & Applied General Science \\
Agriscience I & Applied General Science \\
Agriscience I & Applied General Science \\
Animal Science & Animal Anatomy \& Physiology \\
Animal Science & Animal Anatomy \& Physiology \\
\hline O.H./Floral & Elements and Principles of Floral Design \\
O.H./Floral & Elements and Principles of Floral Design \\
\hline O.H./Floral & Elements and Principles of Floral Design \\
O.H./Floral & Elements and Principles of Floral Design \\
Other Ag & Agricultural Science \\
\hline & TOTAL
\end{tabular}

Average Class Size
Enrollment H.S. Grad Credit

33 Life Science

36 Life Science

35 Life Science

32 Physical/Earth Sci.

27 Physical/Earth Sci.

31 Physical/Earth Sci.

36 Physical/Earth Sci.

$36 \quad$ Life Science

33 Life Science

$30 \quad$ Fine Arts

$34 \quad$ Fine Arts

$31 \quad$ Fine Arts

$36 \quad$ Fine Arts

28 Does Not Meet

458

32.7

\section{FFA Students by Pathway:}

$\begin{array}{lr}\text { Pathway } & \text { Count } \\ \text { Ag Bus Mgt } & 4 \\ \text { Ag Mech. } & 2\end{array}$




$\begin{array}{lr}\text { Agriscience } & 224 \\ \text { An. Science } & 69 \\ \text { Forestry/NR } & 1 \\ \text { O.H. } & 121 \\ \text { Plant/Soil Sci. } & 13 \\ & 434\end{array}$

FFA Students by Grade Level:

Grade Level Count

$\begin{array}{rr}9 & 134 \\ 10 & 98 \\ 11 & 128 \\ 12 & 65 \\ 13 & 5 \\ 14 & 2 \\ 15 & 2 \\ \text { Total } & 434\end{array}$

FFA Students by Years in Ag:

Years in $\mathrm{Ag}$ Count

$\begin{array}{cr}1 & 232 \\ 2 & 94 \\ 3 & 81 \\ 4 & 18 \\ 5 & 5 \\ 6 & 2 \\ 7 & 2 \\ \text { Total } & 434 \\ \text { Average Years } & 1.8\end{array}$


Freshman Persistance:

Cohort Year: 2010-2011

Years in Ag Completed Count Percent

$\begin{array}{ccc}1 & 38 & 50 \% \\ 2 & 10 & 13 \% \\ 3 & 10 & 13 \% \\ 4 & 18 & 24 \% \\ \text { Freshman Cohort Students } & 76 & \\ \text { Average Years Completed } & 2.1 & \end{array}$

R2 Teacher Information Pleasant Grove HS, Elk Grove Year: 2013

\begin{tabular}{|c|c|c|c|c|c|c|c|c|c|}
\hline $\begin{array}{l}\text { First } \\
\text { Name }\end{array}$ & Gender & $\begin{array}{c}\text { Ethnicit } \\
y\end{array}$ & $\begin{array}{l}\text { Total } \\
\text { Years } \\
\text { Teachin } \\
\text { g Ag. }\end{array}$ & $\begin{array}{c}\text { Credential } \\
\text { Type }\end{array}$ & $\begin{array}{c}9- \\
\text { Mont } \\
\mathrm{h} \\
\text { Salary }\end{array}$ & $\begin{array}{l}\text { Extende } \\
d \\
\text { Contract } \\
\text { Stipend }\end{array}$ & $\begin{array}{c}\text { FFA } \\
\text { Stipen } \\
\mathrm{d}\end{array}$ & $\begin{array}{c}\text { Departme } \\
\text { nt Head } \\
\text { Stipend }\end{array}$ & $\begin{array}{l}\text { SOE } \\
\text { Perio } \\
d\end{array}$ \\
\hline \begin{tabular}{l|l} 
Jessica & $\mathrm{L}$
\end{tabular} & Female & White & 4 & $\begin{array}{c}\text { Agricultur } \\
\mathrm{e} \\
\text { Specialist }\end{array}$ & 43099 & 0 & 0 & 0 & $\mathrm{~N}$ \\
\hline Izaskun & Female & Hispanic & 12 & $\begin{array}{c}\text { Agricultur } \\
\mathrm{e} \\
\text { Specialist }\end{array}$ & 67089 & 0 & 0 & 2726 & $Y$ \\
\hline \begin{tabular}{l|l} 
Jenna & $M$
\end{tabular} & 1 Female & White & 3 & $\begin{array}{c}\text { Agricultur } \\
\mathrm{e} \\
\text { Specialist }\end{array}$ & 45842 & 0 & 0 & 0 & $\mathrm{~N}$ \\
\hline \multicolumn{10}{|c|}{ Forster, Jessica } \\
\hline e Period & \multicolumn{2}{|c|}{ Beginning Time } & \multicolumn{4}{|c|}{ Course Title } & Enrollment & \multicolumn{2}{|c|}{ Type } \\
\hline 1 & \multicolumn{2}{|c|}{0800} & \multicolumn{4}{|c|}{ Applied General Science } & 32 & \multicolumn{2}{|c|}{ Agriscience I } \\
\hline 2 & \multicolumn{2}{|c|}{0906} & \multicolumn{4}{|c|}{ Elements and Principles of Floral Design } & 34 & \multicolumn{2}{|c|}{ O.H./Floral } \\
\hline 3 & \multicolumn{2}{|c|}{1012} & \multicolumn{4}{|c|}{ Elements and Principles of Floral Design } & 31 & \multicolumn{2}{|c|}{ O.H./Floral } \\
\hline 4 & \multicolumn{2}{|c|}{1118} & \multicolumn{4}{|c|}{ Elements and Principles of Floral Design } & 30 & \multicolumn{2}{|c|}{ O.H./Floral } \\
\hline 6 & \multicolumn{2}{|c|}{0105} & \multicolumn{4}{|c|}{ Elements and Principles of Floral Design } & 36 & \multicolumn{2}{|c|}{ O.H./Floral } \\
\hline
\end{tabular}




\begin{tabular}{|l|l|l|l|l|l|}
\hline 1 & 7 & 0211 & Prep & 1 & Prep \\
\hline
\end{tabular}

\begin{tabular}{|c|c|c|c|c|c|}
\hline \multicolumn{6}{|c|}{ Swenson, Jenna } \\
\hline Schedule & Period & Beginning Time & Course Title & Enrollment & Type \\
\hline 1 & 1 & 08:05 & Agricultural Science & 28 & Other Ag \\
\hline 1 & 2 & 9:06 & Agricultural Biology & 33 & Ag Biology \\
\hline 1 & 3 & $10: 12$ & Prep & 0 & Prep \\
\hline 1 & 4 & $11: 18$ & Applied General Science & 27 & Agriscience I \\
\hline 1 & 6 & $1: 05$ & Applied General Science & 36 & Agriscience I \\
\hline 1 & 7 & $2: 11$ & Applied General Science & 31 & Agriscience I \\
\hline
\end{tabular}

\begin{tabular}{|c|c|c|c|c|c|}
\hline \multicolumn{7}{|c|}{ Zallo, Izaskun } & Enrollment & Type \\
\hline Schedule & Period & Beginning Time & Course Title & 36 & Animal Science \\
\hline 1 & 1 & 0800 & Animal Anatomy \& Physiology & 33 & Animal Science \\
\hline 1 & 2 & 0906 & Animal Anatomy \& Physiology & 35 & Ag Biology \\
\hline 1 & 3 & 1012 & Agricultural Biology & 0 & Prep \\
\hline 1 & 4 & 1118 & Prep & 0 & SAE \\
\hline 1 & 7 & 105 & Project Supervision & 36 & Ag Biology \\
\hline
\end{tabular}


Support Material 23: Travel Request 
Pleasant Grove High School Agriculture Department Support Material 23: Travel Request

Attached is copy of a completed Travel Request per our district's procedure 
Use of a pay voucher form is allowed when:

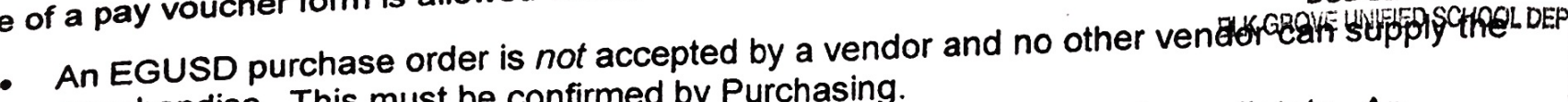
merchandise. This must be confirmed by Purchasing.

- An unexpected emergency required an employee to purchase items immediately. An explanation for the purchase is required.

- An EGUSD school site is requesting reimbursement. This would primarily be reimbursing the secondary ASB accounts or the elementary school site checking accounts.

All other requests for purchase of supplies, equipment or services should be submitted through the QSS PR Document Routing System.

Requests for expenses related to travel must be submitted on a Revolving Check Request form. Payable To:

\begin{tabular}{l|l|}
\hline Jessica Forster & For Accounting use Only: \\
Name & Vendor \# \\
\hline Address & $P V \#$ \\
\hline
\end{tabular}

City, State, Zip

Mail To (if other than above): PGHts 9531 Band Rd.

City, State, Zip

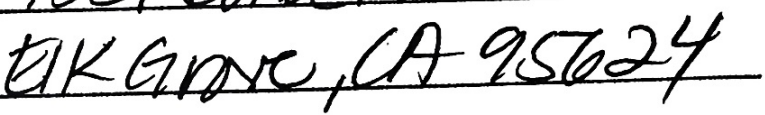

Reference/Explanation: Supplies fion Eakth Science Classactivityj supplies/food for Greenhand FFA Day.

ACCOUNT CODE

\begin{tabular}{|c|c|c|c|c|c|c|c|c|}
\hline \begin{tabular}{|l|} 
FUND \\
\end{tabular} & SITE & MGMT & FUNC & GOAL & RESC & $Y R$ & OBJECT & AMgyNTel \\
\hline 01 & 480 & 4250 & 1000 & 1700 & 7010 & 0 & 4310 & $\$ 305.26$ \\
\hline 81 & 480 & 2200 & 1000 & 1705 & 9000 & 0 & 4310 & S1 $\frac{378.846}{148.26}$ \\
\hline 01 & $40 \%$ & 43570 & 1078 & 1708 & 7010 & 0 & 4315 & $160^{-}$ \\
\hline a & 480 & 2200 & 1000 & 1705 & over) & 0 & 4315 & 160 \\
\hline
\end{tabular}

Component:

Activity \#

Page \#

Requested by:

Site Administrator Approval:

Site Controller Approval:

(Secondary Sites only)

Program Manager Approval:

Purchasing Director Approval:

Budget Approval:

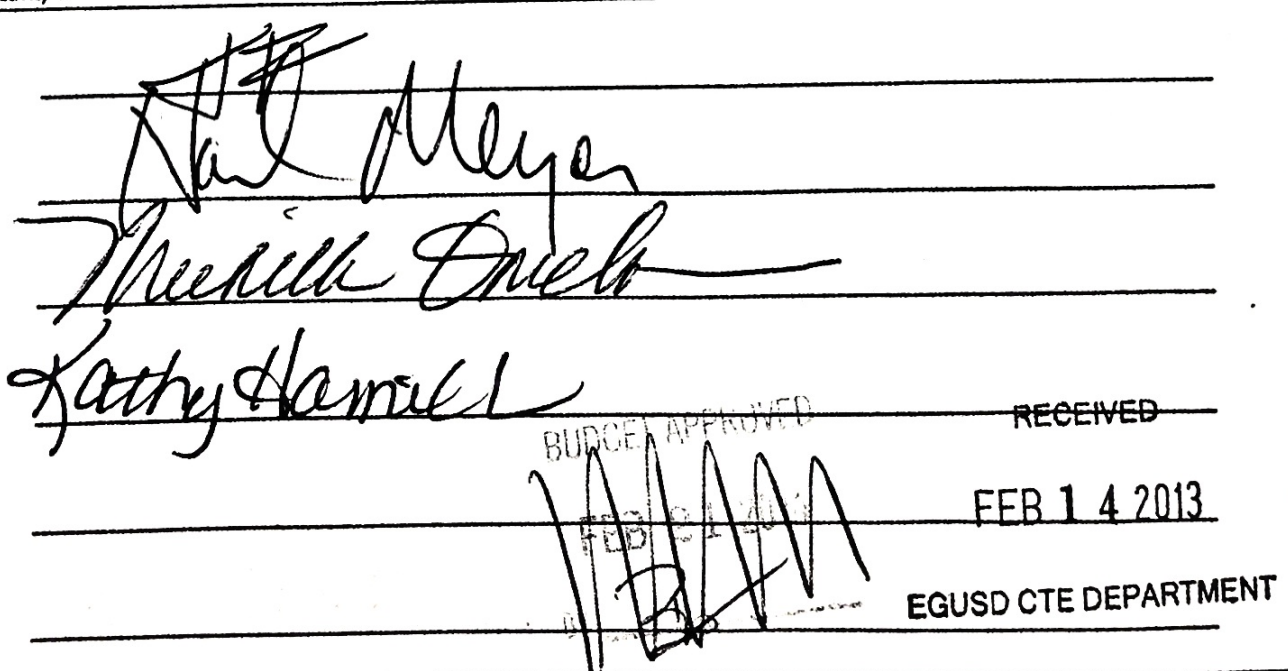




\section{Support Material 24: CATA Membership Card}




\section{Pleasant Grove High School Agriculture Department}

\section{Support Material 24: CATA Membership Card}

My CATA membership is paid through 2014, and is recorded on the Central Region CATA Membership Roster. Additionally, this year I am serving as the Sacramento Section CATA Vice President. 


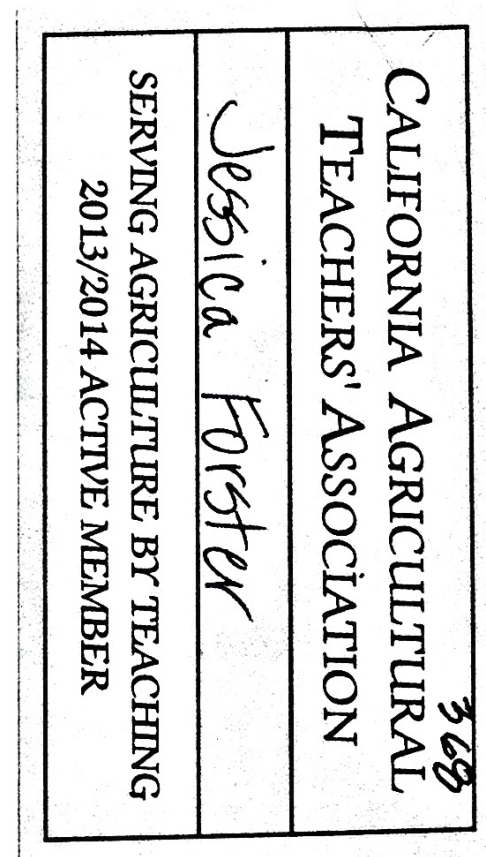


Support Material 25:

Report to

Administration 
Support Material 25:

\author{
Report to \\ Administration
}




\section{Pleasant Grove High School Agriculture Department}

\section{Support Material 25: Report to Administration}

Attached is a copy of a thank you note sent to our administrators for attending our Sectional CATA Administrators Night event, held each year in December. Our Administrators were all extremely impressed with the program at this year's event, and are looking forward to the annual event next year. 
December 17, 2014

Dear Mr. Meyer,

On behalf of the Pleasant Grove High School Agriculture Department, I would like to extend our sincere gratitude to you for attending our California Agriculture Teachers Association's Sectional Administrators Night. This annual event gives us time to share with all of the administrators within our Section the amazing things that our Agriculture Departments and students are accomplishing and to share some of our student's personal stories. We know that your time is extremely valuable, especially at this busy time of year, and are so appreciative of the fact that you chose to take time out of your day to attend this event. Your continued support of the Pleasant Grove High School Agriculture Department allows our students to continue developing their potential for premier leadership, personal growth, and career success trhough our agriculture and FFA Actvities.

Thank you again for your continued support of the Pleasant Grove High School Agriculture Department as well as our Sectional Administrator's Night.

Thank you,

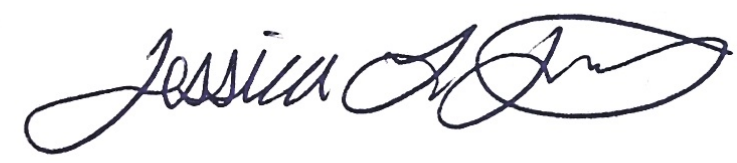

Jessica Forster,

Agriculture Instruct, FFA Advisor

Pleasant Grove High School 


\section{Support Material 26:}

Five Year Acquisition List 


\section{Pleasant Grove High School Agriculture Department}

\section{Support Material 26: Five Year Acquisition List}

The Five Year Acquisition List is an integral aspect of the Comprehensive Program Plan, and lists all major purchases and/or project goals that our agriculture department would like to accomplish over the next five years. This acquisition list is updated each year in accordance with the Agriculture Incentive Grant guidelines. 
Pleasant Grove High School Agriculture Department

5 Year Acquisition Schedule

Year $1 \quad$ 2012-2013

Quantity Description

1 Color Printer

10 Portable Digital Balances

Vendor

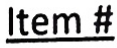

Unit Price

8 Graduated Cylinder, $10 \mathrm{~mL}$

Sargent-Welch

WLS1759-62

$\$ 150.00$

Sargent-Welch

WL5260B

$\$ 342.00$

8 Graduated Cylinder, $25 \mathrm{~mL}$

Sargent-Welch

WL5260C

$\begin{array}{ll}\$ & 3.65 \\ \$ & 3.95 \\ \$ & 4.95 \\ \$ & 6.70\end{array}$

Sargent-Welch

WL5260E

$\begin{array}{ll}\$ & 3.65 \\ \$ & 3.95 \\ \$ & 4.95 \\ \$ & 6.70\end{array}$

8 Graduated Cylinder, $100 \mathrm{~mL}$

Nasco

C11387N

$\begin{array}{ll}\$ & 3.65 \\ \$ & 3.95 \\ \$ & 4.95 \\ \$ & 6.70\end{array}$

10 Heat Lamps

Nasco

1 Popper Livestock Hauler

15 Introductory Horticulture, 10th Ed. Delmar/Amazon

32 Sunset Western Garden Book

Amazon

ISBN-13: 978-1401889524

$\$ 2,000.00$

1 Document Scanner

\section{Year 2}

Quantity

2

8

10

32 Goggles Hooded Vent UV FogFree

2 Heavy Duty Service Utility Carts

1 Thermometers, pkg of 25

1 Laminator

Year 3

Beaker 5 piece set

Wash Bottles, $500 \mathrm{~mL}, 6 \mathrm{pk}$

Hot Plate $4 \times 4$ Ceramic $120 \mathrm{~V}$

Hot Hand Protector

2014-2015

Quantity

30 Apron Rubber Cloth HW 2×30in

1 Network Copier

Year $4 \quad$ 2015-2016

Quantity Description

12 HP Computers

30 Ipads
Vendor

Sargent-Welch

Vendor

$\underline{\text { Vendor }}$

\begin{tabular}{crr} 
Item \# & \multicolumn{2}{c}{ Unit Price } \\
WLS4675-ZZ & $\$$ & 31.95 \\
WLS9486-05C & $\$$ & 31.25 \\
WLS1765-41 & $\$$ & 261.00 \\
WLS1774-22 & $\$$ & 20.35 \\
WLS40380-03A & $\$$ & 7.30 \\
WLS19758-B & $\$$ & 250.00 \\
WLS80030-AT & $\$$ & 295.00 \\
& &
\end{tabular}

Item \#

WLS1752-37

Unit Price

$\$ 11.55$

$\$ 1,700.00$
Year 5

Quantity

1 Chevrolet Suburban or Van

1 Gooseneck Trailer

2016-2017

Description
Item \#

Unit Price

$\$ 45,000.00$

$\$ 16,000.00$ 


\section{Support Material 27: Operating Budget for Deparment}




\section{Pleasant Grove High School Agriculture Department}

\section{Support Material 27: Operating Budget for Department}

The Department Chair, Izuskan Zallo, manages the Agriculture Incentive Grant and Perkins funding for our Agriculture Department. As the FFA Advisor, I manage the ASB FFA account. Included is the Perkins Application for 2013-2014, the 2013-2014 Agriculture Incentive Grant application, and the 2013-2014 ASB FFA Budget, and the 2013-2014 Floral Design Budget, which I oversee.

District budgets are not distributed on a regular basis, though we do receive allocations for each laboratory section taught in our program. This money is used to cover classroom and laboratory supplies. 


\begin{tabular}{|llc|}
\hline School & PLEASANT GROVE HIGH SCHOOL \\
\hline Department & AGRICULTURE & \\
\hline Teacher & IZASKUN ZALLO & \\
\hline Phone \# & $916-686-0230 ~ X 4934$ & Dollar \\
\hline \multicolumn{2}{c|}{ Item(s) Requested } & Amount \\
\hline \multicolumn{2}{c|}{ Transportation (EGUSD Vehicles) } \\
2. Conference Registration (for advisors) & $\$ 2500.00$ \\
3. Livestock Shears/Show Equipment & $\$ 1750.00$ \\
\hline
\end{tabular}

\section{Please explain how the requested items will enhance student CTE learning}

\section{Rationale}

Part of the agriculture education program involves the development of skills and abilities in the areas of premier leadership, personal growth and career success. Students travel to various conferences and competitions throughout the state during the year. Advisors are required to attend certain events in order to comply with the Agriculture Incentive Grant. The department has a vehicle, but must rely on transportation provided by the EGUSD or personal vehicles to take students to/from events. The program has an enrollment of approximately 425 students and 3 teachers. Thus, the number of activities in which our program participates in has expanded along with the number of students involved. As a result our animal science projects have increased in numbers. We have a greater number of students interested in raising animals for the Sacramento County Fair and thus need equipment to meet their needs.

Program Impact - (How specifically will request affect student CTE learning)

Over $80 \%$ of the students in the program participate in FFA events at the local, state and national level. Last year, 25 students traveled to the State FFA Conference and over 40 students exhibited animals at the Sacramento County Fair. During the Spring, students participate in Career Development Events (CDES) at various field days. Currently, we have approximately 40 students who have an interest in a CDE.

Funding received by $\mathrm{Ag}$. Incentive Grant must be matched and our site funding is not enough to meet this. In order for the program to meet all requirements to be viable and in good standing with California FFA, extra funding is needed.

Without assistance through Carl D. Perkins, advisors will have to limit the participation in leadership activities during the year. Program would need to rely on parent volunteers for transportation of students. Lack of funding would jeopardize the funding received by $\mathrm{Ag}$. Incentive Grant.

What other available funds will be used to leverage this request?

Agriculture Incentive Grant; Site Funds; Aggie Backers (Ag Booster Club)

Department Chair Signature

School Site Principal Signature 
California Department of Education

AGRICULTURAL CAREER TECHNICAL EDUCATION INCENTIVE GRANT 2013-14 APPLICATION FOR FUNDING

(Due Date: To be received in Regional Supervisor's Office by June 30, 2013)

DATES OF PROJECT DURATION - JULY 1, 2013, TO JUNE 30, 2014

PLEASANT GROVE HIGH SCHOOL

(School Site)
ELK GROVE UNIFIED SCHOOL DISTRICT

(District)

Certification: I hereby certify that all applicable state and federal rules and regulations will be observed; that to the best of my knowledge, the information contained in this application is correct and complete; and that the attached assurances are accepted as the basic conditions of the operations in this project/program for local participation and assistance.

Signature of Authorized Agent

Signature of Agriculture Teacher

Responsible for the Program

Date of Approval of Local Agency Board:

Funds Requested - Part I

Part II

Part III

Part IV

Total
Title

Signature of Principal

Contact Phone Number:

916-686-0230

\section{$\$ 12,520.00$}

\begin{tabular}{c}
\hline$\$ 5,000.00$ \\
\hline$\$ 3,520.00$ \\
\hline$\$ 4,000.00$ \\
\hline$\$ 0.00$ \\
\hline
\end{tabular}

3

Number of Different Agriculture Teachers at Site:

PART I - QUALITY CRITERIA 1-9 (REQUIRED) ALLOCATION

Quality Criteria

$\begin{array}{cc}\text { Will Meet } & \text { Variance } \\ \text { Criteria } & \text { Requested }\end{array}$

1. Curriculum and Instruction

2. Leadership and Citizenship Development

3. Practical Application of Occupational Skills

4. Qualified and Competent Personnel

5. Facilities, Equipment, and Materials

6. Community, Business, and Industry Involvement

7. Career Guidance

8. Program Promotion

9. Program Accountability and Planning

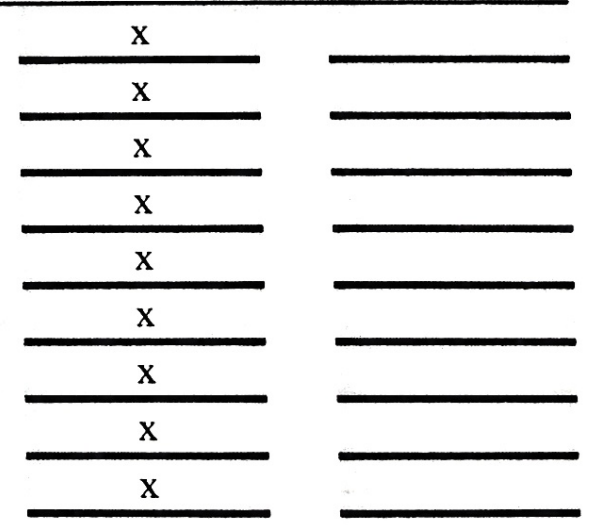

Formal Variance Request must be included if requesting a variance. A variance is a proposed plan for bringing the program into compliance with required quality criteria. Variances should result in compliance prior to the following year's application. All variances must be approved with the application. Non-compliance with the terms of the approved variance will result in a loss of funds. 


\section{PART I - CONTINUED}

Departmental Allocation: Meeting the criteria in PART I makes the program eligible for the following amounts based on the number of teachers in the program.

\begin{tabular}{lcc} 
Total Number of Teachers & Amount Eligible & Amount Requested \\
\hline One Teacher or Less & $\$ 4,000$ & \\
Two Teachers & $\$ 4,500$ & \\
Three Teachers or More & $\$ 5,000$ & $\$ 5,000.00$ \\
\hline
\end{tabular}

PART II - PROGRAM ENROLLMENT ALLOCATION

Total Number of Students 2012-13 R2 Number Amount Requested

List Number from R2 Report (\$8/Member) . $\quad 440 \quad \$ 3,520.00$

\section{PART III - QUALITY CRITERIA 10-11 (OPTIONAL) ALLOCATION}

Schools which qualify for a Departmental Allocation may apply for additional amounts for each specific Quality Criteria (10 and 11) met.

* Amounts requested in Quality Criterion 10 will be the indicated amount for that criterion, multiplied by the full-time equivalent (FTE). To count a preparation period, the teacher must be teaching Career Technical Education courses in Agriculture for 50 percent or more of their teaching periods.

* Amounts requested in Quality Criterion $11 A$ will be the indicated amount for each teacher who was compensated a minimum of $\$ 2,000$ for year-round employment.

* Amounts requested in Quality Criterion $11 \mathrm{~B}$ will be the indicated amount for each teacher who is provided a project supervision period. Project periods will be counted if the teacher has a preparation period as part of the regular teaching day.

Number of FTE Agriculture Teachers at Site:

3

List the Names of the Agriculture Teachers:

1. Izaskun Zallo

2. Jessica Forster

3. To Be Announced

Criterion 10 - Student/Teacher Ratio

Criterion 11A - Year-Round Employment

Criterion 11B - Project Supervision Period

TOTAL FUNDS REQUESTED PART IV
4.

5.

6.

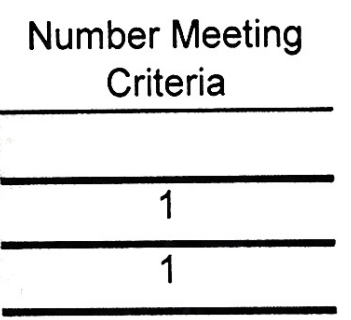

Amount

Requested

$\$ 0.00$

$\$ 2,000.00$

$\$ 2,000.00$

$\$ 4,000.00$

\section{PART IV - QUALITY CRITERION 12 (OPTIONAL) ALLOCATION}

Quality Criterion 12 Form is attached and all criteria has been met. If the answer is yes, list $\$ 7,500$ (funds requesting) in space to the right. 
PART V - FINANCIAL SCHEDULE

Part A

\begin{tabular}{|c|c|c|c|c|c|c|}
\hline & & & $\bar{A}$ & $B$ & & C \\
\hline & $\begin{array}{l}\text { Acct. } \\
\text { No. }\end{array}$ & Classification & $\begin{array}{c}\text { Description of Item for } \\
\text { Which Funds Will be } \\
\text { Expended }\end{array}$ & $\begin{array}{c}\text { Incentive } \\
\text { Grant Funds }\end{array}$ & & $\begin{array}{l}\text { Matching } \\
\text { Funds }\end{array}$ \\
\hline 1 & 4000 & Books \& Supplies & 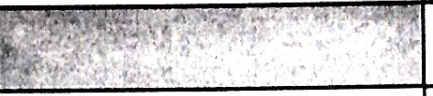 & $4,668.00$ & & $4,668.00$ \\
\hline 2 & 2.:- & (x) & Subtotal for 4000 & $\$ 4,668.00$ & & $\$ 4,668.00$ \\
\hline 3 & \multirow[t]{6}{*}{5000} & \multirow{6}{*}{$\begin{array}{l}\text { Services and Other Operating } \\
\text { Expenses such as: Services of } \\
\text { Consultants, Staff Travel, and } \\
\text { Conference; Rentals, Leases, and } \\
\text { Repairs; Bus Transportation }\end{array}$} & 1. Travel/Conference & $2,500.00$ & & $2,500.00$ \\
\hline 4 & & & 2. Maint. Agreements & 800.00 & & 800.00 \\
\hline 5 & & & 3. Field Trips (Transp.) & $3,000.00$ & & $3,000.00$ \\
\hline 6 & & & 4. Vehicle Replace Fee & 986.00 & & 986.00 \\
\hline & & & 5. Vehicle Repair & 566.00 & & 566.00 \\
\hline 7 & & & 6. & & 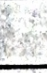 & \\
\hline 8 & 80 & (3) & Subtotal for 5000 & $\$ 7,852.00$ & & $\$ 7,852.00$ \\
\hline 9 & \multirow[t]{5}{*}{6000} & \multirow{5}{*}{$\begin{array}{l}\text { Capital Outlay: Includes Sites and } \\
\text { Improvements of Sites; Buildings and } \\
\text { Improvement of Buildings; Equipment }\end{array}$} & 1. & & 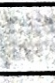 & \\
\hline 10 & & & 2. & & 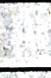 & \\
\hline 11 & & & 3. & & 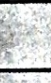 & \\
\hline- & & & 4. & & 8 & \\
\hline 72 & & & 5. & & & \\
\hline 13 & $2=$ & 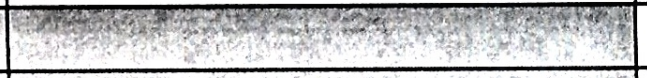 & Subtotal for 6000 & $\$ 0.00$ & & $\$ 0.00$ \\
\hline 14 & 3 & 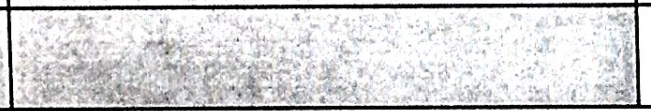 & $\begin{array}{c}\text { Total for } 4000-6000 \\
\text { Lines } 2,8,13\end{array}$ & $\$ 12,520.00$ & & $\$ 12,520.00$ \\
\hline
\end{tabular}

TOTAL 2013-14 Incentive Grant Allocation:

$\$ 12,520.00$

Part B - Complete this portion if a waiver of the matching requirement is requested:

\begin{tabular}{|c|c|c|c|c|c|c|}
\hline & & & $\mathrm{A}$ & $B$ & & $C$ \\
\hline Line & $\begin{array}{l}\text { Acct } \\
\text { No. }\end{array}$ & Classification & $\begin{array}{c}\text { Description of Item for } \\
\text { Which Funds Were } \\
\text { Expended }\end{array}$ & $\begin{array}{c}\text { Incentive } \\
\text { Grant Funds }\end{array}$ & & $\begin{array}{c}\text { Amount of } \\
\text { Salary and } \\
\text { Benefits } \\
\end{array}$ \\
\hline 15 & 1000 & Salaries & $\begin{array}{l}\text { Teachers' Summer } \\
\text { Service Salaries }\end{array}$ & 8 & 3 & \\
\hline 16 & 1000 & Salaries & $\begin{array}{l}\text { Teachers' Salaries for } \\
\text { Project Supervision } \\
\text { Period }\end{array}$ & & 4 & \\
\hline 17 & 3000 & Benefits & $\begin{array}{l}\text { Benefits for the Above } \\
\text { Items }(1000)\end{array}$ & 2 & 2. & \\
\hline 18 & & 30 & TOTAL & 5 & 4 & $\$ 0.00$ \\
\hline
\end{tabular}

TOTAL Amount of Waiver Requested: 
Please complete ALL areas of this form.

\section{Pleasant Grove High School \\ Associated Student Body}

CLUBIACCOUNT BUDGET FORM

\section{Club/Account Name: PG FFA}

\begin{tabular}{l}
$\begin{array}{l}\text { Budget for } \\
\text { School Year: }\end{array}$ \\
Today's Date: $\quad 2013-2014$ \\
$\begin{array}{l}\text { Club/Account } \\
\text { Number: }\end{array}$ \\
\hline
\end{tabular}

Number:

Each Club/Account operating through the ASB Account must complete a Budget Form for the current school year. Please complete this form and submit it to the Controller within $\mathbf{3 0}$ days from the start of school. If you need help on preparing the form please refer to the instructions in your copy of the Associated Student Body Forms \& Information Guide on page 2a.

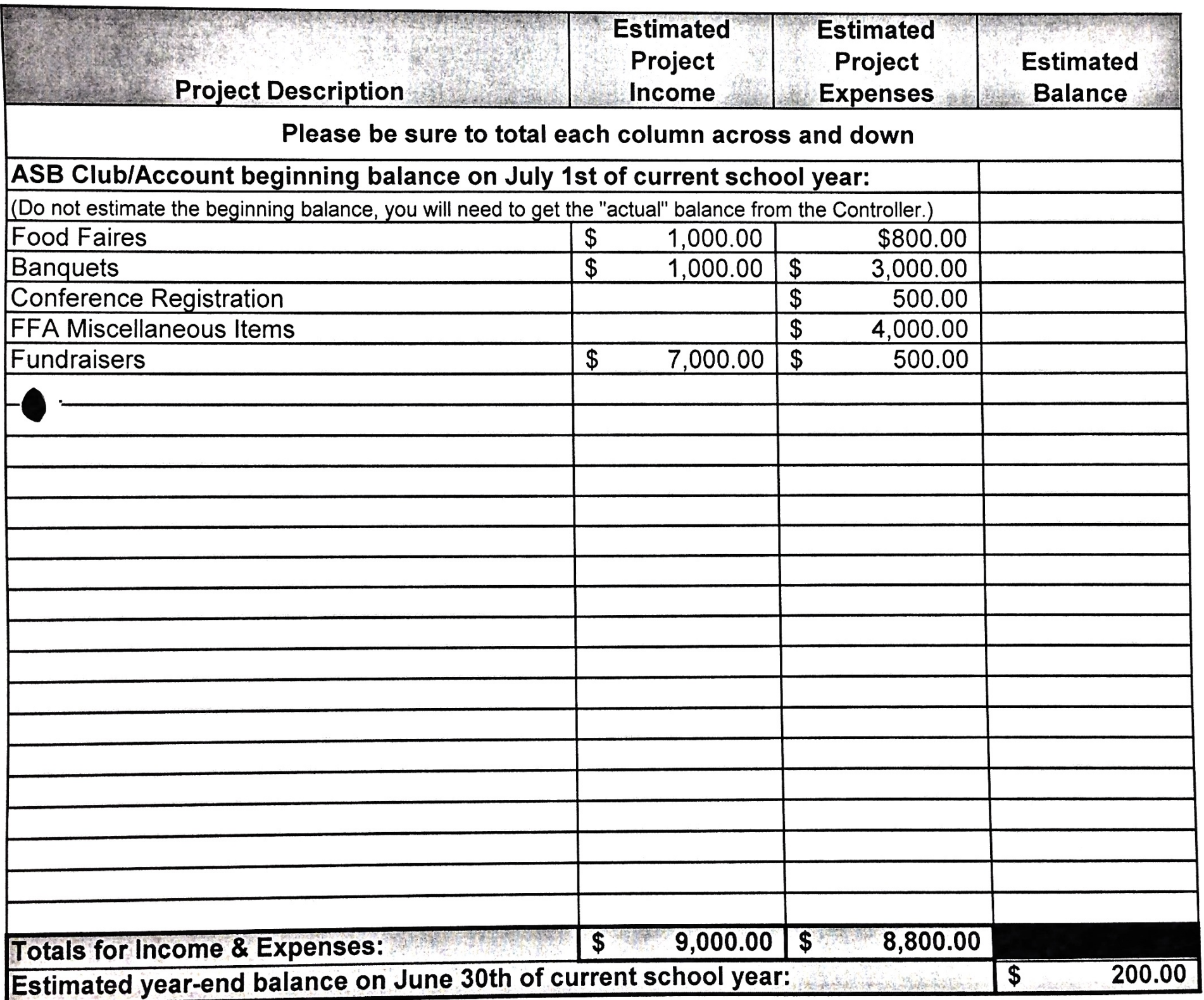

S, ature of Club/Account Advisor:

Date of minutes when this budget was approved by Club/Account:

(Please submit along with this completed form a copy of your minutes showing your budget approval, the minutes must be signed by the club advisor and a student club representative.) 
Please complete ALL areas of this form.

\section{Pleasant Grove High School \\ Associated Student Body \\ CLUBIACCOUNT BUDGET FORM}

\begin{tabular}{ll|}
\hline Club Name: & Ag - Floral \\
School Year: & $2013-2014$ \\
Today's Date: & $8 / 16 / 2013$ \\
\hline
\end{tabular}

Club/Account

Number:

$300-0500$

\section{Club/Account Name: Ag - Floral}

Each Club/Account operating through the ASB Account must complete a Budget Form for the current school year. Please complete this form and submit it to the Controller within 30 days from the start of school. If you need help on preparing the form please refer to the instructions in your copy of the Associated Student Body Forms \& Information Guide on page 2a.

\begin{tabular}{|c|c|c|c|}
\hline Project Description & $\begin{array}{c}\text { Estimated } \\
\text { Project } \\
\text { Income }\end{array}$ & $\begin{array}{c}\text { Estimated } \\
\text { Project } \\
\text { Expenses }\end{array}$ & $\begin{array}{c}\text { Estimated } \\
\text { Balance }\end{array}$ \\
\hline
\end{tabular}

Please be sure to total each column across and down

ASB Club/Account beginning balance on July 1st of current school year:

(Do not estimate the beginning balance, you will need to get the "actual" balance from the Controller.)

Flowers (Sales \& Expenditures)

Donation

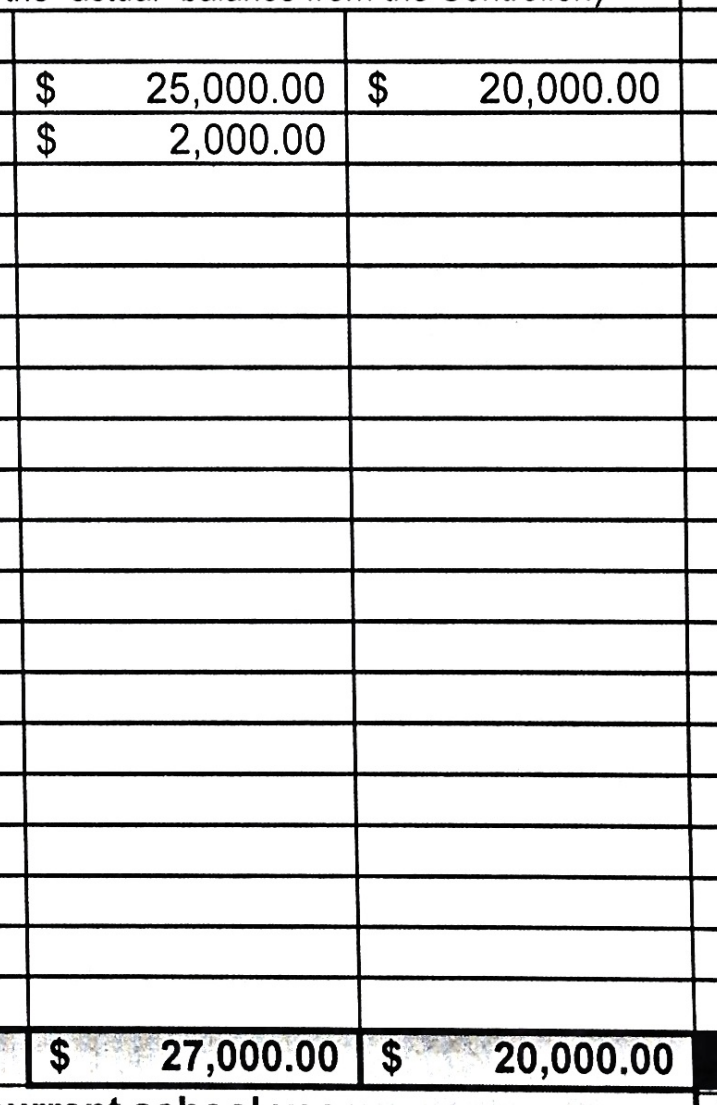

Totals for Income \& Expenses:

\begin{tabular}{|lr|rr|}
\hline$\$ 27,000.00$ & $\$ 20,000.00$ & & \\
\hline
\end{tabular}

Signature of Club/Account Advisor:

Signature of Club/Account Student Representative:

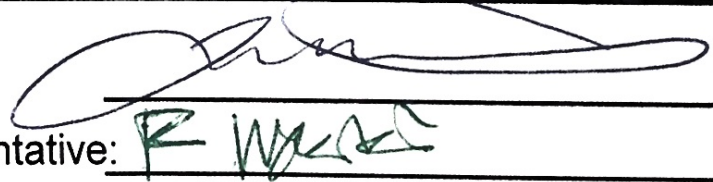

S: ature Principal:

Date of minutes when this budget was approved by Club/Account:

(Please submit along with this completed form a copy of your minutes showing your budget approval, the minutes must be signed by the club advisor and a student club representative.) 
- $\quad$ Support Material 28: Budget Process 


\section{Pleasant Grove High School Agriculture Department}

\section{Support Material 28: Budget Process}

The budget process in described within the Elk Grove Unified School District's Purchasing Department Guide, which is attached. Regardless of how an expenditure will be funded, all purchases must be pre-approved through the individual department, school Site Controller and Administration, and the district Purchasing Department. All ASB reimbursements must be either pre-approved, or run through an open Purchasing Order. 


\section{ELK GROVE UNIFIED SCHOOL DISTRICT \\ PURCHASING DEPARTMENT \\ INFORMATION AND PROCEDURE GUIDE}

The ELK GROVE UNIFIED SCHOOL DISTRICT services students from kindergarten through twelfth grade with an enrollment of approximately 61,500 students plus and Adult Education Program.

In accordance with the District's centralized purchasing policy, the Purchasing Department has the general responsibility for the purchase of all materials, supplies and equipment.

Competitive bidding, verbal and written quotations are used as a means to procure needed products and services at the best price for the quality which best meets the District's requirements.

The Facilities Department seeks quotations and formal bids for public works contracts such as asphalt, roofing, asbestos removal, etc.. Facilities has the responsibility of bidding for construction of buildings and acquiring additional properties.

\section{VENDORS:}

Vendors may be added to our bidders' and resource lists by sending a letter on company letterhead to the Purchasing Department via mail or fax. The letter should include: business license number, listing of products and/or Services offered, and contact person.

Vendors wishing to meet with a Buyer should call for an appointment.

\section{AUTHORIZED PURCHASES:}

A purchase order is issued for all purchases. Only the Purchasing staff has the authority to issue or revise purchase orders. Purchase order numbers may be given by telephone, when necessary to expedite delivery. But only by the Purchasing Staff.

The Purchasing Department staff wishes to cultivate and maintain professional relationships with all vendors and continue to increase our knowledge of products and equipment available. Therefore, vendors are required to conduct their business directly with the Purchasing Office. Vendors many not visit District sites with out prior authorization.

When appropriate, the Buyer will make arrangements for demonstrations whether at school locations or at the vendors business location. Buyers will attend most demonstrations.

Purchases made by the Facilities/Maintenance Department for emergency repair parts or for materials necessary for continuing work in progress also requires purchase order numbers. Purchase Order numbers are issued by Purchasing to the Facilities/Maintenance Department staff to obtain these types of materials.

Orders accepted by the vendor without the issuance of a purchase order by Purchasing or Facilities/Maintenance will be considered the responsibility of the individual placing the order and will not be considered an obligation of the District. 
It is therefore, important to contact the Purchasing Department regarding any orders placed by other District personnel prior to acceptance or the order.

All Contracts must be reviewed and signed by authorized district office personnel prior to enactment. Any contracts signed by a site representative is not enforceable prior to review. Please contact the Purchasing Department for additional information on contracts.

\section{FORMAL BIDS}

Formal bids are required for supplies and equipment excess of $\$ 84,100$ and labor in excess of $\$ 15,000.00$. These amounts are in accordance with the PCC20111 and may increase annually.

Legal advertisements for formal bids are printed in a local newspaper, such as the Elk Grove Citizen, that is of general circulation stating the title of the bid and the date of opening. Formal bids are opened at the time advertised for opening. Bids received after the opening time will not be accepted if mailed, late bids will be returned to the bidder unopened.

It is not required, but bidders may attend the public openings of formal bids. Upon opening, bids are read aloud. No decisions for award are made at the time of opening. All bids must be ratified by the EGUSD Board of Education

Award is made to the lowest responsible bidder(s) meeting or exceeding the specifications for the service or product to be purchased and in accordance with all the terms and conditions of the bid.

\section{DELIVERIES:}

All deliveries shall be made to the site designated on the purchase order in the "ship to" space. Deliveries made to sites other than those indicated on the purchase order will delay the clearing of the purchase order and will cause a delay in payment. The purchase order number must be indicated on all packages and packing slips.

\section{INVOICING AND PAYMENT:}

Itemized invoices indicating the purchase order number are to be mailed in duplicate to the attention of Accounts Payable at 9510 Elk Grove-Florin Road, Elk Grove, CA 95624.

Payment may be expected within thirty (30) days of delivery and receipt of correct merchandise and an invoice. Terms of less than 30 days are not considered when determining the award of bids and quotes.

MATERIAL SAFETY DATA SHEETS (MSDS):

Vendors are required to provide Material Safety Data Sheets from the manufacturer for all products that are hazardous, as defined by Title 8 of the California Code of Regulations. Failure to do so may be cause for delay of payment. 


\section{Support Material 29:}

\section{Chair Person's Duties}

\section{- \& Responsibilities}


Pleasant Grove High School Agriculture Department

Support Material 29: Chair Person's Duties \& Responsibilities

I do not currently serve as the Agriculture Department Chair at this time. 


\section{Support Material 30:}

\section{Chart of}

\section{Responsibilities}




\section{Pleasant Grove High School Agriculture Department}

\section{Support Material 30: Chart of Responsibilities}

The Chart of Responsibilities is extremely important to ensuring the variety of activities and duties within our department are accomplished in a timely manner. This chart makes it explicitly clear who is responsible for each activity, and is updated each year as a part of the Comprehensive Program Plan. 


\section{Pleasant Grove High School Ag Department}

Staff Assignments 2013-2014

Zallo Forster Swenson

Ag Department

\begin{tabular}{|l|l|l|l|}
\hline Department Chair & $\mathrm{X}$ & & \\
\hline FFA Advisor & & $\mathrm{X}$ & \\
\hline
\end{tabular}

Animal/Livestock

Fair Supplies (per species)

Veterinary Supplies (per species)

\begin{tabular}{|c|c|c|}
\hline $\mathrm{X}$ & $\mathrm{X}$ & $\mathrm{X}$ \\
\hline $\mathrm{X}$ & $\mathrm{X}$ & $\mathrm{X}$ \\
\hline
\end{tabular}

Accounting

Dept/District Acct/PO's

FFA Acct / PO's

Perkins Funding Application

\begin{tabular}{|c|c|c|c|}
\hline$X$ & & \\
\hline & & $X$ & \\
\hline & $X$ & & \\
\hline
\end{tabular}

General Program/Facility

$\mathrm{Ag}$ Advisory Comm Minutes

Ag Advisory Comm Planning \& Agenda

Department Marketing / PR

Maintenance Requests

Transportation Requests

Greenhouse

Large Animal Barn (by species)

Small Animal Barn (by species)

Pleasant Grove HS Aggie Backers Liaison

AG Truck Maintenance/Repairs

\begin{tabular}{|c|c|c|}
\hline & & $X$ \\
\hline$X$ & $X$ & $X$ \\
\hline$X$ & & \\
\hline$X$ & & \\
\hline$X$ & $X$ & \\
\hline$X$ & $X$ & $X$ \\
\hline$X$ & & \\
\hline$X$ & & \\
\hline
\end{tabular}

Equipment/Tools

Livestock Trailer

Fair Equipment \& Tools (per species)

Storage Building

\begin{tabular}{|c|c|c|}
\hline $\mathrm{X}$ & & \\
\hline $\mathrm{X}$ & $\mathrm{X}$ & $\mathrm{X}$ \\
\hline $\mathrm{X}$ & & \\
\hline
\end{tabular}

Annual Reports

5-Year Equipment Allocation

Advisory Committee Roster \& Minutes

American FFA Degree Applications

Chart of Staff Responsibilities

Comprehensive Program Plan

FFA Activities Calendar

FFA Award Applications

FFA Membership Roster \& Dues (Each teacher enters own)

FFA Program of Activities

Graduate Follow-Up

Incentive Grant

Incentive Grant Reviews

Recruitment

Report of Expenditures

State FFA Degree Applications

\begin{tabular}{|c|c|c|}
\hline$X$ & & \\
\hline$X$ & $X$ & $X$ \\
\hline$X$ & & \\
\hline$X$ & & \\
\hline$X$ & $X$ & \\
\hline$X$ & $X$ & $X$ \\
\hline & $X$ & \\
\hline$X$ & & \\
\hline$X$ & & \\
\hline & $X$ & \\
\hline$X$ & & \\
\hline & $X$ & $X$ \\
\hline
\end{tabular}


Project Supervision

\begin{tabular}{|c|c|c|c|}
\hline Beef Cattle & & $\mathrm{X}$ & \\
\hline Dairy Cattle & & $\mathrm{X}$ & \\
\hline Goats & $\mathrm{X}$ & & \\
\hline Horses & & & $\mathrm{X}$ \\
\hline Floriculture & & $\mathrm{X}$ & \\
\hline Horticulture & & $\mathrm{X}$ & \\
\hline Poultry & & & $\mathrm{X}$ \\
\hline Rabbit & & $\mathrm{X}$ & \\
\hline Sheep & $\mathrm{X}$ & & \\
\hline Swine & & & $\mathrm{X}$ \\
\hline Work Experience & $\mathrm{X}$ & $\mathrm{X}$ & $\mathrm{X}$ \\
\hline
\end{tabular}

FFA/CATA

Activities

\begin{tabular}{|c|c|c|c|}
\hline FFA Officer Retreat & & $\overline{\bar{X}}$ & \\
\hline Quarterly/Yearly CATA Meetings/Events & $\mathrm{X}$ & & \\
\hline Jr. Livestock Auction Committee (county fair) & & $\mathrm{X}$ & \\
\hline Greenhand Conference & & & $\mathrm{X}$ \\
\hline Chapter Officer Leadership Conference & & $\mathrm{X}$ & \\
\hline Opening/Closing Ceremonies Contest & & $\mathrm{X}$ & $\mathrm{X}$ \\
\hline National FFA Convention & & $\mathrm{X}$ & \\
\hline Administrator/Counselor Night & & $\mathrm{X}$ & \\
\hline Organize Local \& Sectional Project Competition & & $\mathrm{X}$ & \\
\hline Organize Local Speaking Contests & & $\mathrm{X}$ & \\
\hline Sectional Speaking Contests & & $\mathrm{X}$ & \\
\hline MFE/ALA Conferences & & $\mathrm{X}$ & \\
\hline Spring Regional Meeting & $\mathrm{X}$ & & \\
\hline UC Davis Field Day & $\mathrm{X}$ & & \\
\hline CSU Chico Field Day & $\mathrm{X}$ & & \\
\hline Modesto JC Field Day & $\mathrm{X}$ & & \\
\hline Cosumnes River College Field Day & $\mathrm{X}$ & & \\
\hline CSU Fresno Field Day & $\mathrm{X}$ & & \\
\hline Ag Day at the Capitol & $\mathrm{X}$ & $\mathrm{X}$ & $\mathrm{X}$ \\
\hline State FFA Leadership Conference & & & $\mathrm{X}$ \\
\hline Cal Poly, SLO State FFA Finals & $\mathrm{X}$ & $\mathrm{X}$ & \\
\hline Sectional FFA Meeting & $\mathrm{X}$ & & \\
\hline Sacramento County Fair & $\mathrm{X}$ & $\mathrm{X}$ & $\mathrm{X}$ \\
\hline California State Fair & & $\mathrm{X}$ & \\
\hline
\end{tabular}

Fundraisers

\begin{tabular}{|l|c|c|c|}
\hline Donkey Basketball & & $\mathrm{X}$ & \\
\hline Football Fridays & $\mathrm{X}$ & & \\
\hline & & & \\
\hline & & & \\
\hline & & & \\
\hline & & & \\
\hline
\end{tabular}


FFA Judging Teams/Contests

Zallo Forster Swenson

\begin{tabular}{|l|c|c|c|}
\hline AG Issues & & & \\
\hline AG Sales & & & \\
\hline BIG & & & $\mathrm{X}$ \\
\hline COOP Quiz & & $\mathrm{X}$ & \\
\hline Creed Speaking & & & \\
\hline Dairy Products & & $\mathrm{X}$ & \\
\hline Extemporaneous Speaking & & & \\
\hline Farm Business Management & & $\mathrm{X}$ & \\
\hline Farm Records & & $\mathrm{X}$ & \\
\hline Job Interview & & & \\
\hline Livestock & & & \\
\hline Marketing Plan & & & \\
\hline Opening/Closing Advanced & & & \\
\hline Opening/Closing Novice & & & \\
\hline Parliamentary Procedure & & & $\mathrm{X}$ \\
\hline Poultry & $\mathrm{X}$ & & \\
\hline Prepared Public Speaking & & & \\
\hline Specialty Animals & $\mathrm{X}$ & & \\
\hline
\end{tabular}

Awards

\begin{tabular}{l|c|c|c|}
\hline Greenhand Degrees & & & $\mathrm{X}$ \\
\hline Chapter Degrees & $\mathrm{X}$ & & \\
\hline Awards Banquet & & $\mathrm{X}$ & \\
\hline National Chapter Awards & & $\mathrm{X}$ & \\
\hline Scholarships & $\mathrm{X}$ & $\mathrm{X}$ & \\
\hline Top 10 Tabulations (w/ Reporter) & & $\mathrm{X}$ & \\
\hline Proficiency Awards & $\mathrm{X}$ & $\mathrm{X}$ & $\mathrm{X}$ \\
\hline
\end{tabular}




\section{Support Material 31:}

Substitue Teacher

Procedure \& Plans 


\section{Pleasant Grove High School Agriculture Department}

\section{Support Material 31: Substitute Teacher Procedure \& Plans}

When it is required for me to be off campus for a professional development activity or student event, all certified employees must complete a Absence Request Form and sent to our Principal's Secretary to have the request approved and a substitute arranged.

I maintain a Substitute Binder, which includes my lesson plans, instructions for working our integrated LCD projector system, and seating charts for each class period. Unfortunately, substitutes in our district do not always have a the credentials/password necessary to access our district computers, so normally my substitute plans include sutdent worksheets, group activities that continue with a lesson taught the previous day, or an educational film and worksheet.

I have included a copy of our school's Absence Request Form, as well as a copy of a recent set of substitue plans. 


\section{CERTIFICATED ABSENCE NOTIFICATION (Site Use Only)}

Administrative Signature:

Date:

(Site Administration must sign off on this absence form before you request a substitute.)

Name:

Social Security No.

Absence Reason:

Date of Absence:

Time:

Pre-Arranged Substitute:

Yes No Name:

Who is paying for the Substitute?

Are students involved? ___ Yes _ _ No If yes, has transportation been arranged? __ Yes _ _ No

*IMPORTANT NOTICE: Requests must be called into SEMS (Substitute Employee Management System) 10 days prior to absence.

Call 686-1250 to report this absence and obtain a job number. JOB NUMBER

This completed form should be given to your Secretary after you have obtained the job number. DO NOT SEND THIS FORM TO HUMAN RESOURCES

\section{CERTIFICATED ABSENCE NOTIFICATION (Site Use Only)}

Administrative Signature:

Date:

(Site Administration must sign off on this absence form before you request a substitute.)

Name:

Social Security No.

Absence Reason:

Date of Absence:

Time:

Pre-Arranged Substitute: Yes No Name:

Who is paying for the Substitute?

Are students involved? __ Yes _ _ No If yes, has transportation been arranged? __ Yes __ No

*IMPORTANT NOTICE: Requests must be called into SEMS (Substitute Employee Management System) 10 days prior to absence.

all 686-1250 to report this absence and obtain a job number. JOB NUMBER

This completed form should be given to your Secretary after you have obtained the job number. DO NOT SEND THIS FORM TO HUMAN RESOURCES 


\section{Good Morning!}

Sub Plans for Ms. Jessica Forster, Tuesday, April $22^{\text {nd }}$

Thank you for subbing for me today! My seating charts can be found in the front of my attendance binder in the sheet protectors - please feel free to use it to assist with taking role. My students know that they are to be in their assigned seats and ready to work within one minute of the bell ringing - if they aren't or if you have any type of problem with a student please leave a note and I will deal with them with I return.

Please do not allow students to move about the room - they should stay in their assigned seats only - no students are to be at lab stations unless it is their assigned seats. Please do not let them wander about the room. In the event that a student needs to use the restroom, they must present you with a bathroom pass (Floral design classes don't have bathroom passes) and use the keys in the top drawer of the front lab desk.

If you have any questions, please feel free to contact me at (916) 747-0711.

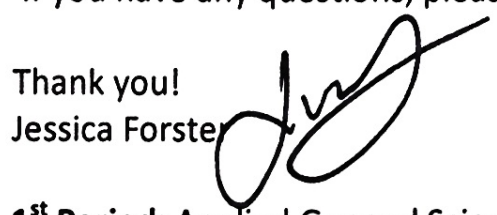

$1^{\text {st }}$ Period: Applied General Science:

Students in these classes know they must write down the daily objective and gee whiz (provided below for you to write on the board) at the beginning of the class period - they should do this within the first two minutes or so of class.

Objective: Today I begin reviewing correct animal nomenclature.

Gee Whiz: A group of flamingos is called a flamboyance.

Students in this class have two assignments today. The first is a Word Search for their animal science unit, which is already included in their packet. Students will need to have this stamped by the end of the class period, which should only happen after you have verified that they have all of the words circled/highlighted/found. The second assignment is to begin a review quiz for animal nomenclature. For this assignments, students must write their own quiz regarding animal nomenclature - it must be a least 25 questions and can be written as true/false, multiple choice or matching questions. Students must write a key for their quiz on a separate sheet of paper, which should be stapled to their quiz. Both the quiz and the key are due at the end of the class period.

$2^{\text {nd }}, 3^{\text {rd }}, 4^{\text {th }}$, and $6^{\text {th }}$ Period: Floral Design

Students will be working on a set of questions from their floral textbooks (class set is in the classroom). These questions need to be completed in complete sentences on page 47 of their Floral Notebook. Please stamp the assignment when students finish.

$7^{\text {th }}$ Period: Prep - enjoy!

T.A.'s: I have a number of T.A.'s throughout the day, which I have listed here by period: $1^{\text {st }}$ Period: Matthew Nielson, $2^{\text {nd }}$ Period: Mackenzie Telford, $3^{\text {rd }}$ Period: Sarah Fisher, $4^{\text {th }}$ Period: Kaitlyn Tobin, $5^{\text {th }}$ Period: Nicole Gillin \& Barrett Green, $6^{\text {th }}$ Period: Kelsie Kennicutt, $7^{\text {th }}$ Period: David Kammerer $\&$ Kelsie Kennicutt

TAs throughout the day can grade the Spring Final Exams from first period - a key is provided with these sub plans. 
6

Support Material 32:

- Description of Program Completer 


\section{Pleasant Grove High School Agriculture Department}

\section{Support Material 32: Description of Program Completer}

The description of what constitutes as Program Completer in the Agriculture Department at Pleasant Grove High School is a part of our Comprehensive Program Plan. To be considered a Program Completer and earn the right to wear a blue Agriculture Department cord at graduation, students must be enrolled in at least three years of Agriculture Education, including their senior year and must complete a designated pathway.

Our definition of a Program Completer was recently changed at our winter Advisory Committee Meeting, so I have attached the minutes from that meeting, and highlighted the portion pertaining to our discussion of Program Completers 


\section{Pleasant Grove High School Agriculture Department}

\section{Support Material 32: Description of Program Completer}

The description of what constitutes as Program Completer in the Agriculture Department at Pleasant Grove High School is a part of our Comprehensive Program Plan. To be considered a Program Completer and earn the right to wear a blue Agriculture Department cord at graduation, students must be enrolled in at least three years of Agriculture Education, including their senior year and must complete a designated pathway.

Our definition of a Program Completer was recently changed at our winter Advisory Committee Meeting, so I have attached the minutes from that meeting, and highlighted the portion pertaining to our discussion of Program Completers 
In attendance: Michelle Doiron, Dan Forster, Jessica Forster, Lynn Martindale, Angela Mayfield, Jenna Swenson, Izzy Zallo

The meeting was called to order at $6: 14 \mathrm{pm}$ by Dan Forster at PGHS in the ag department.

7. Angela moved to accept the minutes from the previous meeting as read and Lynn seconded the motion. The motion passed by voice vote.

8. Sharing ldeas of Ag Program names

a. Zallo's Animal \& Anatomy classes had a class assignment to come up with a acronym/brand name for the ag department/program

b. Angela recommended that we look at other states for names of their academy programs.

c. It was suggested to possibly add science into the final program name.

9. Future of $\mathrm{Ag}$ Funding

a. Each student had an assignment in class to write to their local legislators and to find their representatives. They were sent to governor, senator and assembly member. Steinberg responded to students wrote to students telling them they should talk to their district about the funding.

b. Capitol Ag Day - discussion with cut flowers association to possibly make appointment with governor or office staff when floral arrangement is delivered.

c. Lynn suggested that as a group the ag high schools in the district should get together to propose a plan, set amount per student to ask for the local funding money that is allocated for career technical education.

10. Program Completer Defined -

a. A program completer of the PGHS Agriculture program is defined as a student who completes three (3) years, including their senior year in the program and completes coursework in one of the Career Pathways offered. These students will receive a blue cord to wear at graduation. Students who earn their State FFA Degree will wear the gold FFA sash. Students who complete four (4) years in the Agriculture program will receive a pin to wear for their achievement.

i. Advisory members agreed that this was a clear and acceptable definition.

11. Unfinished Business

a. 19 State FFA Degrees - New PG Record

b. Ag Department Survey -

i. Email Angela the survey to create on Survey Monkey

ii. Add a question clarification question about SAE's describing what category it fits in.

iii. For Grad survey - add question about earning FFA scholarship and able to get a job from being in ag class

12. Meeting was adjourned at $8: 14 \mathrm{pm}$. 


\section{Support Material 33:}

Articulation Agreement with Consumnes River College 


\section{Pleasant Grove High School Agriculture Department}

\section{Support Material 33: Articulation Agreement with Consumnes River College}

Pleasant Grove High School has an articulation agreement with one of our local community colleges, Consumnes River College for Economics in Agriculture and Agribusiness 330.

Students who have taken Economics in Agriculture at Pleasant Grove High School can receive college credit at Consumnes River College. This allows our students to be a step ahead of other students at our local community college, which is extremely important as our community colleges continue to experience serious impaction and courses are difficult for students to obtain. 


\section{HIGH SCHOOL TO COLLEGE CAREER PATHWAYS HIGH SCHOOL COURSE ARTICULATION AGREEMENT RENEWAL}

High School District: Discipline:
Elk Grove Unifled

Agribusiness
High School Name:

General Course Title:
Pleasant Grove

Economics in Agriculture

Completion of this form is required by Los Rios Community College District Board Policy/Regulations every two years. It is to be completed by the High School teacher who is teaching the articulated course, as well as the CRC faculty member.

\section{Please review the attached Articulation Agreement and High School Course Outline on record at the College \\ 2. Please print or type all information (excluding instructors' signature boxes)}

Academic Year of This Renewal: 2012-2013

\section{The Following Information Should Be Completed by High School/ROP Instructor:}

HS/ROP Course Title: Economics in Agriculture

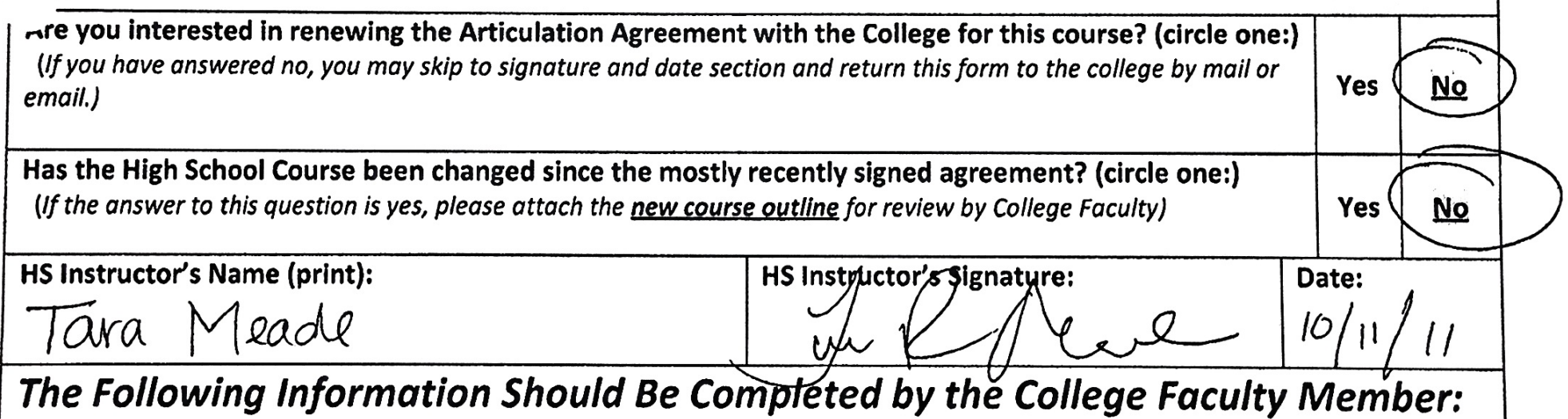

\begin{tabular}{|l|l|l|}
\hline College Course Title: & $\begin{array}{l}\text { College Course Number: } \\
\text { Intro to Agriculture Business }\end{array}$ & College Units: \\
\hline Faculty Facilitator's Name (print): & Signature: & Date: \\
Howard Lewis & Atowarel & $12-14-1 /$ \\
\hline
\end{tabular}
Verification that this renewal has been reviewed by the Curriculum Committee:

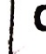$$
\text { L }
$$

Note: Renewal is required every two years; the next renewal is two years from the date signed by Curriculum Chair. 
Q.

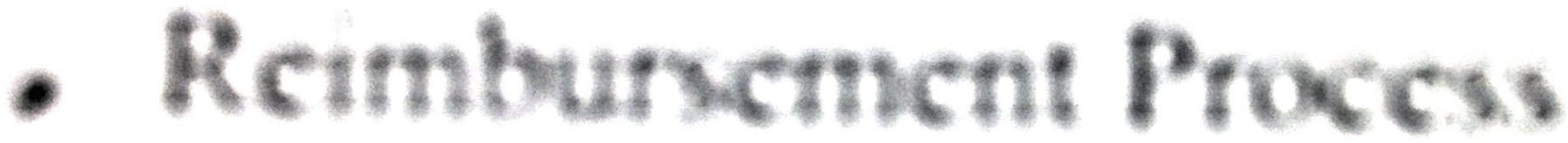


$c$

Support Material 34:

- Reimbursement Process 


\section{Pleasant Grove High School Agriculture Department}

\section{Support Material 34: Reimbursement Process}

The reimbursement process for the Elk Grove Unified School District is outlined through the purchasing office. District (Perkins, Agriculture Incentive Grant, and district funds) reimbursement completed using separate paperwork from the ASB reimbursement process.

District Purchase Orders must be set up in advance, at the beginning of each school year, and must be approved by the Departmetn Chair, Site Controller, and Principal. If purchases are made using a Purchase Order, a reimbursement form with correct supporting documents must be submitted to the District Office.

ASB accounts can also have Purchase Orders, which must be established at the beginning of each school year. When a purchase is made through the FFA account, it must be approved by the FFA Executive Committee and an ASB Representitive, and then a reimbursement claim must be completed and submitted with a supporting receipt to our school's Site Controller. 


\section{Pleasant Grove High School \\ Associated Student Body}

Request for Reimbursement

To be used only when the ASB account being charged has an open purchase order in place.

PLEASE ATTACH RECEIPT(S) TO THE BACK OF THIS FORM

AUTHORIZED ASB PO \# PG

DATE:

(Name of the person to whom the check will be written)

ASB ACCOUNT

ASB ACCOUNT NAME:

NUMBER:

ASB ACCOUNT ADVISOR SIGNATURE:

\section{INDIVIDUAL INVOICE/RECEIPT INFORMATION}

you have more than one receipt please list the information from each receipt on a separate line.)

Receipt

Date

Description of Item(s) Purchased

Reimbursement

Amount

JTAL REIMBURSEMENT REQUESTED:

h. eimbursement is for supplies purchased for a workshop/meeting/conference, etc. you must attach a py of the agenda.

zase complete ALL of the above information and attach your receipt(s) and agenda (if required) to the ck of this form and then submit it to the School Site Controller for reimbursement. 


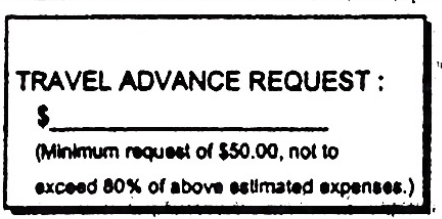

DIRECT PAYMENT REQUEST:

$$
\square \text { YES DNO }
$$

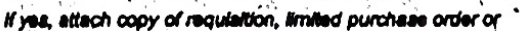

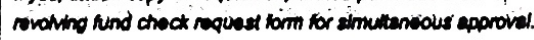

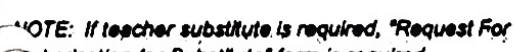
Crontzation tor Subettuto 1 rom is roquirod.

PART B: TO BE COMPLETEO UPON RETURN

ITEMIZED EXPENSES (LWT day and dolo. e.9. Mon. 1/11)

\begin{tabular}{|c|}
\hline TTEM \\
\hline $\begin{array}{l}\text { BREAKFAST } \\
\text { MexImum } \$ 11.00\end{array}$ \\
\hline LUNCH Meximum $\$ 16.00$ \\
\hline
\end{tabular}

OMNWER ...

Maximum $\$ 29.00$

PERSONAL CAR.

MILES Q CURRENT RATE

LODGING: HOTEL. ETC.

CONVENTON REGISTRATION FEES

OTHER - ITEMIZED

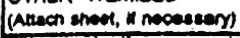

TOTAL ITEMIZEO EXPENSES (Should not OXCood osUmato in ParA A)

IES6 PAYMENTS BY DIGTRICT:

Travel Adrance - Revolving Cash Fund Chock"

DAIE

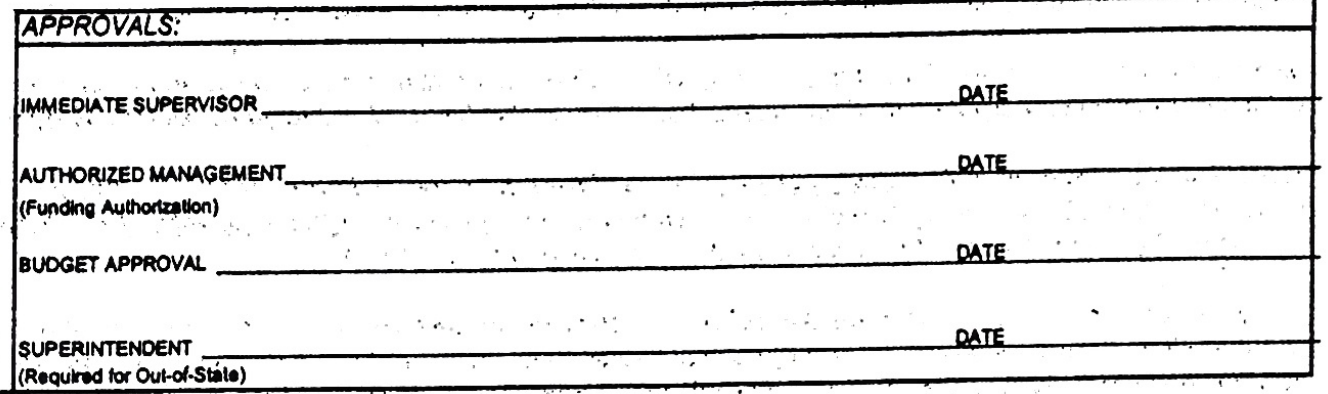

\section{AGENDA ANO APPROPRIATE RECEIPTS MUST BE ATTACHED.}

Direct Payments (From Part B, Column 1)

REIMBURSEMENT DUE

PART C: TO BE SIGNED AFTER EXPENSES ARE ITEMIZED AND AUTHORIZES PAYMENT OF CLAIM.

If dring liability and property damage insurance required by the Stote of Colifomia Venicle Code Soction 16430). I further certify that the mileage and expenses hereon are actual, were expended in the performance of official Gy the Unified District business and that no other claim has boen made for any portion thereof.

SIGMATURE OF EMPLOYEE DATE 


\section{Elk Grove Unified School District MONTHLY MILEAGE CLAIM}

Name

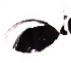

\begin{tabular}{|c|c|c|c|c|}
\hline $\begin{array}{c}\text { FUND } \\
x x\end{array}$ & $\begin{array}{c}\text { SITE } \\
x x x\end{array}$ & $\begin{array}{c}\text { MANAGEMENT } \\
x x x x\end{array}$ & $\begin{array}{c}\text { FUNCTION } \\
x x x x\end{array}$ \\
\hline & & & & \\
\hline & & & & \\
\hline & & & & \\
\hline
\end{tabular}

Address

\section{Approvals: \\ Program Manager (Funding Authonzation) Budget Approval}

\begin{tabular}{l} 
GOAL \\
$x \times x x$ \\
\hline
\end{tabular}

\begin{tabular}{c|c|c|c}
\hline $\begin{array}{c}\text { RESOURCE } \\
x \times x X\end{array}$ & $\begin{array}{c}\text { YR } \\
\mathrm{X}\end{array}$ & $\begin{array}{c}\text { OBJECT } \\
\mathrm{XXXX}\end{array}$ \\
\hline & & & \\
\hline & & \\
\hline & & \\
\hline
\end{tabular}

\begin{tabular}{|l|l|}
\hline AMOUNT & $\begin{array}{c}\text { REIMBURSEMENT DUE } \\
\text { ACCOUNTING USE ON(Y) }\end{array}$ \\
\hline & \\
\hline & \\
\hline
\end{tabular}

Date

Date

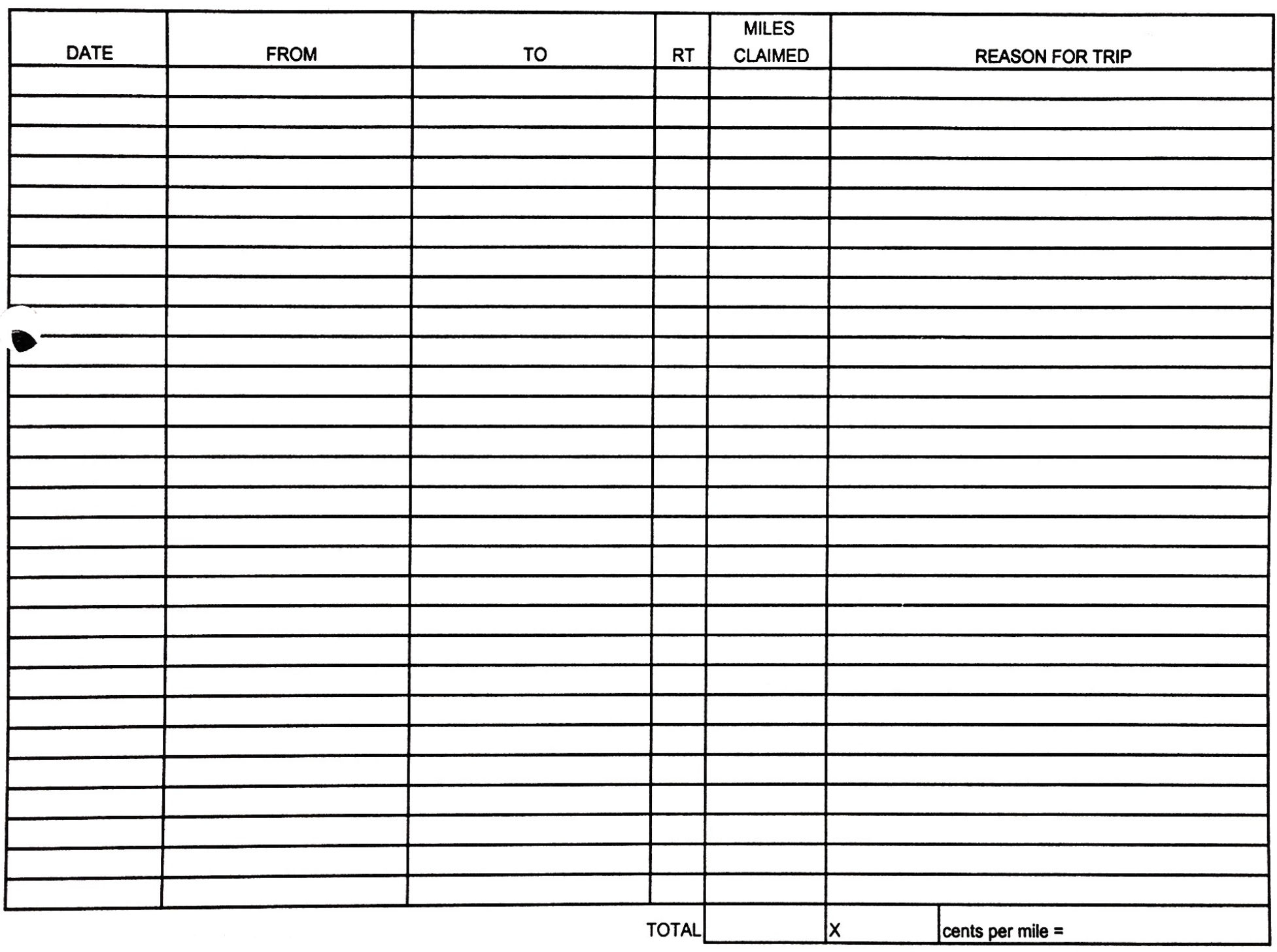

I hereby certify that I possess a valid Califomia Driver's License and that I have sufficient public liability and property damage insurance at least equal to the requirements of the financial responsibility laws of the State of California (Vehicle Code Section 430). I further certify that the mileage and expenses hereon are actual, were expended in the performance of official Elk Srove Unified School District business, and that no prior claim has been made for any portion thereof. 


\section{Section III}

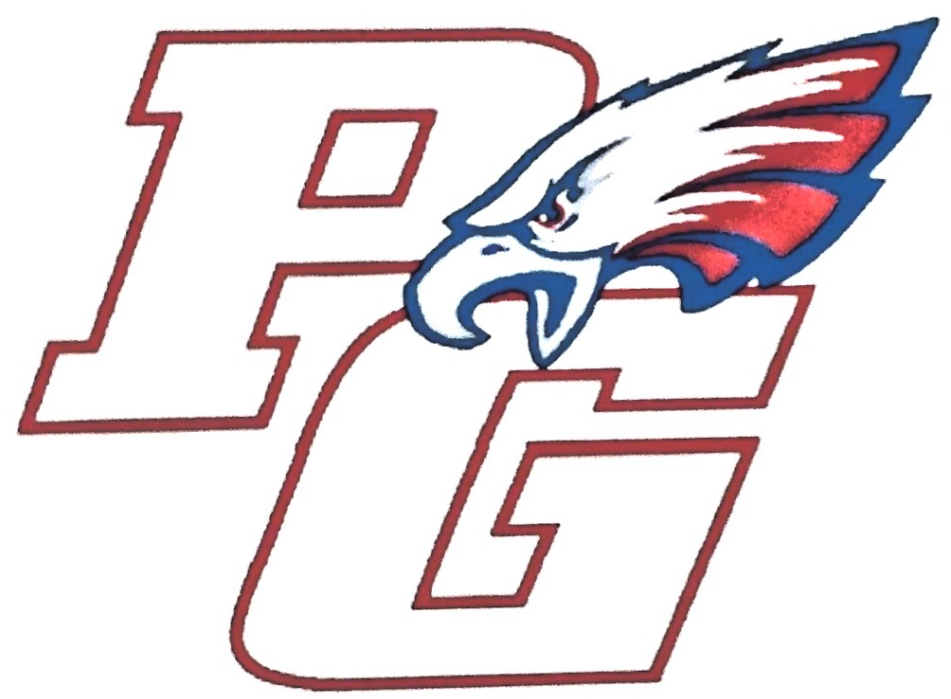

Home of the Eagles

AGED 539 Project 


\section{Table of Contents}

\section{Section III: AGED 539 Project}

AGED 539 Project Description

AGED 539 Program of Activities 
AGED 539 Project: Description 


\section{Pleasant Grove High School Agriculture Department}

\section{AGED 539 Project Description}

My AGED 539 project is the development of a Program of Activities for the Elk Grove-Pleasant Grove FFA Chapter. Pleasant Grove High School was constructed in 2005, and when the agriculture department opened, an FFA Chapter was chartered with a very basic Program of Activities. Over the years, this Program of Activities has been lost, and as such we currently to do not have a completed Program of Activities.

The construction of a Program of Activities was assisted by our Executive Committee, and while this year's Program of Activities may be fairly basic, it is my hope that next year's Chapter Officer Team will begin editing this version of the Program of Activities over the summer to ensure a more updated version for the 2014-2015 school year. 
AGED 539 Project:

2

Program of Activities 


\section{Elk Grove - Dleasant Grove FIA}

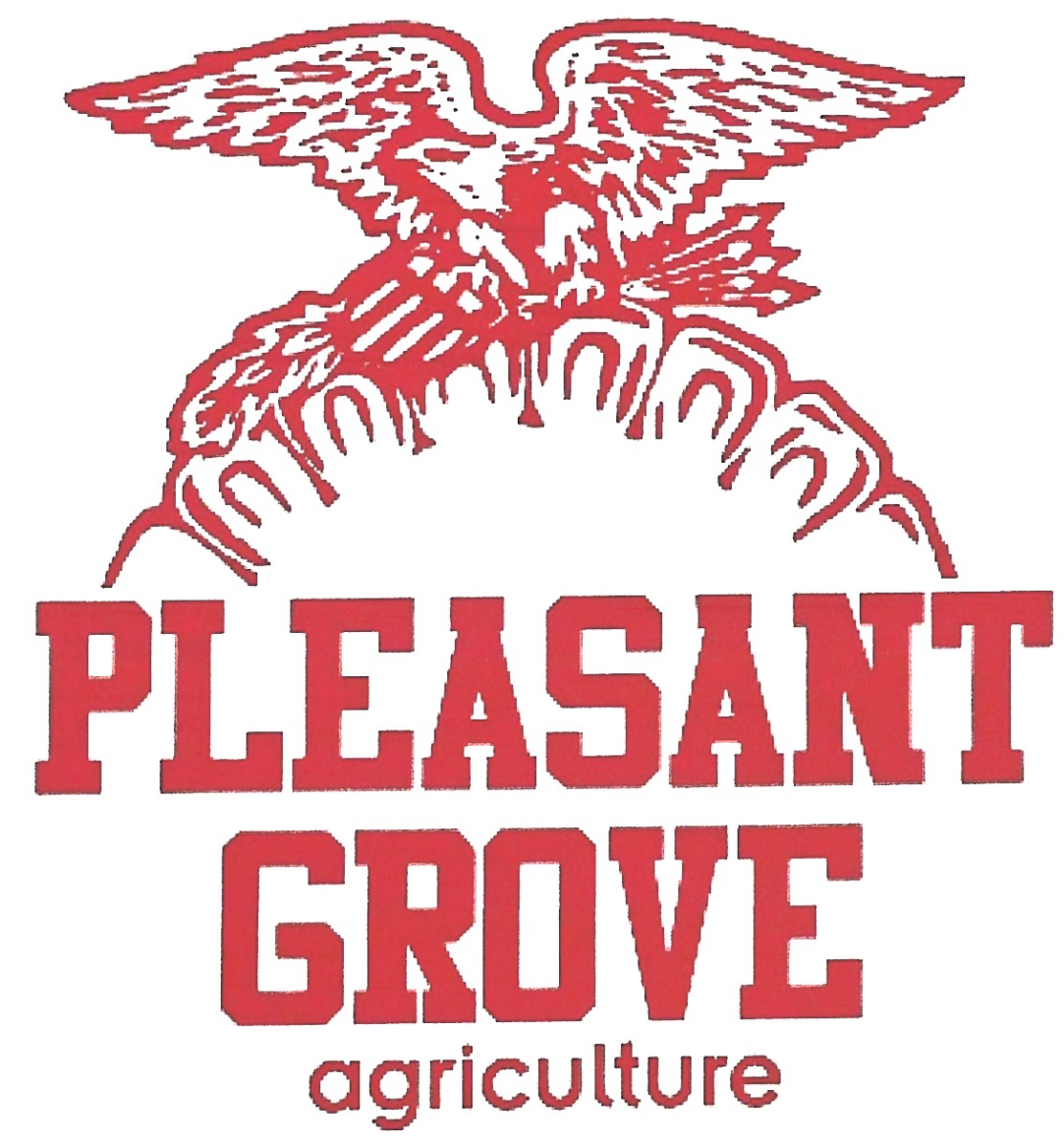

$2013-2014$

Po.

\section{Drogram of Activities}


Table of Contents

esident's Message

2013 - 2014 Chapter Goals

Calendar of Activities

FFA and Agricultural Education

FFA Mission and Strategies

FFA Emblem

\section{8}

FFA Creed

FFA Colors and Motto

FFA Official Dress

FFA Code of Ethics

SAE 13

Cooperation

Leadership

Earnings and Savings

Conduct of Meetings

holastic Achievements and Scholarships

Recreation

Public Relations

Alumni Relations

Fairs and Official Show Uniforms

Agriculture Facility Use Contract

Sacramento County Fair Exhibitor Contract

Market Animal Price Disclosure

Market Swine Project Overview

Market Lamb Project Overview

Market Goat Project Overview

Market Steer Project Overview

Market Turkey Project Overview

Market Rabbit Project Overview

Point Awards System

enapter Constitution

Chapter By-Laws 


\section{Dear Chapter Members,}

The school year is in full swing and we begin another year of FFA excellence.

Pleasant Grove FFA is currently in its $9^{\text {th }}$ year, and is beginning to develop a tradition of students' success and character development. One of the ways that we have reached this success is through FFA activities involving teamwork and dedication.

This year's officer team is like a clean slate. Fresh, new and ready to move forward, we look forward to face the challenges and opportunities that this year holds. I speak for the officer team when I say that we are eager to lead our members in our FFA and agriculture program endeavors.

Our organization is 420 members strong. We hold the power to make a difference in our school and our community while upholding the traditions that are so rich within the FFA organization.

This year, our goals are many, but with the support of our incredible membership, we will triumph and succeed

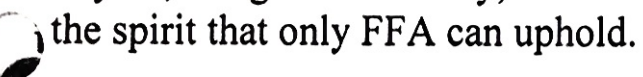

As quoted by legendary football coach Vince Lombardi, "The achievements of an organization are the results of the combined effort of each individual."

The future of our organization will be shaped by the visions that we share with our advisors. I look forward to working with my officer team and fellow FFA members to preserve and enhance our contributions to our school and our community.

Sincerely,

Kelsie Kennicutt

2013-2014 Chapter President 
Our 2013-2014 FFA officer team created the following chapter goals during our annual FFA Chapter Officer etreat held each summer:

\section{Develop Strong and Effective Chapter Leadership}

- Develop and strengthen communication skills

- Increase student involvement $\&$ participation

- $\quad$ Expand leadership classroom resources / supplies

- Improve scrapbook efficiency \& development

- Improve secretary \& treasurer record keeping

- Increase \& improve agriculture leadership curriculum \& resources

- Obtain www.atwaterffa.org web domain

- $\quad$ Upgrade, reformat, and improve current AHS FFA web page

\section{Develop and Strengthen Agriculture Resources and Curriculum}

- Increase large and medium ag mechanics projects

- $\quad$ Expand curriculum technology resources - teaching/learning tools

- Explore reintroduction of ROP Horticulture curriculum

\section{Develop Strong Recruitment \& Retention Program}

- Maintain middle school relationships \& communication

- Maintain / improve recruitment presentations \& education

- Increase retention of students moving into $11^{\text {th }}$ and $12^{\text {th }}$ grade 
2013-2014

PLEASANT GROVE FFA CALENDAR

AUGUST

15 School Begins!!!

27 Farm Clean Up, 3:30 - 5pm

29 New Student Open House, 5:30-6:30pm @ Ag Dept.

SEPTEMBER

3 Labor Day (No School)

$4 \quad A G$ Olympics \& BBQ (5:45pm) @ Cafeteria

18 Movie Night @ 6:00pm, Cafeteria

OCTOBER

1 Greenhand Conference @ Lodi HS

3 October FFA Meeting @6:30pm, Cafeteria

5-6 Harvest Festival @ Elk Grove Park

5-6 Chapter Officer Leadership Conference @ Denair

9 Food Faire \#1

11 End of 1st Quarter

12 Aggie Backers' Dinner/Auction

22 Rough Drafts for Speaking Contests due to FFA Advisor!

23 "Pumpkin Carving Contest" @ 3:45pm @ AG Facility

29-2 National FFA Convention @ Lousiville, KY

NOVEMBER

6 Greenhand Ceremony @ 6:30pm @ Cafeteria

7 S/S FFA Opening/Closing Ceremonies @ 8am, Galt HS

11 Veterans' Day (No School)

13 Food Faire \#2

19 Judging Team Interest Meeting

20 Mandatory County Fair Information Meeting @ 6pm, Cafeteria

25-29 Thanksgiving Break!

DECEMBER

10 S/SFFA Administrator Night @ 6pm

11 December FFA Meeting @6:30pm, Cafeteria

16 FFA Movie Night @4:30pm

18-20 Final Exams! (Minimum Days)

20 End of 1st Semester!

23-6 Winter Break!

JANUARY

$1 \quad$ New Year's Day!

7 School Resumes

10-11 Made For Excellence/Advanced Leadership Academy

15 January FFA Meeting @ 6:30pm, Cafeteria

20 Martin Luther King, Jr.'s Birthday

21 Final Drafts for Speaking Contests due to your AG Teacher!

29 Food Faire \#3

29 S/S FFA Speaking Contests @ 3:30pm, EGHS 
$4 \quad$ Central Region Proficiency Award Scoring @ Ripon HS @ 4pm

5 "Feel The Lovel" @ 6:30pm @ Cafeteria

10 Central FFA Region Officer Interviews @ Modesto JC

10 Lincoln's Birthday (No School)

12 Central Region FFA Speaking Contests Pre-lims @ Galt HS

16-22 National FFA Week!

17 Presidents' Day (No School)

18 Donkey Basketball Game vs. Elk Grove FFA

22 Central CATA/FFA Spring Regional Meeting @ Modesto JC @ 8am

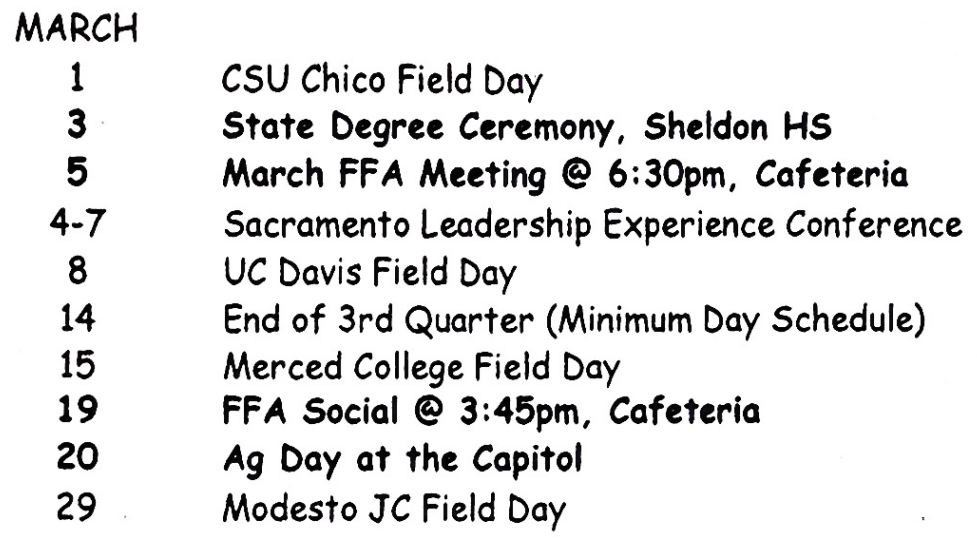

APRIL

2 Chapter Degree Ceremony @ 6:30pm, Cafeteria

5 Cosumnes River College Field Day

9 FFA Social, 3:45pm (tentative date)

9-10 Speech Finals (CSU Fresno)

12 CSU Fresno Field Day

12-15 State FFA Leadership Conference @ Fresno

14-18 Spring Break

MAY

2-3 State FFA Judging Finals @ Cal Poly, SLO

7 FFA Movie Night @ 5:30pm, Cafeteria

8 Sac. Section Planning Meeting / FFA Sectional Officer Elections @ CRC @ 4-6pm

14 FFA Spring Banquet @ PGHS

20-26 Sacramento County Fair

28-30 Finals!!! (Minimum Day Schedule)

* Items on the calendar may change during the school year.

Check with your ag. instructor for updates! 
When you put on an FFA jacket, you become part of a total agriculture education program that will connect you to exciting careers in the science, business and technology of agriculture. FFA is only one of three essential components of this system, all of which work together to provide you with the personal, academic and career experiences essential for your success. Get to know the "three circles" that make this possible.

Classroom/Laboratory Instruction- Agriculture is rooted in science, math, business and technology. The time you spend in the classroom and school lad with you teacher will help you explore and master the information necessary to move forward with you career development. Get ready for exciting hands-on opportunities that make textbooks come alive!

Supervised Agricultural Experience (SAE)- Nothing takes your skills to highest level faster than putting them into practice. Through an SAE, you can create your own landscaping business, conduct a scientific research project that could change the world, grow crops or raise livestock, secure a meaningful job that provides insider experience related to your career choice, or learn how to make a difference in your community though civic engagement. Best of all, you can earn while you learn.

FFA- As an FFA member, you'll work on developing your potential for premier leadership, personal growth id career success. By participating in competitions, degree programs, state and national conventions, community service projects, summer camps and chapter committees, you'll grow in ways that take advantage of your talents and help you become the leader you were meant to be. The key to success in FFA is to get involved!

Make sure you're getting a complete Agricultural Education experience, and remember that it all works together. Talk with you agricultural teacher today and make plans to perform in all three arenas. Don't just settle for a high school diploma when you can get set for life.

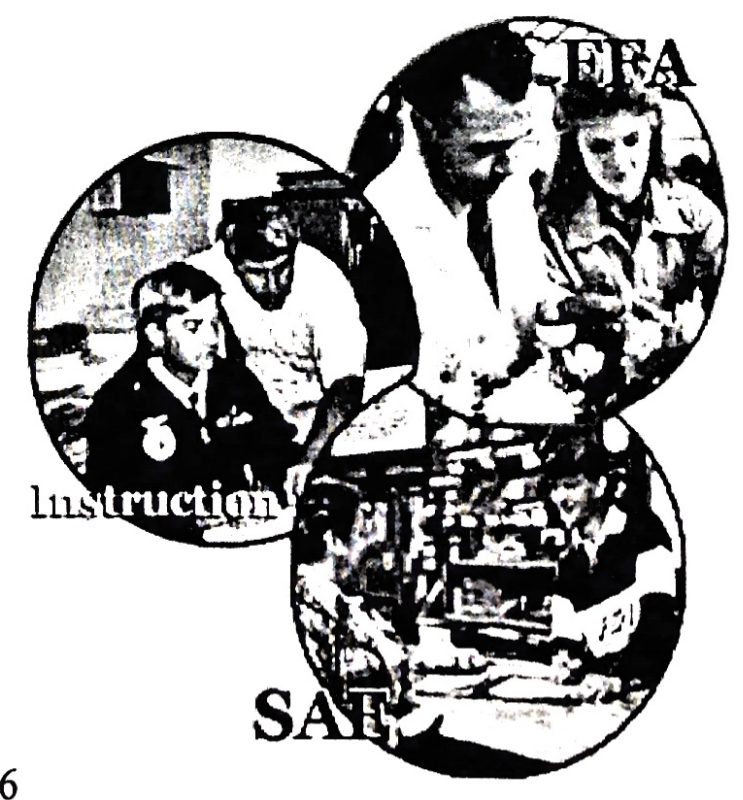


FA makes a positive difference in the lives of students by developing their potential for premier leadership, personal growth and career success through agriculture education.

To accomplish this mission, FFA:

Develops competent and assertive agriculture leadership

Increases awareness of the global and technological importance of agriculture and its contribution to our well-being.

Strengthens the confidence of agriculture students in themselves and their work.

Promotes the intelligent choice and establishment of an agricultural career

$>$ Encourages achievement in supervised agricultural experience programs

$>$ Encourages wise management of economic, environmental and human resources of the community

Develops interpersonal skills in teamwork, communications, human relations and social interaction.

> Builds character and promotes citizenship, volunteerism and patriotism.

> Promotes cooperation and cooperative attitudes among all people.

$>$ Promotes healthy lifestyles.

Encourages excellence in scholarship.

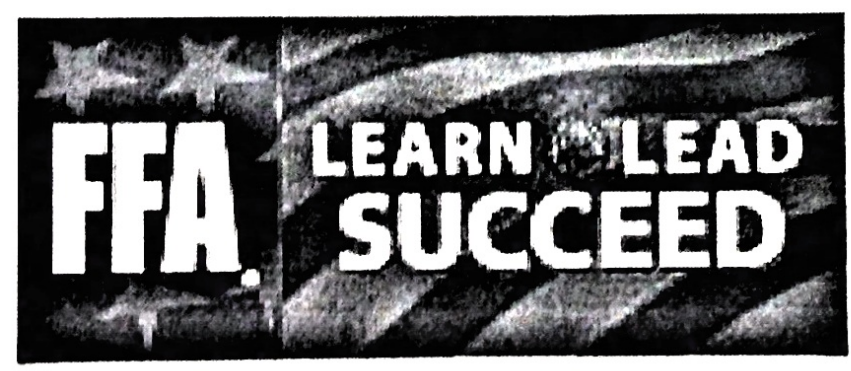


Yany organizations have logos they use as part of their identity. As with most logos, the FFA emblem is symbolic. It contains five separate elements. Each element represents items or ideals that are important to the organization and its members.

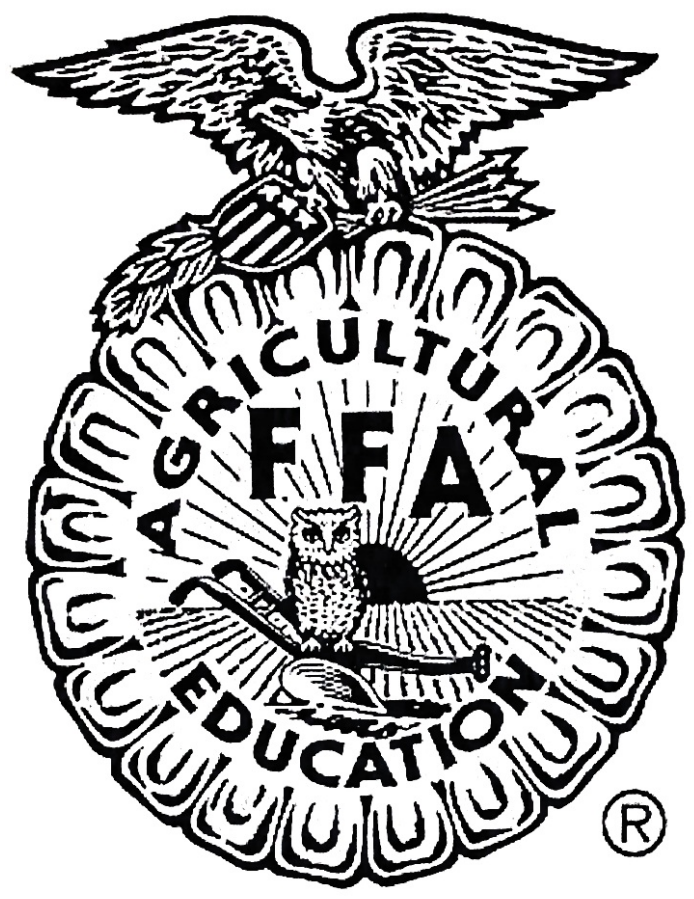

The cross-section of an ear of corn serves as the emblem's foundation, just as corn has historically served as a foundation crop in American agriculture. Corn is also a symbol of unity because it is native to America and it is grown in every state.

The rising sun appears in the center of the emblem and symbolizes progress in agriculture and the confidence FFA members have in the future.

The plow is a symbol of labor and tillage of the soil.

The owl represents knowledge and wisdom.

The eagle is perched on top of the emblem and served as a reminder of our freedom and ability to explore new horizons for the future of agriculture.

Finally, the words, "Agriculture Education" surrounding the letters "FFA" indicate that the FFA is an important part of the agricultural education program. 
The FFA Creed is a basic statement of beliefs and a common bond between members. The creed was written by E.M. Tiffany and adopted at the $3^{\text {rd }}$ National FFA Convention. It was revised at the $38^{\text {th }}$ and $63^{\text {rd }}$ conventions to reflect changes in FFA members and the agricultural industry.

\section{The FFA Creed}

I believe in the future of agriculture, with a faith born not of words but of deeds - achievements won by the present and past generations of agriculturists; in the promise of better days through better ways, even as the better things we now enjoy have come to us from the struggles of former years.

I believe that to live and work on a good farm, or to be engaged in other agricultural pursuits, is pleasant as well as challenging; for I know the joys and discomforts of agricultural life and hold an inborn fondness for those associations which, even in hours of discouragement, I cannot deny.

1 believe in leadership from ourselves and respect from others. I believe in my own ability to work efficiently and think clearly, with such knowledge and skill as I can secure, and in the ability of progressive agriculturists to serve our own and the public interest in producing and marketing the product of our toil.

I believe in less dependence on begging and more power in bargaining; in the life abundant and enough honest wealth to help make it so--for others as well as myself; in less need for charity and more of it when needed; in being happy myself and playing square with those whose happiness depends upon me.

I believe that American agriculture can and will hold true to the best traditions of our national life and that I can exert an influence in my home and community which will stand solid for my part in that inspiring task.

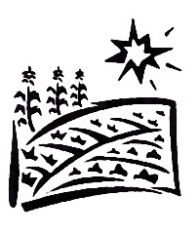




\section{Colors}

The National FFA Organization chose national blue and corn gold as its official colors in 1929. As the blue field of our nation's flag and the golden fields of ripened corn unify our country, the FFA colors give unity to the organization.

\section{Motto}

Many important things come in small containers. Although a diamond ring takes up a little space, it is extremely valuable. So it is with the FFA motto. The motto has just 12 words, but those words are powerful.

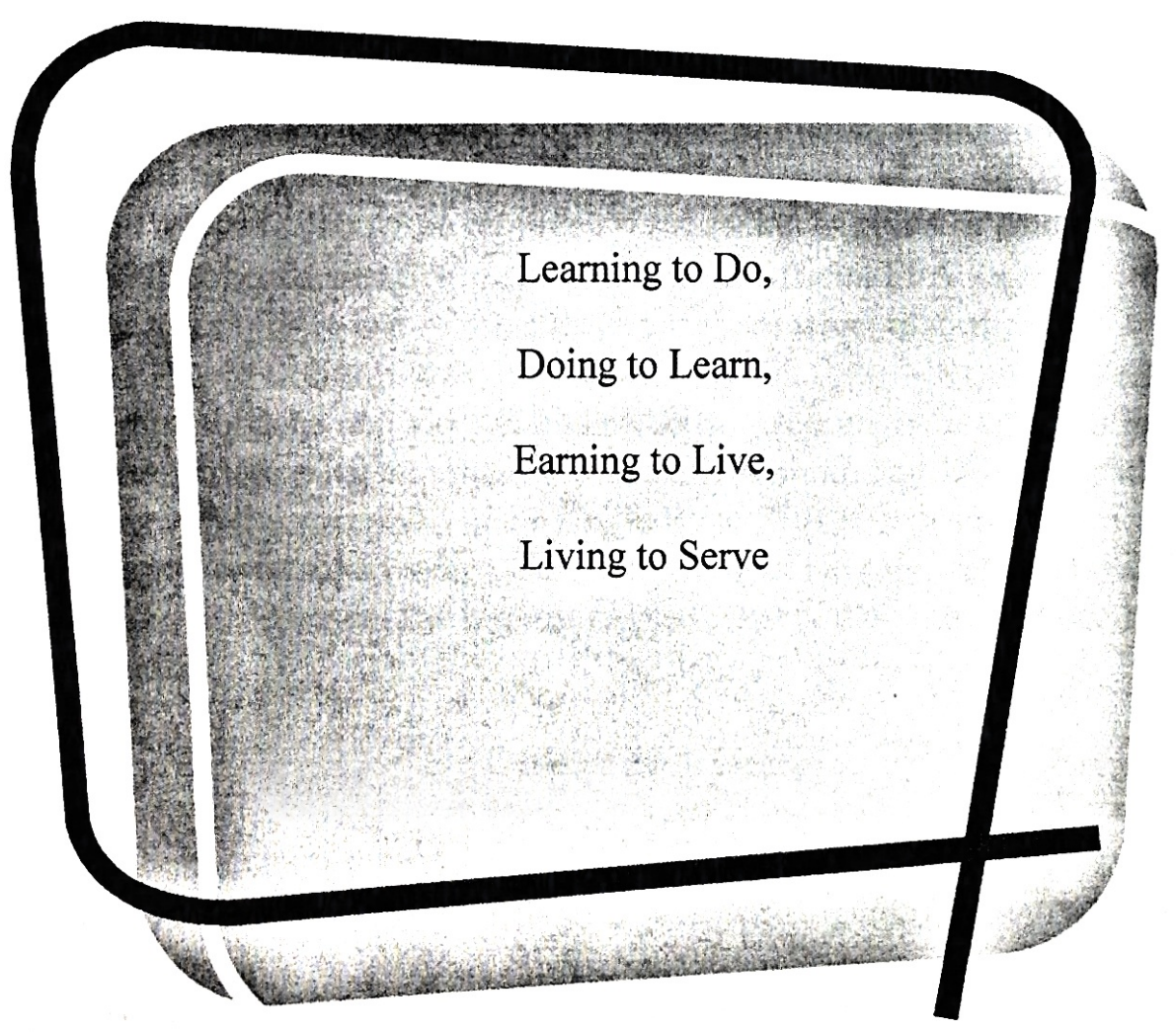


One of the most unifying elements for any group is its uniform. In FFA, the uniform members wear to local, Tate and national functions is called official dress. It provides identity and gives the organization a distinctive and recognizable image.

\section{Proper Use of the FFA Jacket}

- The jacket is to be worn only by members.

- The jacket should be kept clean and neat at all times.

- The back of the jacket includes only: a large official FFA emblem, the name of the state association and the name of the local chapter, district or area. The front of the jacket includes only: a small official FFA emblem, the name of the individual, one office or honor and the year of that office or honor.

- The jacket should be worn on official occasions with the zipper fastened to the top. The collar should be turned down and the cuffs buttoned.

- The jacket should be worn by members and officers on all official FFA occasions, as well as other occasions where the chapter or state association is represented. It may be worn to school and other appropriate places.

- The jacket should only be worn to places that are appropriate for members to visit.

- School letters and insignia should not be attached to or worn on the jacket.

- When the jacket becomes too faded and worn to wear in public, it should be discarded or the emblems and lettering should be removed.

- The emblems and lettering should be removed if the jacket is given or sold to a non-member.

- A member should act professionally when wearing the official FFA jacket.

- Members should refrain from use of tobacco and alcohol when underage and at all times when representing the FFA. In addition, members should exhibit their leadership qualities when they encounter substances including tobacco and alcohol and serve to discourage others from inappropriate behavior.

- All chapter degree, officer pins, and other award medals should be worn beneath the name on the right side of the jacket, with the exception that a single State FFA charm and American FFA key should be worn above the name or attached to a standard key chain. No more than three medals should be worn on the jacket; these should represent the highest degree earned, the highest office held and the highest award earned by the member.

\section{Official FFA Dress}

- Official dress for female members is a black skirt, white blouse with blouse with official FFA scarf, black shoes, and official jacket zipped to the top. Black slacks may be worn for traveling and outdoor activities.

- The official dress for male members is black slacks, white shirt, official FFA tie, black shoes, black socks and the official jacket zipped to the top. 
Reople are always observing you. Your actions when you wear the FFA jacket or represent the organization become part of the organization's image. To keep the image of the FFA and members sharp, delegates at the 1952 National FFA Convention adopted a Code of Ethics for FFA members to follow. The FFA Code of Ethics still protects the FFA image. It also guides members to make positive, healthy choices - and not only during FFA activities. The code of ethics guidelines are good to follow during all occasions and functions.

\section{The FFA Code of Ethics}

FFA Members conduct themselves at all times to be a credit to their organization, chapter, school, community and family. I pledge to:

Develop my potential for premier leadership, personal growth and career success

Make a positive difference in the lives of others.

Dress neatly and appropriately for the occasion.

Respect the rights of others and their property.

Be courteous, honest and fair with others.

Communicate in an appropriate, purposeful and positive manner.

Demonstrate good sportsmanship be being modest and winning and generous in defeat.

Make myself aware of FFA programs and activities and be an active participant.

Conduct and value a supervised agricultural experience program.

Strive to establish and enhance my skills through agricultural education in order to enter a successful career.

Appreciate and promote diversity in our organization. 
What if you could get classroom credit and FFA awards for doing what you like: experimenting with careers, earning money, building a resume and having fun? You can - with a Supervised Agricultural Experience (SAE program. An SAE is a program you design to gain hands-on experience and develop skills in agricultural careen areas that interest you.

You choose an SAE program that lets you discover, explore, experience and excel in careers. In the meantime, you gain skills and experience that pay off in areas of life. Your SAE program can lead you toward personal growth, premier leadership, and career success.

An SAE program is not just another class assignment or graduation requirement. You are truly in charge of you SAE! Although your agriculture teacher will help you learn related information and keep good records, the success or failure of your SAE is up to you. It's an exciting opportunity to prove your abilities to future employers - ana to yoursell.

\section{Pleasant Grove FFA SAE Program}

* All Chapter Members are required to maintain a Supervised Agriculture Experience (SAE) program.

* Members are encouraged to apply for local, regional and state proficiency awards.

Members are encouraged to apply for advanced degrees (i.e. State FFA Degree)

* Members are encouraged to compete in the Local and Sectional Project Competition.

Members are required to follow project Advisor's recommendations concerning their SAE.

Members are encouraged to strive to improve and develop their SAE each year.

- Encourage members to develop skills within their SAE through participation and appropriate judging teams.

- Members are encouraged to attend demonstrations, breeding shows, and equipment shows which will enable them to increase their efficiency and knowledge of their SAE.

* Members are encouraged to provide support and help their fellow Chapter members.

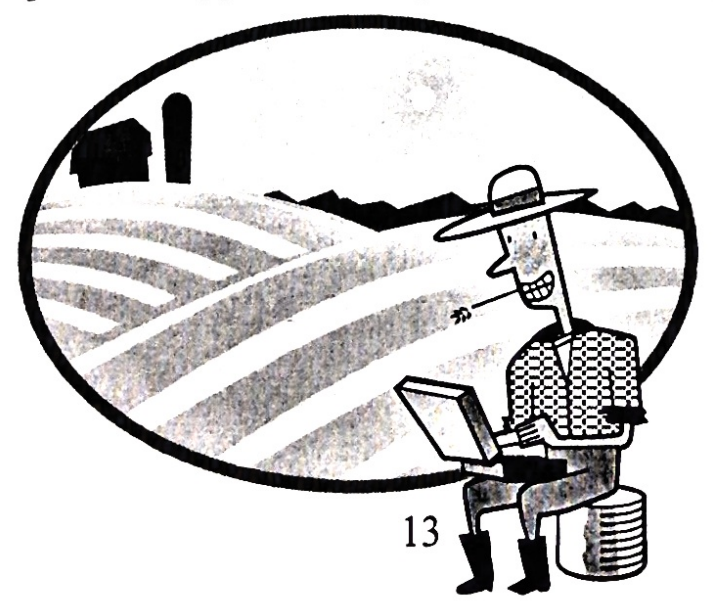


The Pleasant Grove FFA chapter will develop a sense of cooperation among the entire membership.

- The Pleasant Grove Chapter will cooperate with other FFA chapters.

- Participation in Sectional, Regional, and State activities.

- Hosting Sectional activities as needed.

- The Chapter will cooperate with Pleasant Grove High School.

- Assist with ticket sales during home PGHS football games.

- Participation in school functions and events

- Chapter representation during school sponsored activities and functions

- Cooperation and support with other PGHS youth organizations

- The Chapter will participate in community cooperation.

- Participating and working with Elk Grove Community Services District at community events.

- Providing local middle schools with an informative recruitment presentation.

- Participation in at least three community service project per year as coordinated by the FFA Officer Team

- Members exhibiting at fairs and shows will cooperate together and compete as a team. 
Leadership is the ability to guide or influence others to work towards a meaningful goal while helping each to Lvelop themselves as group members. Leadership is the ability in a well-adjusted person to handle people, to inspire or influence the actions of others, to make decisions or to move a group to action. Leadership is a contribution to the establishment and attainment of group processes. Therefore, leadership is a quality of group action.

\section{Public Speaking}

- Creed Speaking Contest

- Impromptu Speaking Contest

- Prepared Public Speaking

- Extemporaneous Public Speaking

- Parliamentary Procedure

- Job Interview Contest

- Opening and Closing Ceremonies Speaking Contest

\section{Committees}

- Every member is encouraged to be an active member of at least one committee or involved in some kind of activity

- Select Chapter members as Co-Chairs for Committees to work effectively with officer to achieve the committee's goals

\section{Degrees and Awards}

- Encourages every member to apply for Greenhand and Chapter Farmer FFA Degree Encourage every qualified member to apply for the State and American FFA degree

- Encourage members to apply for State Proficiency Awards

\section{Officer / Leadership Training}

- Annual Chapter Retreat for new officers

- Leadership Training Conference for all officers

- Sectional \& Regional Officer Training

- Made for Excellence Leadership Training / Advanced Leadership Academy / Sacramento Leadership opportunities available to all members

\section{Meetings}

- Conduct meetings in an orderly fashion by utilizing Parli-Pro

- Have regularly scheduled Executive Meetings and Chapter Meetings

- Encourage every member to attend and participate at all meetings

- Send delegates to all Sectional, Regional, and State meetings

\section{Offices}

- Encourage local members to run for local, sectional, regional, and state offices

- Invite Sectional, Regional, and State Officers to speak to your chapter 
As a non-profit organization, the earnings and savings aspect of our chapter is very important towards the lccess and productivity of our 2013-2014 school year.

The chapter earns money in various ways in order to finance FFA events and activities throughout the year. Some of these activities include:

- Sale of apparel and lanyards

- Fat Mike's Restaurant Fundraiser

- $\quad$ Assistance with chapter needs via PGHS Aggie Backers

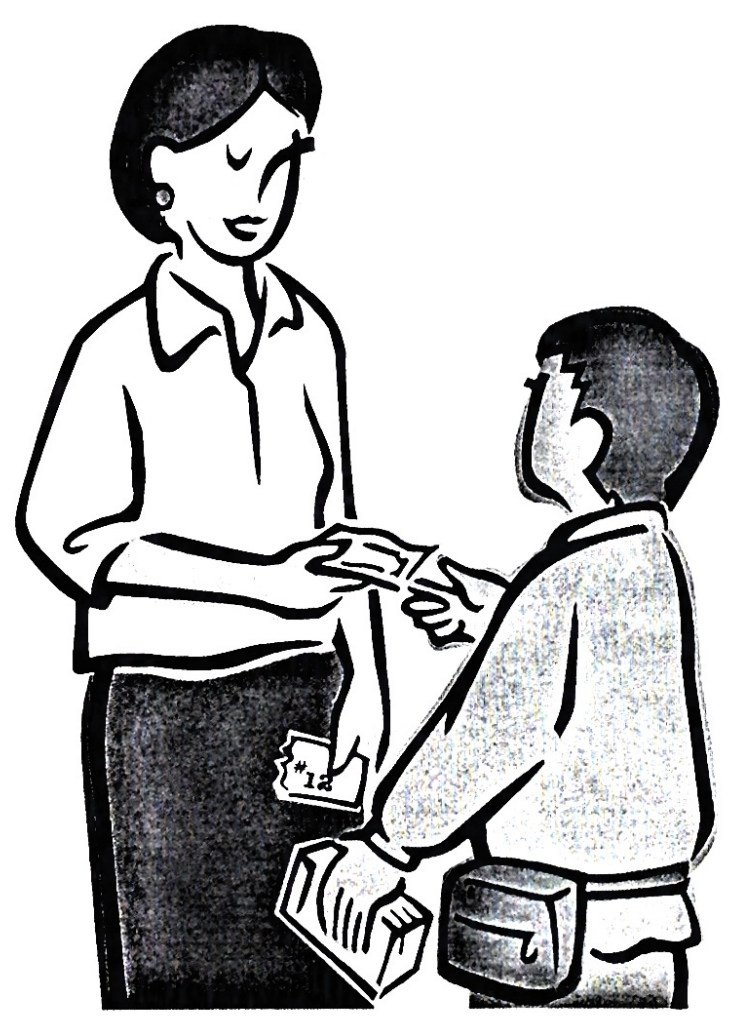




\section{Conduct of Meetings}

Hold Regular, Well-Planned Meetings that Capture the Chapter's Interest and Participation

- Have weekly Chapter Officer Meetings

- Have regular month Chapter Meetings

- Call special meetings when necessary

- Conduct regular Executive Meetings in order to maintain solid Chapter communications

- Prepare a well planned program before meetings

- Have frequent and informative committee reports

- Invite parents and the community leaders to the Chapter Meeting

- The duty of the Sentinel is to set the proper paraphernalia out for the Chapter Meeting and to help the President in maintaining order.

- The goal of the Chapter is to have a least $40 \%$ attendance at each Chapter Meeting

- Have an activity after every Chapter Meeting

\section{Special Meetings Should be Held as Necessary}

- Refreshments such as a congratulatory cake will be available at the Greenhand and Chapter Degree Ceremonies to celebrate the achievements of our members

- A very special Awards Banquet will be held in May to wrap up the year.

\section{The Official Ceremonies will be used at all Meetings}

- All officers are required to learn their part.

- The officers will wear the official uniform at all meetings

- The necessary paraphernalia will be used at all meetings.

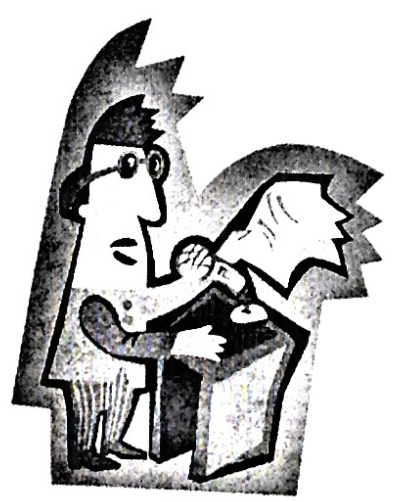


The chapter will encourage students to strive for academic excellence.

\section{Improve scholarship of FFA members in all academic subjects}

A. Encourage members to strive for C.S.F. standards

B. Encourage FFA members to take advantage of the Renaissance system

C. Members participating in competitive events shall not fall below a $\mathrm{C}$ or 2.0 average on a 4.0 scale and no F's as well as no less than a D in any agriculture class. Eligibility for all competitive events will be suspended if any of the above shall occur.

2. Will encourage Seniors to apply for scholarships available to them in order to continue their education.

A. Twelfth grade members are encouraged to apply for the Elk Grove Community Foundation, Farm Bureau, California Women in Agriculture, Young Farmers \& Ranchers, and local Cattlemen's Association Scholarships, which are available to local students.

B. Twelfth grade members are encouraged to apply for any scholarships which are available to them for the school they are planning to attend.

C. Twelfth grade chapter members are encouraged to talk with their counselors about other scholarships which may be available to them as a result of their parent's affiliation with a lodge or places of employment. 
The purpose of recreation is to create an opportunity for FFA members to participate in recreational activities id develop one's social and team building skills. It's also an opportunity to have some fun!

Here's a list of recreational activities scheduled for the 2013-2014 school year:

Each month, members will have the opportunity to attend an FFA Movie Night

September

October

December

March

April

June
Annual Ag Olympics/Welcome Back BBQ

Pumpkin Carving Contest

12 Days of Christmas Activity

St. Patty's Social

Kickball Game

Achievement Trip to 6 Flags, Vallejo 
ie purpose of public relations is to inform our chapter members and the general public about the activities of our local chapter as well as the overall benefits of the FFA.

\section{Media}

- Establish, strengthen, and maintain relationships with media contacts

- Submit articles and photos to various local, regional and state media publications

- Extend media coverage beyond newspapers and publications (i.e. radio, tv, etc...)

\section{FFA Week}

- Use various forms of media to keep the public informed during FFA Week

- Conduct school and community activities throughout FFA Week

\section{Various FFA Events and Activities}

- Select worthy persons as Honorary Chapter farmers

- Host an award recognition ceremony for parents and families of FFA members

- Maintain a chapter scrapbook

- Maintain activity with various community groups

\section{Pleasant Grove High School}

- Regular communication sessions with PGHS administration and counselors

- Assist and support various school activities 


\section{$\underline{\text { Alumni Relations }}$}

The Chapter will encourage graduating seniors to keep membership affiliation for the following year.

- As graduate members, the chapter will encourage these members to:

- Exhibit at fairs until eligibility until membership expires

- Apply for advanced degrees such as the American FFA Degree

- Apply for proficiency awards in their SAE area

- Continue an active role in participation in local activities

- Attend all chapter meetings

- The Chapter will utilize the expertise of alumni members when needed by the Chapter.

- To help coach judging teams

- To serve as judges of local FFA contests

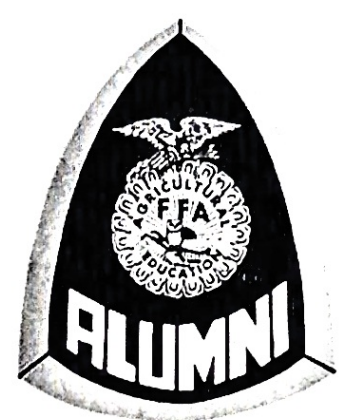




\section{Fairs and Official Show Uniforms}

\section{Official Show Uniform}

The official Show Uniform for FFA members consists of:

White shirt or blouse, white pants, FFA tie, and FFA jacket. Shoes should be appropriate for what you are showing.

\section{Fairs}

The Pleasant Grove FFA Chapter has exhibitors who are involved in many fairs throughout the year. The livestock projects are exhibited throughout the state, where the chapter wins many awards and honors. The animals that are exhibited include market and breeding sheep, market and breeding beef, market and breeding swine, dairy cattle, rabbits, cavies, breeding and market poultry, market, breeding, and dairy goats.

The Fairs that are attended are:

- $\quad$ (spring) Sacramento County Fair

- California State Fair 


\section{Pleasant Grove High School Agriculture Department Agriculture Facility Contract}

Bo proud of tho Ploasant Grovo High School Agriculluro Facillty,

II is a priviloge to raise an animal on these new premises; to onsure the salety of students and animals ALL policies by the Agriculture Department, Pleasant Grove High School and Elk Grove USD will be enforced while at the PGHS Agriculture Facility. In order to keep an animal at the Agriculture Facility, the students must be a Ploasant Grove FFA member in good standing and currently onrolled in a Pleasant Grovo High School Agriculture class.

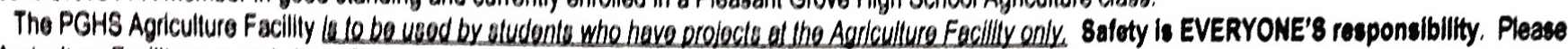
keep the Agriculture Facilly neat and clean, for the benefit of all. Place cleaned tools and equipment in their appropriate location.

In the ovent of an emergency, please call an Agriculture Instructor and a veterinarian. If it is not your animal, contact the owner at the phone number provided. Do not administer medication to another person's animal without approval by the owner and the Agriculture Instructor.

Any darnage to the lacility should be reported to an Agriculture Instructor immediately. Reter to the emergency numbers posted at the Agriculture Facility.

The Agriculturo Dopartmont, Ploasant Grovo FFA, PGHS or EGUSD will not bo hold llable for Injury, loss, damage or theft to you, your livestock or your proporty, which may occur at tho Agriculturo Facility. Studonts and paronts assumo all risks and in addition to the mandatory livestock insurance, should consider pollclos to cover other proporty.

Student with animals on the PGHS Agriculture Facillty are required to attend mandatory cleanup on the Immediate Tuesday after the Sacramento County Fair. The Agriculture Inatructors will Inform students of the schoduled ilmo by the Iirst Monday of May to plan accordingly.

\begin{tabular}{|c|c|}
\hline $\begin{array}{l}\text { Responsibilities of Cooperatively Penned Animals } \\
\text { SWINE, SHEEP \& GOATS }\end{array}$ & $\begin{array}{l}\text { Responsibilities of Individually Penned Animals } \\
\text { STEERS \& REPLACEMENT HEIFERS }\end{array}$ \\
\hline $\begin{array}{l}\text { 1. The responsibility of keeping the pen clean and feeding the animals will } \\
\text { be a cooperative effort by all students in that specific pen. The species' } \\
\text { advisor will develop a feeding/cleaning schedule that will be made } \\
\text { available to students. } \\
\text { 2. A student's project may need to be moved on occasion as conditions } \\
\text { change at the facility. Students will be notified before this occurs by the } \\
\text { Agriculture Instructors. } \\
\text { 3. Hogs, lambs, goats and the feed must be purchased through the } \\
\text { Agricullure Department if the animals will be raised at the school facility } \\
\text { for the health of the livestock brought to the facility. Any unused funds } \\
\text { will be returned to students after the project is completed. } \\
\text { 4. Students are responsible to purchase Livestock Insurance for all market } \\
\text { swine, sheep \& goats kept at the Agriculture Facility. } \\
\text { 5. Students are responsible for keeping track of all purchases related to } \\
\text { their livestock project (e.g. feed, vet supplies, rent, livestock insurance, } \\
\text { equipment) and recording them in their Agriculture Record Book. } \\
\text { 6. All market animals must be removed from PGHS Agriculture facilities by } \\
\text { the opening day of the county fair. }\end{array}$ & $\begin{array}{l}\text { 1. The responsibility of keeping the pen clean and feeding the animals } \\
\text { will be that of the owner of the animal. } \\
\text { 2. A student's project may need to be moved on occasion as conditions } \\
\text { change at the facility. Students will be notified before this occurs by } \\
\text { the Agriculture Instructors. } \\
\text { 3. Animals must be approved by the advisor prior to coming into the } \\
\text { facility, Students are responsible for purchasing their own feed for } \\
\text { their cattle. } \\
\text { 4. Students are responsible to purchase Livestock Insurance for all } \\
\text { market steers and replacement heifers. } \\
\text { 5. Students are responsible for keeping track of all purchases related to } \\
\text { their livestock project (e.g. feed, vet supplies, rent, livestock } \\
\text { insurance, equipment) and recording them in their Agriculture Record } \\
\text { Book. } \\
\text { 6. All market cattle must be removed from PGHS Agriculture facilities by } \\
\text { opening day of the county fair. }\end{array}$ \\
\hline
\end{tabular}

\section{WARNINGS}

Cruelty to the animals will not be tolerated and is cause for immediate eviction of the students and their animal project(s) on the 1at offense by the Agriculture Department. This includes but is not limited to: neglect, abusive training, malicious actions, or use of unapproved drugs. Also, the students will not be able to show an animal as a representative of Pleasant Grove FFA at a livestock show for one calendar year. If students have prior school suspensions for destruction of school property/vandalism, they will not be allowed to house their livestock projects at the Agriculture Facility for the safety and security of livestock and facilities.

The Agriculture Instructors reserve the right to make decisions concerning the Agriculture Facility rules/projects and barn in the best interest of the students and livestock at any time. These rules will be accessed, reviewed and amended by the Agriculture Instructors as needed.

a. 1" Offense - Oral Communication to the Student

b. $2^{\text {nd }}$ Offense - Written Communication to the Student and Parent

c. $3^{\prime 0}$ Offense - Eviction of Project within 3 days. If the student's project is not removed after 3 days, then the Agriculture Instructors will sell the animal at a livestock yard facility. Student will receive any proceeds of the sale after any outstanding money owed to the Agriculture Department has been paid.

I agreo to ablde by the Pleasant Grove High School Agriculture Department pollcy while housing my animal(s) at the Agriculture Facility.

Student Signature:

Date:

Parent Signature:

Date: 


\section{Pleasant Grove High School Agriculture Department Sacramento County Fair Exhibitor Contract}

1. Your \#1 priority at county fair is to take care of your animal!!! Make sure that your animal is fed; plenty of water is provided; and make sure your pen is cleaned every morning. Students are expected. to feed their animal(s) and have their pens cleaned before 7:30am every day of the fair.

2. YOU are responsible for caring for your own animal(s). This includes: feeding, fitting, constant water supply available for the animal, cleaning pen area, return tack and equipment to proper location, etc.

3. You will be assigned barn duty shift(s) within your species. This will ensure that the livestock pens are appropriately maintained and the animals are cared for. You will have a minimum amount of shifts to not exceed a two-hour block per day. If you work during fair, you will be assigned accordingly. You will be required to assist in the loading and unloading of animals and supplies/equipment to and from the fair.

4. You will receive your fair check AFTER you have submitted your up-to-date FFA record book and a buyer's thank you letter to your Advisor. Approximately 2-4 weeks after county fair, the checks are sent to your Advisor, who will notify you of when you can pick up your fair check at school.

5. While in FFA uniform, you need to remember the image you are projecting for both yourself and your chapter. Please, make certain that you always look and act appropriately. This includes keeping the jacket zipped to the top and all clothing (including shoes) worn at all times. (Review "FFA Show Uniform \& Public Image" handout). It is strongly encouraged to purchase your own FFA jacket and FFA scarf/tie. See your Agriculture Instructor for assistance.

6. If you will be staying at the campsite or at a hotel, there must be an adult (at least 27 years old) assigned to your tent/trailer/hotel room. This is in accordance with the EGUSD policy for student activities.

7. Be on your best behavior. Behavior that is disruptive or dangerous like use or possession of alcohol or drugs, smoking in barns, defacing property, etc. will result in you and your animal being removed from the Sacramento County Fair. Pleasant Grove High School Policies will be enforced.

8. Your parent/guardian must provide or arrange for your transportation to and from fair each day.

I understand and agree to abide by the above expectations set by the Pleasant Grove High School Agriculture Department. 


\section{Pleasant Grove High School Agriculture Department Market Animal Price Disclosure}

Each species of market livestock carries with it inherit costs associated with the project, which you can find either in your species handbook or the budget presented to you at your individual species meetings. These budgets are an estimate and while we have done our best to estimate costs for the projects, it is important to remember that additional and/or unforeseen costs can occur throughout the course of a project. These include but are not limited to:

- Veterinarian expenses due to illness and/or injury to the animal which is outside of normal preventative veterinary care:

- A basic ranch call to the Pleasant Grove High School Agriculture Department typically starts at a cost of $\$ 150$ - this does not include medication, materials, etc.

- Veterinary costs can grow exponentially depending on the situation - it is recommended that you discuss treatment with your chosen vet prior to agreeing to any treatment.

- Additional show supplies that may be necessary for a specific animal's needs/purposes.

- Feed expenses may also change for individual animals depending on the animal's needs and body condition.

- Entry fees for fair

- These vary greatly depending on the number of classes you enter, how many fair passes you will need, etc.

Due to the nature of the Junior Livestock Auction at the Sacramento County Fair, auction prices (\$ per pound) can vary greatly from year to year and from exhibitor to exhibitor. Your species advisor will provide you with an average price received from Pleasant Grove FFA Exhibitors from the previous year, but this is a guideline only and does not guarantee you will receive that price.

Due to variances in both project costs and Junior Livestock Auction prices at the Sacramento County Fair, the PGHS Agriculture Department would like to make it clear that we cannot guarantee any student will earn a profit on their market animal project. We will however offer as much assistance as possible in assisting students with writing buyer letters, marketing their projects, and finding buyers.

I have read and understand the above statements regarding market animal prices.

Student Signature:

Date:

Parent Signature:

Date: 


\section{-Market Swine}

1. Sample Budget

\begin{tabular}{|c|c|c|}
\hline & \multicolumn{2}{|c|}{ Market Hog } \\
\hline Cost of Animal & $\$$ & 250.00 \\
\hline Feed & $\$$ & 200.00 \\
\hline Show Supplies & $\$$ & 15.00 \\
\hline Entry Fees & $\$$ & 28.00 \\
\hline Veterinary Supplies & $\$$ & 10.00 \\
\hline Livestock Insurance & $\$$ & 17.00 \\
\hline Estimated Expenses & $\$$ & 520.00 \\
\hline Sale of Animal & $\$$ & 720.00 \\
\hline & & $\$ 3.00 / \mathrm{lb}$ \\
\hline Estimated Net Profit & $\$$ & 200.00 \\
\hline
\end{tabular}

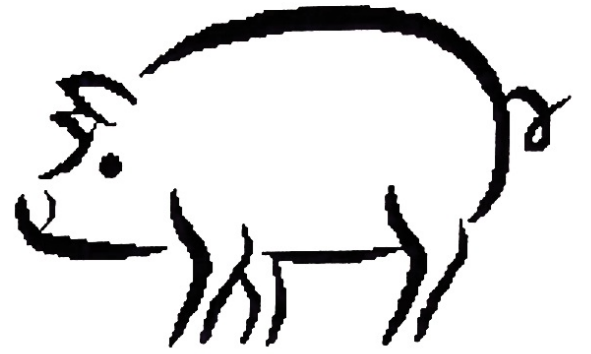

2. Ownership of Project

- Sacramento County Fair is May 21-26, 2014

- You must own your animal 60 days prior to fair.

- It is recommended to purchase your animal late January/early February.

\section{Breeder Contact}

*If you are keeping your animal at the Agriculture Facility, you must purchase your animal through the Agriculture Department! If keeping your animal elsewhere, you may purchase your animal from a breeder of your choice. Below are some options:

- John Quinn (916) 719-8522

- Tom Miller (530) 934-0220 (Glenn)

- Small Town Genetics (209) 620-4106 (Denair

- Nick Gonzalez (530) 824-4292 (Richfield)
- Ottenwalter (530) 458-5700 (Colusa)

- Rex Mendoza (209) 988-1751

- Golden State Genetics (209) 277-2509

\section{Facility/Housing}

If you plan to raise your animal at home, make sure your pen meets the following requirements:

a) It is recommended to have $6 \mathrm{ft}^{2}$ covered space and the same amount of space outside for each animal.

b) Shade/shelter should be provided in the pen, with straw for bedding (during cold weather) at a location away from their feeder and water bucket so their sleeping area remains clean and dry.

c) Swine can be raised on dirt floor or concrete. If on concrete, be sure to have an exercise area to avoid lameness.

d) Adequate feeding and watering facilities must be provided.

e) The fencing and floor should be free of loose wires, nails, and boards.

f) Fencing is crucial! Enclosures should be at least 3 feet high. Strong wire fencing is best.

5. Advisor in Charge: Ms. Swenson, AC-1, iswenson@egusd.net, 916-686-0230 x4932 


\section{Market Lamb}

1. Sample Budget

\begin{tabular}{|l|c|}
\hline & \multicolumn{2}{|c|}{ Market Lamb } \\
\hline Cost of Animal & 300.00 \\
\hline Feed & \multicolumn{2}{|c|}{$\$ 00.00$} \\
\hline Show Supplies & 20.00 \\
\hline Entry Fees & 28.00 \\
\hline & \multicolumn{2}{|c|}{$\$$} \\
Veterinary Supplies & 10.00 \\
\hline Livestock Insurance & 20.00 \\
\hline & \multicolumn{2}{|c|}{$\$$} \\
Estimated Expenses & 678.00 \\
\hline & \multicolumn{2}{|c|}{$\$$} \\
\hline Sale of Animal & 617.50 \\
\hline & $130 \mathrm{lbs} @ \$ 4.75 / \mathrm{lb}$ \\
\hline & $\$$ \\
\hline Estimated Net Profit & $\mathbf{6 0 . 5 0}$ \\
\hline
\end{tabular}

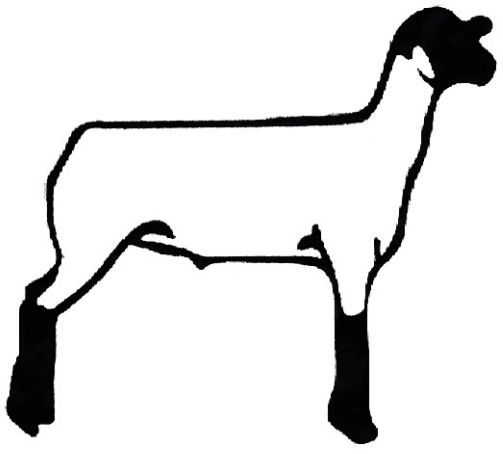

2. Ownership of Project

- Sacramento County Fair is May 21-26, 2014

- You must own your animal 60 days prior to fair.

- It is recommended to purchase your animal late January/early February.

\section{Breeder Contact}

*If you are keeping your animal at the Agriculture Facility, you must purchase your animal through the Agriculture Department! If keeping your animal elsewhere, you may purchase your animal from a breeder of your choice. Below are some options:

- Donnie Whitworth (530) 979-2006

- Ken \& Charlotte Mitchell (916) 761-5824

- Dana Van Liew (530) 752-0744 $\rightarrow$ Aggie Classic Club Lamb Sale in January 2014, UC Davis

- CSU Chico Sale: 2/8/14 @ 8am, University Farm Pavilion

4. Facility/Housing

If you plan to raise your animal at home, make sure your pen meets the following requirements:

a) Protection from cold drafts, rain and the sun. It should be large enough to allow your animal to exercise (About $15^{\prime} \times 20^{\prime}$ ).

b) Shade/shelter should be provided in the pen, with straw for bedding (during cold weather) at a location away from their feeder and water bucket so their sleeping area remains clean and dry.

c) Adequate feeding and watering facilities must be provided.

d) The fencing and floor should be free of loose wires, nails, and boards.

e) It should be sturdy enough to prevent the animal from getting out.

5. Advisor in Charge: Ms. Zallo, AC-1, izallo@egusd.net, 916-686-0230 x4934

6. Mandatory! $1^{\text {st }}$ Information Meeting on Thursday, January $30^{\text {th }}, 2014$ @ 3:30-4:15pm in AC-1. 


\section{Market Goat}

1. Sample Budget

\begin{tabular}{|c|c|c|}
\hline & \multicolumn{2}{|c|}{ Market Goat } \\
\hline Cost of Animal & $\$$ & 250.00 \\
\hline Feed & $\$$ & 175.00 \\
\hline Show Supplies & $\$$ & 20.00 \\
\hline Entry Fees & $\$$ & 28.00 \\
\hline Veterinary Supplies & $\$$ & 10.00 \\
\hline Livestock Insurance & $\$$ & 14.00 \\
\hline Estimated Expenses & $\$$ & 497.00 \\
\hline Sale of Animal & $\$$ & 500.00 \\
\hline & 100 & $\$ 5 / / \mathrm{b}$ \\
\hline Estimated Net Profit & $\mathbf{\$}$ & 3.00 \\
\hline
\end{tabular}

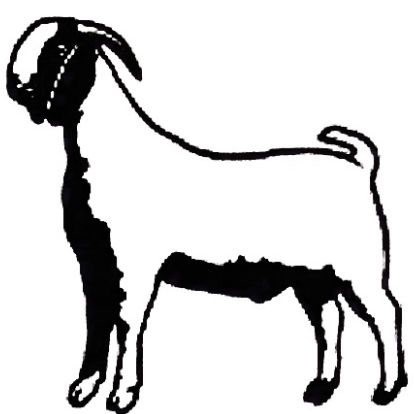

2. Ownership of Project

- Sacramento County Fair is May 21-26, 2014

- You must own your animal 60 days prior to fair.

- It is recommended to purchase your animal late January/early February.

3. Breeder Contact

*If you are keeping your animal at the Agriculture Facility, you must purchase your animal through the Agriculture Department! If keeping your animal elsewhere, you may purchase your animal from a breeder of your choice. Below are some options:

- Wilton Boer Goat Ranch (916) 687-8030

- Sundance Kids (209) 333-0543 (Stockton/Lodi)

- Cuneo Valley Ranch (209) 223-1570 (Jackson)

- Olive Knoll Ranch, Catherine Acly (209) 754-4580 (Angels Camp)

- Blevins Boer Goats (530) 695-3484 (Live Oak)

- Hazeltines (530) 681-0290

- HJ Boer Goats (209) 614-5664 (Oakdale)

\section{Facility/Housing}

If you plan to raise your animal at home, make sure your pen meets the following requirements:

a) Protection from cold drafts, rain and the sun. It should be large enough to allow your animal to exercise (About $15^{\prime} \times 20^{\prime}$ ).

b) Shade/shelter should be provided in the pen, with straw for bedding (during cold weather) at a location away from their feeder and water bucket so their sleeping area remains clean and dry.

c) Adequate feeding and watering facilities must be provided.

d) The fencing and floor should be free of loose wires, nails, and boards.

e) It should be sturdy enough to prevent the animal from getting out.

5. Advisor in Charge: Ms. Zallo, AC-1, izallo@egusd.net, 916-686-0230 x4934 


\section{Market Steer}

1. Sample Budget

\begin{tabular}{|l|l|}
\hline & Market Steer \\
\hline & $\$$ \\
Cost of Animal & $1,700.00$ \\
\hline Feed & $\$$ \\
\hline & $\$$ \\
Show Supplies & 100.00 \\
\hline & $\$$ \\
Entry Fees & 40.00 \\
\hline & $\$$ \\
Veterinary Supplies & 50.00 \\
\hline & $\$$ \\
Livestock Insurance & 75.00 \\
\hline Estimated Expenses & $\$$ \\
\hline & $2,790.00$ \\
\hline Sale of Animal & $\$$ \\
\hline & $12501 \mathrm{lbs} @ \$ 1.957 .50$ \\
\hline & $\$$ \\
\hline Estimated Net Profit & $\mathbf{- 3 5 2 . 5 0}$ \\
\hline
\end{tabular}

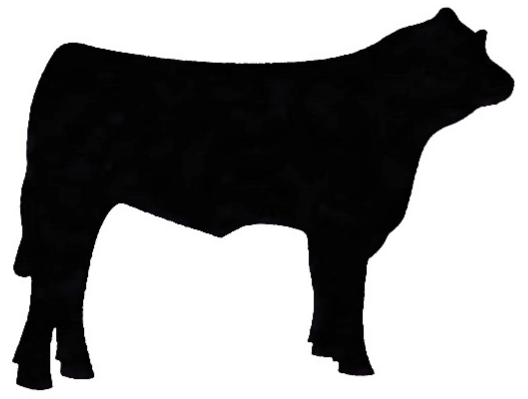

\section{Ownership of Project}

- Sacramento County Fair is May 20-26, 2014

- You must own your animal 120 days prior to fair.

- It is recommended to purchase your animal late September/early October.

\section{Breeder Contact}

Please see specie advisor

\section{Facility/Housing}

Have your pen prepared before receiving your beef project. The pen should meet the following requirements:

a) It should be large enough to allow your animal to exercise. (Approximately $15^{\prime} \times 20^{\prime}$ is adequate.)

b) Shade/shelter should be provided in the pen.

c) Adequate feeding and watering facilities must be provided.

d) The fencing should be free of loose wires, loose boards, protruding nails, etc. The floor should also be free of boards, wire, nails, etc.

e) It should be sturdy enough to prevent the animal from getting out and also provide some place to tie the animal when working with it.

5. Advisor in Charge: Ms. Forster, AC-2, jorster@egusd.net 


\section{Market Turkey \\ 1. Sample Budget}

\begin{tabular}{|l|c|}
\hline & Market Turkey \\
\hline Cost of Animal & $\$$ \\
\hline & 10.00 \\
\hline Feed & $\$$ \\
& 30.00 \\
\hline Show Supplies & $\$$ \\
\hline & 2.00 \\
\hline Entry Fees & $\$$ \\
\hline Veterinary Supplies & 6.00 \\
\hline & $\$$ \\
\hline Estimated Expenses & $\$$ \\
\hline & 48.00 \\
\hline Sale of Animal & $\$$ \\
\hline & \\
\hline & $\$$ \\
\hline Estimated Net Profit & $\mathbf{5 2 . 0 0}$ \\
\hline
\end{tabular}

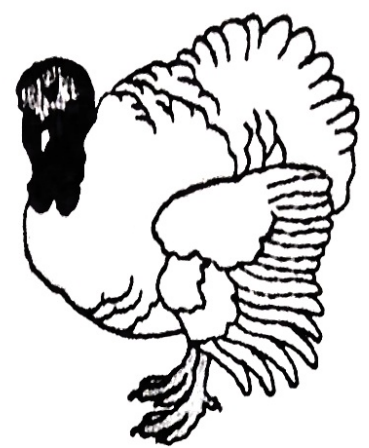

2. Ownership of Project

- Sacramento County Fair is May 21-26, 2014

- You must own your animal 30 days prior to fair.

- It is recommended to purchase your animal late January/early February.

3. Breeder Contact

*If you are keeping your animal at the Agriculture Facility, you must purchase your animal through the Agriculture Department!

- Jenna Swenson, AC-1 (jswenson@egusd.net); 916-686-0230 x4932

\section{Facility/Housing}

If you plan to raise your animal at home, make sure your space meets the following requirements:

- Initially, provide approximately 2 square feet per poult and increase space over time. As bird ages, you will need to provide a cage for the chicken or a pen for the turkeys.

- You will need a brooding lamp with infrared bulb. Hang it 18 inches above surface of the bedding (shavings). Allow enough space for poults to move to/away from heat source as they please.

- You will need a feeder and a waterer.

- Ventilation must be provided for bird comfort, but avoid drafty areas especially at the beginning.

5. Advisor in Charge: Ms. Swenson, AC-1, jswenson@egusd.net, 916-686-0230 x4932 


\section{-Market Rabbit}

1. Sample Budget

\begin{tabular}{|c|c|c|}
\hline & \multicolumn{2}{|c|}{ Single Fryer (1) } \\
\hline Cost of Animal & $\$$ & 25.00 \\
\hline Feed & $\$$ & 25.00 \\
\hline Show Supplies & $\$$ & - \\
\hline Entry Fees & $\$$ & 6.00 \\
\hline Veterinary Supplies & & \\
\hline Estimated Expenses & $\$$ & 56.00 \\
\hline Sale of Animal & $\$$ & 100.00 \\
\hline Estimated Net Profit & $\$$ & 44.00 \\
\hline
\end{tabular}

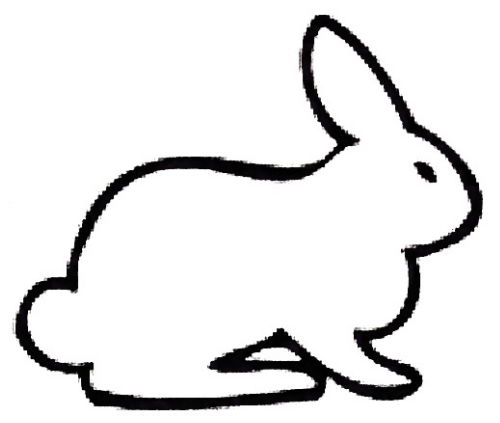

\section{Ownership of Project}

- Sacramento County Fair is May 21-26, 2014

- You must own your animal 30 days prior to fair.

- It is recommended to purchase your animal mid to late April.

\section{Breeder Contact}

*If you are keeping your animal at the Agriculture Facility, you must purchase your animal through the Agriculture Department!

\section{Facility/Housing}

If you plan to raise your animal at home, make sure your space meets the following requirements:

- The cage should be about 4 times larger than your rabbit. It should be large enough for a litter box area (unless it has a wire bottom, in which case you would have a pan underneath to collect urine, feces, falling hay, bedding \& food). You should provide a solid area of flooring with a board over the wire floor for your rabbit's comfort.

- You will need a feeder and waterer.

5. Advisor in Charge: Ms. Forster, AC-2, iforster@egusd.net, 916-686-0230 x4933 
ne Point Award System was developed in order to recognize and award those members who are most active in various FFA activities throughout the year. The top ten individuals for each grade level will receive a certificate and recognition at our awards banquet in May. These top forty members will also be invited to participate in a Point Award trip sponsored by the Pleasant Grove FFA. In 2013, the Point Award members went to 6 Flags Amusement Park in Vallejo.

The Point Award System is an evaluation resource used by the Advisors towards selecting each year's Star Chapter Greenhand and Star Chapter Farmer award recipient as well as the Outstanding Junior and Outstanding Senior Awards. 


\section{ELK GROVE-PLEASANT GROVE HIGH SCHOOL FFA CHAP'TER CONSTITUTION}

\section{Article I: Name \& Purpose}

Section A: The name of this organization shall be the "Pleasant Grove Chapter of the FFA", members are hereinafter referred to as "FFA", and the letters "FFA", may be used to designate the Chapter, its activities, or members thereof.

Section $B$ : The purposes for which this chapter is formed are as follows:

1. To develop competent and aggressive agricultural leadership.

2. To create and nurture a love of agricultural life.

3. To strengthen the confidence of students of vocational agriculture in themselves and their work.

4. To create more interest in the intelligent choice of agricultural occupations.

5. To encourage members in the development of individual supervised agricultural experience programs and establishment in agricultural careers.

6. To encourage members in wise management of economic, environmental and human resources of the community.

7. To develop interpersonal skills in teamwork, communication, human relations and social interactions.

8. To build character and promote citizenship, volunteerism and patriotism.

9. Promote cooperation and cooperative attitudes among all people.

10. To promote healthy lifestyles.

11. To encourage excellence in scholarship.

\section{rticle II: Organization}

Section A: The Pleasant Grove Chapter of FFA is a chartered local unit of the California Association of FFA which is chartered by the National FFA Organization.

Section B: This chapter accepts in full the provisions in the constitution and bylaws of the California Association of FFA as well as those of the National FFA Organization.

\section{Article III: Membership}

Section A: Membership in this chapter shall be of three kinds: (1) Active; (2) Alumni and (3) Honorary, as defined by the National FFA Constitution.

Section B: The regular activities of this chapter shall be carried on by the active membership.

Section $C$ : Honorary membership in this chapter shall be limited to the Honorary Chapter FFA Degree.

Section D: Active members in good standing may vote on all business brought before the chapter. An active member shall be considered in good standing when:

1. While in school, be enrolled in at least one agricultural education course during the school year and/or follow a planned course of study. Either course must include a supervised agricultural experience program, the objective of which is preparation for an agricultural career.

2. Show an interest in the affairs of the organization by attending meetings, striving for degrees of membership, and participating in other organized activities of the chapter.

3. Pay all current state and national dues by the date determined by the chapter. 
4. Display conduct consistent with the ideals and purposes of the National FFA Organization.

Section E: Names of applicants for membership shall be filed with the membership committee.

Section F: Any student enrolled in a vocational Agriculture class is automatically a member of the FFA.

\section{Article IV: Emblems}

Section A: The emblem of the FFA shall be the emblem for the chapter.

Section B: Emblems used by members shall be designated by the National FFA Organization.

\section{Article V: Membership Degrees and Privileges}

Section A: There shall be four degrees of active membership in this chapter based on individual achievement. These degrees are (1) Greenhand FFA Degree, (2) Chapter FFA Degree, (3) State FFA Degree, and (4) American FFA Degree.

All "Greenhands" are entitled to wear the regulation bronze emblem pin. All members holding the Chapter FFA Degree are entitled to wear the regulation silver emblem pin. All members holding the State FFA Degree are entitled to wear the regulation gold emblem charm. All members holding the American FFA Degree are entitled to wear the regulation gold emblem

key.

Section B: Greenhand FFA Degree. Minimum qualifications for election:

1. Be enrolled in agricultural education and have satisfactory plans for a supervised agricultural experience program.

2. Learn and explain the meaning of the FFA Creed, Motto, and Salute.

3. Describe and explain the meaning of the FFA emblem and colors.

4. Demonstrate knowledge of the FFA Code of Ethics and the proper use of the FFA jacket.

5. Demonstrate knowledge of the history of the organization, the chapter constitution and bylaws, and the chapter Program of Activities.

6. Personally own or have access to the Official FFA Manual and the FFA Student Handbook.

7. Submit a written application for the Greenhand FFA Degree.

Section C: Chapter FFA Degree. Minimum qualifications for election:

1. Must have received the Greenhand FFA Degree.

2. Must have satisfactorily completed at least one year of instruction in vocational agriculture, have in operation an approved supervised agricultural experience program, and be enrolled in an agricultural education course.

3. Be familiar with the purposes and programs of activities of the State Association and National Organization.

4. Must have earned and productively invested at least $\$ 150$ by the member's own efforts or worked at least forty-five hours in excess of scheduled class time, or a combination thereof, and have developed plans for continued growth and improvement in a supervised agricultural experience program.

5. Be familiar with the provisions of the constitution of the local chapter.

6. Must have effectively led a group discussion for 15 minutes.

7. Be familiar with parliamentary procedure.

8. Must submit a written application for the Chapter FFA Degree.

Section D: State FFA Degree. Minimum qualifications for selection: 
1. Qualifications for the State FFA Degree are those set forth in the constitution of the State FFA Association.

?ction E: American FFA Degree. Minimum qualifications for selection:

1. Qualifications for the American FFA Degree are those set forth in the constitution of the National FFA Organization.

Section F: Special committees shall review the qualifications of members and make recommendations to the chapter concerning degree advancement.

\section{$\underline{\text { ARTICLE VI: State and Chapter Officers }}$}

Section A: The offices of the FFA chapter shall be: president, vice president, secretary, treasurer, reporter and sentinel. Other officers may be elected as deemed appropriate by the local advisor and/or governing body. The teacher(s) of agricultural education shall be the FFA advisor(s). Officers shall perform the usual duties of their respective offices.

Section B: Concerning the election of chapter officers, all potential officers must go through a screening committee which will consist of past year's officers, school administrator(s), teacher(s), and the advisors. The committee will assign two individuals per office who they feel is qualified to be a FFA officer. Candidates will prepare a speech (no longer than 2 minutes) to address to the FFA membership. The chapter members will then vote on one person per FFA office to represent them.

Section $C$ : The officers of the chapter together with the chairpersons in charge of the major sections of the annual program of activities shall constitute the Chapter Executive Committee. The Executive Committee shall zve full power to act as necessary for the chapter in accordance with actions taken at chapter meetings and various regulations or bylaws adopted from time to time.

Section D: Chapter officers must hold the degree of Chapter Farmer, except during the first year the chapter is organized.

Section E: Honorary members shall not vote nor shall they hold any office in the chapter except that of advisor.

Section F: Chapter officers must maintain a "C" (2.0) average of all classes started each semester or undergo consequences stated in officer contract.

Section $G$ : Officers will attend the following mandatory activities: officer training/retreat; chapter meetings; officer meetings; and other activities deemed necessary by the president and the advisor.

Section $H$ : An officer may be removed from the officer team by a majority vote of the officer team or by the advisor for not meeting the requirements as laid out in the officer contract or in Section $G$ of this constitution.

Section I: Duties of the Chapter Officers.

\section{- President:}

1. Preside over and conduct meetings according to accepted rules of parliamentary procedure.

2. Call special meetings.

3. Keep members on the subject and within the time limit.

4. Appoint committees and serve on them as an ex-officio (non-voting) member.

5. Coordinate the activities of the chapter and evaluate the progress of each division of the Program of Activities. 
6. Represent the chapter in public relations and official functions.

- Vice President:

1. Assume all duties of the president, if necessary.

2. Develop the Program of Activities and serve as an ex-officio (non-voting) member of the POA

3. Coordinate all committee work.

4. Work closely with the president and advisor to assess progress toward meeting chapter goals.

5. Establish and maintain a chapter resource file.

- Secretary:

1. Prepare and post the agenda for each chapter meeting.

2. Prepare and present the minutes of each chapter meeting.

3. Prepare and place all committee reports in the designated area in the Official FFA Chapter

4. Be responsible for chapter correspondence.

5. Count and record rising vote when taken.

6. Send out and post notices.

7. Keep the permanent record of the chapter.

8. Maintain member attendance and activity records and issue membership cards.

9. Keep the Program of Activities wall chart up-to-date.

10. Cooperate with the treasurer in keeping an accurate membership roll and issue member cards.

11. Have on hand for each meeting:

- Official FFA Chapter Secretary's Book, including minutes of the previous meeting.

- Lists of committees and committee reports.

- Copy of the Program of Activities, including all standing and special committees.

- Official FFA Manual and Student Handbook.

- Copies of the chapter constitution and bylaws.

- Treasurer

1. Receive, record, and deposit FFA funds and issue receipts.

2. Assist in preparing an annual budget of estimated receipts and expenditures.

3. Present monthly treasurer's reports at chapter meetings.

4. Pay out chapter funds as authorized.

5. Collect dues and special assessments.

6. Maintain a neat and accurate Official FFA Chapter Treasurer's Book.

7. Prepare and submit the membership roster and dues to the National FFA Organization in cooperation with the secretary.

8. Serve as chairperson of the earnings and savings committee.

\section{- Reporter}

1. Plan public information programs with local radio, television, newspaper, and service clubs and make use of other opportunities to tell the FFA story.

2. Release news and information to local and regional news media.

3. Publish a chapter newsletter.

4. Assist in maintaining a chapter bulletin board.

5. Serve as chapter historian. Prepare and maintain a chapter scrapbook.

6. Send local stories to area, district, and state reporters. 7. Send articles and photographs to FFA New Horizons and other national and/or regional
publications. 
8. Work with local media on radio and television appearances and FFA news.

9. Serve as the chapter photographer.

- $\underline{\text { Sentinel }}$

1. Assist the president in maintaining order.

2. Keep the meeting room, chapter equipment, and supplies in proper condition.

3. Welcome guests and visitors.

4. Keep the meeting room comfortable.

5. Take charge of candidates for degree ceremonies.

6. Assist with special features and refreshments.

- $\underline{\text { Advisor }}$

1. Assume the initiative for getting a chapter established in the school.

2. Become familiar with the history, principles, constitutional provisions, ceremonies, typical activities, parliamentary procedures, and other essentials of the organization.

3. Instruct newly elected officers in their duties and provide leadership training for all members.

4. See that programs are adequately financed and chapter funds properly protected.

5. See that chapter meetings are held regularly throughout the year and conducted in a business-like manner.

6. Assist with plans for securing an efficient set of officers.

7. Supervise chapter activities year-round.

8. Inform prospective students and parents about the FFA.

9. Instruct students in leadership and personal development.

10. Build school and community support for the program.

11. Encourage involvement of all chapter members in activities.

12. Prepare students for involvement in contests and awards programs.

\section{ARTICLE VII: Meetings}

Section A: Regular chapter meetings shall be held once per month during the school year. Special meetings may be called at any time including summer. Meetings will be held the first Wednesday of each month and is subject to change by the Executive Committee or advisor.

Section B: Standard meeting paraphernalia shall be used at each meeting. All regular meetings shall open and close with the official ceremony. Parliamentary procedure shall be used in transacting all business at each meeting.

Section C: Delegates for the State FFA Convention will be chosen by the Executive Committee and advisor. Criteria will be based on essays written by applicants and participation in FFA activities.

Section D: A majority of the active members listed on the secretary's membership roll shall constitute a quorum, and a quorum must be present at any meeting at which business is transacted, or a vote taken committing the chapter to any proposal or action.

Section E: Officers will only be allowed to miss a meeting, with permission of the president and approved by the advisor.

Section F: Conduct shall be in orderly fashion. All members attending the meeting must be properly dressed and meeting will be called to order on time. There will be a warning given for talking out of order or being late at the first offense. If the member does not then come to order on the second offense, then the sentinel will escort the member out of the meeting, and no credit will be given. 


\section{$\underline{\text { ARTICLE VII: Dues }}$}

'ction A: Local dues in this chapter shall be fixed annually by a majority vote of the active members.

Section $B$ : Full local, state and national dues shall be paid by all active members.

Section C: No member shall be considered as active and in good standing unless he/she pays full local, state and national FFA dues.

\section{ARTICLE VIII: Amendments}

Section A: This constitution may be amended or changed at any regular chapter meeting by a two-thirds vote of the active members present providing it is not in conflict with the state association constitution or that of the National FFA Organization.

Section B: Bylaws may be adopted to fit the needs of the chapter at any regular chapter meeting by a two-thirds vote of the active members present providing such bylaws conflict in no way with the constitution and bylaws of either the state association or the national organization.

Adopted December 7, 2005

Modified January 10, 2007 


\section{Chapter ByLaws}

The officers of the Pleasant Grove FFA serve as the core of the chapter. They must be dedicated and efficient in their duties in order to be effective. Consequently, the following by-laws have been established and accepted.

A. All officers will attend the following events in full uniform as representatives of Pleasant Grove FFA:

- All chapter meetings

- Chapter Officer Leadership Conference

- Participate in Opening/Closing Ceremonies Contest

- Annual Awards Banquets

- Officer Retreat (no uniform)

Failure to attend the above mentioned activities, without prior notification and approval by the president and advisor, will result in the following consequences:

- First offense: warning;

- Second offense: removal from the officer team and replaced.

B. All officers will meet on a weekly basis for executive meetings, or as needed. If an officer is late without prior notification and approval by the FFA president and advisor, then the officer will have the following consequence:

- 1-5 minutes late: the officer receives a warning;

- 5-15 minutes late: the officer receives 30 minutes of community service;

- After 15 minutes late: it will be considered an absence.

If an officer misses an executive meeting, chapter function or leadership activity and does not inform the chapter advisor at least one week prior to the event that he/she will not be able to attend, then the officer will have the following consequence:

- First offense: community service for 45 minutes;

- Second offense: community service for 1 hour;

- Third offense: removal from the officer team.

C. All officers shall maintain a minimum GPA of 2.0. Any officer who earns less than a $2.0 \mathrm{GPA}$ will be placed on probation for the following academic quarter. Upon completion of the academic quarter, if said officer raises his/her GPA to the required level, probation will be lifted. If said officer fails to raise his/her GPA to the appropriate level, he/she will be relieved of his/her officer duties for the Pleasant Grove FFA chapter. Elections shall be held in order to fill the resulting vacancy.

D. Any officer acting in a manner which is not consistent with the high ideals and expectations of the local, state, and national FFA organizations as regulations of said organization, then a special meeting will be scheduled with all officers and advisor(s) to discuss the situation and set the consequence as either:

- Probation for one academic quarter -or -

- Removal from team 
If placed on probation and second offense occurs, said officer will be removed from the team.

Mection II Responsibilities of Members

A. It is the members' responsibilities to attend the required FFA events as stated in their class syllabus.

Adopted December 7, 2005

Modified October 4, 2006; November 2, 2006; July 16, 2008; May 1, 2010 